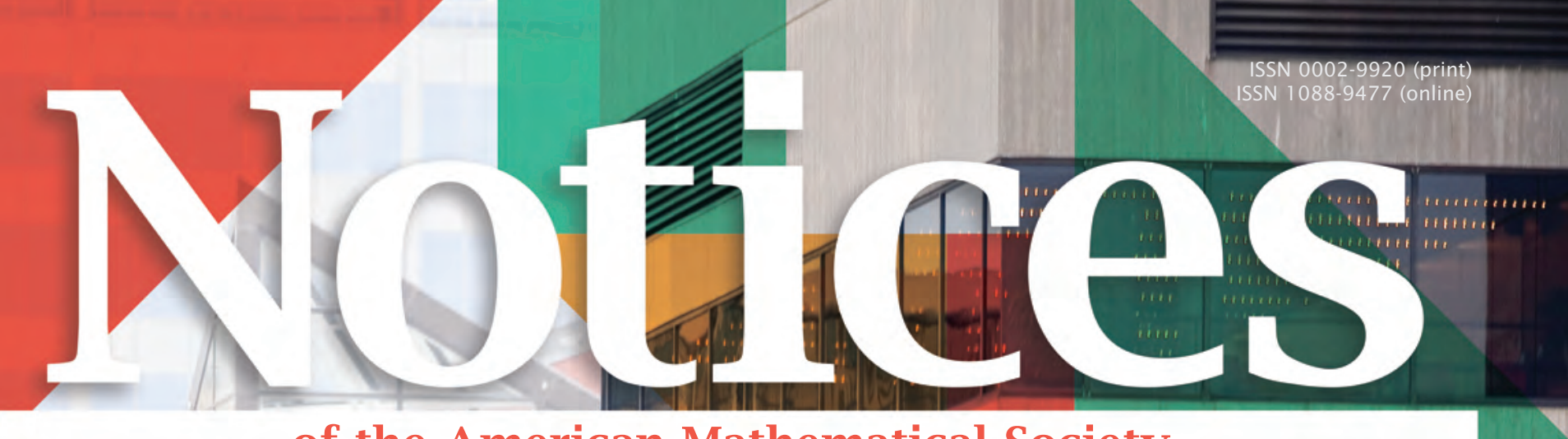

\title{
of the American Mathematical Society
}

\section{October 2018}

The On-Line Encyclopedia of Integer Sequences

page 1062

AMS Fall Western Sectional Sampler

\section{page 1076}

Statistics on

Women Mathematicians

page 1096

Baltimore Meeting-JMM 2019

page 1163

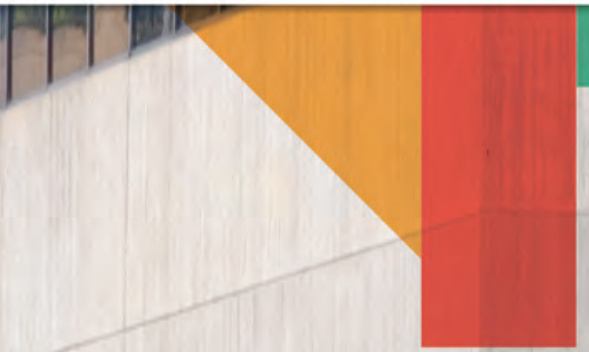

Volume 65, Number 9
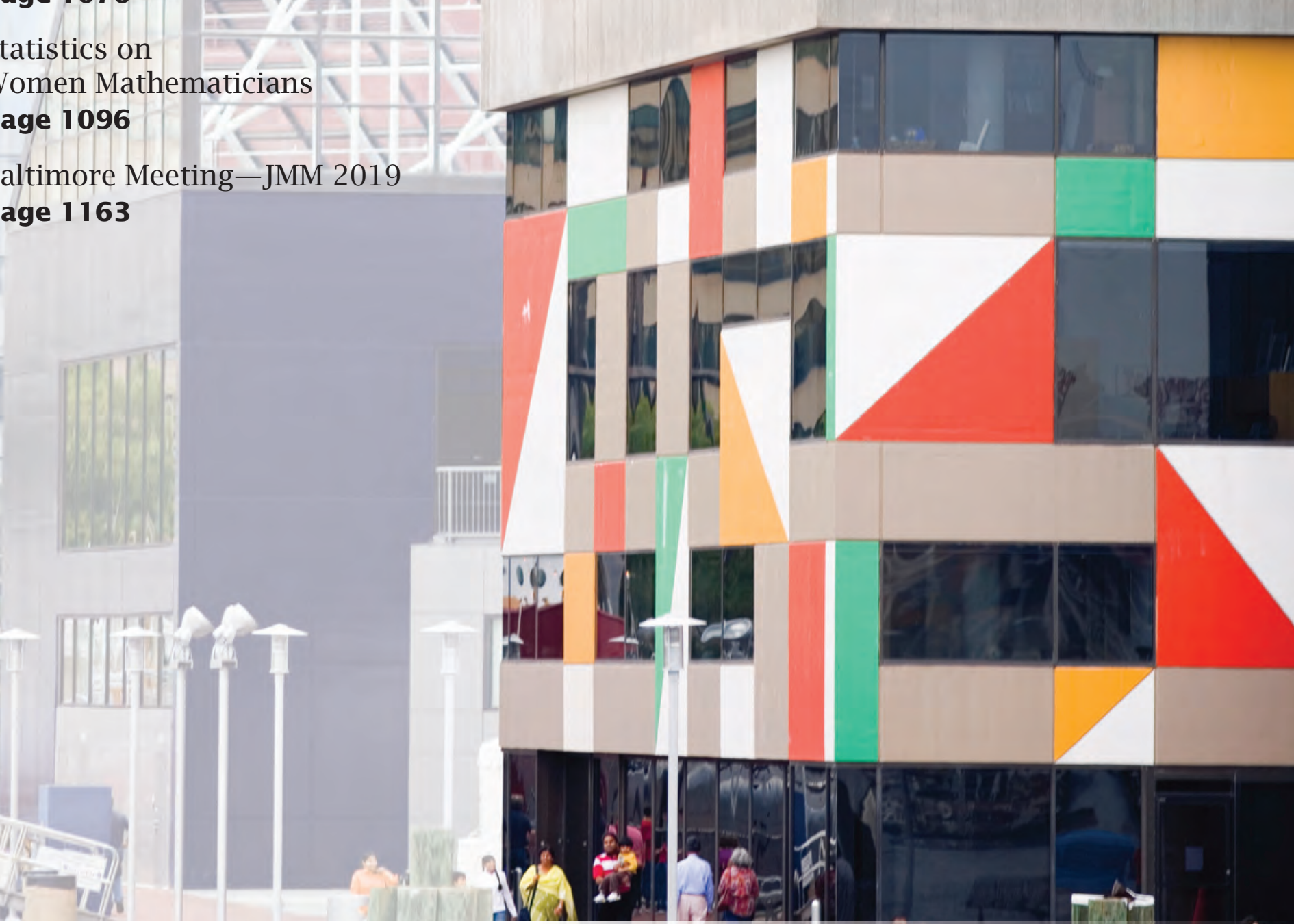

AMMS
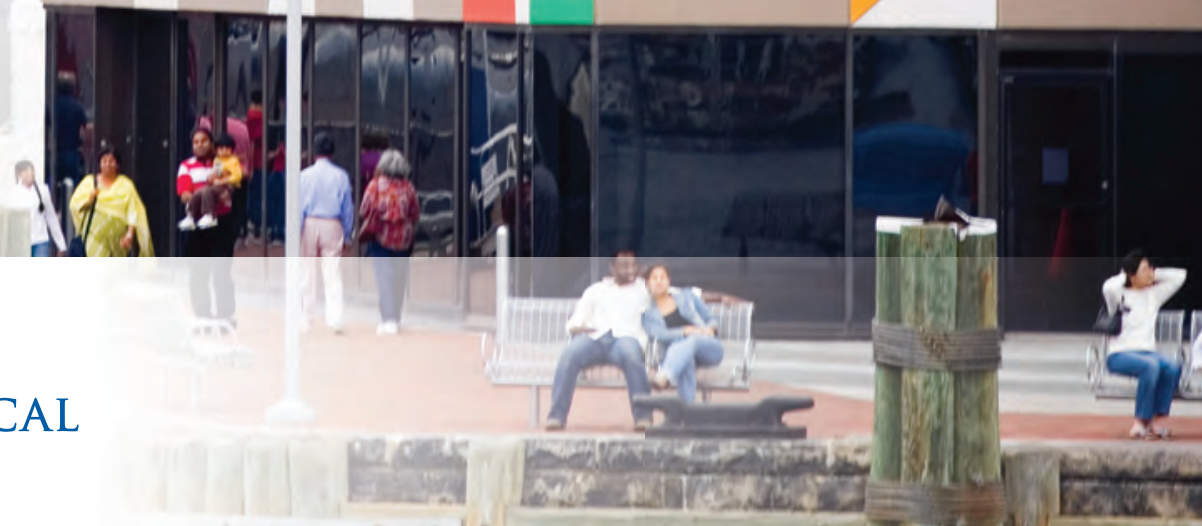


\section{Attend the AMS 2019 JMM Social at the Maryland Science Center}

Guests will enjoy the evening at this Baltimore destination with access to exhibits and hands-on displays. Join us for live music, various food stations, interactive activities, and the opportunity to connect with enthusiastic members of the mathematics community. It is a great chance to wish your colleagues well "until next time"!

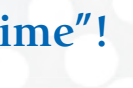



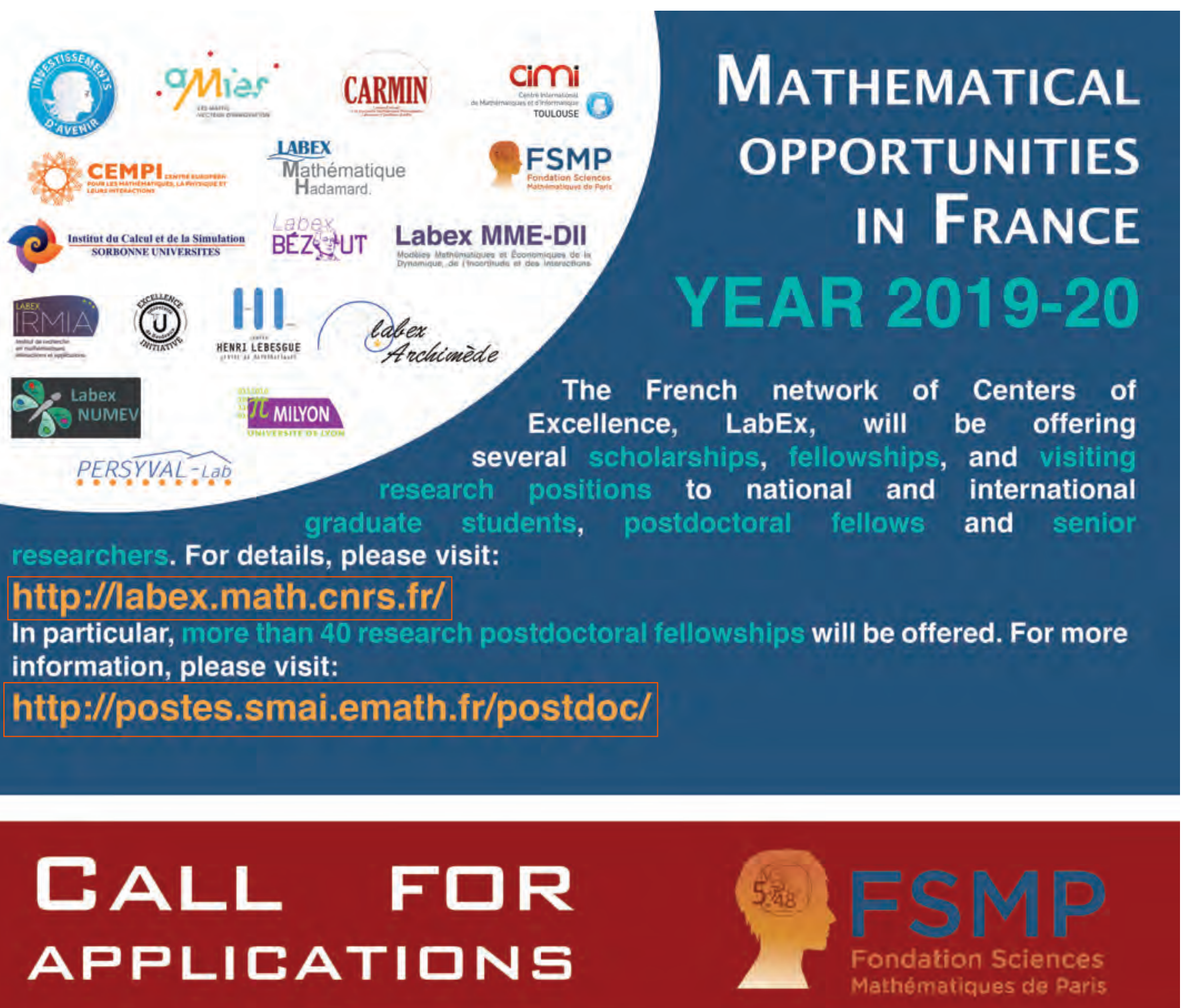

\section{PARIS = ACADEMIC YEAR 2D 19-2020}

The Fondation Sciences Mathématiques de Paris (FSMP) is accepting applications for its various programs in support of research and training in mathematical sciences at anyone of its affiliated laboratories in the Paris area. Senior and junior scientists in mathematics and fundamental computer science as well as graduate students are welcome to apply to anyone of the following programs that correspond to their career situation.

\begin{tabular}{l|c|c|r}
$\begin{array}{l}\text { Research chairs } \\
\text { of excellence }\end{array}$ & $\begin{array}{c}\text { Postdoctoral } \\
\text { positions }\end{array}$ & $\begin{array}{r}\text { Ph.D } \\
\text { allowances }\end{array}$ & $\begin{array}{r}\text { Master } \\
\text { scholarships }\end{array}$ \\
2 to 5 laureates/year & 25 positions/year & 30 thesis/year & 35 scholarships/year \\
4 to 12 months in Paris & 1 or 2 years in Paris & 3 years in Paris & or 2 years in Paris
\end{tabular}
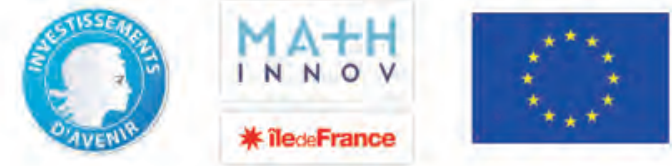

Online applications starting on October 1, 2018 www.sciencesmaths-paris.fr Email: contact@fsmp.fr 


\section{CIB}

\section{Call for proposals July 2020 - June 2022}

The Bernoulli Center (CIB) in Lausanne invites you to propose a one-semester programme in any branch of the mathematical sciences.

Such a programme will benefit from the resources and funding of CIB, allowing for long-term and short-term visitors, conferences, seminars, workshops, lecture series or summer schools.

You, together with one or more colleagues, could be the scientific organiser of such a semester and rely on the dedicated staff of CIB to prepare and run the programme. We invite you to submit a 2 to 4 page letter of intent by November 25, 2018. This submission should outline the programme and indicate already the key participants that are envisioned. Write to the CIB director Nicolas Monod at cib.director@epfl.ch.

Past programmes and general information can be viewed on http://cib.epfl.ch.

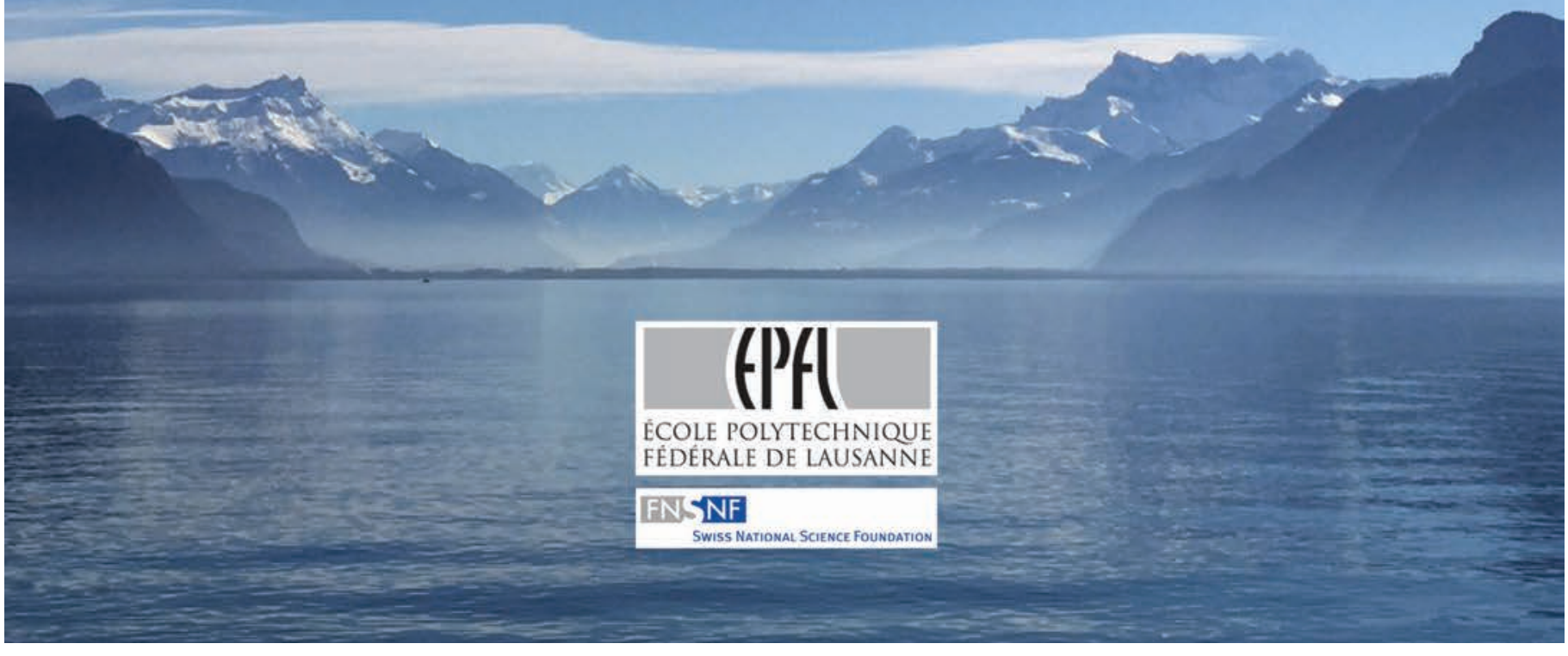




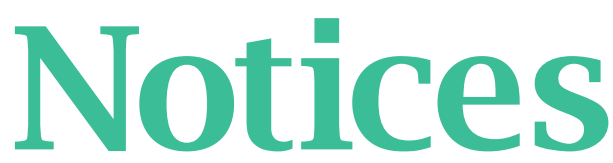

of the American Mathematical Society
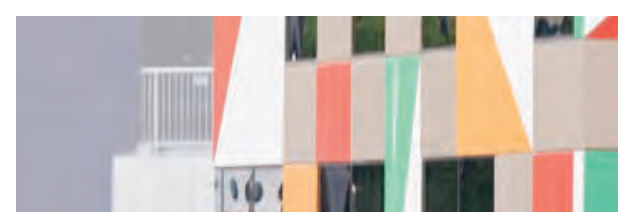

\section{FEATURED}

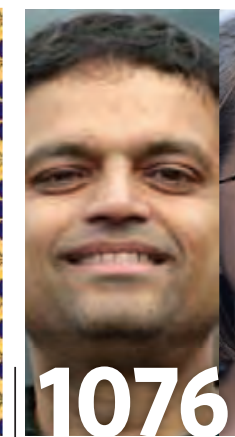

AMS Fall Western Sectional Sampler

Srikanth B. Iyengar, Sarah

Witherspoon, and Abdul-Aziz Yakubu

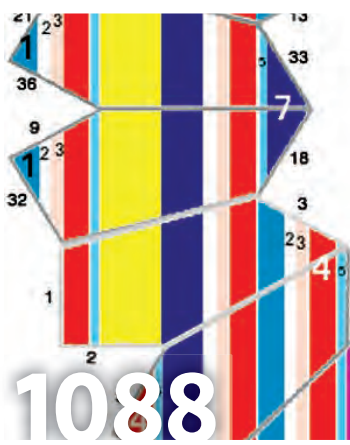

$(2)=(3)=(5)$

$(6)=(1 ;$

(7) $=(9$

(8) $=$$$
4
$$

8

8 10 $9.13 / 234$

\section{Geometry Labs United:}

An Invitation

Jayadev Athreya, David Dumas,

William Goldman, Sergey Grigorian,

Rosemary Guzman, Philipp Hieronymi,

Sean Lawton, Anton Lukyanenko,

Jeremy Tyson, and Aaron Wilson

"This is wonderful.... It will be read by thousands for many years to come," wrote one editor upon seeing Neil Sloane's article The On-Line Encyclopedia of Integer Sequences. The sequences and related unsolved problems are fascinating. This article alone will so stimulate your hunger for math that you will want to register immediately for the January 2019 Joint Mathematics Meetings in Baltimore. Meanwhile, you can enjoy the lecture samplers herein for this month's Western Sectional at San Francisco State. We've also got an interview with Juan Meza, the new director for the Division of Mathematical Sciences at the National Science Foundation. -Frank Morgan, Editor-in-Chief

\section{JMM 2019:}

1115 Mathematical Sciences Employment Center in Baltimore

1131 AMS Short Course in Baltimore

1163 Baltimore Meeting Special Section

1165 Program Announcements

1200 Baltimore City Map

1201 Program Timetable at a Glance

1215 Baltimore Meeting Registration Forms

\section{ALSO IN THIS ISSUE}

1098 Book Review: Reverse Mathematics Carl Mummert

1104 Interview with NSF DMS Director Juan Meza Robert L. Bryant

\section{GRADUATE STUDENT SECTION}

1084. Ruth Haas Interview Alexander Diaz-Lopez

1086 WHAT IS...a Rectifiable Set?

\section{FROM THE AMS SECRETARY}

1096 Statistics on Women Mathematicians, Compiled by the AMS

1108 Report of the AMS Treasurer-2017

1127 Call for Suggestions for 2019 Elections 


\section{IN EVERY ISSUE}

\section{The Back Page}

1118 Mathematics People

\section{Mathematics Opportunities}

1128 Inside the AMS

\section{Classified Advertising}

1140 New Publications Offered by the AMS

1149 Meetings and Conferences of the AMS

Cover: National Aquarium, Inner Harbor, Baltimore, Maryland, USA. Credit: Glowimages. Courtesy of Getty Images.

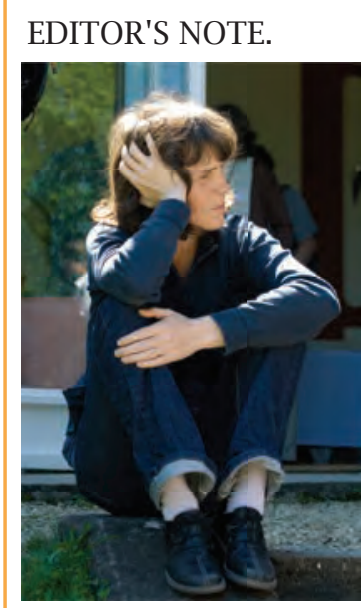

Jean-Pierre Bourguignon kindly sent us this color version of a photo we ran in "Ad Honorem Claire Voisin" in the April 2018 Notices. The photo was taken at the conference at IHÉS for his 60th birthday by Jean-François Dars.
AMS / MAA Press

NEVER A DULL MOMENT HASSLER WHITNEY, MATHEMATICS PIONEER

Keith Kendig, Cleveland State University, $\mathrm{OH}$

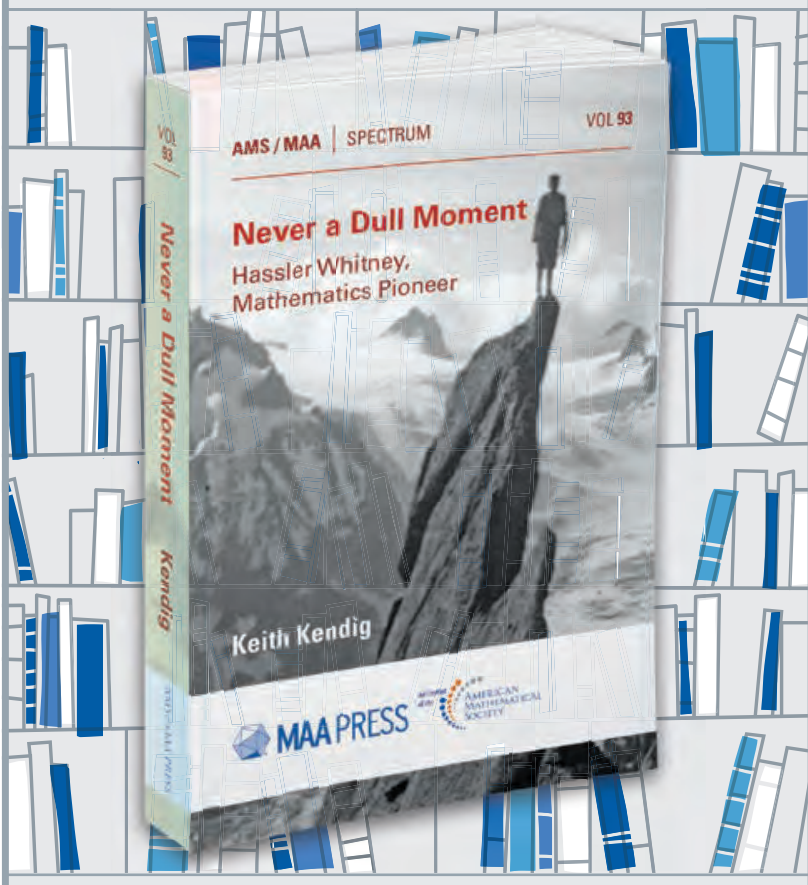

This biography is a revealing portrait of a fascinating personality and a titan of twentieth-century mathematics, Hassler Whitney. Whitney is probably best known for introducing the grandfather of today's innumerable embedding theoremshis strong embedding theorem stating that any smooth manifold can be smoothly embedded in a Euclidean space of twice the manifold's dimension. This in turn led to several standard techniques used every day in algebraic topology. Whitney also established the fundamentals of graph theory, the four-color problem, matroids, extending smooth functions, and singularities of smooth functions. He almost never used complicated technical machinery, so most of his work is accessible to a general reader with a modest mathematical background.

Spectrum, Volume 93; 2018; approximately 381 pages; Softcover ISBN: 978-1-4704-4828-8; List US\$60; AMS Individual member US $\$ 45$; MAA members US\$45; Order code SPEC/93

\section{AVAILABLE IN EBOOK FORMAT}

This title is now available for pre-order at bookstore.ams.org/spec-93

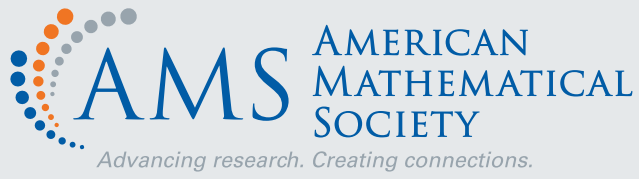




\section{EDITOR-IN-CHIEF \\ Frank Morgan}

\section{ASSOCIATE EDITORS}

Benjamin Braun,

Alexander Diaz-Lopez, Thomas Garrity, Joel Hass,

Stephen Kennedy, Florian Luca, Steven J. Miller, Harriet Pollatsek, Carla Savage (ex officio),

Cesar E. Silva, Christina Sormani, Daniel J. Velleman

CONSULTANTS

John Baez, Hélène Barcelo, Ricardo Cortez, Jerry Folland, Tara Holm, Kenneth A. Ribet

\section{ASSISTANT to the EDITOR-IN-CHIEF Sophia D. Merow \\ MANAGING EDITOR Rachel L. Rossi \\ ADVERTISING COORDINATOR \\ Anne Newcomb \\ REPRINT PERMISSIONS \\ Erin M. Buck \\ CONTRIBUTOR \\ Elaine Kehoe}

COMPOSITION, DESIGN, and EDITING

Brian Bartling, John Brady, Anna Hattoy, Lori Nero,

Karen Ouellette, Rebecca Rivard, Courtney Rose,

Deborah Smith, Mike Southern, Peter Sykes

Supported by the AMS membership, most of this publication, including the opportunity to post comments, is freely available electronically through the AMS website, the Society's resource for delivering electronic products and services. Use the URL www.ams.org/notices/to access the Notices on the website. The online version of the Notices is the version of record, so it may occasionally differ slightly from the print version.

The print version is a privilege of Membership. Graduate students at member institutions can opt to receive the print magazine by updating their individual member profiles at http://www.ams. org/member-directory. For questions regarding updating your profile, please call 800-321-4267.

For back issues see www.ams.org/backvols. Note: Single issues of the Notices are not available after one calendar year.

\section{CONTACTING THE NOTICES}

\section{SUBSCRIPTION INFORMATION}

Subscription prices for Volume 65 (2018) are US\$662 list; US\$529.60 institutional member; US\$397.20 individual member; US $\$ 595.80$ corporate member. (The subscription price for members is included in the annual dues.) A late charge of $10 \%$ of the subscription price will be imposed upon orders received from non-members after January 1 of the subscription year. Add for postage: Surface delivery outside the United States and IndiaUS\$27; in India-US\$40; expedited delivery to destinations in North America-US\$35; elsewhere-US\$120. Subscriptions and orders for AMS publications should be addressed to the American Mathematical Society, PO Box 845904, Boston, MA 02284-5904 USA. All orders must be prepaid.

\section{ADVERTISING}

Notices publishes situations wanted and classified advertising, and display advertising for publishers and academic or scientific organizations. Advertising requests, materials, and/or questions should be sent to:

\section{classads@ams.org (classified ads) notices-ads@ams.org (display ads) \\ PERMISSIONS}

All requests to reprint Notices articles should be sent to: reprint-permission@ams.org

\section{SUBMISSIONS}

The editor-in-chief should be contacted about articles for consideration after potential authors have reviewed the "For Authors" page at www.ams.org/publications/journals/notices/ noticesauthors.

The managing editor should be contacted for additions to our news sections and for any questions or corrections. Contact the managing editor at: notices@ams.org

\section{Letters to the editor should be sent to: notices-letters@ams.org}

Additions to the Math Calendar should be submitted at: www.ams.org/cgi-bin/mathcal/mathcal-submit.pl

To make suggestions for additions to other sections, and for full contact information, see www.ams.org/publications/ journals/notices/noticescontact

[Notices of the American Mathematical Society (ISSN 0002-9920) is published monthly except bimonthly in June/July by the American Mathematical Society at 201 Charles Street, Providence, RI 02904-2213 USA, GST No. 121892046 RT ${ }^{\star \star \star \star}$. Periodicals postage paid at Providence, RI, and additional mailing offices. POSTMASTER: Send address change notices to Notices of the American Mathematical Society, P.O. Box 6248, Providence, RI 02904-6248 USA.] Publication here of the Society's street address and the other bracketed information is a technical requirement of the US Postal Service.

(c) Copyright 2018 by the American Mathematical Society. All rights reserved.

Printed in the United States of America. The paper used in this journal is acid-free and falls within the guidelines established to ensure permanence and durability. 


\section{The On-Line Encyclopedia of Integer Sequences}

PA

Neil J. A. Sloane 


\section{Introduction}

The OEIS ${ }^{\circledast}$ (or On-Line Encyclopedia of Integer Sequences $\left.{ }^{\circledR}\right)^{1}$ is a freely accessible database of number sequences, now in its 54th year, and online since 1995. It contains over 300,000 entries, and for each one gives a definition, properties, references, computer programs, tables, etc., as appropriate. It is widely referenced: a webpage ${ }^{2}$ lists over 6, 000 works that cite it, and often say things like "this theorem would not exist without the help of the OEIS." It has been called one of the most useful mathematical sites in the Web.

The main use is to serve as a dictionary or fingerprint file for identifying number sequences (and when you find the sequence you are looking for, you will understand why the OEIS is so popular). If your sequence is not recognized, you see a message saying that if the sequence is of general interest, you should submit it for inclusion in the database. The resulting queue of new submissions is a continual source of lovely problems.

I described the OEIS in a short article in the September 2003 issue of these Notices. The most significant changes since then took place in 2009, when a nonprofit foundation $^{3}$ was set up to own and maintain the OEIS, and in 2010 when the OEIS was moved off my home page at AT\&T Labs to a commercial host. The format has also changed: since 2010 the OEIS has been a refereed "wiki." Four people played a crucial role in the transition: Harvey P. Dale and Nancy C. Eberhardt helped set up the Foundation, Russell S. Cox wrote the software, and David L. Applegate helped move the OEIS. The OEIS would probably not exist today but for their help.

All submissions, of new sequences and updates, are now refereed by volunteer editors. One of the rewards of being an editor is that you see a constant flow of new problems, often submitted by nonmathematicians, which frequently contain juicy-looking questions that are begging to be investigated.

This article will describe a selection of recent sequences, mostly connected with unsolved problems.

Sequences in the OEIS are identified by a 6-digit number prefixed by A. A000001 is the number of groups of order $n$, $\mathrm{A} 000002$ is Kolakoski's sequence, and so on. When we were approaching a quarter of a million entries, the editors voted to decide which sequence would become A250000. The winner was the Peaceable Queens sequence, described in the next section, and the runner-up was the "circles in the plane" sequence A250001 discussed after that. The $n$th term of the sequence under discussion is usually denoted by $a(n)$.

Neil J. A. Sloane is president of The OEIS Foundation, Inc. and a visiting scientist at Rutgers University. His email address is njas1oane@gmai1.com.

1 oeis.org.

2 oeis.org/wiki/Works_Citing_OEIS.

${ }^{3}$ The OEIS Foundation, Inc., oeisf.org.

For permission to reprint this article, please contact: reprint-permission@ams.org.

DOI: http://dx.doi.org/10.1090/noti1734

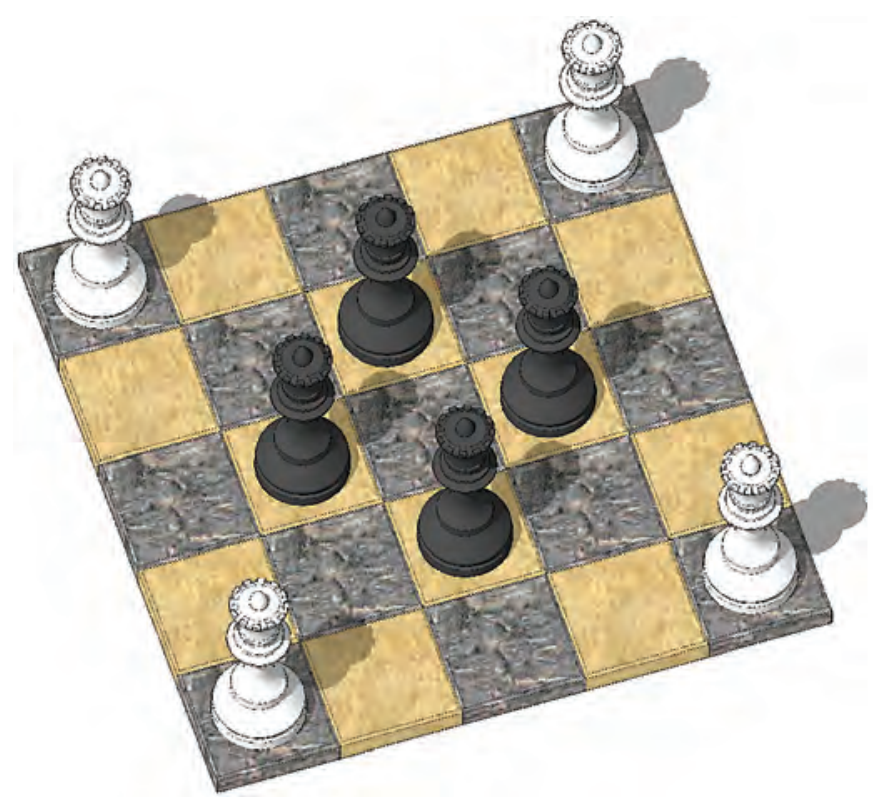

Figure 1. One of three solutions to the Peaceable Queens problem on a $5 \times 5$ board, illustrating $a(5)=4$.

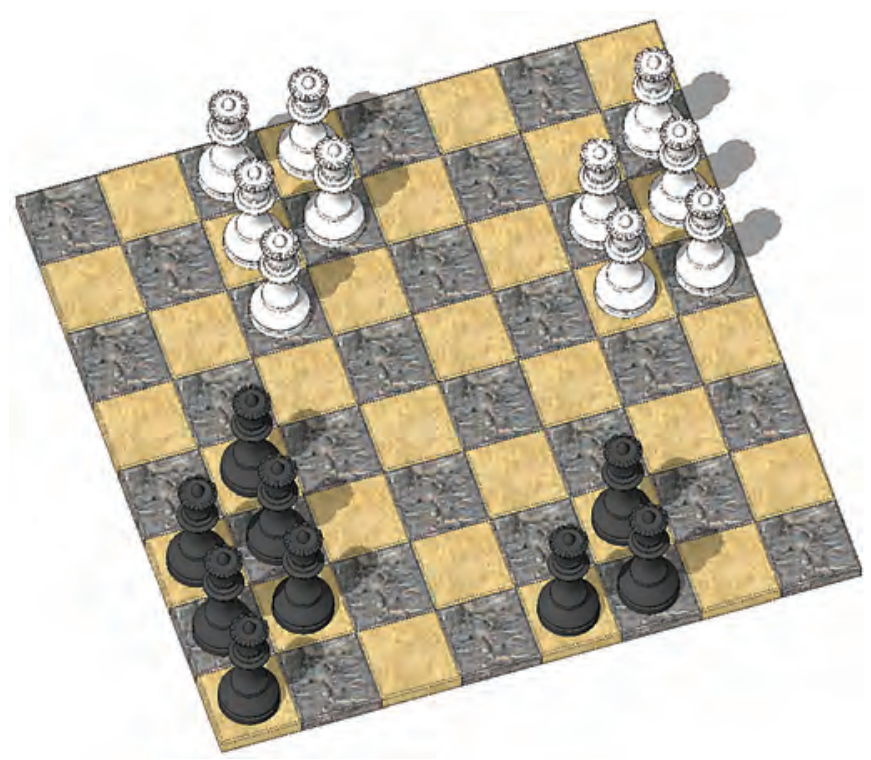

Figure 2. A solution to the Peaceable Queens problem on an $8 \times 8$ board, illustrating $a(8)=9$. (There are actually 10 white queens here but only 9 count since the numbers of white and black queens must be equal. Any one of the white queens could be omitted.)

\section{Peaceable Queens}

In $\mathrm{A} 250000, a(n)$ is the maximal number $m$ such that it is possible to place $m$ white queens and $m$ black queens on an $n \times n$ chess board so that no queen attacks a queen of the opposite color. These are peaceable queens. This is a fairly new problem with some striking pictures, 


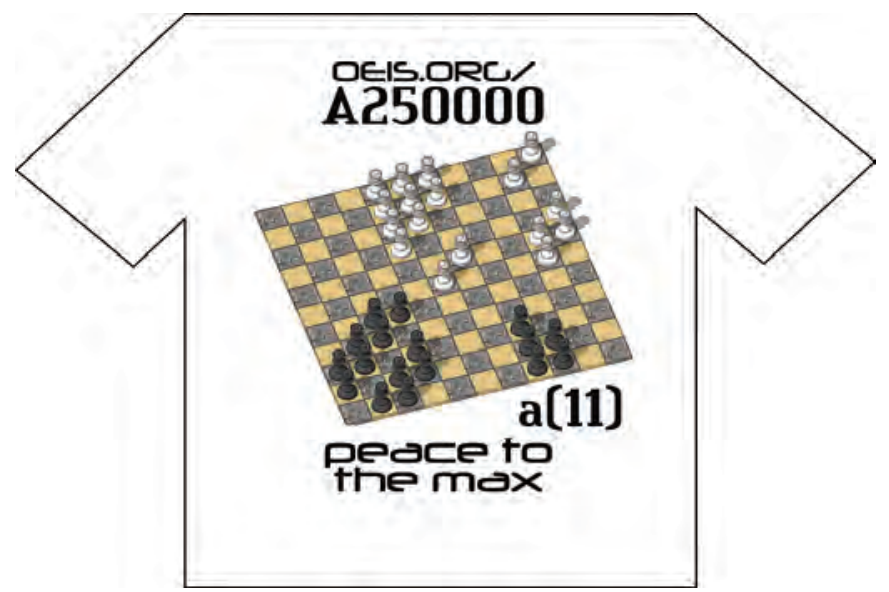

Figure 3. A solution to the Peaceable Queens problem on an $11 \times 11$ board, illustrating $a(11)=17$.

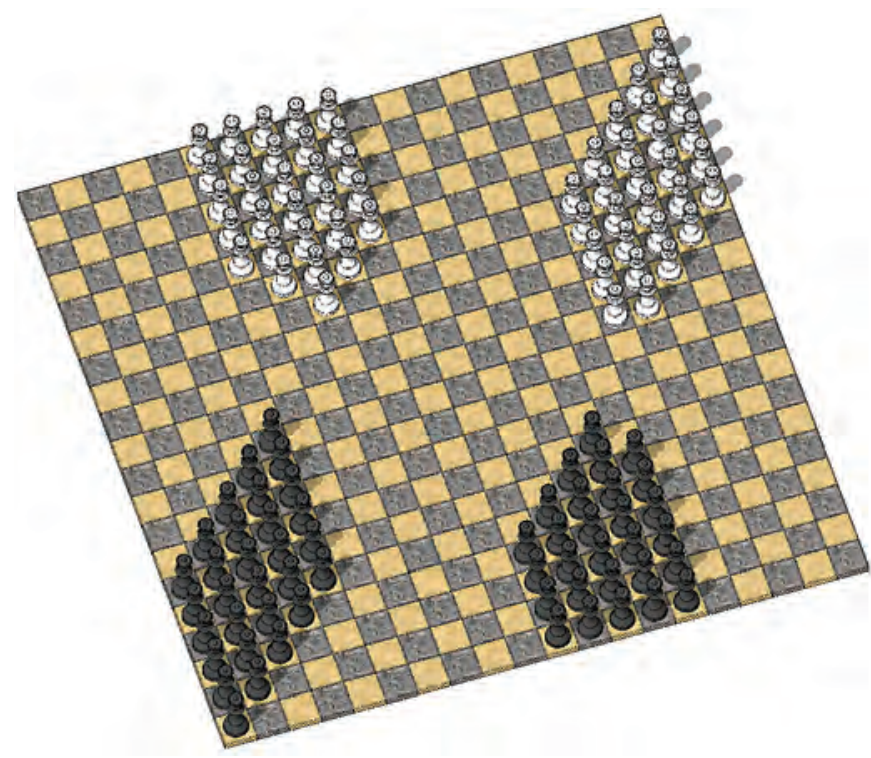

Figure 4. A conjectured solution to the Peaceable Queens problem on a $20 \times 20$ board, found by Bob Selcoe, showing that $a(20) \geq 58$.

an interesting conjecture, and a satisfactorily nonviolent theme. It was posed by Robert A. Bosch in 1999, as a variation on the classical problem of finding the number of ways to place $n$ queens on an $n \times n$ board so that they do not attack each other (A000170). It was added to the OEIS in 2014 by Donald E. Knuth, and a number of people have contributed to the entry since then. Only thirteen terms are known:

$\begin{array}{cccccccccrrrrr}n: & 1 & 2 & 3 & 4 & 5 & 6 & 7 & 8 & 9 & 10 & 11 & 12 & 13 \\ a(n): & 0 & 0 & 1 & 2 & 4 & 5 & 7 & 9 & 12 & 14 & 17 & 21 & 24\end{array}$

Figures 1-4 show examples of solutions for $n=5,8,11$ and (conjecturally) 20.

For larger values of $n$, the best solutions presently known were found by Benoit Jubin and concentrate the queens into four pentagonal regions, as shown in Figure 5 (and generalize the arrangement shown in Figure 4). This construction gives a lower bound of $\left[7 n^{2} / 48\right]$, a formula which in fact matches all the best arrangements known so far except $n=5$ and 9. It would be nice to know if this construction really does solve the problem!

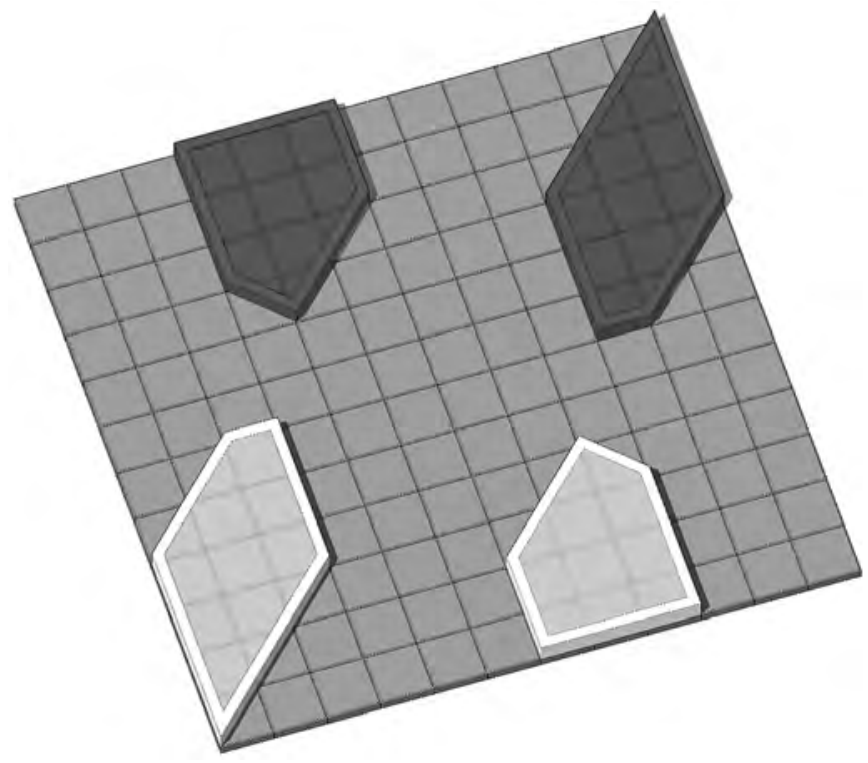

Figure 5. A general construction for the Peaceable Queens problem found by Benoît Jubin, showing that for large $n, a(n) \geq\left\lfloor 7 n^{2} / 48\right\rfloor$, a formula which might be exact for all $n>9$.

\section{Circles in the Plane}

The runner-up in the competition for $\underline{\mathrm{A} 250000}$ is now A250001: here $a(n)$ is the number of ways to draw $n$ circles in the affine plane. Two circles must be disjoint or meet in two distinct points (tangential contacts are not permitted), and three circles may not meet at a point. ${ }^{4}$ The sequence was proposed by Jonathan Wild, a professor of music at McGill University, who found the values $a(1)=1, a(2)=3, a(3)=14, a(4)=173$, and, jointly with Christopher Jones, $a(5)=16951$ (see Figures 6-8).

Wild and Jones have found that there are complications which first appear when five circles are being considered: here there are arrangements which theoretically could exist if one considered only the intersections between circles, but which cannot actually be drawn using circles. For example, start with four circles arranged in a chain, each one overlapping its two neighbors, and label the overlaps a, b, c, d (see Figure 9). Suppose we try to add a fifth circle that meets all four circles but avoids their overlaps, encloses overlaps $b$ and d, but does not enclose overlaps a or c. This can be drawn if the fifth circle

\footnotetext{
${ }^{4}$ The circles may have different radii. Two arrangements are considered the same if one can be continuously changed to the other while keeping all circles circular (although the radii may be continuously changed), without changing the multiplicity of intersection points, and without a circle passing through an intersection point. Turning the whole configuration over is allowed.
} 


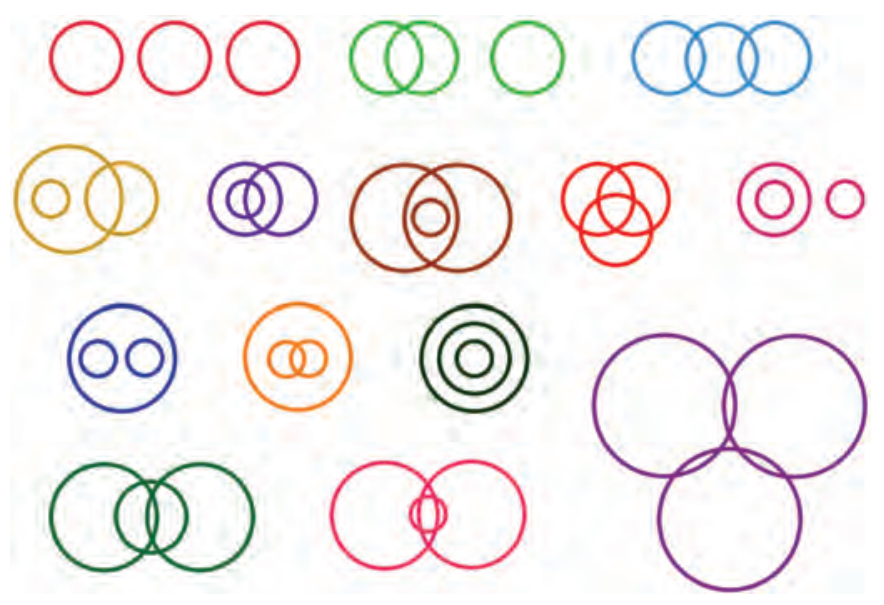

Figure 6. The fourteen ways to draw three circles in the affine plane.

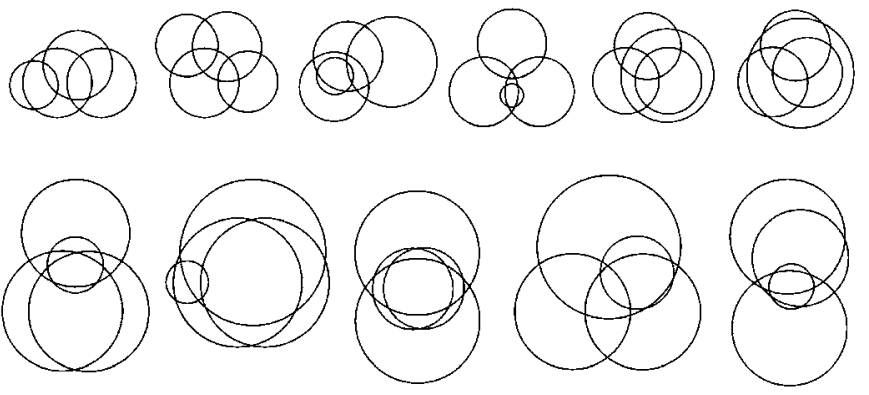

Figure 7. Eight of the 173 ways to draw four circles. For the full set of 173 drawings, see $\mathbf{A 2 5 0 0 0 1}$.

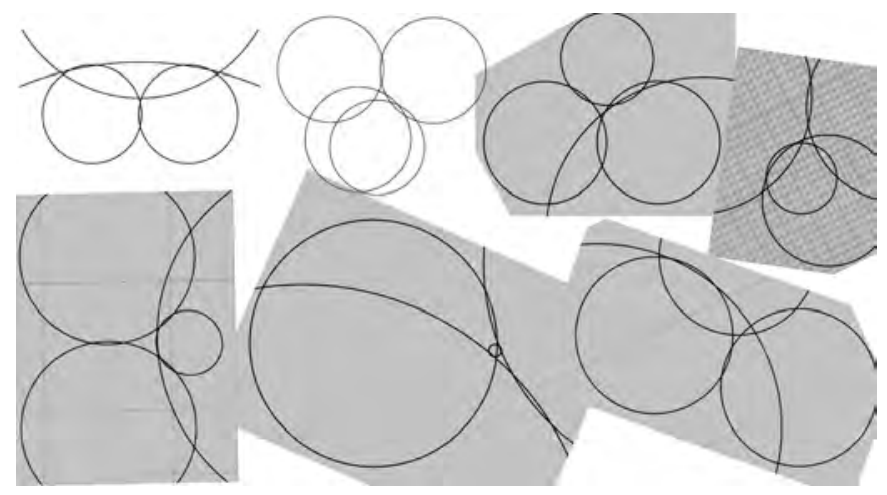

Figure 8. Seven further ways (out of 173) to draw four circles.

is flattened to an ellipse, but it can be shown that the arrangement cannot be realized with five circles. There are twenty-six such unrealizable arrangements of five circles, which can be ruled out by ad hoc arguments.

The delicate configurations like those in Figure 8 are very appealing. It would be interesting to see all 17142 arrangements of five or fewer circles displayed along the Great Wall of China.

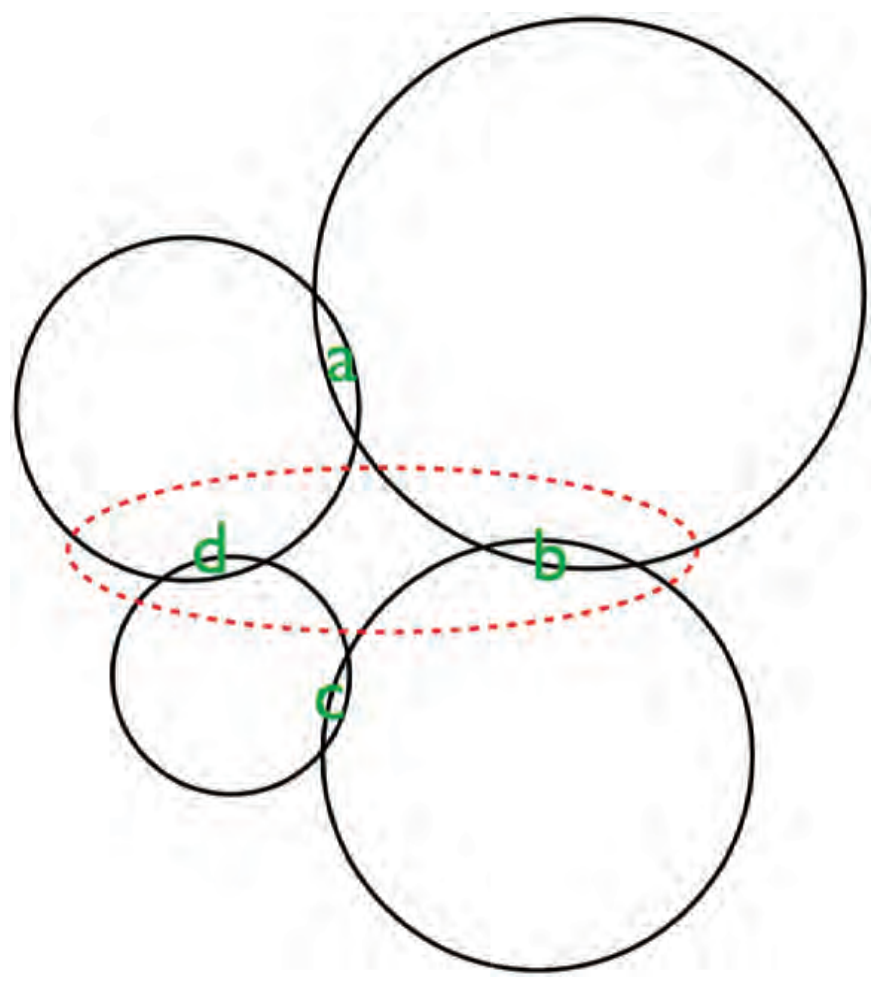

Figure 9. A hypothetical arrangement of five circles that can only be realized if one or more of the circles is distorted.

\section{Lexicographically Earliest Cube-Free Binary Sequence}

There is an obvious way to sort integer sequences $a(1), a(2), a(3), a(4), \ldots$ into lexicographic order. A number of recent entries in the OEIS are defined to be the lexicographically earliest sequence of nonnegative or positive integers satisfying certain conditions.

For example, one of the first results in the subject now called "Combinatorics on Words" was Axel Thue's 1912 theorem that the "Thue-Morse sequence"

$$
\begin{array}{r}
T=0,1,1,0,1,0,0,1,1,0,0,1, \\
0,1,1,0,1,0,0,1,0,1,1,0,0,1, \ldots
\end{array}
$$

(A010060) contains no substring of the form $X X X$, that is, $T$ is cube-free. $T$ can be defined as a fixed point of the mapping $0 \rightarrow 01,1 \rightarrow 10$; alternatively, by taking $a(n)$ to be the parity of the number of $1 \mathrm{~s}$ in the binary expansion of $n$. One hundred and five years later, David W. Wilson asked for the lexicographically earliest cube-free sequence of 0 s and 1s. Using a back-tracking algorithm, he found what appear to be the first 10000 terms, which begin

(1) $0,0,1,0,0,1,0,1,0,0,1,0,0,1$,

$$
1,0,0,1,0,0,1,0,1,0,0,1, \ldots .
$$

This is now $\mathrm{A} 282317$. 
There is no difficulty in showing that the sequence exists. $^{5}$ To see this, make the set $S$ of all infinite binary sequences $a=(a(1), a(2), \ldots)$ into a metric space by defining $d(a, b)$ to be 0 if $a=b$, or $2^{-i}$ if $a$ and $b$ first differ at position $i$. This identifies $S$ with the Cantor set in $[0,1)$. The subset $F \subset S$ of infinite cube-free sequences is nonempty and has an infimum $c$ say. It is easy to show that the complement $S \backslash F$, sequences that contain a cube, is an open set in this topology, so $F$ is closed and $c \in F$.

So far only the first 999 terms of A282317 have been verified to be correct (by showing that there is at least one infinite cube-free sequence with that beginning). The rest of the 10000 terms are only conjectural. It would be nice to know more. In particular, does this sequence have an alternative construction? There is no apparent formula or recurrence, which seems surprising.

\section{The EKG and Yellowstone Sequences}

To continue the "lexicographically earliest" theme, many recent entries in the OEIS are defined to be the lexicographically earliest sequence $a(1), a(2), \ldots$ of distinct positive integers satisfying certain divisibility conditions.

The first task here is usually to show that there are no missing numbers, i.e., that the sequence is a permutation of the positive integers. Sequences of this type were studied in a 1983 paper by Erdós, Freud, and Hegyvári, which included the examples A036552 $(a(2 n)=$ smallest missing number, $a(2 n+1)=2 a(2 n))$ and $\mathrm{A} 064736(a(2 n+2)=$ smallest missing number, $a(2 n+1)=a(2 n) \cdot a(2 n+2))$. For these two it is clear that there are no missing numbers. This is less obvious, but still true, for Jonathan Ayres's EKG sequence, A064413, defined to be the lexicographically earliest sequence of distinct positive integers such that

$$
\operatorname{gcd}(a(n-1), a(n))>1 \text { for all } n \geq 3 .
$$

This begins

$$
\begin{aligned}
1,2,4,6,3,9,12,8,10,5,15,18, \\
14,7,21,24,16,20,22,11,33,27, \ldots .
\end{aligned}
$$

The proof that it is a permutation is omitted-it is similar to the proof for the Yellowstone sequence given below.

Next, one can investigate the rate of growth. In the case of $\underline{\mathrm{A} 064413}$, the points appear to lie roughly on three curved lines (Figure 10), although the following conjecture of Lagarias, Rains, and Sloane (2002) is still open.

\section{Plot of $\mathbf{A 0 6 4 4 1 3}$}

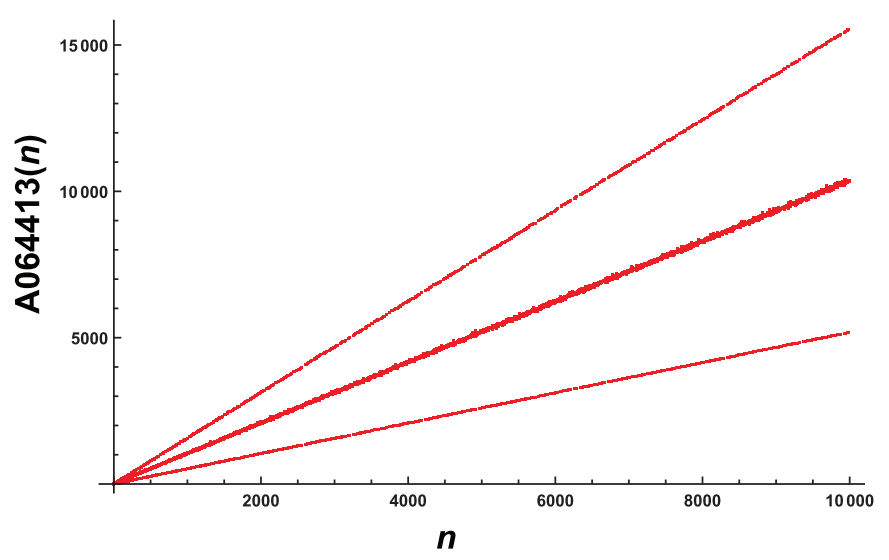

Figure 10. The first 10000 terms of the EKG sequence, so named because locally this graph resembles an EKG. Every number appears exactly once.

Conjecture 1. In the EKG sequence A064413, if $a(n)$ is neither a prime nor three times a prime then

$$
a(n) \sim n\left(1+\frac{1}{3 \log n}\right)
$$

if $a(n)$ is a prime then

$$
a(n) \sim \frac{1}{2} n\left(1+\frac{1}{3 \log n}\right) ;
$$

and if $a(n)$ is 3 times a prime then

$$
a(n) \sim \frac{3}{2} n\left(1+\frac{1}{3 \log n}\right) .
$$

Furthermore, if the sequence is a permutation, one can also try to study its cycle structure. However, this often leads to very difficult questions, similar to those encountered in studying the Collatz conjecture, and we can't do much more than collect experimental data. Typically there is a set of finite cycles, and one or more apparently infinite cycles, but we can't prove that the apparently infinite cycles really are infinite, nor that they are distinct. See the entries for $\underline{\mathrm{A} 064413}$ and $\underline{\mathrm{A} 098550}$ for examples.

The definition of the Yellowstone sequence (Reinhard Zumkeller, 2004, A098550, [1]) is similar to that of the EKG sequence, but now the requirement is that, for $n>3$, $\operatorname{gcd}(a(n-2), a(n))>1$ and $\operatorname{gcd}(a(n-1), a(n))=1$.

This begins

$$
\begin{array}{r}
1,2,3,4,9,8,15,14,5,6,25,12,35, \\
16,7,10,21,20,27,22,39,11, \ldots .
\end{array}
$$

Figure 11 shows terms $a(101)=47$ through $a(200)=$ 279 , with successive points joined by lines.

Theorem 2. The Yellowstone sequence $\underline{098550}$ is a permutation of the positive integers.

\footnotetext{
${ }^{5}$ Thanks to Jean-Paul Allouche for this argument.
} 
The proof is typical of the arguments used to prove that several similar sequences are permutations, including the EKG sequence above.

Proof. There are several steps.

(i) The sequence is infinite. (For $p a(n-2)$ is always a candidate for $a(n)$, where $p$ is a prime larger than any divisor of $a(i), i<n$.)

(ii) There are infinitely many different primes that divide the terms of the sequence. (If not, there is a prime $p$ such that all terms are products of primes less than $p$. Using (i), find a term $a(n)>p^{2}$, and let $q$ be a common prime factor of $a(n-2)$ and $a(n)$. But now $p q<p^{2}<a(n)$ is a smaller candidate for $a(n)$, a contradiction.)

(iii) For any prime $p$, some term is divisible by $p$. (For if not, no prime $q>p$ can divide any $a(n)$ : if $a(n)=k q$ is the first multiple of $q$ to appear, $k p$ would be a smaller candidate for $a(n)$. This contradicts (ii).)

(iv) For any prime $p, p$ divides infinitely many terms. (If not, let $p^{i}$ be larger than any multiple of $p$ in the sequence, and choose a prime $q>p^{i}$. Again we obtain a contradiction.)

(v) Every prime $p$ is a term in the sequence. (Suppose not, and using (i), choose $n_{0}$ such that $a(n)>p$ for all $n>n_{0}$. Using (iv), find $a(n)=k p, k>1$, for some $n>n_{0}$. But then $a(n+2)=p$, a contradiction.)

(vi) All numbers appear. For if not, let $k$ be the smallest missing number, and choose $n_{0}$ so that all of $1, \ldots, k-1$ have occurred in $a(1), \ldots, a\left(n_{0}\right)$. Let $p$ be a prime dividing $k$. Since, by (iv), $p$ divides infinitely many terms, there is a number $n_{1}>n_{0}$ such that $\operatorname{gcd}\left(a\left(n_{1}\right), k\right)>1$. This forces

$$
\operatorname{gcd}(a(n), k)>1 \text { for all } n \geq n_{1} .
$$

(If not, there would be some $j \geq n_{1}$ where $\operatorname{gcd}(a(j), k)>1$ and $\operatorname{gcd}(a(j+1), k)=1$, which would lead to $a(j+2)=$ $k$.) But (2) is impossible, because we know from (v) that infinitely many of the $a(n)$ are primes.

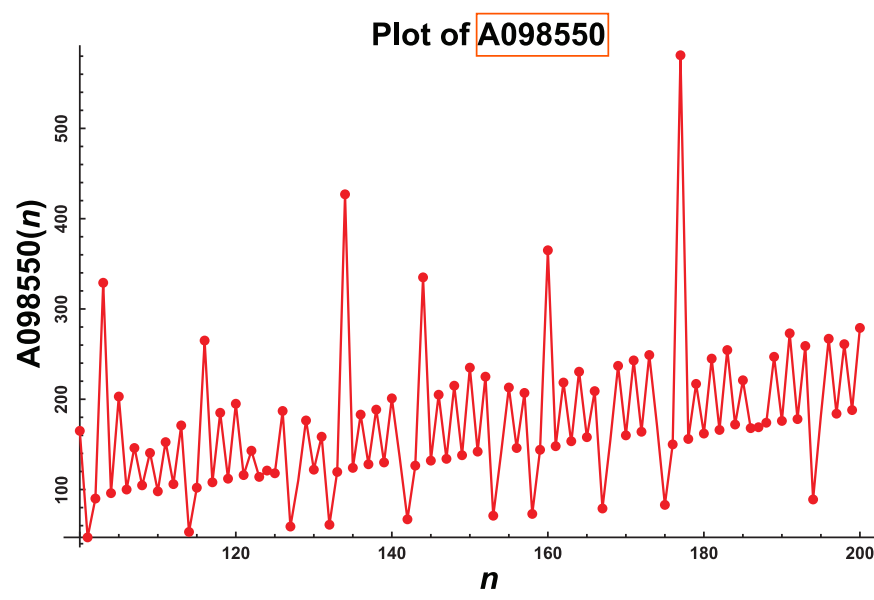

Figure 11. Plot of terms $a(101)$ through $a(200)$ of the Yellowstone sequence. The sequence has a downward spike to $a(n)$ when $a(n)$ is a prime, and larger upward spikes (the "geysers," which suggests the name for this sequence) two steps later.
The growth of this sequence is more complicated than that of the EKG sequence. Figure 12 shows the first 300,000 terms, without lines connecting the points. The points appear to fall on or close to a number of distinct curves. There is a conjecture in $[1$, p. 5] that would explain these curves.

\section{Three Further Lexicographically Earliest Sequences}

Here are three further examples of this type, all of which are surely permutations of the positive integers. For the first there is a proof, for the second there is "almost" a proof, but the third may be beyond reach.

The first (Leroy Quet, 2007, A127202) is the lexicographically earliest sequence of distinct positive integers such that

$\operatorname{gcd}(a(n-1), a(n)) \neq \operatorname{gcd}(a(n-2), a(n-1)) \quad$ for $n \geq 3$. It begins

$$
\begin{array}{r}
1,2,4,3,6,5,10,7,14,8,9,12,11,22, \\
13,26,15,18,16,17,34,19, \ldots .
\end{array}
$$

For the second (Rémy Sigrist, 2017, A280864), the definition is

\section{if a prime $p$ divides $a(n)$, then it divides}

$$
\text { exactly one of } a(n-1) \text { and } a(n+1) \text {, for } n \geq 2 \text {, }
$$

and the initial terms are

$$
1,2,4,3,6,8,5,10,12,9,7,14,16,11 \text {, }
$$

$$
22,18,15,20,24,21,28,26, \ldots \text {. }
$$

The proof that the first is a permutation is similar to that for the Yellowstone sequence, although a bit more involved (see A127202). The second struck me as one of

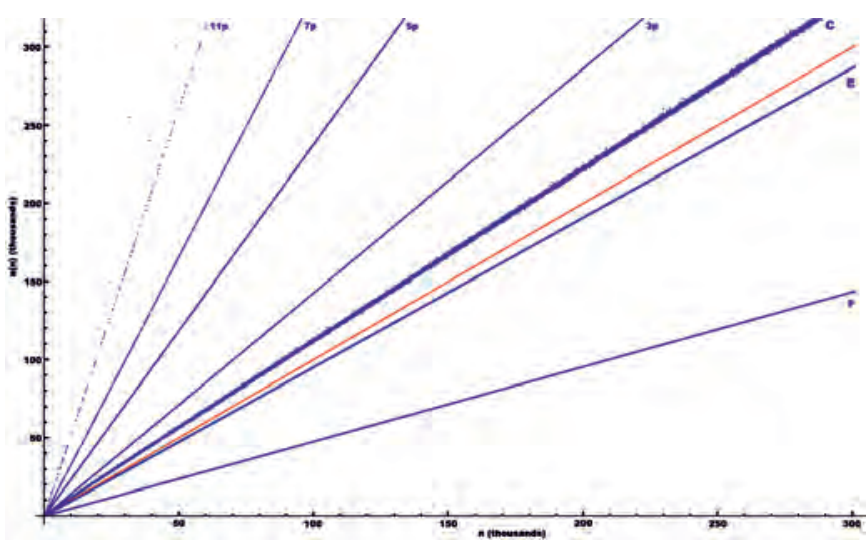

Figure 12. Scatterplot of the first 300,000 terms of the Yellowstone sequence. The primes lie on the lowest line (labeled "p"), the even numbers on the second line ("E"), the majority of the odd composite numbers on the third line ("C"), and the $3 p, 5 p, 7 p$, $11 p, \ldots$ points on the higher lines. The lines are not actually straight, except for the red line $f(x)=x$, which is included for reference. 
those "drop everything and work on this" problems that are common hazards when editing new submissions to the OEIS. However, after several months, I could prove that every prime and every even number appears, and that if $p$ is an odd prime then there are infinitely many odd multiples of $p$ (see A280864 for details), but I could not prove that every odd number appears. The missing step feels like it is only a couple of cups of coffee away, and I'm hoping that some reader of this article will complete the proof.

The third example (Henry Bottomley, 2000, A055265) is the lexicographically earliest sequence of distinct positive integers such that $a(n-1)+a(n)$ is a prime for $n \geq 2$ :

$$
\begin{aligned}
& 1,2,3,4,7,6,5,8,9,10,13,16,15 \\
& 14,17,12,11,18,19,22,21,20, \ldots .
\end{aligned}
$$

The terms appear to lie on or near the line $a(n)=n$, but the proof that every number appears may be difficult because it involves the gaps between the primes.

\section{Two-Dimensional Lexicographically Earliest Arrays}

The OEIS is primarily a database of sequences $\left(a_{n}, n \geq n_{0}\right)$. However, triangles of numbers are included by reading them by rows. Pascal's triangle becomes $1,1,1,1,2,1,1,3,3,1,1,4,6,4,1, \ldots$, which (without the extra spaces) is A007318. Doublyindexed arrays $\left(T_{m, n}, m \geq m_{0}, n \geq n_{0}\right)$ are converted to sequences by reading them by antidiagonals (in either the upwards or downwards directions, or both). So an array $\left(T_{m, n}, m \geq 0, n \geq 0\right)$ might become $T_{0,0}, T_{1,0}, T_{0,1}, T_{2,0}, T_{1.1}, T_{0,2}, \ldots$. For example, the table of Nim-sums $m \oplus n$ :

$\begin{array}{lllllllll}0 & 1 & 2 & 3 & 4 & 5 & 6 & 7 & \ldots \\ 1 & 0 & 3 & 2 & 5 & 4 & 7 & 6 & \ldots \\ 2 & 3 & 0 & 1 & 6 & 7 & 4 & 5 & \ldots \\ 3 & 2 & 1 & 0 & 7 & 6 & 5 & 4 & \ldots \\ 4 & 5 & 6 & 7 & 0 & 1 & 2 & 3 & \cdots \\ 5 & 4 & 7 & 6 & 1 & 0 & 3 & 2 & \ldots \\ 6 & 7 & 4 & 5 & 2 & 3 & 0 & 1 & \cdots \\ 7 & 6 & 5 & 4 & 3 & 2 & 1 & 0 & \ldots \\ . & . & . & . & . & . & . & . & \ldots\end{array}$

produces the sequence $\underline{\underline{A 003987}}$ :

$$
\begin{aligned}
0,1,1,2,0,2,3,3,3,3,4,2,0,2,4, \\
5,5,1,1,5,5,6,4,6,0,6,4,6, \ldots .
\end{aligned}
$$

Doubly-indexed doubly-infinite arrays $\left(T_{m, n}, m \in \mathbb{Z}\right.$, $n \in \mathbb{Z}$ ) can become sequences by reading them in a spiral around the origin, in say a counter-clockwise direction: $T_{0,0}, T_{1,0}, T_{1,1}, T_{0,1}, T_{-1,1}, T_{-1,0}, T_{-1,-1}, T_{0,-1}, \ldots$ (cf. Figure 13).

There are many "lexicographically earliest" versions of these arrays. For example, the Nim-sum array (3) has an equivalent definition: scan along upwards antidiagonals, filling in each cell with the smallest nonnegative number that is neither in the row to the left of that cell nor in the column above it.
A variation on the Nim-sum array was proposed by Alec Jones in 2016, as a kind of "infinite Sudoku array." This array $\left(T_{m, n}, m \geq 0, n \geq 0\right)$ is to be filled in by upwards antidiagonals, always choosing the smallest positive integer such that no row, column, diagonal, or antidiagonal contains a repeated term. The top left corner of the array is:

$\begin{array}{ccccccccc}1 & 3 & 2 & 6 & 4 & 5 & 10 & 11 & \ldots \\ 2 & 4 & 5 & 1 & 8 & 3 & 6 & 12 & \ldots \\ 3 & 1 & 6 & 2 & 9 & 7 & 5 & 4 & \ldots \\ 4 & 2 & 3 & 5 & 1 & 8 & 9 & 7 & \ldots \\ 5 & 7 & 1 & 4 & 2 & 6 & 3 & 15 & \cdots \\ 6 & 8 & 9 & 7 & 5 & 10 & 4 & 16 & \cdots \\ 7 & 5 & 4 & 3 & 6 & 14 & 8 & 9 & \ldots \\ 8 & 6 & 7 & 9 & 11 & 4 & 13 & 3 & \cdots \\ . & . & . & . & . & . & . & . & \cdots\end{array}$

The resulting sequence (A269526 is

$$
\begin{aligned}
1,2,3,3,4,2,4,1,5,6,5,2,6,1,4 \\
6,7,3,2,8,5,7,8,1,5,9,3,10, \ldots .
\end{aligned}
$$

This array has many interesting properties. If we subtract 1 from each entry, the entries are the Nim-values for a game played with two piles of counters, of sizes $m$ and $n$, and reminiscent of Wythoff's game (see A004481, $\underline{\mathrm{A} 274528}$.

But the main question about the array (4) is, are the individual rows, columns, and diagonals of this array permutations of $\mathbb{N}$ ? (The antidiagonals are obviously not,

$$
\begin{aligned}
& 9 \leftarrow 16 \leftarrow 2 \leftarrow 4 \leftarrow 7 \leftarrow 14 \leftarrow 11 \leftarrow 12 \leftarrow 1 \leftarrow 5 \leftarrow 8 \\
& \downarrow \\
& 178 \leftarrow 15 \leftarrow 14 \leftarrow 13 \leftarrow 12 \leftarrow 9 \leftarrow 10 \leftarrow 6 \leftarrow 7 \quad 3 \\
& \downarrow \downarrow \downarrow \downarrow 17 \% \\
& 124 \leftarrow 11 \leftarrow 10 \leftarrow 3 \leftarrow 8 \leftarrow 7 \leftarrow 9 \quad 13 \quad 15 \\
& \downarrow \begin{array}{llllllll}
1 & \downarrow & \downarrow & \uparrow & \uparrow & \uparrow
\end{array} \\
& 897 \quad 3 \leftarrow 5 \leftarrow 6 \leftarrow 1 \leftarrow 2 \quad 4 \quad 12 \quad 11
\end{aligned}
$$

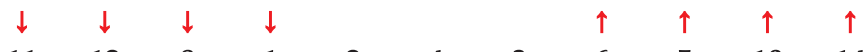

$$
\begin{aligned}
& \begin{array}{llllllllll}
11 & 12 & 8 & 1 & 2 \leftarrow 4 & \leftarrow 3 & 6 & 5 & 10 & 14
\end{array}
\end{aligned}
$$

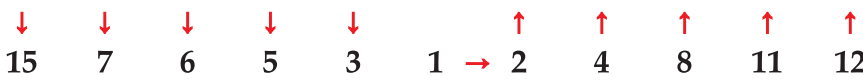

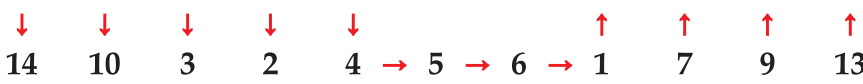

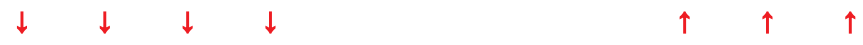

$$
\begin{aligned}
& \begin{array}{lllllllllllll}
7 & 11 & 9 & 6 & \rightarrow 1 \rightarrow 2 & \rightarrow 4 & \rightarrow 5 & \rightarrow 3 & 8 & 10
\end{array}
\end{aligned}
$$

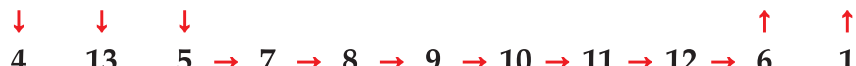

$$
\begin{aligned}
& \begin{array}{ccccccc}
4 & 13 & 5 \rightarrow 7 \rightarrow 8 \rightarrow 9 \rightarrow 10 \rightarrow 11 \rightarrow 12 \rightarrow 6 & 1 \\
\downarrow & \downarrow & & &
\end{array} \\
& 1214 \rightarrow 10 \rightarrow 9 \rightarrow 6 \rightarrow 13 \rightarrow 5 \rightarrow 3 \rightarrow 15 \rightarrow 16 \rightarrow 7 \\
& \downarrow \\
& 10 \rightarrow 15 \rightarrow 1 \rightarrow 12 \rightarrow 16 \rightarrow 8 \rightarrow 14 \rightarrow 13 \rightarrow 11 \rightarrow 18 \rightarrow 17
\end{aligned}
$$

Figure 13. A274640: choose the smallest positive number so that no row, column, or diagonal contains a repeat. Are the rows, columns, diagonals permutations of $\mathbb{N}$ ? 
since they are finite sequences.) It is easy to see that each column is a permutation. In column $c \geq 0$, a number $k$ will eventually be the smallest missing number and will appear in some cell in that column, unless there is a copy of $k$ to the North-West, West, or South-West of that cell. But there are at most $c$ copies of $k$ in all the earlier columns, so eventually $k$ will appear.

The rows are also permutations, although the proof is less obvious. Consider row $r \geq 0$, and suppose $k$ never appears. There are at most $r$ copies of $k$ in the earlier rows, and these can affect only a bounded portion of row $r$. Consider a cell $(r, n), n \geq 0$ large. If $k$ is not to appear in that cell, there must be a copy of $k$ in the antidiagonal to the South-West. So in the triangle bounded by row $r$, column 0 , and the antidiagonal through $(r, n)$, there must be at least $n+1-r$ copies of $k$. Imagine these $k s$ replaced by chess queens. By construction they are mutually nonattacking. But it is known ([6, Problem 252], or A274616) that on a triangular half-chessboard of side $n$, there can be at most $2 n / 3+1$ mutually nonattacking queens, which for large $n$ leads to a contradiction.

As to the diagonals, although they appear to be permutations, this is an open question. The argument using nonattacking queens breaks down because the diagonal of the half-chessboard contains only half as many squares as the sides. Even the main diagonal, A274318,

$$
\begin{aligned}
& 1,4,6,5,2,10,8,3,7,9,16,26,29, \\
& 22,20,23,28,38,12,32,46,13,14,11,15, \ldots,
\end{aligned}
$$

is not presently known to be a permutation of $\mathbb{N}$.

The spiral version of this array is even more frustrating. This array $((T(m, n), m \in \mathbb{Z}, n \in \mathbb{Z})$, A274640, proposed by Zak Seidov and Kerry Mitchell in June 2016), is constructed in a counterclockwise spiral, filling in each cell with the smallest positive number such that no row, column, or diagonal contains a repeated term (Figures 13, 14). ("Diagonal" now means any line of cells of slope \pm 1 .)

Although it seems very plausible that every row, column, and diagonal is a permutation of $\mathbb{N}$, now there are no proofs at all. The eight spokes through the center are sequences A274924 A274931. For example, the row through the central cell is

$$
\begin{aligned}
\ldots, 14,25,13,17,10,15,7,6,5,3, \\
1,2,4,8,11,12,16,9,19,24,22, \ldots,
\end{aligned}
$$

which is $\mathrm{A} 274928$ reversed followed by $\mathrm{A} 274924$. Is it a permutation of $\mathbb{N}$ ? We do not know.

\section{Fun With Digits}

Functions of the digits of numbers have always fascinated people, ${ }^{6}$ and one such function was in the news in 2017. The idea underlying this story and several related sequences is to start with some simple function $f(n)$ of

\footnotetext{
${ }^{6}$ Although in A Mathematician's Apology, G. H. Hardy, referring to the fact that 1089 and 2178 are the smallest numbers which when written backwards are nontrivial multiples of themselves ( $c f . \underline{\text { A008919) }}$, remarked that this fact was "likely to amuse amateurs," but was not of interest to mathematicians.
}

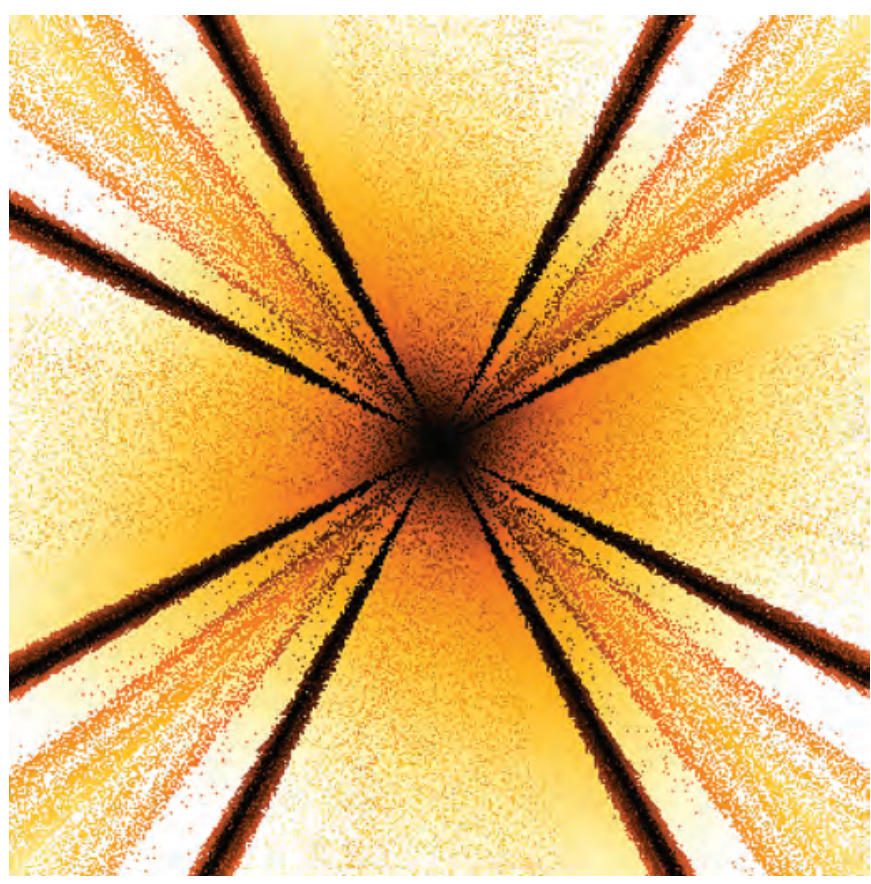

Figure 14. Colored representation of central $200 \times 200$ portion of the spiral in Figure 13: the colors represent the values, ranging from black (smallest) to white (largest).

the digits of $n$ in some base, iterate it, and watch what happens.

For the first example we write $n$ as a product of prime powers, $n=p_{1}^{e_{1}} p_{2}^{e_{2}} \cdots$ with the $p_{i}$ in increasing order, and define $f(n)$ to be the decimal concatenation $p_{1} e_{1} p_{2} e_{2} \ldots$, where we omit any exponents $e_{i}$ that are equal to 1 . So $f(7)=f\left(7^{1}\right)=7, f(8)=f\left(2^{3}\right)=23$.

The initial values of $f(n)(\underline{\mathrm{A080670}})$ are

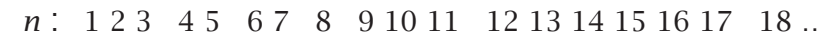

$f(n): 123225237233225112231327352417232 \ldots$

If we start with a positive number $n$ and repeatedly apply $f$, in many small cases we rapidly reach a prime (or 1). ${ }^{7}$ For example, $9=3^{2} \rightarrow 32=2^{5} \rightarrow 25=5^{2} \rightarrow$ $52=2^{2} 13 \rightarrow 2213$, a prime. Define $F(n)$ to be the prime that is eventually reached, or -1 if the iteration never reaches a prime. The value -1 will occur if the iterates are unbounded or if they enter a cycle of composite numbers. The initial values of $F(n)$ A195264) are

$$
\begin{array}{r}
1,2,3,211,5,23,7,23,2213,2213,11, \\
223,13,311,1129,233,17,17137,19, \ldots .
\end{array}
$$

$F(20)$ is currently unknown (after 110 steps the trajectory of twenty has stalled at a 192-digit number which has not yet been factored). At a DIMACS conference in October 2014 to celebrate the fiftieth anniversary of the start of what is now the OEIS, John H. Conway offered \$US1000

${ }^{7}$ In what follows we will tacitly assume $n \geq 2$, to avoid having to repeatedly say "(or 1$)$." 
for a proof or disproof of his conjecture that the iteration of $f$ will always reach a prime.

However, in June 2017 James Davis found a number $D_{0}=13532385396179$ whose prime factorization is $13 \cdot 53^{2} \cdot 3853 \cdot 96179$, and so clearly $f\left(D_{0}\right)=D_{0}$ and $F\left(D_{0}\right)=-1$.

The method used by James Davis to find $D_{0}$ is quite simple. Suppose $n=m \cdot p$ is fixed by $f$, where $p$ is a prime greater than all the prime factors of $m$. Then $f(n)=f(m) 10^{y}+p$, where $y$ is the number of digits in $p$. From $f(n)=n$ we have $p=\frac{f(m) 10^{y}}{m-1}$. Assuming $p \neq 2,5$, this implies that $p$ divides $f(m)$, and setting $x=f(m) / p$, we find that $m=x 10^{y}+1$ with $p=\frac{f(m)}{x}$ prime. A computer easily finds the solution $x=1407, y=5, m=140700001$, $p=96179$, and so $n=D_{0}$.

No other composite fixed points are known, and David J. Seal has recently shown that there is no composite fixed point less than $D_{0}$. It is easy, however, to find numbers whose trajectory under $f$ ends at $D_{0}$, by repeatedly finding a prime prefix of the previous number, as shown by the example ${ }^{8} D_{1}=13^{532385396179}$ with $f\left(D_{1}\right)=D_{0}$. So presumably there are infinitely many $n$ with $F(n)=-1$.

Consideration of the analogous questions in other bases might have suggested that counterexamples to Conway's question could exist. We will use subscripts to indicate the base (so $4_{10}=100_{2}$ ). The base- 2 analog of $f, f_{2}$ (say), is defined by taking $f_{2}\left(p_{1}^{e_{1}} p_{2}^{e_{2}} \cdots\right)$ to be the concatenation $p_{1} e_{1} p_{2} e_{2} \ldots$, as before (again omitting any $e_{i}$ that are 1), except that now we write the $p_{i}$ and $e_{i}$ in base 2 and interpret the concatenation as a base-2 number. For example, $f_{2}(8)=f_{2}\left(2^{3}\right)=1011_{2}=11_{10}$.

The initial values of $f_{2}(n)(\underline{\underline{A} 230625})$ are

$n: \quad 123 \quad 45 \quad 67 \quad 8 \quad 9101112131415161718 \ldots$

$f_{2}(n): 1231051171114211143132329201746 \ldots$

and the base-2 analog of $F, F_{2}$ A230627) is the prime (or 1 ) that is reached when $f_{2}$ is repeatedly applied to $n$, or -1 if no prime (or 1 ) is reached:

$$
1,2,3,31,5,11,7,11,23,31,11,43,13,23,29,251,17,23, \ldots \text {. }
$$

Now there is a fairly small composite fixed point, namely 255987, found by David J. Seal. Sean A. Irvine and Chai Wah Wu have also studied this sequence, and the present status is that $F_{2}(n)$ is known for all $n$ less than 12388 . All numbers in this range reach 1 , a prime, the composite number 255987 , or one of the two cycles $1007 \longleftrightarrow 1269$ or $1503 \longleftrightarrow 3751$. The numbers for which $F_{2}(n)=-1$ are $217,255,446,558, \ldots$ (A288847). Initially it appeared that 234 might be on this list, but Irvine found that after 104 steps the trajectory reaches the 51-digit prime

350743229748317519260857777660944018966290406786641.

\section{Home Primes}

A rather older problem arises if we change the definition of $f(n)$ slightly, making $f(8)=222$ rather than 23 . So if $n=p_{1} \cdot p_{2} \cdot p_{3} \cdot \ldots$, where $p_{1} \leq p_{2} \leq p_{3} \leq \ldots$, then $f(n)$ is the decimal concatenation $p_{1} p_{2} p_{3} \ldots($ A037276). In 1990, Jeffrey Heleen studied the analog of $F(n)$ for this

\footnotetext{
${ }^{8}$ Found by Hans Havermann.
}

function: that is, $F(n)$ is the prime reached if we start with $n$ and repeatedly apply $f$, or -1 if no prime is ever reached (A037274).

The trajectory of 8 now takes 14 steps to reach a prime (the individual prime factors here have been separated by spaces):

$$
\begin{aligned}
8 & \rightarrow 222 \rightarrow 2337 \rightarrow 31941 \rightarrow 33371313 \rightarrow 311123771 \\
& \rightarrow 7149317941 \rightarrow \rightarrow 22931219729 \rightarrow 112084656339 \\
& \rightarrow 3347911118189 \rightarrow 11613496501723 \rightarrow \\
& \rightarrow 97130517917327 \rightarrow 531832651281459 \\
& \rightarrow 3331113965338635107 \\
& \rightarrow 3331113965338635107,
\end{aligned}
$$

the last number being a prime.

Since $f(n)>n$ if $n$ is composite, now there cannot be any composite fixed points nor any cycles of length greater than 1 . The only way for $F(n)$ to be -1 is for the trajectory of $n$ to be unbounded. This appears to be a harder problem than the one in the previous section, since so far no trajectory has been proved to be unbounded. The first open case is $n=49$, which after 119 iterations has reached a 251-digit composite number (see A056938). The completion of the factorization for step $11 \overline{\text { took } 765}$ days by the general number field sieve, and at the time (December 2014) was one of the hardest factorizations ever completed.

\section{Power Trains}

A third choice for $f(n)$ was proposed by John H. Conway in 2007: he called it the power train map. If the decimal expansion of $n$ is $d_{1} d_{2} d_{3} \ldots d_{k}$ (with $0 \leq d_{i} \leq 9,0<d_{1}$ ), then $f(n)=d_{1}^{d_{2}} \cdot d_{3}^{d_{4}} \cdots$, ending with $\ldots \cdot d_{k}$ if $k$ is odd, or with $\ldots \cdot d_{k-1}^{d_{k}}$ if $k$ is even (A133500). We take $0^{0}$ to be 1. For example, $f(39)=3^{9}=19683, f(623)=$ $6^{2} \cdot 3=108$. Conway observed that $2592=2^{5} 9^{2}$ is a nontrivial fixed point, and asked me if there were any others. I found one more: $n=2^{46} \cdot 3^{6} \cdot 5^{10} \cdot 7^{2}=$ 24547284284866560000000000 , for which $f(n)=2^{4}$. $5^{4} \cdot 7^{2} \cdot 8^{4} \cdot 2^{8} \cdot 4^{8} \cdot 6^{6} \cdot 5^{6} \cdot 0^{0} \cdot 0^{0} \cdot 0^{0} \cdot 0^{0} \cdot 0^{0}=n$. The eleven known fixed points (including the trivial values $1, \ldots, 9)$ form A135385, and it is known that there are no further terms below $10^{100}$. Maybe this is a hint that for all of the functions $f(n)$ that have just been mentioned, there may be only a handful of genuinely exceptional values?

\section{A Memorable Prime}

If you happen to need an explicit 20-digit prime in a hurry, it is useful to remember that although $1,121=11^{2}, 12321=111^{2}, 1234321=1111^{2}, \ldots$, and $12345678987654321=111111111^{2}$ are not primes, the next term in A173426 is a prime,

\section{1.}

As David Broadhurst remarked on the Number Theory Mailing List in August 2015, this is a memorable prime! He also pointed out that on probabilistic grounds, there should be infinitely many values of $n$ such that the decimal 
concatenation of the numbers 1 up through $n$ followed by $n-1$ down through 1 is a prime. Shortly afterwards, Shyam Sunder Gupta found what is presumably the next prime in the sequence, corresponding to $n=2446$, the 17350-digit probable prime 1234567..244524462445..7654321. Serge Batalov has shown that there are no further terms with $n<60000$. What is the next term? The values 10,2446 are not enough to create an OEIS entry.

\section{A Missing Prime}

The previous question naturally led me to wonder what the first prime is in the simpler sequence (A007908):

$$
1,12,123,1234, \ldots, 12345678910,1234567891011, \ldots,
$$

formed by the decimal concatenation of the numbers 1 through $n$. In Unsolved Problems in Number Theory, Richard K. Guy reports that this question was already asked by Charles Nicol and John Selfridge. However, although the same probabilistic argument suggests that there should be an infinite number of primes of this type, not a single one is known. I asked several friends to help with the search, and as a result this task was taken up by the folks who run the GIMP (or Great Internet Mersenne Prime) search, and there is now a webpage ${ }^{9}$ that shows the current status of the search for the first prime. As of August 2017 the search seems to have stalled, the present status being that all the potential values of $n$ through 344869 failed (obviously many values of $n$ can be ruled out by congruence conditions). In this range the candidates have about two million digits. One estimate suggests that there is a probability of about 0.5 that a prime will be found with $n<10^{6}$, so it would be good to resume this search.

\section{Post's Tag System}

In his recent book Elements of Mathematics: From Euclid to Gödel ${ }^{10}$ John Stillwell mentions that Emil L. Post's tag system from the 1930s is still not understood. Post asked the following question. Take a finite string, or word, $S$ of $0 \mathrm{~s}$ and $1 \mathrm{~s}$, and if it begins with 0 , append 00 to the end of $S$ and delete the first three symbols, or if it begins with 1 , append 1101 to the end of $S$ and delete the first three symbols. When this process is iterated, eventually one of three things will happen: either $S$ will reach the empty word ( $S$ dies), $S$ will enter a loop (S cycles), or $S$ will keep growing for ever ( $S$ blows up). For example, $S=1000$ reaches the empty word $\epsilon$ at the 7th step:

$$
1000 \rightarrow 01101 \rightarrow 0100 \rightarrow 000 \rightarrow 00 \rightarrow 0 \rightarrow \epsilon,
$$

whereas 100100 enters a cycle of length six (indicated by parentheses) after 15 steps:

\footnotetext{
9 mersenneforum.org/showthread.php?t=20527.

${ }^{10}$ A superb successor to Felix Klein's 1908, Elementary Mathematics from an Advanced Standpoint.
}

(5)

$$
\begin{aligned}
100100 & \rightarrow 1001101 \rightarrow 11011101 \rightarrow 111011101 \\
& \rightarrow 0111011101 \rightarrow 101110100 \rightarrow 1101001101 \\
& \rightarrow 10011011101 \rightarrow 110111011101 \\
& \rightarrow 1110111011101 \rightarrow 01110111011101 \\
& \rightarrow 1011101110100 \rightarrow 11011101001101 \\
& \rightarrow 111010011011101 \rightarrow 0100110111011101 \\
& \rightarrow(011011101110100 \rightarrow 01110111010000 \\
& \rightarrow 1011101000000 \rightarrow 11010000001101 \\
& \rightarrow 100000011011101 \rightarrow 0000110111011101) .
\end{aligned}
$$

Post was hoping to find an algorithm which, given $S$, would determine which of these outcomes would occur. He did not succeed.

Post called this process a 'tag system.' It can be generalized by considering initial words over an alphabet of size $M$ (rather than 2), allowing any fixed set $\mathcal{A}$ of $M$ tag words to be appended (rather than 00 and 1101), and deleting some fixed number $P$ of initial symbols at each step (not necessarily 3). In 1961, Marvin Minsky showed that such a generalized tag system could simulate a Turing machine. By choosing an appropriate alphabet, an appropriate set $\mathcal{A}$ of tag words to be appended, and an appropriate value of $P$ (in fact $P=2$ will do), any computable function can be simulated. So, because of the undecidability of the Halting Problem, for general tag systems it is impossible to predict which initial words will blow up.

But what about Post's original tag system? Could this simulate a Turing machine (by encoding the problem in the initial word $S$ )? At first this seems very unlikely, but the Cook-Wolfram theorem that the one-dimensional cellular automaton defined by Rule 110 can simulate a Turing machine (by encoding the problem in the starting state) suggests that it might be possible. If it is possible, there must be some initial words that blow up (again because of the Halting Problem).

In early 2017, when I read Stillwell's book, the OEIS contained three sequences related to the original tag system, based on the work of Peter Asveld and submitted by Jeffrey Shallit: A284116, giving the maximal number of words in the 'trajectory' of any initial word $S$ of length $n$ (18 terms were known), and two sequences connected with the especially interesting starting word $\sigma_{n}$ of length $3 n$ consisting of $n$ copies of 100. A284119 $(n)$ is defined to be the number of words in the trajectory of $\sigma_{n}$ before it enters a cycle or dies, or -1 if the trajectory blows up, and A284121 ( $n$ ) is the length of the cycle, or 1 if the trajectory dies, or -1 if the trajectory blows up. For example, from (5) we see that $\mathrm{A} 284119(2)=15$ and $\mathrm{A} 284121(2)=6$. Shallit had extended Asveld's work and had found 43 terms of the two last-mentioned sequences.

I then added many further sequences based on tag systems discussed by Asveld, Liesbeth De Mol, Shigeru Watanabe, and others, and appealed to contributors to the OEIS to extend them.

The most interesting response came from Lars Blomberg, who investigated the trajectory of $\sigma_{n}$ for 
$n \leq 110$. On September 9, 2017, he reported that every $\sigma_{n}$ for $n \leq 110$ had either died or cycled after at most 13 million terms, except for $\sigma_{110}$, which after $38.10^{11}$ steps had reached a word of length $10^{7}$ and was still growing. This was exciting news! Could $\sigma_{110}$ be the first word to be discovered that blew up ${ }^{11}$ Sadly, on October 4, 2017, Blomberg reported that after 43913328040672 steps $\sigma_{110}$ had terminated in the empty word.

Figure 15 displays the remarkable graph (technically, a pin plot) of the number of steps for $\sigma_{n}$ to either die or cycle for $n \leq 200$. Figure 16 shows the lengths of the successive words in the trajectory of $\sigma_{110}$.

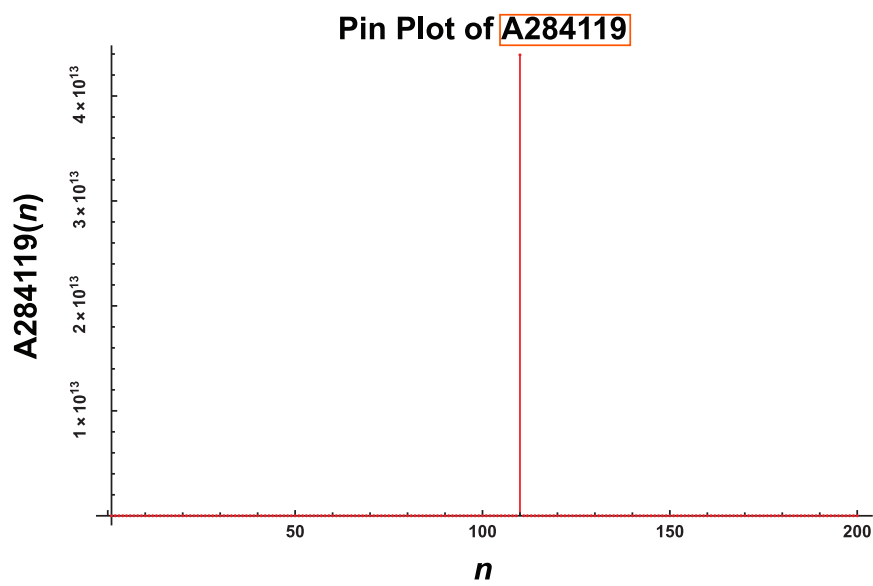

Figure 15. Pin plot illustrating Lars Blomberg's remarkable discovery that the Post tag system started at the word $(100)^{110}$ takes an exceptionally long time (43913328040672 steps) to converge.

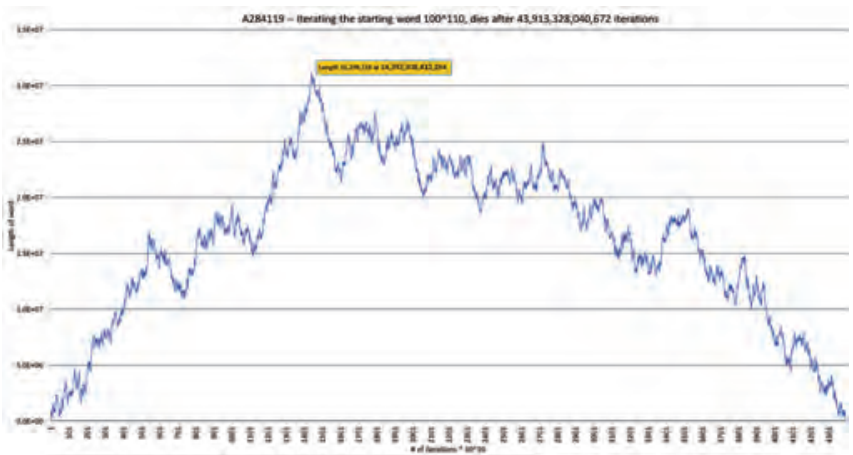

Figure 16. Lengths of successive words in trajectory of $(100)^{110}$ under the Post tag system. The numbers on the horizontal axis are spaced at multiples of $10^{12}$.

In the past six months Blomberg has continued this investigation and has determined the fate of $\sigma_{n}$ for all $n \leq 6075$. The new record-holder for the number of steps before the trajectory dies is now held by $\sigma_{4974}$, which takes 57042251906801 steps, while $\sigma_{110}$ is in second place.

\footnotetext{
${ }^{11}$ Of course the fact that the same number 110 was involved could not possibly be anything more than a coincidence.
}

Of course it is still possible that some initial word $S$, not necessarily of the form $\sigma_{n}$, will blow up, but this seems increasingly unlikely. So Post's tag system probably does not simulate a Turing machine.

The question as to which $\sigma_{n}$ die and which cycle remains a mystery. Up to $n=6075$, Blomberg's results show that about one-sixth of the values of $n$ die and fivesixths cycle. The precise values can be found in A291792. It would be nice to understand this sequence better.

\section{Coordination Sequences}

This final section is concerned with coordination sequences, which arise in crystallography and in studying tiling problems, have beautiful illustrations, and lead to many unsolved mathematical questions.

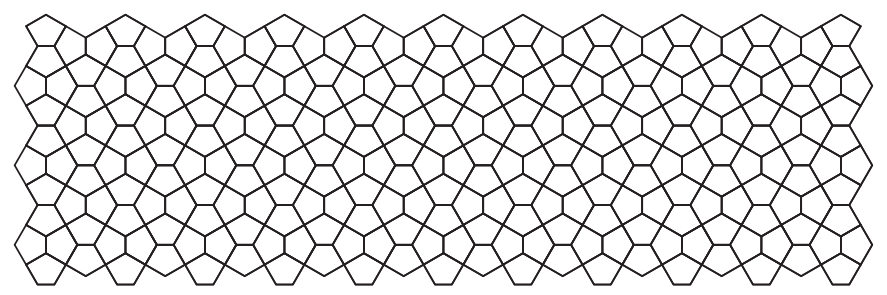

Figure 17. A portion of the Cairo tiling.

The "Cairo" tiling, so called because it is said to be used on many streets in that city, is shown in Figure 17. Let $G$ denote the corresponding infinite graph (with vertices for points where three or more tiles meet, and edges between two vertices where two tiles meet). The figure is also a picture of the graph.

The distance between vertices $P, Q \in G$ is defined to be the number of edges in the shortest path joining them. The coordination sequence of $G$ with respect to a vertex $P \in G$ is then the sequence $a(n)(n \geq 0)$ giving the number of vertices $Q$ at distance $n$ from $P$. Coordination sequences have been studied by crystallographers for many years [5].

The graph of the Cairo tiling has two kinds of vertices, trivalent (where three edges meet) and tetravalent. As can be seen from Figure 17, the coordination sequence with respect to a tetravalent vertex begins $1,4,8,12,16,20,24, \ldots$, which appears to be the same as the coordination sequence A008574 for a vertex in the familiar square grid. This observation seemed to be new. Chaim Goodman-Strauss and I thought that such a simple fact should have a simple proof, and we developed an elementary "coloring book" procedure [4] which not only proved this result but also established a number of conjectured formulas for coordination sequences of other tilings mentioned in entries in the OEIS. The "coloring book" drawing of the Cairo graph centered at a tetravalent vertex is shown in Figure 18. This coloring makes it easy to prove that the coordination sequence is given by $a(n)=4 n$ for $n \geq 1$ (see [4] for details). 


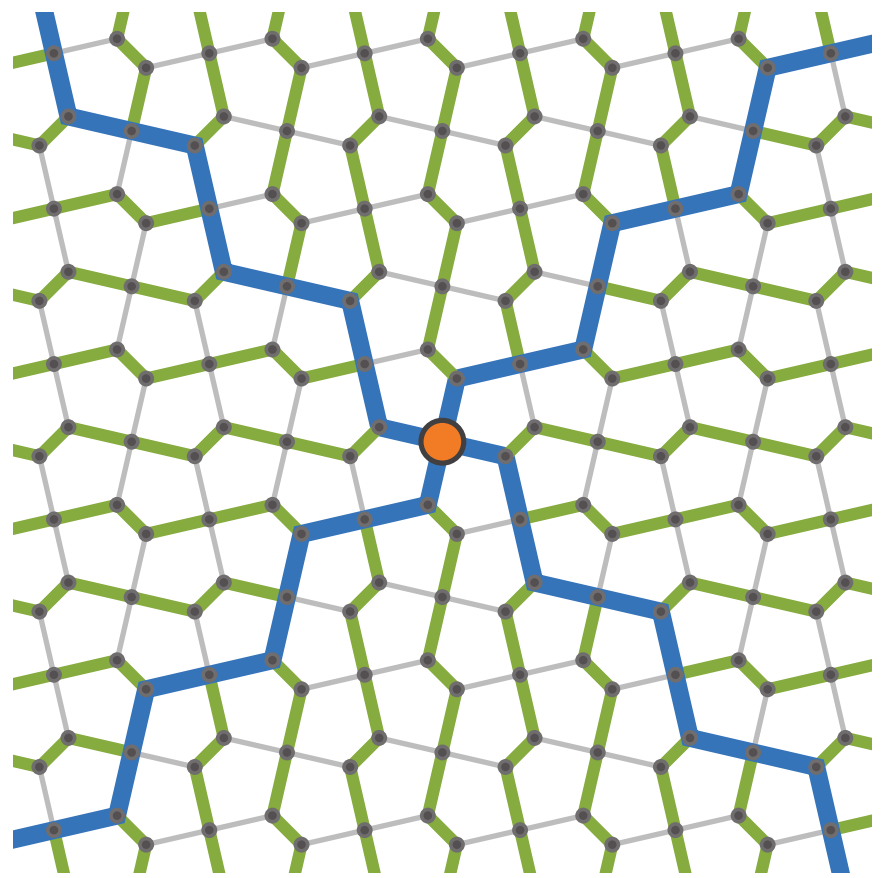

Figure 18. The "coloring book" method applied to a tetravalent vertex (the red dot) in the Cairo tiling, used to prove that the coordination sequence is the same as that for the square grid.

For a trivalent vertex in the Cairo tiling, the coordination sequence is

$$
\begin{aligned}
& 1,3,8,12,15,20,25,28, 31,36, \\
& 41,44,47,52,57,60,63,68, \ldots
\end{aligned}
$$

(this is now A296368), and we [4] show that for $n \geq 3$, $a(n)=4 n$ if $n$ is odd, $4 n-1$ if $n \equiv 0(\bmod 4)$, and $4 n+1$ if $n \equiv 2(\bmod 4)$.

One can similarly define coordination sequences for other two- and higher-dimensional structures, and the OEIS presently contains over 7000 such sequences (mostly without formulas). Many more could be added. There are many excellent websites with lists of tilings and crystals. Brian Galebach's website ${ }^{12}$ is especially important, as it includes pictures of all " $k$-uniform" tilings with $k \leq 6$, with over 1000 tilings. Darrah Chavey's article [2] and the Michael Hartley and Printable Paper websites ${ }^{13}$ have many further pictures, and the RCSR and ToposPro databases ${ }^{14}$ have thousands more.

Only last week (on May 4, 2018), Rémy Sigrist investigated the Ammann-Beenker (or “octagonal”) tiling shown in Figure 19, an aperiodic tiling with eight-fold rotational symmetry about the central point.

\footnotetext{
12 probabilitysports.com/tilings.html.

13 www.dr-mikes-math-games-for-kids.com/archimedean -graph-paper.htm1, https://www.printablepaper.net /category/graph.

${ }^{14}$ rcsr. net, topospro.com.
}

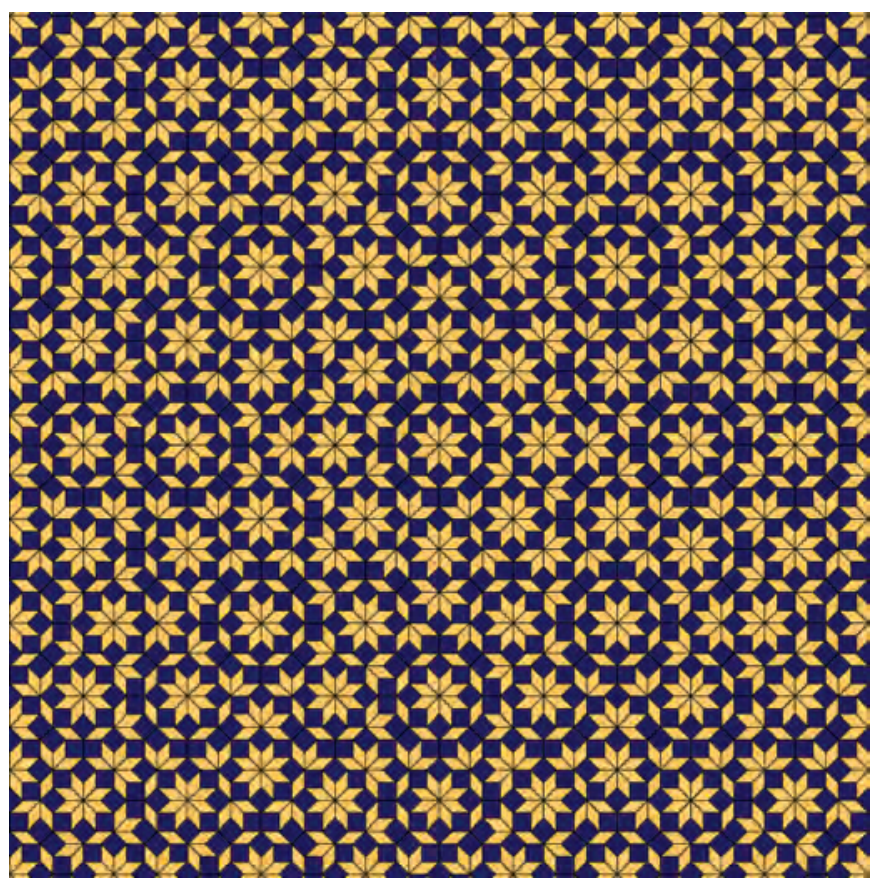

Figure 19. Ammann-Beenker (or "octagonal”) tiling.

Sigrist determined the initial terms of the coordination sequence with respect to the central vertex (A303981):

(6) $1,8,16,32,32,40,48,72,64,96,80$,

$104,112,112,128,152, \ldots$

Figure 20 shows the vertices at distances $0,1,2, \ldots, 6$ from the center.

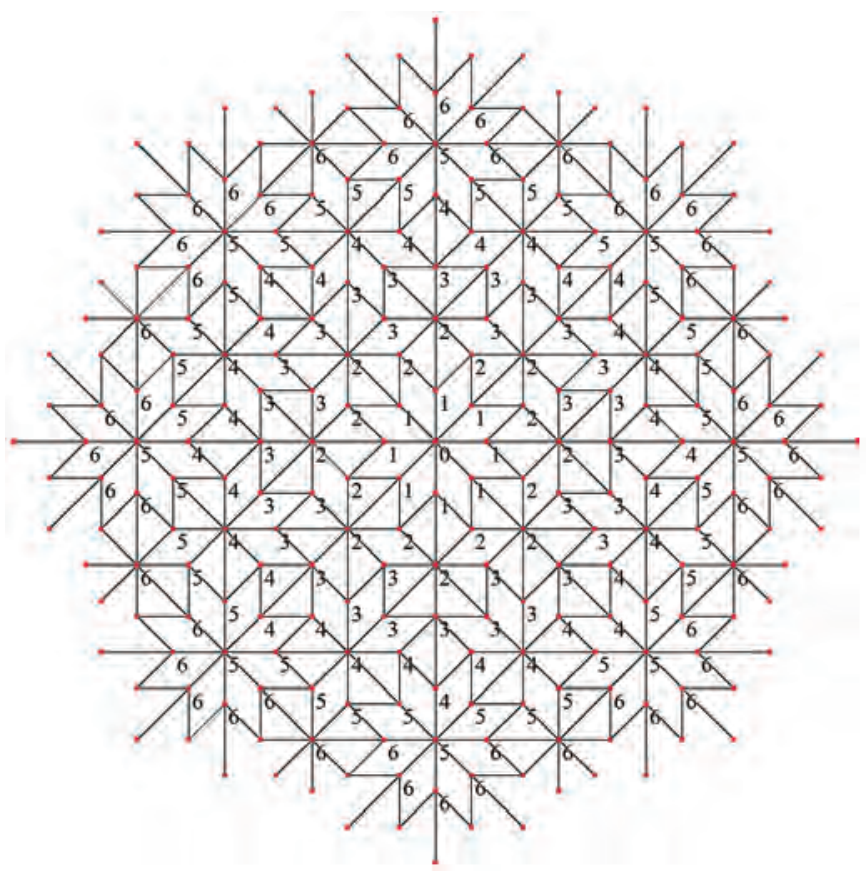

Figure 20. Illustrating the coordination sequence for the Ammann-Beenker tiling, showing the vertices at distances 0 though 6 from the central vertex. 
No formula or growth estimate is presently known for this sequence. However, earlier this year Anton Shutov and Andrey Maleev determined the asymptotic behavior of the coordination sequence (A302176) with respect to a vertex with five-fold rotational symmetry in a certain Penrose tiling. So we end with a question: Can the ShutovMaleev approach be used to find the asymptotic growth of (6)? Of course an explicit formula would be even nicer.

\section{Acknowledgments}

Now that the OEIS is a wiki, many volunteer editors help maintain it. ${ }^{15}$ Besides the OEIS contributors already mentioned in this article, I would like to thank Jörg Arndt, Michael De Vlieger, Charles Greathouse IV, Alois P. Heinz, Michel Marcus, Richard J. Mathar, Don Reble, Jon E. Schoenfield, and Allan C. Wechsler for their help. (Many other names could be added to this list.)

\section{References}

[1] D. L. Applegate, H. Havermann, B. Selcoe, V. Shevelev, N. J. A. SloAne, and R. Zumkeller, The Yellowstone Permutation, J. Integer Seqs., 18 (2015), \#15.6.7. MR3360900

[2] D. CHAVEY, Tilings by regular polygons II: A catalog of tilings, Computers \& Mathematics with Applications, 17:1-3 (1989), 147-165. MR994197

[3] P. ERDŐs, R. FREUD, and N. HegYvÁRI, Arithmetical properties of permutations of integers, Acta Math. Hungar., 41 (1983), 169-176. MR704537

[4] C. Goodman-Strauss and N. J. A. Sloane, A coloring book approach to finding coordination sequences, Acta. Cryst. A, to appear: arXiv:1803.08530, 2018.

[5] M. O'KeEFE and B. G. HYDE, Plane nets in crystal chemistry, Phil. Trans. Royal Soc. London, Series A, Mathematical and Physical Sciences, 295:1417 (1980), 553-618. MR569155

[6] P. VANDERLIND, R. K. GUY, and L. C. LARSON, The Inquisitive Problem Solver, MAA Press, 2002. MR1917371

\section{Image Credits}

Figures 1-5, 10, 11, 13, 15 courtesy of Michael De Vlieger (who also designed the "Peace to the Max" T-shirt in Figure 3).

Figure 6 courtesy of Jessica Gonzalez.

Figures 7-9 courtesy of Jonathan Wild.

Figure 12 courtesy of Hans Havermann.

Figure 14 courtesy of Kerry Mitchell.

Figure 16 courtesy of Lars Blomberg.

Figures 17 and 18 courtesy of Chaim Goodman-Strauss.

Figure 19 from The Tilings Encyclopedia, https://tilings . math . uni-

bi e1efe1d. de. Used under the Creative Commons Attribution-

NonCommercial-ShareAlike 2.0 Generic License.

Figure 20 courtesy of Rémy Sigrist.

Author photo courtesy of Susanna Stevens Cuyler.

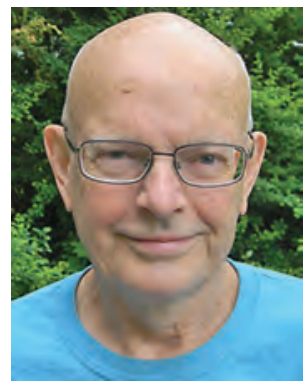

Neil J. A. Sloane

\section{ABOUT THE AUTHOR}

Neil J. A. Sloane started collecting sequences in 1964. Every advance in computing since then has made the task easier; an apt subtitle to this article could be "From punched cards to wiki in fiftyfour years." The Unix operating system was the greatest breakthrough of all, especially grep, the programmable editor ex, and pipes.

\footnotetext{
${ }^{15}$ And more are needed-if interested, please contact the author.
} 


\section{CBMS SURVEY 2015 NOW AVAILABLE}

Statistical Abstract of Undergraduate Programs in the Mathematical Sciences in the United States

by Richelle Blair, Ellen E. Kirkman, and James W. Maxwell

Every five years the Conference Board of Mathematical Sciences (CBMS) sponsors a national survey of undergraduate mathematical and statistical sciences in the nation's four-year and two-year universities and colleges. In this latest survey, learn more about

- Enrollments

- Curriculum

- Bachelor's degrees awarded

- Course availability

- Faculty demographics

- Special one-time topics:

$\triangleright$ departments

teacher certification and licensure

distance learning and other changes in pedagog and curriculum

dual enrollments and AP credit

$\triangleright$ students

participation in research, internships, or consulting majors' post-graduation plans

Survey results can be used by departments and at the state and national level to inform strategy and support funding for programs in mathematics, science, and technology.

Access this important volume at ams.org/cbms2015 or request a hard copy by emailing cbms-survey@ams.org.
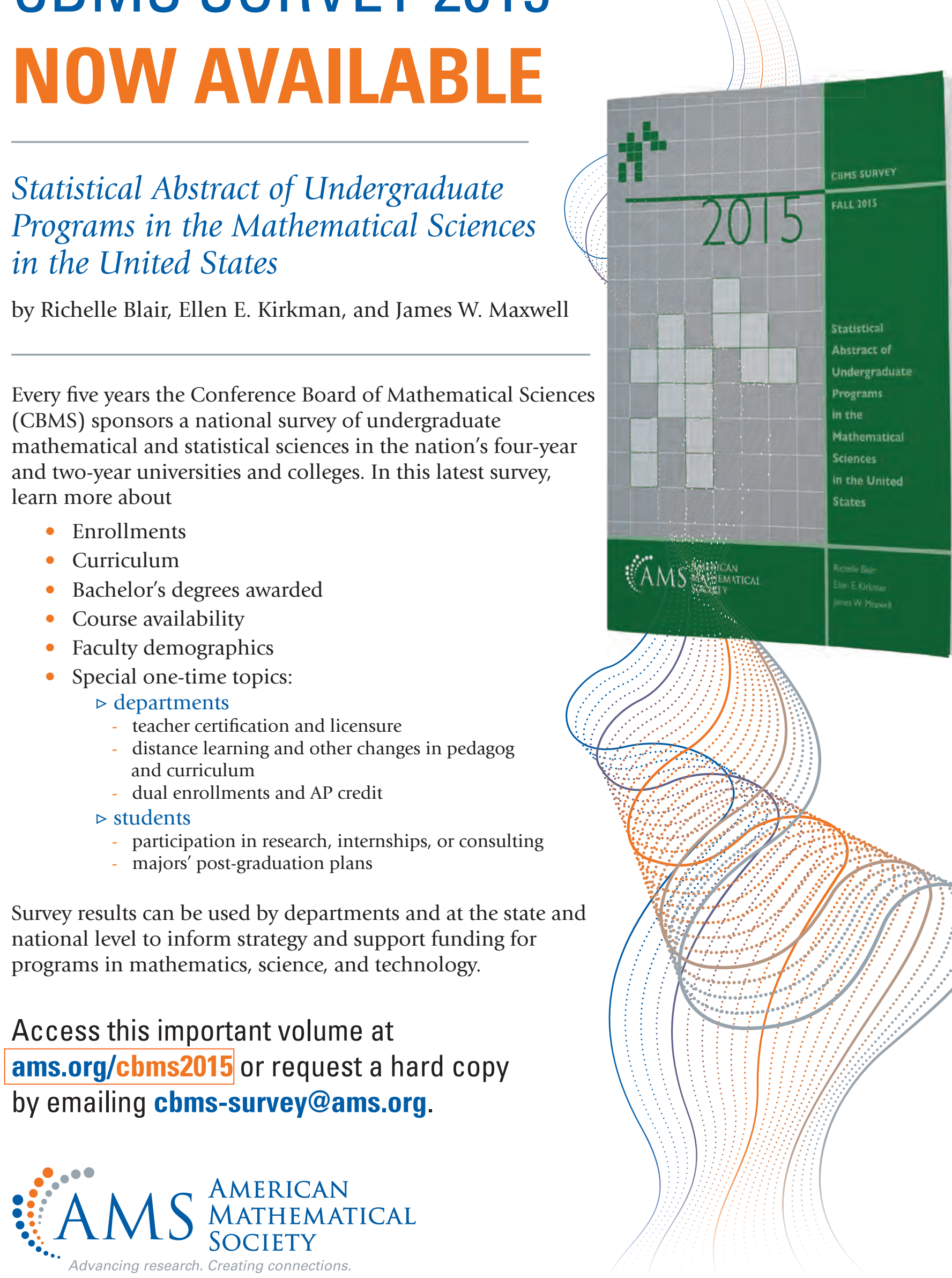


\section{AMS FALL WESTERN SECTIONAL}
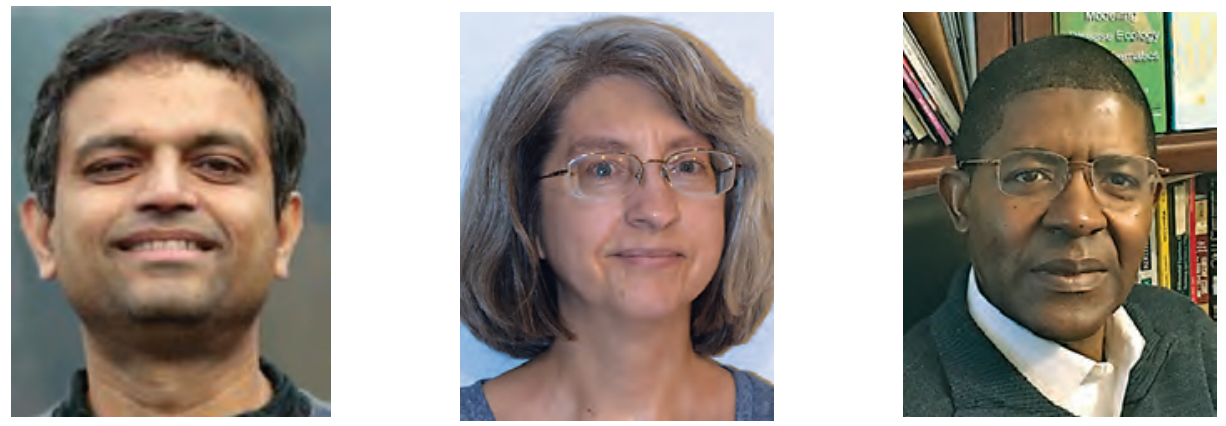

From left to right: Srikanth B. Iyengar, Sarah Witherspoon, and Abdul-Aziz Yakubu.

In this sampler, the speakers below have kindly provided introductions to their Invited Addresses for the AMS Fall Western Sectional taking place October 27-28 at San Francisco State University.

Finite Free Complexes over Polynomial Rings Srikanth B. Iyengar (University of Utah) page 1077

Derivatives, Derivations, and Hochschild Cohomology

Sarah Witherspoon (Texas A\&M University) page 1078

Population Cycles in Discrete-time Infectious Disease Models Abdul-Aziz Yakubu (Howard University) page 1079

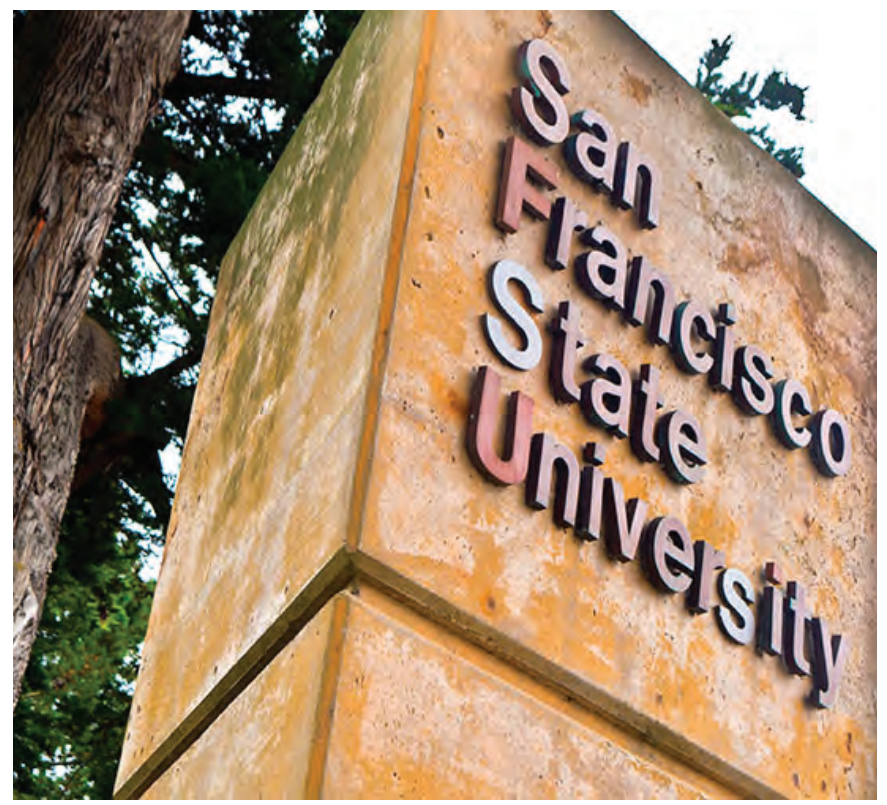

\section{EDITOR'S NOTE.}

Although we have no samplers from the AMS Central Sectional at University of Michigan, October 20-21, it also looks like a great meeting: www. ams . org/meetings/

sectiona1/2259_program.htm 1

For permission to reprint this article, please contact:

reprint-permission@ams.org.

DOI: http://dx.doi.org/10.1090/noti1709 


\section{AMS FALL WESTERN SECTIONAL}

\section{Srikanth B. Iyengar}

\section{Finite Free Complexes over Polynomial Rings}

The story I want to present begins with Hilbert. It concerns linear operators acting on a vector space $V$ over a field $k$. The action of commuting linear operators $t_{1}, \ldots, t_{d}$ on $V$ can be codified into an action of the polynomial ring $S:=k\left[x_{1}, \ldots, x_{d}\right]$ on $V$, with each $x_{i}$ acting via $t_{i}$. Said otherwise, $V$ is an $S$-module. A simple example is $V=S$, with each $x_{i}$ acting by multiplication. The free module (of rank $n$ ) is the $n$-fold direct sum, $S^{n}$, with the natural action. These are called free modules because the action is as free as possible, in that they induce no relations on the $x_{i}$. Not every module is free: consider $k$ with each $x_{i}$ acting as zero. Hilbert's Syzygy Theorem states that any $S$-module $V$ can be resolved by free $S$-modules, in the sense that there is a diagram of maps

(†) $0 \longrightarrow S^{\beta_{n}} \stackrel{\partial_{n}}{\longrightarrow} S^{\beta_{n-1}} \stackrel{\partial_{n-1}}{\longrightarrow} \cdots \stackrel{\partial_{2}}{\longrightarrow} S^{\beta_{1}} \stackrel{\partial_{1}}{\longrightarrow} S^{\beta_{0}} \longrightarrow 0$, where $\partial_{i}$ is compatible with the $S$-action, $\operatorname{Kernel}\left(\partial_{i}\right)=$ Image $\left(\partial_{i+1}\right)$ for each $i \geq 1$, and Cokernel $\left(\partial_{1}\right)=V$. The remarkable point is that only finitely many free modules are involved; indeed Hilbert proved that there exists a resolution with $n \leq d$. The module Image $\left(\partial_{i}\right)$ is called an $i$ th syzygy of $V$, so the theorem says that $V$ has a syzygy that is free. The integers $\beta_{i}$ are called the Betti numbers of $V$; these are algebraic analogues of Betti numbers in topology, and they are just as interesting as their topological counterparts. The prototypical example is a resolution of $k$ called the Koszul complex. It appears in essentially every article I have written; this one need not be an exception, so here it is for $d=3$ :

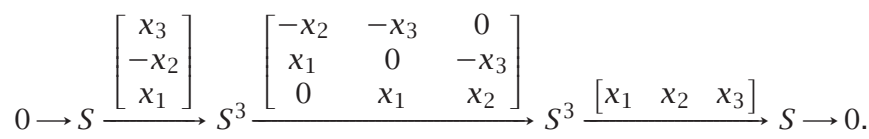

I am viewing the elements of the various free modules as column vectors. You can guess what the Koszul resolution of $k$ is for a general $d$; the Betti numbers satisfy $\beta_{i}=\left(\begin{array}{l}d \\ i\end{array}\right)$.

In the early 1970s Buchsbaum and Eisenbud conjectured that whenever the $k$-vector space $V$ is finite-dimensional, its Betti numbers satisfy $\beta_{i} \geq\left(\begin{array}{l}d \\ i\end{array}\right)$. This conjecture remains open. It implies the following.

Total Rank Conjecture. $\sum_{i=0}^{n} \beta_{i} \geq 2^{d}$.

This inequality was conjectured by Horrocks and also by Avramov. It was settled recently by Mark Walker whenever the characteristic of $k$ is not two using a beautiful argument involving Adams operations on complexes.

Srikanth B. Iyengar is professor of mathematics at the University of Utah. His email address is iyengar@math. utah. edu.

For permission to reprint this article, please contact:

reprint-permission@ams.org.

DOI: http://dx.doi.org/10.1090/noti1728
A complex is a diagram $F$ of the form $(\dagger)$ where $\operatorname{Kernel}\left(\partial_{i}\right)$ contains Image $\left(\partial_{i+1}\right)$ for each $i$; in the cases of interest to us the corresponding quotient vector space, called the homology of $F$ in degree $i$, is finite-dimensional. Resolutions of finite-dimensional modules are examples of such complexes, but many natural constructs lead to the more general kind. Experience had led us to expect that properties of resolutions of finite-dimensional modules would carry over to such $F$. In particular, it had been conjectured (by me anyway) that the Total Rank Conjecture holds also for such complexes.

Walker visited me last year, and within a few minutes of our meeting (the date, April 27, is unforgettable) we realized that between what he knew and what I had learnt recently about Lefschetz elements in exterior algebras-from a collaboration with Conca and Herbigwe could construct counterexamples to the Total Rank Conjecture for complexes. Subsequently it dawned on us that the same simple idea yields counterexamples to several other conjectures, including one due to Avramov and Buchweitz about Betti numbers of modules over complete intersection rings.

Lower bounds (almost all conjectural) similar to the Total Rank Conjecture appear also in the context of group actions on spaces. Notable among these is a conjecture of Carlsson for spaces admitting a free action of $(\mathbb{Z} / p \mathbb{Z})^{r}$, the elementary abelian $p$-group of rank $r$, where $p$ is a prime number, and one due to Halperin about spaces that admit a free action of the torus, $\left(S^{1}\right)^{r}$. Mark and I could also construct counterexamples to algebraic analogues of their conjectures. These examples challenge long-held intuition about finite free complexes and have sowed a sense of confusion in me. I'll try to convey some of this in my talk.

\section{Photo Credit}

Author photo by Tatjana Ruf, copyright MFO, used on the terms of the Creative Commons License Attribution-Share Alike 2.0 Germany.

\section{ABOUT THE AUTHOR}

Srikanth B. Iyengar is interested in commutative algebra in its various manifestations, and in particular in the modular representation theory of finite groups. He was awarded the Bessel Research Prize (2007) by the Humboldt Foundation, was a Simons Fellow in Mathematics (2012-13), and is a

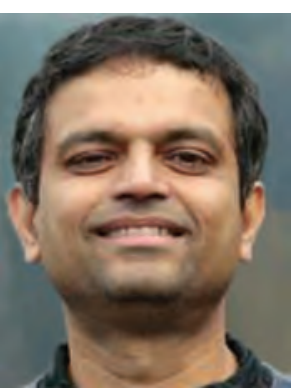

Srikanth B. Iyengar Fellow of the American Mathematical Society. 


\section{AMS FALL WESTERN SECTIONAL}

\section{Sarah Witherspoon}

\section{Derivatives, Derivations, and Hochschild Cohomology}

In the 1940s, inspired by homological methods in topology, Hochschild, Eilenberg, and Mac Lane introduced them in algebra. One outcome was Hochschild cohomology. It encodes essential information about rings, topological spaces, and categories. It has many applications, users, and developers. For example, it encodes possible deformations of an algebra, it measures coarse properties of representations via support varieties, and it determines geometric properties such as smoothness in both commutative and noncommutative settings. Yet Hochschild cohomology also has some remaining mysteries. These are the focus of much current research and of my talk.

This story begins with the Leibniz rule for differentiation of functions $f$ and $g$ on the real line:

$$
\frac{d}{d x}(f g)=\frac{d}{d x}(f) g+f \frac{d}{d x}(g) .
$$

Generalize this rule to elements of rings $R$ that are also vector spaces over fields such as $\mathbb{C}$ or $\mathbb{R}$ : A linear function $\delta$ from $R$ to $R$ is called a derivation if

$$
\delta(f g)=\delta(f) g+f \delta(g)
$$

for all $f$ and $g$ in $R$. Fundamental examples are indeed given by differentiation on rings of functions, such as polynomial rings or functions on a manifold.

The set of all derivations on $R$ forms a vector space. This vector space is not closed under composition of functions. However it is closed under a related binary operation: If $\delta$ and $\delta^{\prime}$ are two derivations on $R$, then so is $\left[\delta, \delta^{\prime}\right]$, defined as

$$
\left[\delta, \delta^{\prime}\right]:=\delta \circ \delta^{\prime}-\delta^{\prime} \circ \delta .
$$

The operation [ , ] is called a commutator or Lie bracket, and the vector space of derivations together with this bracket operation is called a Lie algebra. Vector fields on manifolds are derivations on their rings of functions, forming central examples of such Lie algebras.

More generally, consider multilinear functions from $R \times \cdots \times R$ to $R$. What is a suitable analogue of derivation? Hochschild's definition of cohomology for rings answers this question via multilinear analogues of the Leibniz rule. Hochschild cohomology spaces consist of multilinear functions obeying these rules.

Is there an analogue, for multilinear functions, of the Lie bracket on derivations? Yes! In the 1960s Gerstenhaber introduced a bilinear operation on multilinear functions, and thus on Hochschild cohomology, that is now called

Sarah Witherspoon is professor of mathematics at Texas A\&M University. Her email address is sjw@math. tamu.edu.

For permission to reprint this article, please contact:

reprint-permission@ams.org.

DOI: http://dx.doi.org/10.1090/noti1726

the Gerstenhaber bracket. Together with additional algebraic structure and fortuitous properties, Hochschild cohomology spaces are important algebraic invariants for rings.

Since its introduction by Hochschild and its further development by Gerstenhaber and others, Hochschild cohomology has found applications in many settings, for example in algebraic deformation theory, representation theory, and noncommutative geometry. For some of these applications, a deep understanding of the Gerstenhaber bracket is required. A view of Hochschild cohomology as a certain Ext group has long promised insight that is realized in recent work. In my talk I will survey some of this work as well as give an overview of Hochschild cohomology and its place in algebra.

\section{Photo Credit}

Author photo courtesy of Sarah Witherspoon.

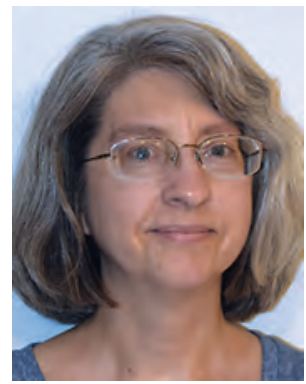

Sarah Witherspoon

\section{ABOUT THE AUTHOR}

Sarah Witherspoon's research is in noncommutative and homological algebra. She is a Fellow of the American Mathematical Society. 


\section{AMS FALL WESTERN SECTIONAL}

\section{Abdul-Aziz Yakubu}

\section{Population Cycles in Discrete-Time Infec- tious Disease Models}

ABSTRACT. The standard next generation matrix approach for calculating the basic reproduction number, an important parameter in understanding the evolution and prevention of infections, suffers from some assumptions that are not always met in real-world systems. In this talk, we explore extensions through two discrete-time infectious disease models, a SIR and an ISA $v$ model, and the different behaviors that arise.

My talk will focus on infectious disease transmission dynamics in periodic population environments. In infectious disease epidemiology, the basic reproduction number, denoted by $\mathcal{R}_{0}$, is an important threshold parameter that provides insight when designing prevention and control strategies for established infections. $\mathcal{R}_{0}$ is the average number of secondary cases produced by an infectious individual introduced into a population of susceptible individuals.

The next generation matrix (NGM) approach for defining and calculating $\mathcal{R}_{0}$ divides the population into compartments, some of them infectious. It provides an easy way to compute $\mathcal{R}_{0}$ from epidemiological reasoning and basic infectious disease model ingredients. However, for autonomous infectious disease models (without seasonality or periodicity), the NGM approach assumes the existence of a locally asymptotically stable disease-free equilibrium in the demographic equation. That is, in the absence of the disease, the population is assumed to be at a static steady state. Populations do not grow indefinitely over time, and density dependence or other factors tend to drive populations toward their carrying capacity. However, it is possible for populations to experience some fluctuations around the carrying capacity, a situation that the existing NGM approach does not address. The population fluctuations can be periodic (cyclic) or erratic (chaotic). For example, such prominent and persistent cyclic fluctuations have been observed in the abundance of some sockeye salmon populations [2].

In a recent paper, van den Driessche and Yakubu [4] used a general autonomous discrete-time epidemic model to extend the NGM approach for calculating $\mathcal{R}_{0}$ to account for period $k$ population cycles in the disease-free system, where $k \geq 1$. $\mathcal{R}_{0}<1$ implies the local asymptotic stability of the periodic disease-free population cycle and the disease goes extinct, whereas $\mathcal{R}_{0}>1$ implies the instability of the periodic disease-free cycle and the

Abdul-Aziz Yakubu is professor of mathematics at Howard University. His email address is ayakubu@howard. edu.

For permission to reprint this article, please contact: reprint-permission@ams.org.

DOI: http://dx.doi.org/10.1090/noti1727 disease persists in the population. When $\mathcal{R}_{0}<1$ and the demographic dynamics is asymptotically constant or under geometric growth (nonoscillatory, $k=1$ ), it is possible for the disease-free equilibrium point (fixed point) to be globally asymptotically stable [3]. In 2002, Elaydi and Yakubu ([1, Theorem 3]) showed that it is not possible for a disease-free population cycle with period $k \geq 2$ to be globally asymptotically stable in the "smooth" autonomous discrete-time epidemic model. In the presentation, I will apply the extended NGM approach for calculating $\mathcal{R}_{0}$ to two discrete-time infectious disease models with Ricker recruitment functions. The first model is a Susceptible-Infectious-Recovered (SIR) model with and without vaccination, and the second one is an Infectious Salmon Anemia Virus (ISAv) model [4].

The Ricker model was first used by Ricker in 1954 to study population cycles in fish. To illustrate densitydependent population cycles in a demographic equation (in the absence of a disease), in Figure 1 we consider the discrete-time demographic equation with a Ricker recruitment,

$$
S_{t+1}=f\left(S_{t}\right)+(1-d) S_{t}, \quad t \in\{0,1,2, \ldots\},
$$

with $S_{t}$ the population size of the susceptible individuals, Ricker recruitment function

$$
f\left(S_{t}\right)=r S_{t} e^{-b S_{t}},
$$

$r>0$ the intrinsic growth rate, and $b>0$ a scaling parameter. During each unit time interval, $d \in(0,1)$ is the probability of natural death for each individual, and $(1-d)$ is the probability of staying alive. Using knowledge of the population size of the susceptible individuals, $S_{t}$, at time $t$, model (1) predicts $S_{t+1}$. The model's unit of time depends on the specific application. For example, the unit of time could be a convenient time for a follow-up census.

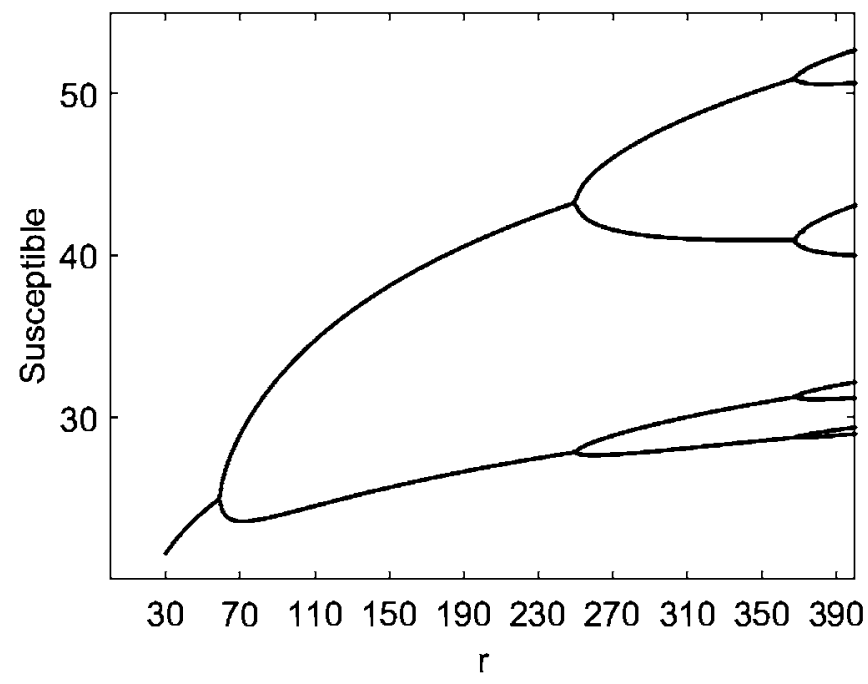

Figure 1. As the intrinsic growth rate $r$ increases, the number $S$ of susceptibles exhibits period-doubling bifurcations en route to chaos. 


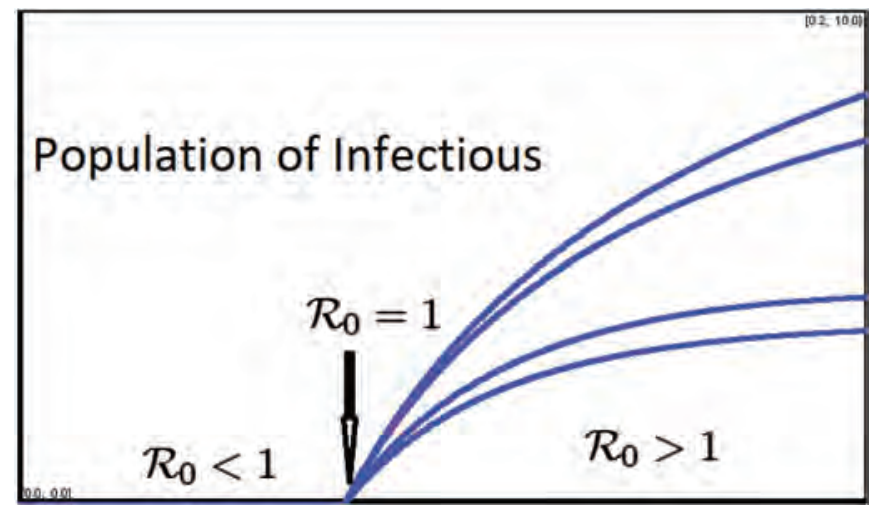

Figure 2. In this bifurcation diagram for the SIR model, as $\mathcal{R}_{0}$ increases from values less than 1 to values greater than 1 , the dynamics change from disease extinction to disease persistence on a locally asymptotically stable period 4 population cycle.

In model (1), the population goes extinct when $\mathcal{R}_{d}=$ $\frac{r}{d}<1$. However, when $\mathcal{R}_{d}>1$ the disease-free population persists on a fixed-point or cyclic or chaotic attractor. When $1<\mathcal{R}_{d}<e^{\frac{2}{d}}$, the disease-free system persists on the fixed-point attractor, $Z_{\infty}=\frac{\ln \mathcal{R}_{d}}{b}$. When $\mathcal{R}_{d}>e^{\frac{2}{a}}$, the disease-free equation (1) is known to undergo perioddoubling bifurcations en route to chaos as in Figure 1.

In general, the birth or recruitment function $f$ of model (1) is a nonlinear function that is capable of exhibiting cyclic or chaotic dynamics. An epidemic process is built on top of the demographic pattern generated by $f$. To guarantee control over the disease-free dynamics (no matter how complex they are) we assume that the disease does not affect $f$ in a significant way. This approach to model construction is quite common for continuous-time epidemic processes but less common for discrete-time epidemic models ([3], [4]).

We construct the SIR and ISA $v$ discrete-time infectious disease models on top of model (1). SIR models have been used to study a variety of diseases in humans and animals, while ISA $v$ models have been primarily used to study infectious salmon anemia, a finfish disease caused by a virus that belongs to a family of viruses called Orthomyxoviridae. ISA $v$ has caused significant mortality among salmon farms in northern Europe, Canada, Maine, and Chile. Our SIR and ISA $v$ models share a common demographic equation with a Ricker recruitment function. In the presentation, I will use the extended NGM approach to compute $\mathcal{R}_{0}$ for both models. In the SIR model, the dynamics of the Ricker demographic equation in the absence of the disease is qualitatively equivalent to the dynamics of the total population, $S+I+R$. However, in the ISA $v$ model, the dynamics of the salmon population equation, in the absence of the disease, is qualitatively different from that of the total salmon population, $S+I$. When $\mathcal{R}_{0}>1$, I will show that the period of the disease-free

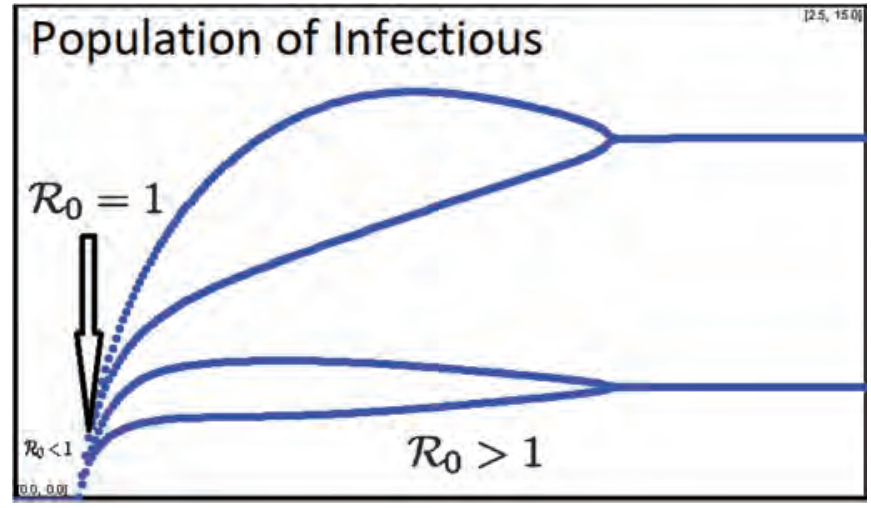

Figure 3. In this bifuration diagram for the ISAv model, as $\mathcal{R}_{0}$ increases from values less than 1 to values greater than 1 , the dynamics change from disease extinction to disease persistence on a period 4 or 2 population cycle.

susceptible population cycle in the SIR model determines the period of the infectious population cycle (see Figure 2).

Unlike the SIR model, in the ISA $v$ model, the period of the infectious salmon population can be different from that of the cyclic disease-free salmon population. In particular, I will illustrate that the ISA $v$ disease is capable of stabilizing population fluctuations in the salmon population (see Figure 3). Figure 3 shows an ISA $v$ disease-induced period-doubling reversal bifurcation, where the diseasefree salmon population is on a period 4 population cycle. The extended NGM approach accounts for periodic population cycles, and the resulting $\mathcal{R}_{0}$ computed can be used to guide prevention and control strategies in additional infectious disease environments.

\section{References}

[1] S. ELAYDI and A.-A. YAKUBU, Global stability of cycles: LotkaVolterra competition model with stocking, J. Diff. Equations Appl. 8(6) (2002), 537-549. MR1903949

[2] D. A. LeVY and C. C. Wood, Review of proposed mechanisms for sockeye salmon population cycles in the Fraser River, Bull. Math. Biol. 54 (2-3) (1992), 241-261.

[3] P. VAN DEN DRIESSChe and A -A. YAKUbU, Disease extinction versus persistence in discrete-time epidemic models, Bull. Math. Biol. (2018) https://doi .org/10 .1007/s11538-0180426-2

[4] P. VAN DEN DRIESSChe and A.-A. YAKUbU, Demographic population cycles and $\mathcal{R}_{0}$ in discrete-time epidemic models, preprint (2018) (submitted). 


\section{AMS FALL WESTERN SECTIONAL}

\section{Image Credits}

All images courtesy of Abdul-Aziz Yakubu.

\section{ABOUT THE AUTHOR}

Abdul-Aziz Yakubu's research interests include mathematical biology and dynamical systems. He coorganized several international mathematical biology workshops and conferences in Africa and was visiting professor at Botswana International University of Science and Technology, Cornell University, North East Fisheries Science Center, and The Ohio State Univer-

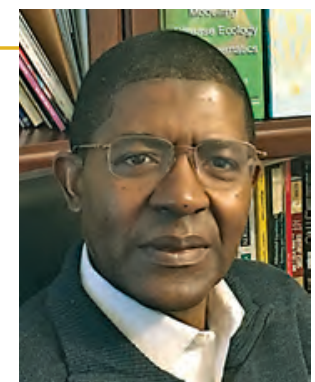

Abdul-Aziz Yakubu sity. 


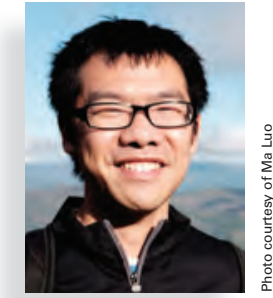

Ma Luo

Favorite mathematics book:

A Scrapbook of Complex

CurveTheory

\section{Rebecca R.G.}

Best piece of advice received in graduate school: You will often feel like your work is insignificant or trivial, because it seems obvious to you. Get a second opinionsomeone on the outside may know that it's difficult and worthwhile.

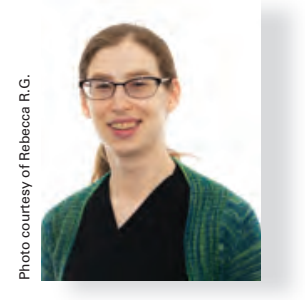

Matthew Junge What do you miss most about graduate school?

The camaraderie and commiseration.

\section{Jaclyn Lang}

Best piece of advice received in graduate school: You have to celebrate all of your accomplishments as they happen; no one will do it for you, and it's important.

\section{Andrei Prokhorov}

Best piece of advice received in graduate school: The moment right after you solved the problem is the most

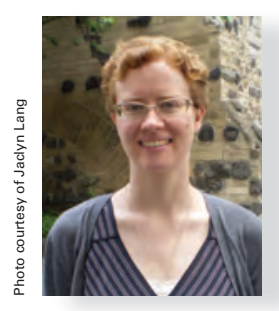
enjoyable, and you need to appreciate it.

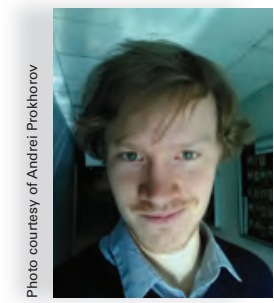

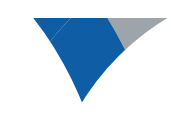

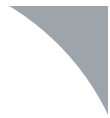

\section{THE NEXT GENERATION DF MATHEMATICS}

Early Career AMS Members Take a

Moment to Share a Little About Themselves:

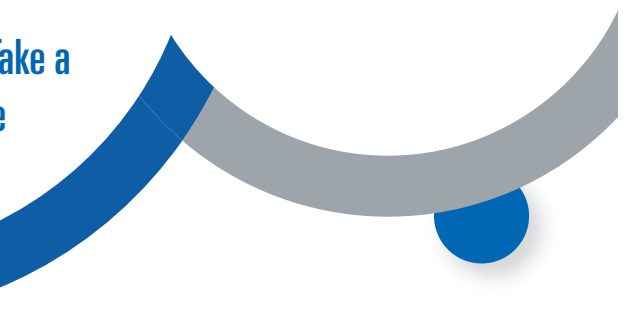

\section{Roy Araiza}

25 years from now: I am a professor at a university that has a strong operator algebras group, and my work has been to apply operator space theory techniques to some of the largest problems in the field. Aside from my mathematical accomplishments, I have been an advocate for the involvement of underrepresented groups in mathematics. Being a Latino in mathematics I believe it is not only my job to celebrate underrepresented groups in mathematics but my obligation to assure that they will not be a minority in the profession for long.

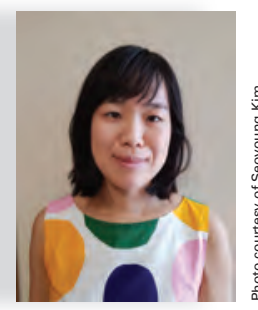

\section{Seoyoung Kim} 25 years from now: I want to be a good advisor and contributing a lot to the math community!

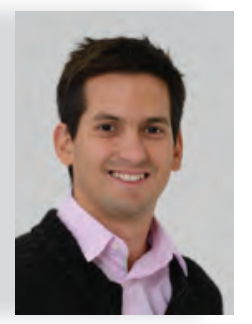

Luis Sordo Vieira

The biggest difference between being a faculty member and being a graduate student: In early graduate school, I thought of people with their PhDs as infallible, and that science was easy for them. As a postdoc, I now realize I am just as fallible as a graduate student, and that science is in fact very hard! 
Elizabeth Upton

25 years from now: In student review form: "Professor Upton has inspired

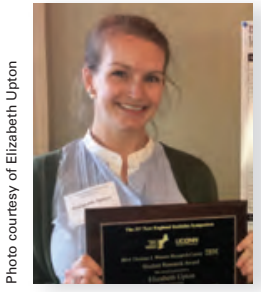

me to continuing studying statistics! She presented the material in a clear, effective and interesting way, all while underpinning the importance and power of the subject."

\section{Vanessa Rivera- Quiñnones}

Best piece of advice received in graduate

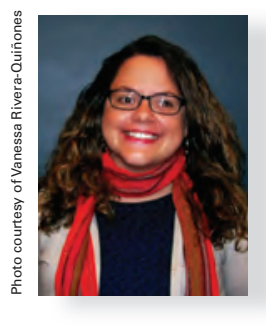

school: The best piece of advice I've received is to surround yourself with people who support you. It takes a village to get through graduate school and I've been lucky to have great mentors along the way that have supported and guided me throughout the years. From choosing an advisor, finding a great therapist, or joining a professional organization that speaks to your interests, it may look different for everyone and finding what you need is part of the journey.

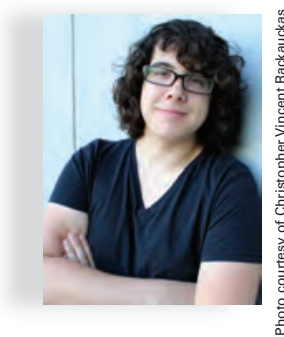
Christopher
Vincent
Rackauckas

Favorite mathematics

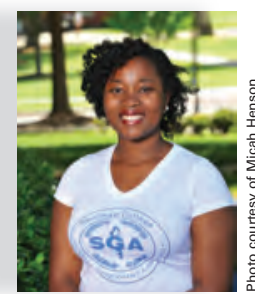
Micah

Best piece of advice received in graduate school: The best piece of advice that I have received and that is

helping me get through grad school is that my worth is not in my ability to do mathematics. It seems pretty simple, but when I am not performing as well as I think I should be, it reminds me to not get wrapped up in my failures and ultimately helps me to move on and keep working.

\section{Charles Camacho}

What is the one mathematics book you could not give up? Introduction to

Compact Riemann Surfaces and Dessins d'Enfants by Ernesto Girondo and Gabino González-Diez. This was the book that got me into research, and I carried it around in my backpack for at least two years.

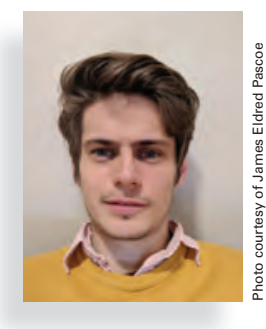

James Eldred Pascoe

\section{Best piece of advice received in graduate school: Remmel}

Reginald
"RB" McGee

Favorite course in graduate school: Complex Analysis,

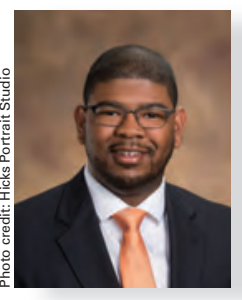

Sensing, and Wine Appreciation

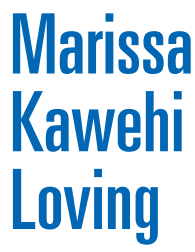

Best piece of advice received in graduate school: One of the best pieces of advice I was given in graduate school was from my undergraduate advisor who I would often talk to when math wasn't going well and I felt close to giving up on the entire grad school thing. He told me, "There are plenty of people who are ready to close doors on you. Don't do it for them." It's not easy to believe in yourself when it feels like no one else does, but sometimes you have to be your own advocate. Although it sure helps to have a group of close friends in your graduate cohort to lean on when your own strength is failing. Intro to Compressed

told me, "There is a lot of mathematics in the world, you might as well do something you like."

book: Hairer's Solving Ordinary Differential Equations 1 and 2

\section{\#AMSMember}

The AMS is dedicated to advancing research and connecting the diverse global mathematical community through our publications, meetings and conferences, MathSciNet ${ }^{\oplus}$, professional services, advocacy, and awareness programs. Early career members have many valuable benefits and services available to them as they progress in the profession. Visit the AMS Membership Headquarters today for more information about Benefits and Services: WWW.ams.org/membership. 


\section{THE GRADUATE STUDENT SECTION}

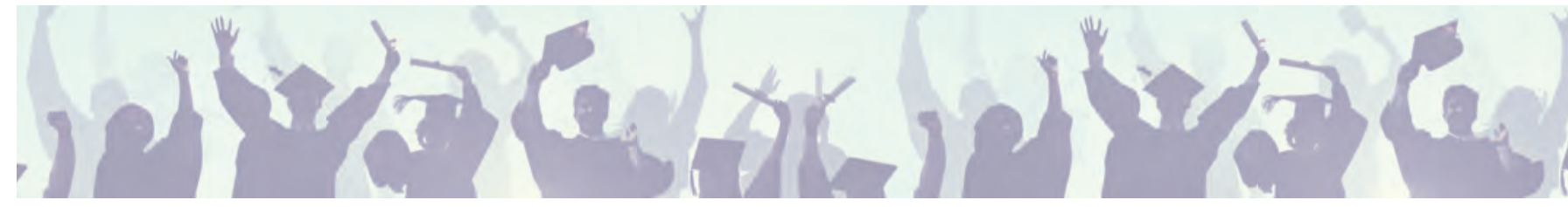

\section{Ruth Haas Interview}

\section{Conducted by Alexander Diaz-Lopez}

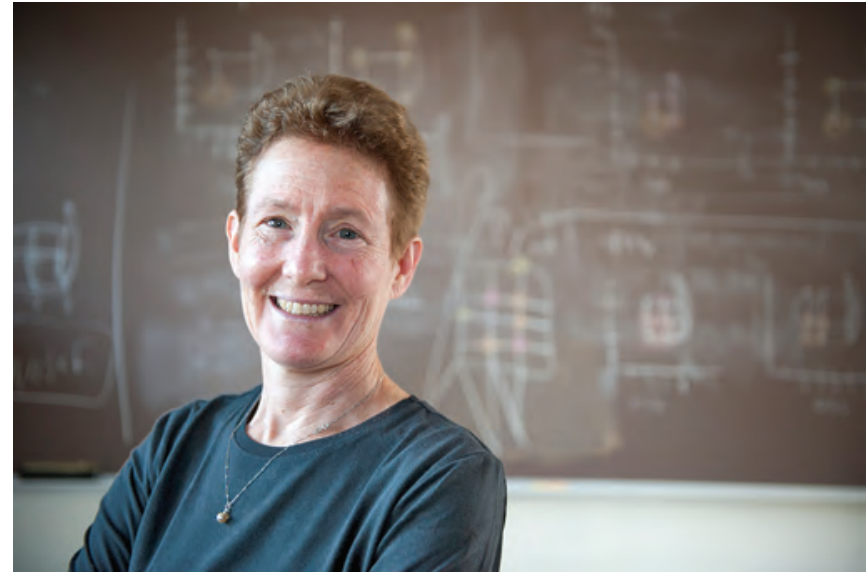

Ruth Haas, president-elect of the Association for Women in Mathematics, is professor of mathematics at the University of Hawai'i at Mānoa and Achilles Professor Emerita in Mathematics and Statistics at Smith College. At Smith, she co-founded and codirected the Center for Women in Mathematics, which received the 2011 AMS Programs That Make a Difference Award for its post-baccalaureate program. Haas received the 2015 AWM Humphreys Award for mentoring undergraduate women to continue to PhDs in the mathematical sciences. She earned her undergraduate degree at Swarthmore College and PhD at Cornell University. She has more than 30 publications in discrete mathematics, including both algebraic combinatorics and graph theory.

Diaz-Lopez: When did you know you wanted to be a mathematician?

Haas: I've always known I loved mathematics, but as a child when I said I liked math people said I should be a scientist. It was a great revelation and relief to me when I found out that math itself was a career option.

For permission to reprint this article, please contact: reprint-permission@ams.org.

DOI: http://dx.doi.org/10.1090/noti1735
Diaz-Lopez: Who encouraged or inspired you?

Haas: Early on I certainly had lots of advice to stop doing math. Some of this came from misunderstandings of what mathematics is, what mathematicians do, and certainly what a mathematician must be like (you know the stereotype). But there were people who were encouraging as well-my 7 th grade math teacher, Mrs. Ruben, and the math major who lived down the hall my freshman year, Donna Crystal-Llewellyn. For the past 30+ years my biggest support and encouragement has come from my life partner and fellow mathematician, Loek Helminck.

Diaz-Lopez: How would you describe your work to a graduate student?

Haas: Recently, I have been interested in reconfiguration problems, especially in graph theory. Given a problem with multiple feasible solutions, the reconfiguration problem asks if it is possible to get from one feasible solution to another following some reconfiguration rule. For example, can you change from one proper vertex coloring of a graph to another under the rule that you can only change the color of one vertex at a time and at each step the coloring must be proper? There are many questions to be asked about reconfiguration both about computational complexity and structure.

Diaz-Lopez: What theorem are you most proud of?

Haas: While there are theorems and proofs of which I am proud, I think I am prouder of some of the questions I've posed to others which have led them to do interesting work.

Diaz-Lopez: What advice do you have for graduate students?

Haas: I have lots of advice! To start with-get lots of advice, from lots of different people. There are many things that one might want in an ideal $\mathrm{PhD}$ advisor. Do you like the kinds of problems they work on? Do they place former students in the kinds of positions you aspire to (research postdocs, industry, small college jobs)? Do they currently have enough time/space/funding for another student? While it might be nice to have an advisor who can give you everything you want, it is really not necessary and often impossible. Your advisor doesn't have to serve 
all these roles; they just need to find other people to help you with the other aspects.

Diaz-Lopez: All mathematicians feel discouraged occasionally. How do you deal with discouragement?

Haas: That's a great question. Personally, I guess I love having the "aha" moment enough, and I have enough of them to keep me going. More generally though, I am concerned about how we as a community sometimes cultivate competition that can lead to unnecessary discouragement. Math is not a foot race where the winner and losers are clear. Not enjoying competition is not the same as not being good at math.

Diaz-Lopez: You co-founded and co-directed the Center for Women in Mathematics at Smith College, which received the AMS 2011 Programs That Make a Difference Award. Can you tell us about the Center's programs and achievements?

Haas: The Center is the first post-baccalaureate program in mathematics. The post-bac is for women who already have bachelor's degrees but are not prepared for graduate school in mathematics. They come to Smith College for a year and take advanced course work in math. Beyond the course work, the program provides a research experience and mentoring about the graduate school process. Furthermore, the students become a support network for each other as they go on to (different) graduate schools and careers. Almost all the students who have completed the program have gone on to $\mathrm{PhD}$ programs in the mathematical sciences, and they proceed to earn degrees at a higher rate than the general grad school population. I am happy to say that Smith College has agreed to partially support the program now that its NSF funding has ended.

As we all know, to be prepared for graduate school in mathematics requires a strong undergraduate mathematics major. If one has strayed from that path, it can be very difficult to re-enter the pipeline. People from groups that are underrepresented in mathematics often commit to math later, so that they are likely to benefit from a postbac program to set them up for graduate programs. The physics professional society (APS) has started an extensive post-bac program because their discipline has the same challenge. I'd like to see math find more ways to allow people to re-enter the pipeline.

Diaz-Lopez: You are president-elect of the Association for Women in Mathematics (AWM). What are your goals and objectives for AWM?

Haas: As the AWM approaches its 50th year, it is time to take stock of where we are and what we should be doing going forward. The barriers to women in mathematics are different and often more subtle than they were 50 years ago. The AWM needs to continue to find ways to encourage and promote women in mathematics at all levels. Although we have made great strides, the percentage of women PhDs in mathematics is flat or declining, and it remains the case that at every stage women drop out at a higher rate than men.

Diaz-Lopez: Any final comment or advice?

Haas: Don't forget that simply being an average mathematician is a great accomplishment. And there are lots of

\section{There are lots of places to be a mathematician.}

places to be a mathematician. In graduate school you are surrounded by high-profile faculty whose main focus is research, but the balance between teaching and research varies widely in academia, and there are jobs in industry and government labs with very different responsibilities as well.

\section{Photo Credit}

Photo of Ruth Haas courtesy of Smith College.

\section{ABOUT THE INTERVIEWER}

Alexander Diaz-Lopez, having earned his $\mathrm{PhD}$ at the University of Notre Dame, is now assistant professor at Villanova University. Diaz-Lopez was the first graduate student member of the Notices Editorial Board.

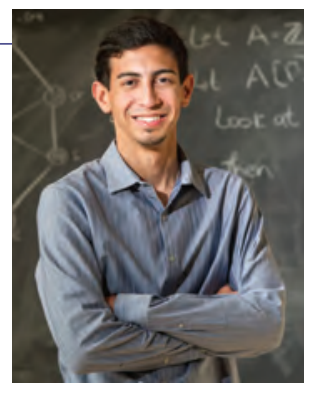

Alexander Diaz-Lopez

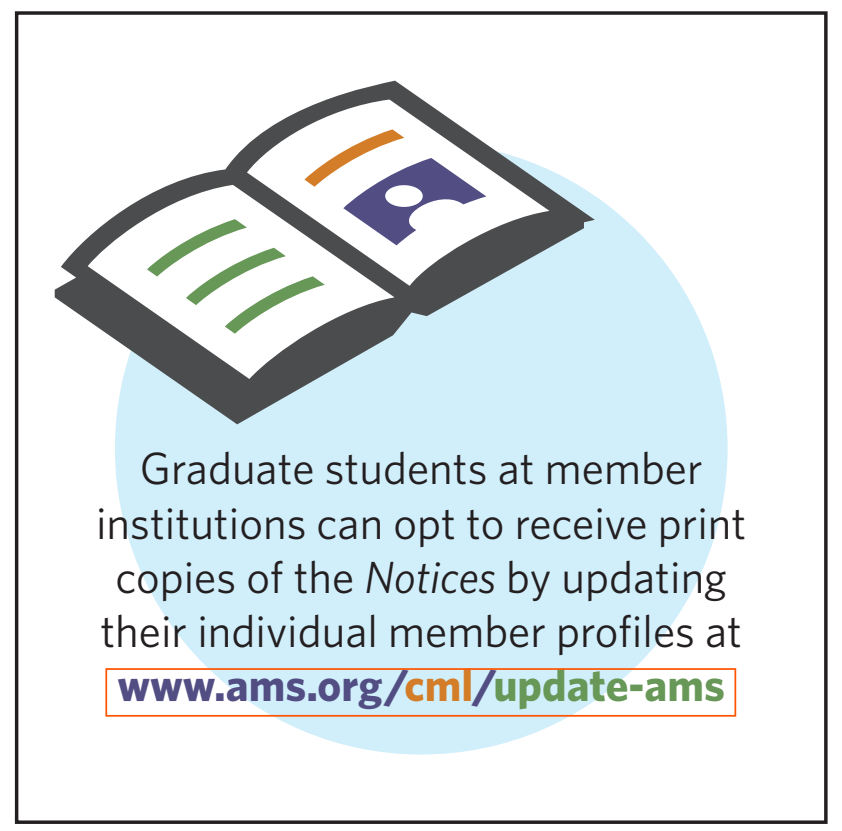




\section{THE GRADUATE STUDENT SECTION}

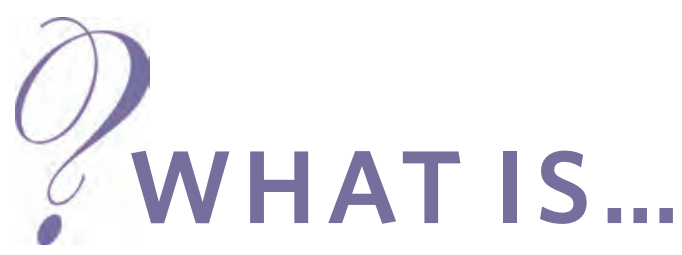

\section{a Rectifiable Set?}

Editors

A rectifiable set is our best definition of a generalized $k$-dimensional surface in $\mathbb{R}^{n}$, perhaps with infinitely many singularities and infinite topological type, but still nice enough to do differential geometry. While smooth surfaces can be defined locally as images of nice smooth functions from the unit ball in $\mathbb{R}^{k}$ into $\mathbb{R}^{n}$, rectifiable sets are defined as countable unions of images of Lipschitz functions from arbitrary subsets of $\mathbb{R}^{k}$ into $\mathbb{R}^{n}$. A Lipschitz function $f$ by definition satisfies

$$
|f(y)-f(x)| \leq C|y-x| .
$$

There is also a condition that a rectifiable set have finite $k$-dimensional measure (technically given by Hausdroff measure).

The simplest nonsmooth example of a Lipschitz function from $\mathbb{R}^{1}$ to $\mathbb{R}^{2}$ is $f(x)=(x,|x|)$ as in Figure 1 ; its image has a singularity at the origin. A 1-dimensional rectifiable set is a countable union of continuous curves of finite total length. A 2-dimensional rectifiable set can have dense singularities and infinite topological type, as in Figure 2.
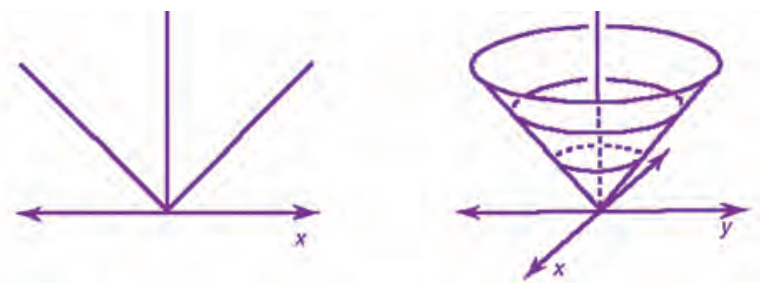

Figure 1. A rectifiable set, as the image of a Lipschitz function, can have sharp corner singularities.

Rademacher's Theorem says that a Lipschitz function is differentiable almost everywhere. The corresponding fact about rectifiable sets is the existence of an approximate tangent plane at almost every point $p$. Approximate means

For permission to reprint this article, please contact:

reprint-permission@ams .org.

DOI: http://dx.doi.org/10.1090/noti1730

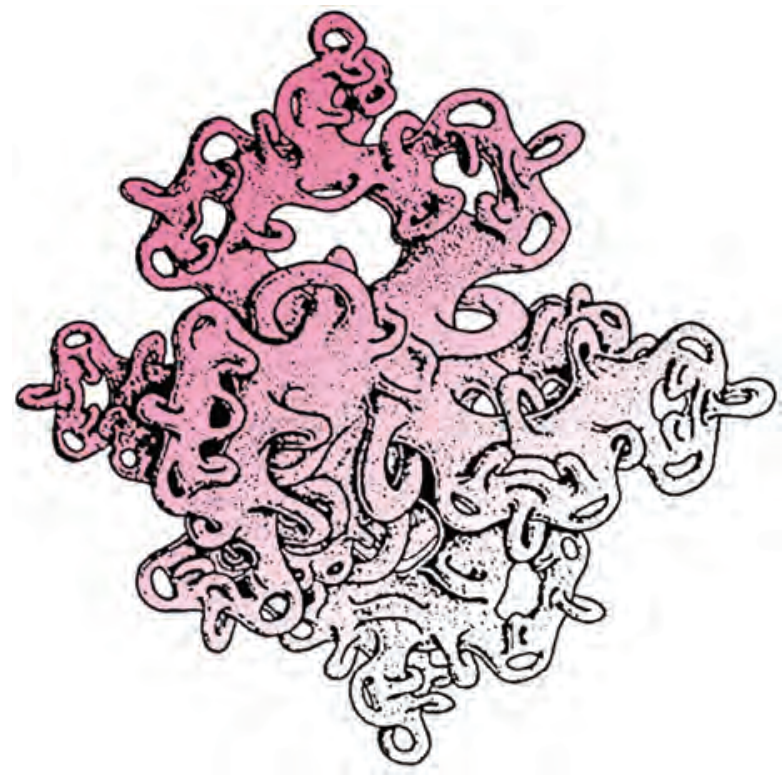

Figure 2. Adding infinitely many handles of finite total area to the sphere can produce a rectifiable set $S$ with infinite topological type and a singular dense Cantor set of positive measure. Nevertheless, $S$ has an approximate tangent plane at almost all points.

ignoring sets of density 0 at $p$. This fact means that rectifiable sets admit a measure theoretic generalization of differentiable geometry. It also means that you can integrate smooth differential forms over rectifiable sets and thus view them as the so-called rectifiable currents of geometric measure theory.

Besicovitch and Federer proved an amazing structure theorem, which says that every set of finite $k$-dimensional measure can be decomposed into a rectifiable set and a purely unrectifiable set. The purely unrectifiable set has the property that it is invisible from almost every direction, i.e., that its projection onto almost every $k$-plane 


\section{THE GRADUATE STUDENT SECTION}

has measure 0 . Cantor sets provide examples of purely unrectifiable sets, as in Figure 3.

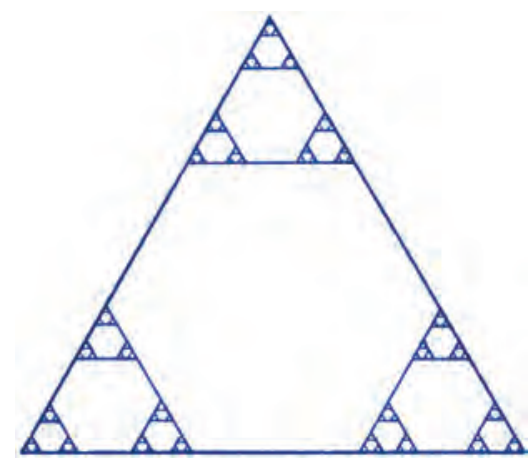

Figure 3. The pictured Cantor set, obtained by successively removing all but the three small corners of every triangle, is a purely unrectifiable set of unit 1-dimensional Hausdorff measure. Its projection onto almost every line has measure 0 ; a horizontal line is one exception.

Federer and Fleming provided an amazing compactness theorem for nice rectifiable sets with multiplicity in a ball in $\mathbb{R}^{n}$ in the context of geometric measure theory. By "nice" one means that in an appropriate sense the boundaries also are rectifiable. The only other hypothesis is a bound on the measures of the sets and of their boundaries. Then every sequence has a convergent subsequence in an appropriate weak topology, given by the so-called flat norm. For example, the flat distance between two close concentric spheres is the volume between them. In this norm, the exotic surface of Figure 4 and the flat disk are close together, because the region between them has small volume. This compactness theorem can be used to show that many problems have solutions. For example, every smooth closed curve in $\mathbb{R}^{3}$ is the boundary of a surface of least area.

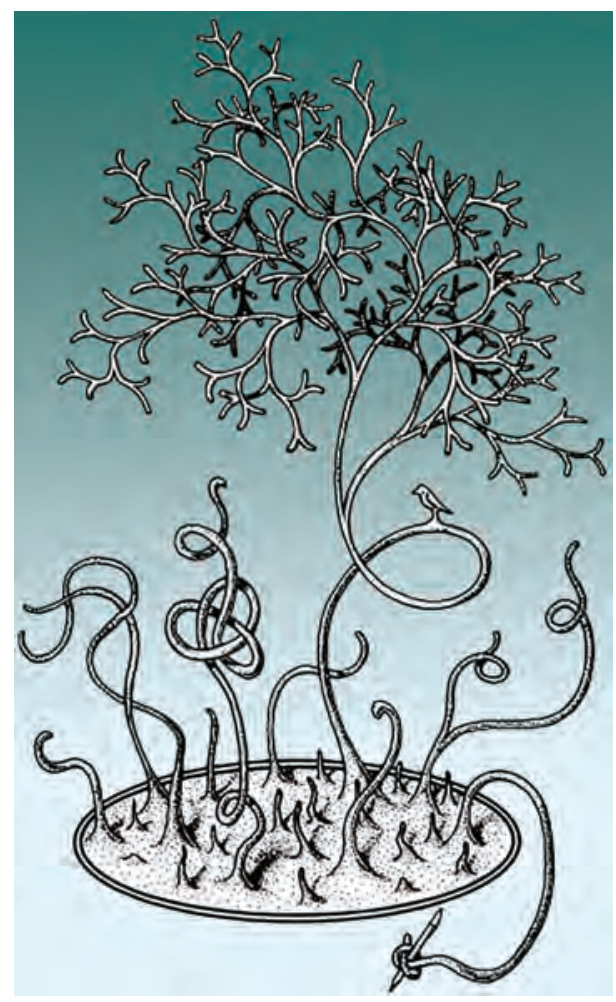

Figure 4.This exotic surface with its long thin tentacles is close to the planar disc in the flat norm, because the region between them has small volume.

All of these notions and results have generalizations from $\mathbb{R}^{n}$ to nice Riemannian manifolds. Although solutions to geometric problems need not always be smooth, much current regularity theory focuses on proving them rectifiable.

\section{References}

[1] Morgan, Frank, Geometric Measure Theory: A Beginner's Guide, 5th ed., Academic Press, 2016.

\section{Image Credits}

Figures by James F. Bredt, copyright Frank Morgan. All rights reserved. 


\title{
Geometry Labs United: An Invitation
}

\author{
Jayadev Athreya, David Dumas, William Goldman, \\ Sergey Grigorian, Rosemary Guzman, Philipp Hieronymi, \\ Sean Lawton, Anton Lukyanenko, Jeremy Tyson, and Aaron Wilson
}

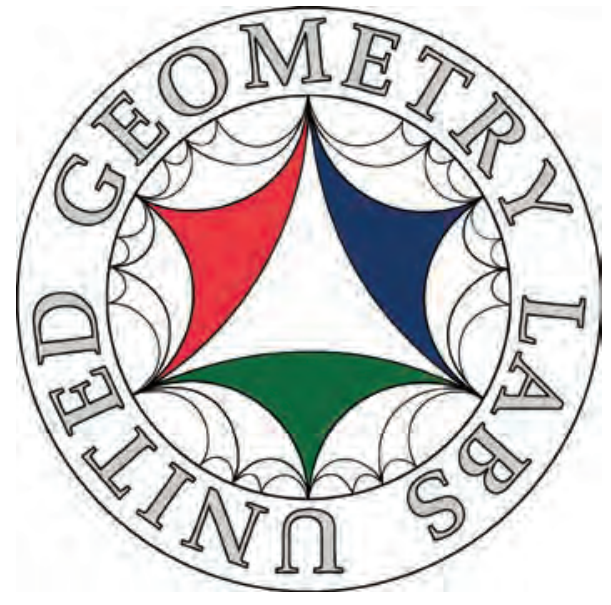

The purpose of a Geometry Lab is to foster a community to promote research involving all stages of academia, provide mentoring experiences to early career mathematicians, and disseminate mathematics beyond the boundaries of the university. Breakthroughs in science and mathematics often come from experimentation, computation, and communication of technical facts to non-experts. Geometry Labs (also known as Experimental Mathematics Labs) espouse this approach with vertically integrated groups of students and researchers advancing mathematics through experimentation, visualization, and public engagement.

Jayadev Athreya is associate professor at the University of Washington. His email address is jathreya@uw. edu.

David Dumas is professor in the Department of Mathematics, Statistics, and Computer Science at the University of Illinois at Chicago. His email address is david@dumas . io.

Philipp Hieronymi is associate professor at the University of Illinois at Urbana-Champaign. His email address is phierony@ i11inois. edu.

William Goldman is professor at the University of Maryland, College Park. His email address is wmg@math. umd. edu.

Sergey Grigorian is assistant professor at the School of Mathematical and Statistical Sciences, University of Texas Rio Grande Valley. His email address is sergey.grigorian@utrgv.edu.

For permission to reprint this article, please contact: reprint-permission@ams.org.

DOI: http://dx.doi.org/10.1090/noti1733
The research community at the core of an experimental mathematics lab produces a synergistic experience: the undergraduates' research resonates with their coursework; graduate students' research blends with mentoring; and faculty foster a deeper connection between research and teaching. In parallel, this shared experience informs public engagement activities that bridge the gap between science and society, paving the way for the next generation of researchers.

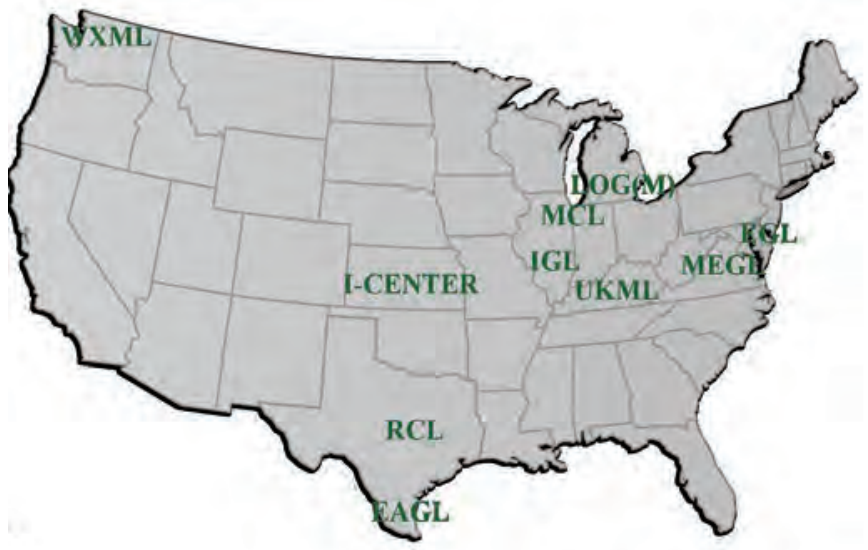

Figure 1: The ten member labs of the Geometry Labs United (GLU) network.

Rosemary Guzman is a postdoctoral fellow in the Department of Mathematics at the University of Chicago. Her email address is guzman@math. uchicago.edu.

Sean Lawton is associate professor at George Mason University. His email address is s1 awton3@gmu. edu.

Anton Lukyanenko is assistant professor at George Mason University. His email address is a 1ukyane@gmu.edu.

Jeremy Tyson is professor and mathematics department chair at the University of Illinois at Urbana-Champaign. His email address is tyson@i11inois.edu.

Aaron Wilson is assistant professor at the School of Mathematical and Statistical Sciences, University of Texas Rio Grande Valley. His email address is aaron.wi1son@utrgv. edu. 
Through this community and shared experience, geometry labs make research and geometry, broadly understood, more accessible. For all students, and especially for those under-represented in STEM, geometry labs serve as an important bridge toward higher mathematics. The emphasis on collaboration, active and experiential research, and outreach make geometry labs both a microcosm of the larger mathematical research community and an example of what it can be. To showcase this diversity, we highlight some of the students and projects, focusing on recent developments in GLU's 18-year history.

\section{Samuel J. Cavazos, Experimental Algebra and Geometry Lab}

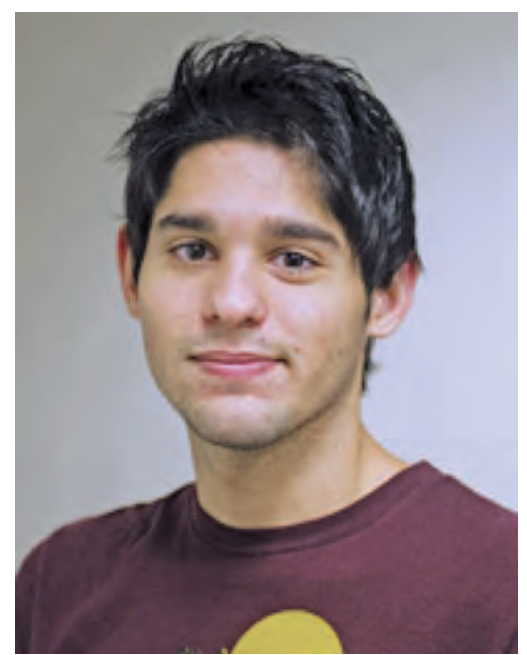

Figure 2: Samuel J. Cavazos found a formula for the number of representations of free groups over finite fields.

Sam Cavazos (Figure 2) was a member of the Experimental Algebra \& Geometry Lab (EAGL) at the University of Texas Rio Grande Valley (UTRGV) from the spring of 2011 to the summer of 2013. During Sam's first year in college he began working with Sean Lawton in EAGL. He was given the task of counting representations of free groups over finite fields (Figure 3). He quickly learned to write code in the computer program Mathematica and conjectured the correct formula by analyzing patterns generated by his program. In his second year, Sam began the project which led to his publication "E-polynomial of SL(2,C)-character varieties of free groups," Internat. J. Math. 25 (2014). This result gives an exact counting polynomial over all finite fields and for all free groups of the conjugation orbits of the aforementioned space of representations, as well as the point count of the associated quotient in the category of algebraic varieties. Such spaces are called character varieties, central objects in differential geometry and mathematical physics.

Based on his work in EAGL, Sam won a National Science Foundation Graduate Fellowship in 2013. He completed his undergraduate studies in just three years and graduated Summa Cum Laude with a BS in Pure Mathematics and a minor in Philosophy. Sam went on to Northwestern University, earned a Master's degree in mathematics in 2015, and is now a lead data analyst in Chicago.

Sam's research at UTRGV was supported four times by the Louis Stokes Alliances for Minority Participation (LSAMP) Program. He presented his work at the LSAMP conference, the Young Mathematicians Conference, the Society for Advancement of Chicanos and Native Amer- icans in Science (SAC-

NAS) conference,

the Hispanic,

Engineering,

Science, and

Technology

(HESTEC)

Week, and

numerous

faculty and

$\mathrm{s} t \mathrm{u}$ d e n t

$\mathrm{res}$ e a r c h

seminars at

UTRGV. He won

the top research

prizes for his poster

and presentations at

HESTEC and SACNAS

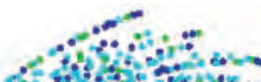

(receiving national attention). Additionally, Sam gave mathematical community outreach presentations to middle school students and teachers through EAGL's outreach endeavors.

According to Sam, "The lessons I learned during my time at the Experimental Algebra and Geometry Lab extend far beyond the world of mathematics. It taught me how to solve problems that make a positive impact on my community."

\section{Kristine Hampton, Washington Experimental Mathematics Lab (WXML)}

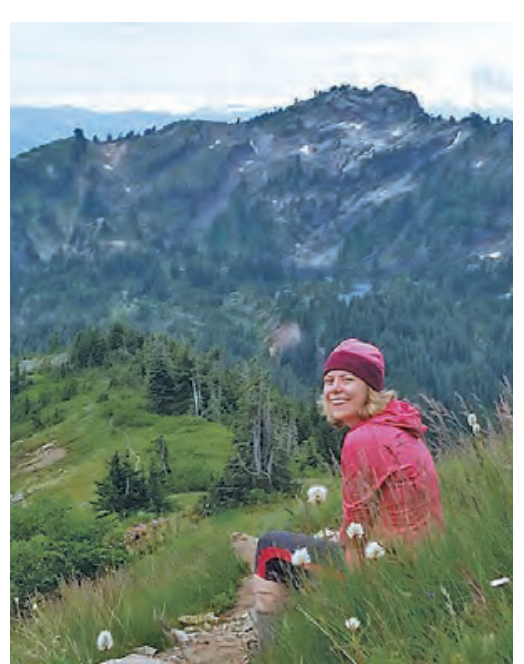

Figure 4: Kristine Hampton

studied the statistical

properties of the discrete

logarithm viewed as a

permutation.

Kristine Hampton (Figure 4) joined the WXML at the University of Washington in its first quarter of existence, Spring 2016. She worked alongside Matthew Ogi and Qiang $\mathrm{Yu}$ on a project co-mentored by Jayadev Athreya, Chris Hoffman, Jacob Richey, and Dan Bragg, studying the statistical properties of the discrete logarithm viewed as a permutation. This project combined number theory, group theory, combinatorics, probability, and programming. The project developed into a study of the actions of linear groups over finite fields on projective spaces, motivated by questions of V.I. Arnold. Kristine led the team through fascinating numerical and theoretical questions, particularly analyzing the number of cycles and number of fixed points in the discrete logarithm, and comparing them to those of 


\section{Average Length of Longest Cycle}

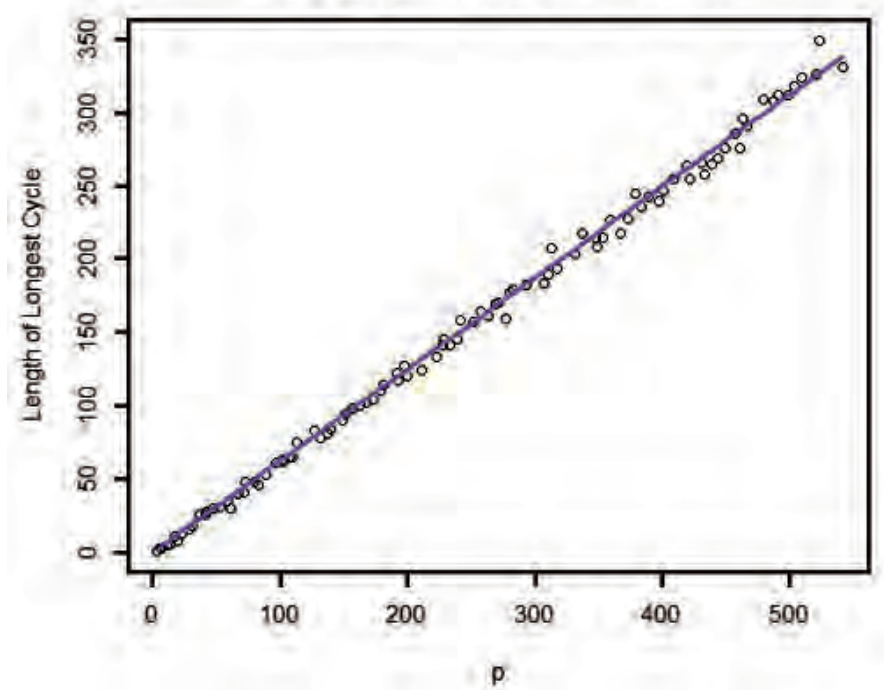

Average Number of Fixed Points

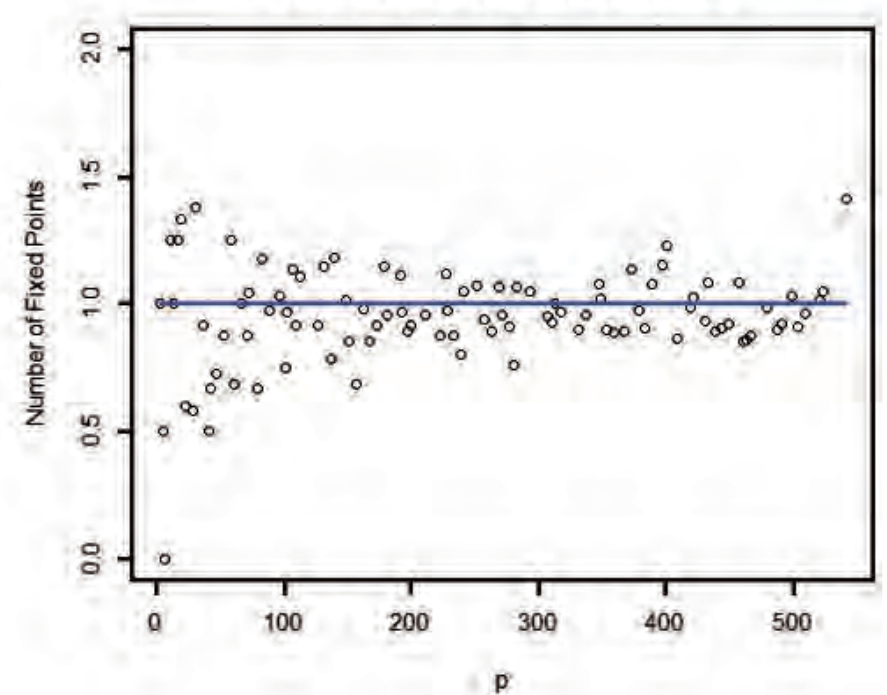

Figure 5: Asymptotics of number of cycles and length of longest cycles in discrete logarithms.

randomly chosen permutations (Figure 5 ). After working with the WXML through the 2016-17 academic year, Kristine joined UW as a PhD student in Autumn 2017, where she is planning on studying algebraic geometry.

In addition to her research work, Kristine has helped start and develop the WXML and "Math for Love" math teacher circles, a monthly math circle for elementary and middle school math teachers. These math circles, managed by Christine, are a collaboration between "Math for Love," a Seattle-based mathematics organization, and the WXML. Kristine was instrumental in securing funding from the American Institute of Mathematics and also getting the participating teachers professional development credits. She continues to successfully manage the math circles as a PhD student.

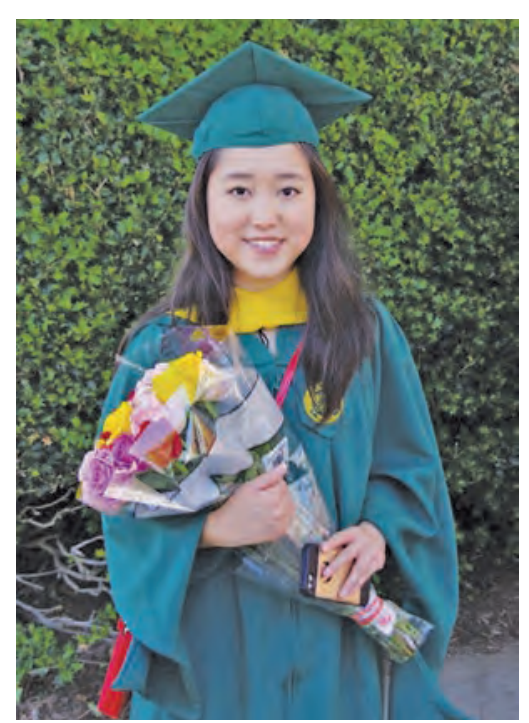

Figure 6: Stephanie Mui created sinusoidal embeddings of flat tori and Nash spheres in 3D.

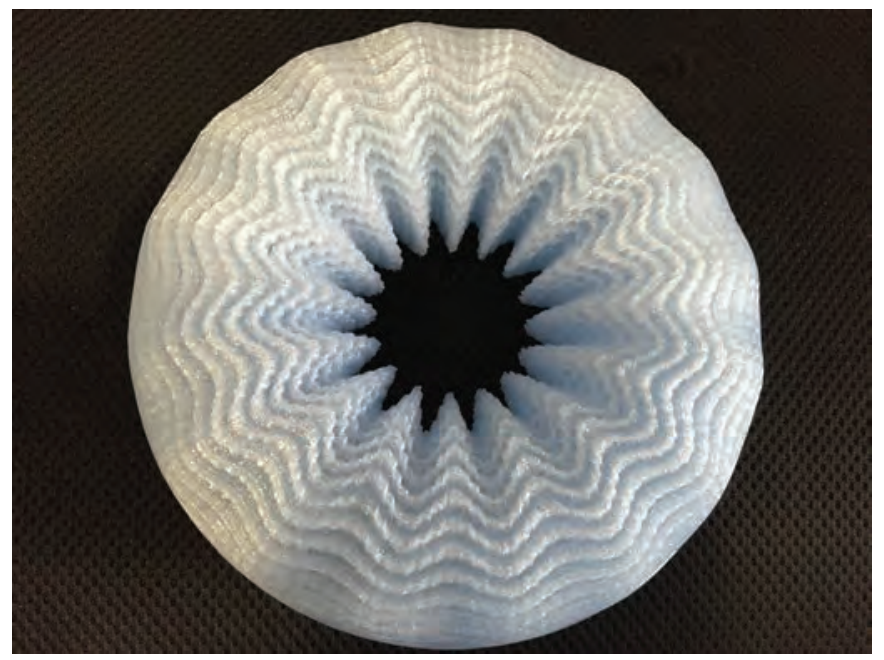

Figure 7: A 3D print of a flat torus.

award of US\$2,000 from the American Mathematical Society.

Her thesis research concerned visualizing embedded geometric manifolds. She used computer programming and 3D printing to experimentally explore her ideas as she studied relevant theory and developed new ideas. See meglab.wikidot.com/visualization for examples of her 3D prints and stereographic 3D movies.

When Stephanie was not working on research or earning straight A's, she assisted with MEGL's community outreach, inspiring young people in the community to appreciate mathematics. She also gave many research presentations including a talk at the Undergraduate Mathematics Symposium at the University of Illinois at Chicago.

Stephanie graduated Summa Cum Laude, earning both a BS in Pure Mathematics with honors and an MS in Mathematics. After graduating Stephanie went to work on her $\mathrm{PhD}$ at the Courant Institute of Mathematical Sciences at NYU with a full research fellowship. Stephanie was the 
youngest graduate in GMU's history at age 17; in fact, she earned her MS and BS before her high school diploma.

According to Stephanie, "MEGL is the prime reason I am continuing my studies in math today. My mentor at MEGL sparked my interest in differential geometry and provided me the rigorous training and insightful roadmap to conduct research."

\section{Matthew Staples,} Washington Experimental Mathematics Lab Matthew Staples (Figure 8) joined the WXML at the University of Washington in its first quarter of existence, Spring 2016, working alongside Wenbo Gao and Maria Osborne on a project mentored by Jayadev Athreya and Dia Taha. Matt's group worked to understand the properties of the new pentagonal tiling of the plane recently discovered by Mann, McLoud, and Von Derau at the University of Washington Bothell (also as part of an undergraduate research project). They studied

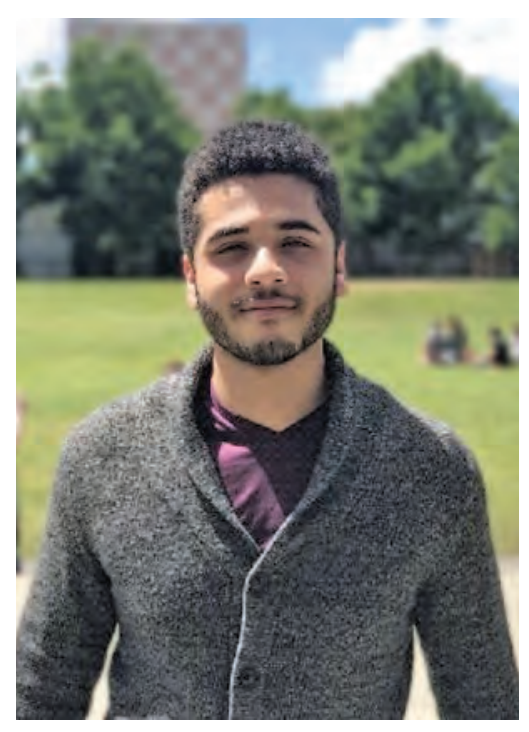

Figure 8: Matthew Staples studied properties of a new pentagonal tiling of the plane showing that the associated surface has a large group of symmetries. statistical properties of this tiling and the billiard flow in this rational pentagon, and showed that the associated surface had a large group of affine symmetries.

In addition to his WXML research work, Matt helped develop WXML's website and worked on several 3D printing and fabrication projects. Matt graduated from UW in summer of 2016, and is currently an associate data warehouse analyst at the Comtech Telecommunications Corporation in Seattle.

According to Matt, "Being a part of WXML was the best part of my university experience."
Racquetball in the

3-torus, Mathematical

Computing Laboratory

In this project at the Mathematical Computing Lab at the University of Illinois at Chicago, undergraduate researchers Horalia Armas and Brandon Reichman worked with graduate mentor Hai Tran and faculty supervisor David Dumas (Figure 10) on a project to demonstrate features of multiply-connected spaces and curved geometries using virtual reality.

Despite starting the project with no game development or 3D graphics experience, Armas and Reichman embraced the challenge and had considerable success. Using the Unity 3D engine and its associated integrated development environment, they created a first-person VR racquetball game that can be played in the 3-dimensional torus and several other Euclidean orbifold geometries.

Notably, this racquetball game is designed for a single player; the ball is passed back and forth along closed geodesics in the ambient space. By taking advantage of the

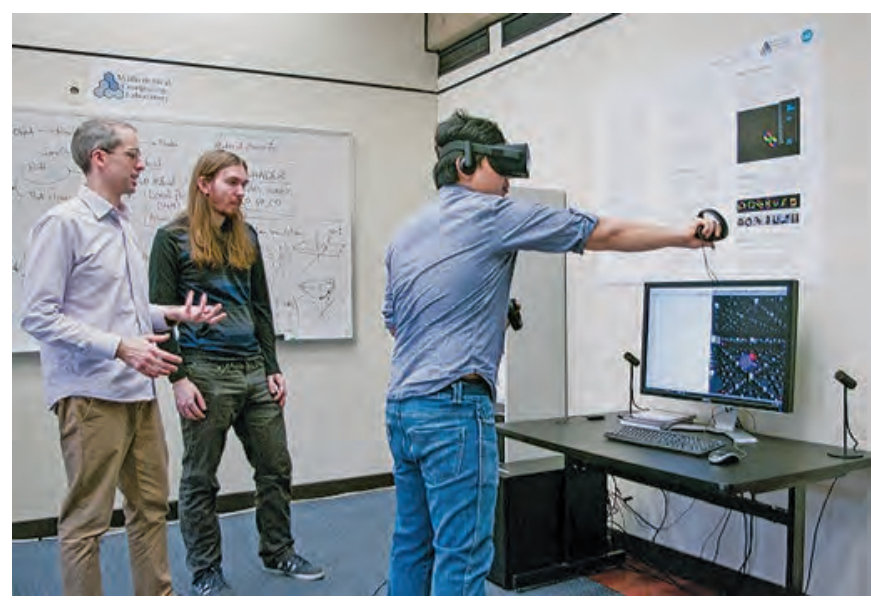

Figure 10: Faculty supervisor David Dumas, undergraduate Brandon Reichman, and graduate mentor Hai Tran, along with undergraduate Horalia Armas (not pictured here) developed a virtual reality ManifoldBall to understand multiply connected spaces and curved geometries. 


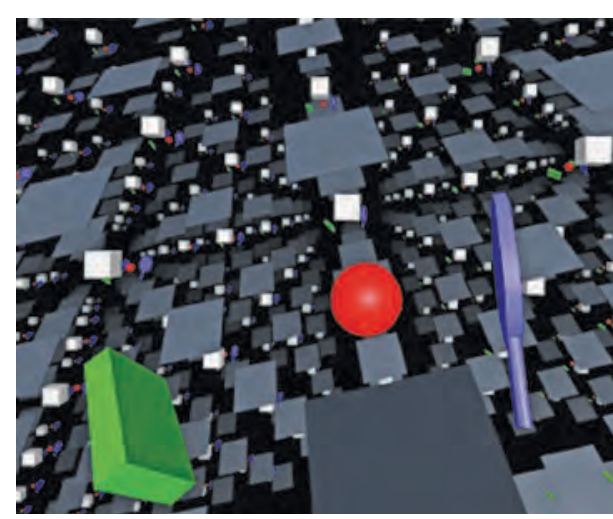

Figure 11: In-game screenshot of ManifoldBall.

player's expectations about the basic goal of the game (i.e. to hit the ball), the game embeds exploration of the geometry of the 3-torus in a familiar and recreational setting.

The resulting game, ManifoldBall, and the group's project were featured in a March 2017 article in the journal Nature. ${ }^{1}$

To play the game, visit mc1.math.uic.edu/spring -2017-projects.

\section{Statistical Quantum Mechanics for Graphs and CW-Complexes, Illinois Geometry Lab}

In this project at the Illinois Geometry Lab (IGL) at the University of Illinois at Urbana-Champaign, the research team led by faculty mentor Ivan Contreras and graduate team leader Sarah Loeb (now a postdoc at the College of William \& Mary) developed a model for quantum mechanics and

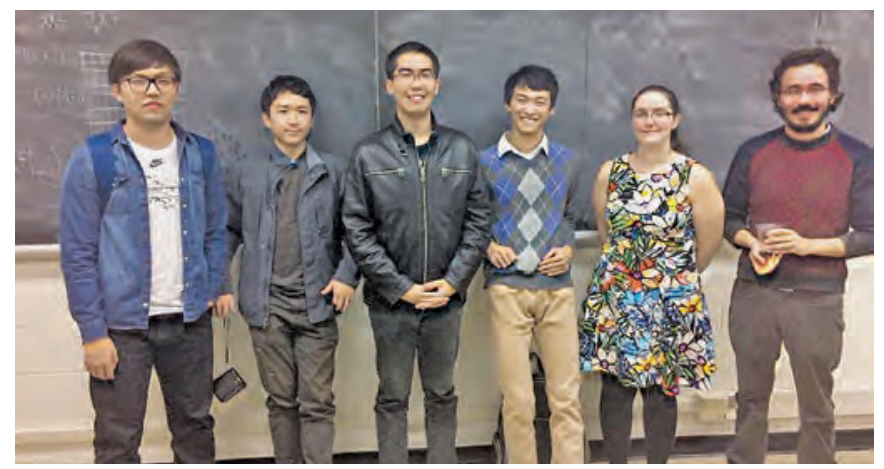

Figure 12: The research team Statistical Quantum Mechanics for Graphs and CW-Complexes developed a machine-learning based algorithm to apply graph quantum mechanics to text analysis.

thermodynamics. In this model, the physical system is confined to a finite graph or a CW-complex, and the evolution of the system is prescribed by a discretized version of the Schrödinger or heat equation, respectively. The team used this model to better understand the topology of graphs and $\mathrm{CW}$-complexes, as well as to provide a combinatorial

\footnotetext{
${ }^{1}$ Mathematicians create warped worlds in virtual reality, Nature 543, 473 (23 March 2017). https://www. nature.com/news /mathematicians-create-warped-worlds-in-virtual -reality-1.21689.
}

meaning to the partition function of quantum mechanics and thermodynamics (Figure 13). As an application, they developed a machine-learning based algorithm to apply graph quantum mechanics to text analysis.

Three papers resulted from this research, all including undergraduates as co-authors. Undergraduate students in the team have presented their work at various regional and national conferences. In particular, Rodrigo Araiza presented at the Latinx Conference, and Chengzheng $\mathrm{Yu}$ at the 2018 Joint Math Meetings. In addition, undergraduate Michael Toriyama won a prestigious Barry Goldwater Scholarship, and his fellow team member Boyan $\mathrm{Xu}$ is starting graduate studies in mathematics at UC Berkeley in Fall 2018. This project exemplifies the IGL's mission to make substantial contributions to mathematical knowledge, while simultaneously fostering the professional development of both undergraduate and graduate students.

\section{How Does a Lab Take Shape?}

The narratives above demonstrate the vast diversity of Experimental Mathematics Labs: their projects, participants, and experiences. Likewise, the labs themselves take many shapes, and each one develops based on the resources, needs, and interests of its department. The goal of a founding director is to leverage these components to build a vertically-integrated research community focused on visualization, computation, and public engagement. The particular way in which this happens varies from lab to lab.

Ultimately, an Experimental Mathematics Lab is an organization that facilitates vertically integrated projects within a mathematics department. An approach used by most labs is to run term-long projects during the academic year. These projects are led by a faculty member, overseen if possible by a graduate student, and implemented by a team of 2-4 undergraduate students earning course credit or financial compensation.

Ideally, everyone is engaged at the level and time commitment appropriate to their experience: the undergraduate students work up to 10 hours per week and contribute computations and technology expertise; the graduate student guides the team, exploring and explaining relevant theory and meeting with the undergraduates for one hour a week; the faculty mentor oversees the experience, 
suggesting the initial problem and regularly joining the weekly meetings.

The lab is run by a faculty director, with further assistance from faculty members, graduate students, or undergraduate students according to the size of the lab. To prepare for each new term, lab leadership solicits projects from faculty, advertises to the students, and forms collaborative teams. During the semester, consultation is provided to teams, and all-lab meetings are arranged to showcase progress to the lab's members, culminating in a broadly-advertised public poster session or a research symposium.

In parallel, the lab leadership (often involving a dedicated outreach manager) develops and coordinates community partnerships and engagement activities, providing a bridge between university mathematics and the broader public. These activities are implemented by the outreach manager, as well as volunteer lab members at all levels. The community engagement has two important effects: the university students leading the activities gain confidence in their own ability to do and present mathematics, and the K-12 activity participants show improved attitudes towards mathematics through the near-peer effect. ${ }^{2}$

To build up to this level of infrastructure, a founding director must ignite interest within their department. Initial investment by the department and student body can be created by showcasing projects from other labs and advertising the use of cutting-edge technology in research (such as 3D printing, virtual reality, and parallel computing). Once this advertising creates a seed community of interested parties, projects can be started, and, if possible, a space can be established for joint meetings, presentations, and collaborative student work. While such a space can be hard to obtain, and strictly speaking is not necessary, it is useful in creating a cohesive, vertically integrated community that facilitates sharing of expertise across projects.

\section{Call for More Labs}

If you're interested in developing your own experimental mathematics lab, GLU can help. We encourage you to join us at our special session at the 2019 Joint Mathematics Meetings in Baltimore (January, 2019) or the bi-annual GLU Conference (Summer 2019) at George Mason University; visit us at geometrylabs. net (where you can find a lab starter kit, created by Dia Taha, Leif Svensson, Jonah Ostroff, and the WXML leadership team, building on materials created at the IGL by Noel DeJarnette, Anton Lukyanenko, and Grace Work), or email us at glu@geometrylabs . net.

\footnotetext{
${ }^{2}$ A. Wilson \& S. Grigorian, The near-peer mathematical mentoring cycle: studying the impact of outreach on high school student attitudes toward mathematics, Int. J. Math. Ed. Sci. Tech. 49 (2018).
}

\section{ABOUT THE AUTHORS}

Jayadev Athreya co-founded the Illinois Geometry Lab, and directed it from 2011 to 2015. In 2012, he co-founded Geometry Labs United. He founded the Washington Experimental Mathematics Lab in 2015, and has directed it since. He sees experimental mathematics labs as vehicles for advancing equity and diversity in mathematics.

David Dumas is a co-founder of the Mathematical Computing Laboratory at University of Illinois at Chicago.

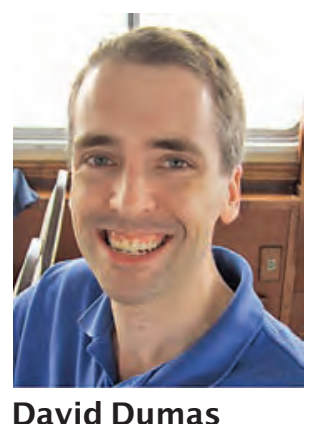

Philipp Hieronymi is the director of the Illinois Geometry Lab.

William Goldman directs the Experimental Geometry Lab. He studied with Bill Thurston, Dennis Sullivan, and Morris Hirsch at Princeton and Berkeley in the 1970s. In 2000 he co-founded the EGL, where he mentored Sean Lawton and Anton Lukyanenko. In 2012, after three geometry labs had been formed, he co-founded Geometry Labs United. He co-directed a summer 2018 REU at ICERM at Brown University.

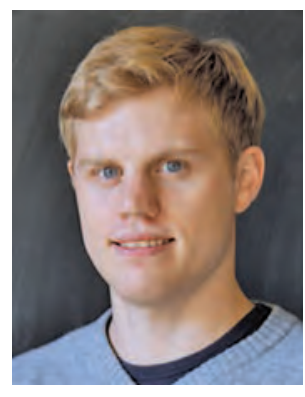

Philipp Hieronymi

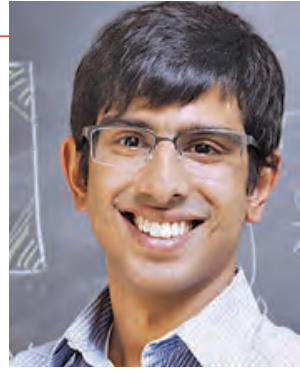

Jayadev Athreya

David Dumas

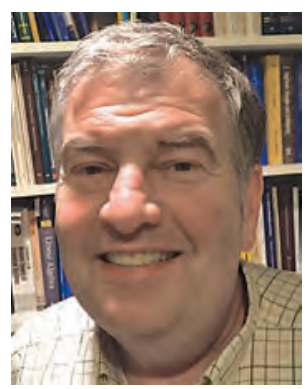

William Goldman 


\section{COMMUNICATION}

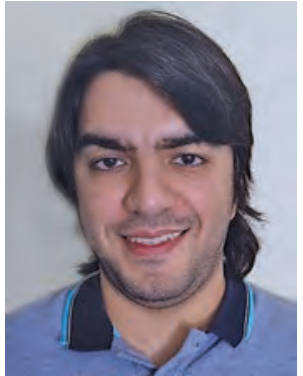

Sergey Grigorian

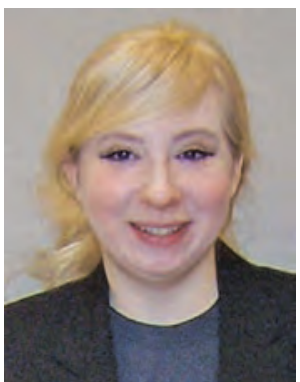

Rosemary

Guzman

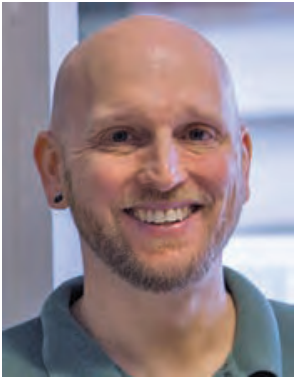

Sean Lawton was the founding director of the Experimental Algebra \& Geometry Lab in Texas from 2009 to 2014. In 2012, he co-founded Geometry Labs United. In 2014 he founded the Mason Experimental Geometry Lab and has since directed it. He studied with William Goldman at the Experimental Geometry Lab in Maryland as a student and post-doc.

Sean Lawton
Sergey Grigorian is currently a co-director of the Experimental Algebra and Geometry Lab. He is working to integrate geometry lab activities with the university curriculum within a community engaged scholarship framework.

Rosemary Guzman served as assistant director of the Illinois Geometry Lab from 2015 to 2017.
Anton Lukyanenko is a past member of the Experimental Geometry Lab (as an undergraduate student), a co-founder of the Illinois Geometry Lab (as a graduate student) and the Laboratory of Geometry at Michigan (as a postdoc), and an assistant director of the Mason Experimental Geometry Lab (as a faculty member). In 2012, he co-founded Geometry Labs United.

Jeremy Tyson served as director of the Illinois Geometry Lab from 2015 to 2017.

Aaron Wilson is one of the current directors of the Experimental Algebra and Geometry Lab. He is interested in measuring the positive changes observed in students involved with the lab.

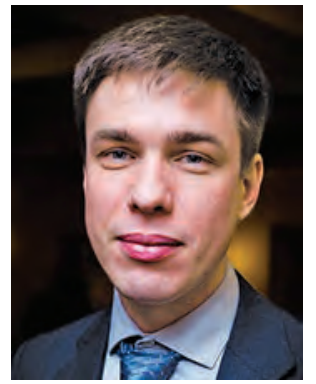

Anton

Lukyanenko

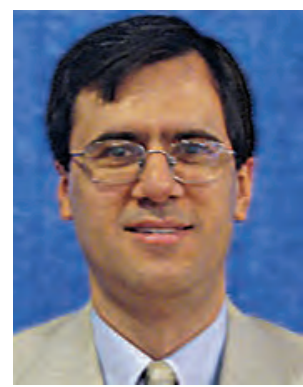

Jeremy Tyson

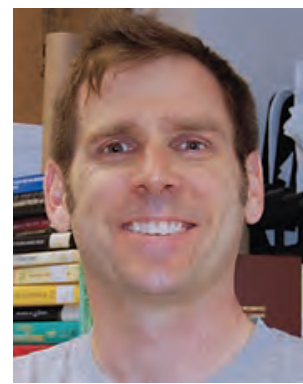

Aaron Wilson

\section{Image Credits}

GLU logo by Grace Work.

Figure 1 by Anton Lukyanenko and Sean Lawton.

Figure 2 courtesy of Sam Cavazos.

Figure 3 by Sam Cavazos.

Figure 4 by Nathan Redon. Figure 5 by Kristine Hampton, Matthew Ogi, Jacob Richey, and Qiang Yu at the WXML.

Figure 6 courtesy of Stephanie Mui.

Figure 7 photo by Sean Lawton.

Figure 8 courtesy of Matthew Staples.

Figure 9 by Wenbo Gao, Maria Osborne, Matt Staples, and Dia Taha, and the WXML.

Figure 10 courtesy of Hai Tran.

Figure 11 by Horalia Armas, David Dumas, Brandon Reichman, and Hai Tran.

Figures 12 and 13 by Ivan Contreras.

Photo of Jayadev Athreya courtesy of Jayadev Athreya. Photo of David Dumas courtesy of David Dumas. Photo of William Goldman by Evan Goldman. Photo of Sergey Grigorian courtesy of Sergey Grigorian. Photo of Rosemary Guzman by Tori Corkery. Photo of Philipp Hieronymi courtesy of Philipp Hieronymi. Photo of Sean Lawton by Duane King.

Photo of Anton Lukyanenko by Reider White.

Photo of Jeremy Tyson by Deb Kimme.

Photo of Aaron Wilson by Lezly Gonzalez. 


\section{The AMS Committee on Education invites you to attend the}

\section{ANNUAL MINI-CONFERENCE ON EDUCATION}

\section{"Next steps in the evolution of mathematics education: moving beyond pilots"}

In recent years, many innovations in mathematics education have been tried in different contexts. These innovations can serve as pilots for larger-scale changes in the teaching and learning of mathematics. This mini-conference will bring together some of the pioneers from mathematics and nearby fields to share their insights and experiences. The goal is to foster an open discussion and appraisal of which ideas should be scaled up in order for education in mathematics to meet future needs.

Join department chairs, directors of undergraduate and graduate studies, and others interested in education to hear from national leaders and to participate in lively discussions. We encourage small departmental teams to attend together.

\section{Registration Fee: $\$ 200$}

If you are interested in attending, please register by September 26, 2018, at http://bit.Iy/2JjKCJC.

\section{Friday, October 12, 2018 8:00 am-6:00 pm Washington, DC}

Confirmed speakers for the mini-conference include:

Christopher Edley, President, Opportunity Institute and former Dean of University of California, Berkeley School of Law

Ellen Hildreth, Professor of Computer Science, Wellesley College

Brit Kirwan, Chancellor Emeritus, University System of Maryland and Executive Director, TPSE Math

Sonin Kwon, Managing Director, Mass Mutual Financial Group

Rachel Levy, Deputy Executive Director, Mathematical Association of America

Jake Steel, Chief of Staff, Office of Planning, Evaluation and Policy Development, US Department of Education

Manil Suri, Professor of Mathematics, University of Maryland, Baltimore County

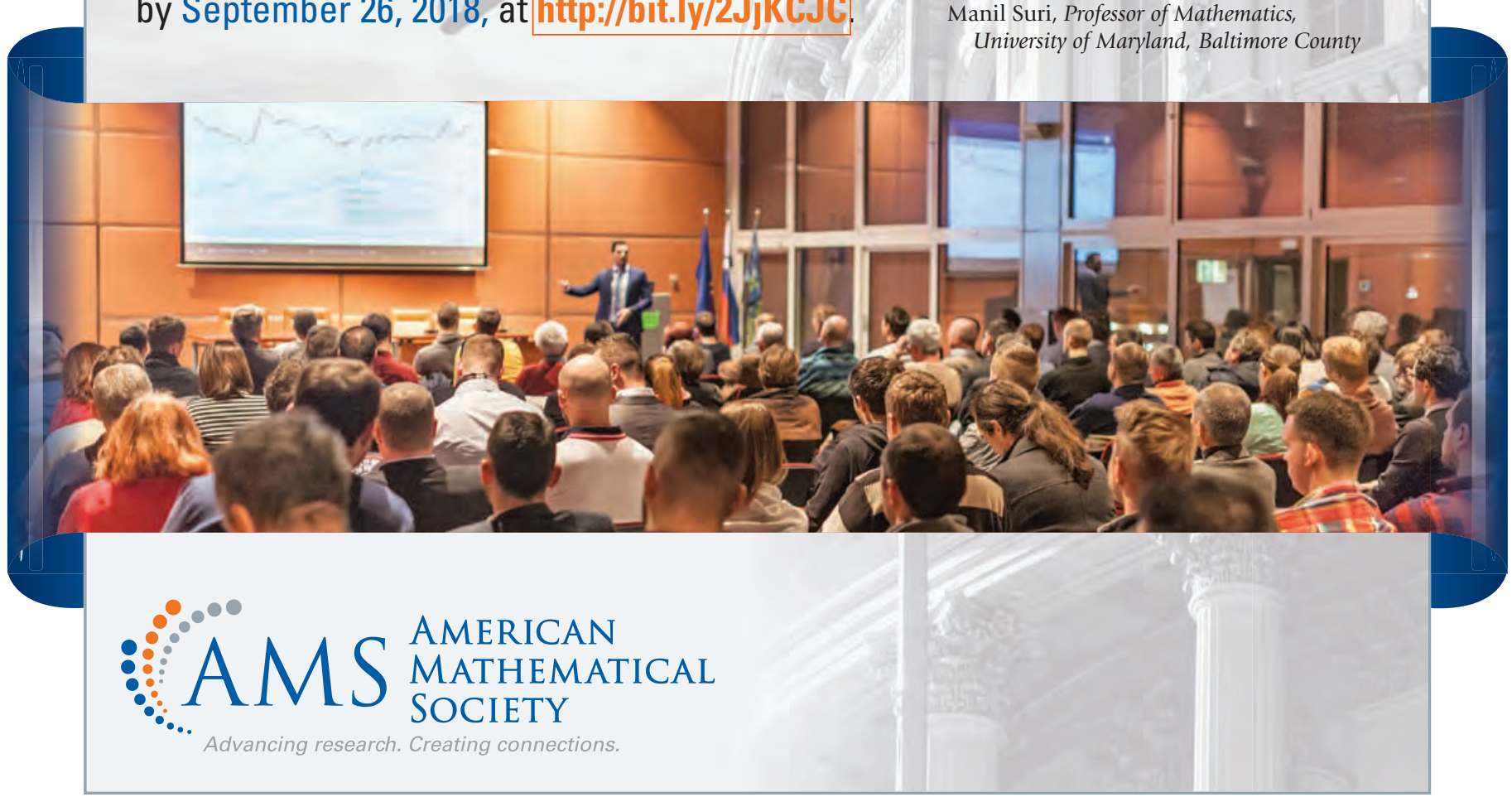




\section{Statistics on Women} Mathematicians

\section{Compiled by the AMS}

At its August 1985 meeting the Council of the AMS approved a motion to regularly assemble and report in the Notices information on the relative numbers of men versus women in at least the following categories: membership in the AMS, invited hour addresses at AMS meetings, speakers at Special Sessions at AMS meetings, percentage of women speakers in AMS Special Sessions by gender of organizers, and members of editorial boards of AMS journals.

It was subsequently decided that this information would be gathered by determining the gender of the individuals in the above categories based on name identification if no other means was available and that additional information on the number of PhDs granted to women would also be collected using the AMS-ASAIMS-MAA-SIAM Annual Survey. Since name identification was used, the information for some categories necessitated the use of three classifications:

Male: names that were obviously male

Female: names that were obviously female

Unknown: names that could not be identified as clearly male or female (e.g., only initials given, non-genderspecific names, etc.)

The following is the thirty-second reporting of this information. Updated reports will appear annually in the Notices.

\section{Trustees and Council Members}

\begin{tabular}{|c|c|c|c|c|c|c|c|}
\hline \multirow{3}{*}{ Male: } & \multicolumn{2}{|c|}{2017} & \multicolumn{2}{|c|}{2016} & \multicolumn{2}{|c|}{2015} & 2014 \\
\hline & 26 & $62 \%$ & 26 & $62 \%$ & 23 & $55 \%$ & 23 \\
\hline & $\begin{array}{l}16 \\
42\end{array}$ & $38 \%$ & $\begin{array}{l}16 \\
42\end{array}$ & $38 \%$ & & $45 \%$ & 18 \\
\hline
\end{tabular}

\begin{tabular}{lcr}
\hline & Invited Hour Address Speakers \\
& at AMS Meetings (2008-2017) \\
& 319 & $79 \%$ \\
Male: & 86 & $21 \%$ \\
Female: & 0 & $0 \%$ \\
Unknown: & 405 & \\
Total: & &
\end{tabular}

Speakers at Special Sessions

at AMS Meetings (2013-2017)

$\begin{array}{lrr}\text { Male: } & 12,924 & 72 \% \\ \text { Female: } & 3,929 & 22 \% \\ \text { Unknown: } & 1,206 & 7 \% \\ \text { Total: } & 18,059 & \end{array}$

Percentage of Women Speakers in AMS Special Sessions

by Gender of Organizers (2017)

Special Sessions

with at Least One Woman Organizer

$\begin{array}{lrr}\text { Male: } & 1,150 & 61 \% \\ \text { Female: } & 509 & 27 \% \\ \text { Unknown: } & 228 & 12 \% \\ \text { Total: } & 1,887 & \end{array}$

Special Sessions

with No Women Organizers

$\begin{array}{lrr}\text { Male: } & 1,471 & 67 \% \\ \text { Female: } & 393 & 18 \% \\ \text { Unknown: } & 318 & 15 \% \\ \text { Total: } & 2,182 & \end{array}$

Members of AMS Editorial Committees

\begin{tabular}{|c|c|c|c|c|c|c|c|c|c|c|}
\hline \multicolumn{11}{|c|}{ Members of AMS Editorial Committees } \\
\hline & 2017 & 2016 & 2015 & 2014 & 2013 & 2012 & 2011 & 2010 & 2009 & 2008 \\
\hline Male: & $17679 \%$ & $16180 \%$ & $17380 \%$ & $17981 \%$ & $18282 \%$ & $17883 \%$ & $17683 \%$ & $17682 \%$ & $17884 \%$ & $16883 \%$ \\
\hline Female: & $42 \quad 21 \%$ & $4120 \%$ & $4320 \%$ & $43 \quad 19 \%$ & $40 \quad 18 \%$ & $37 \quad 17 \%$ & $37 \quad 17 \%$ & $3918 \%$ & $34 \quad 16 \%$ & $35 \quad 17 \%$ \\
\hline Total: & 222 & 202 & 216 & 222 & 222 & 215 & 213 & 215 & 212 & 203 \\
\hline
\end{tabular}

2017 Members of the AMS Residing in the US

$\begin{array}{lrr}\text { Male: } & 8,591 & 40 \% \\ \text { Female: } & 1,694 & 7 \% \\ \text { Unknown: } & 13,674 & 57 \% \\ \text { Total: } & 23,959 & \end{array}$

PhDs Granted to US Citizens

\begin{tabular}{|c|c|c|c|c|c|c|c|c|c|c|c|c|c|c|c|}
\hline & 2017 & 2016 & 2015 & 2014 & 20 & & 20 & & 20 & & 20 & & 20 & & 2008 \\
\hline Male: & $64472 \%$ & $68473 \%$ & $63672 \%$ & $66472 \%$ & 627 & $73 \%$ & 621 & $72 \%$ & 574 & $72 \%$ & 566 & $71 \%$ & 515 & $69 \%$ & $43169 \%$ \\
\hline Female: & $24828 \%$ & $25127 \%$ & 244 & $28 \%$ & 230 & $27 \%$ & 242 & $28 \%$ & 228 & $28 \%$ & 226 & $29 \%$ & 227 & $31 \%$ & $19131 \%$ \\
\hline Other: & 1 & $2<1 \%$ & & & & & & & & & & & & & \\
\hline Total: & 893 & 937 & 880 & 920 & 857 & & 863 & & 802 & & 792 & & 742 & & 622 \\
\hline
\end{tabular}




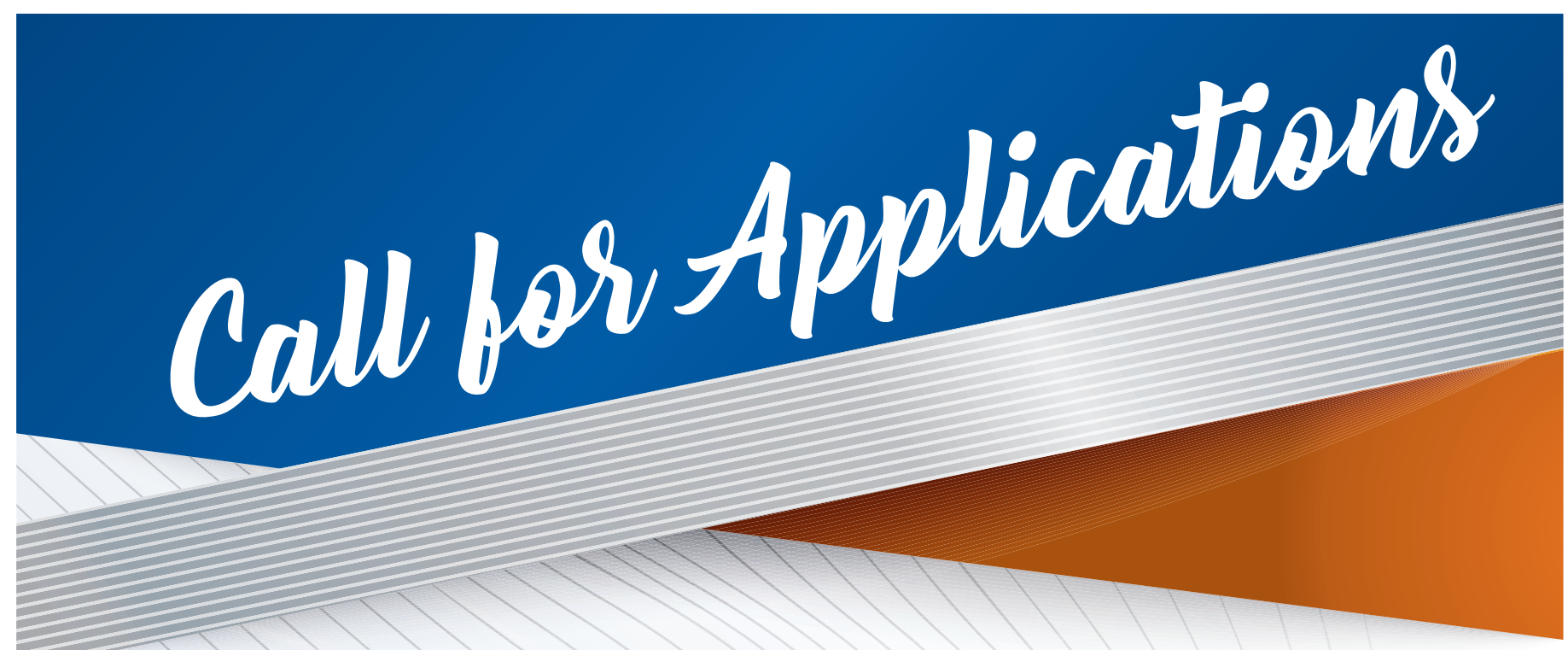

\section{THE 2019-20 JOAN AND JOSEPH BIRMAN FELLOWSHIP FOR WOMEN SCHOLARS}

The new Joan and Joseph Birman Fellowship for Women Scholars is a mid-career research fellowship specially designed to fit the unique needs of women. The fellowships are open only to women. This fellowship program, established in 2017, is made possible by a generous gift from Joan and Joseph Birman.

The fellowship seeks to address the paucity of women at the highest levels of research in mathematics by giving exceptionally talented women extra research support during their mid-career years.

The most likely awardee is a mid-career woman, based at a US academic institution, with a well-established research record in a core area of mathematics. The fellowship will be directed toward those for whom the award will make a real difference in the development of their research career. Candidates must have a carefully thought-through research plan for the fellowship period. Special circumstances (such as time taken off for care of children or other family members) may be taken into consideration in making the award.

The fellowship can be used to provide additional time for research of the awardee or opportunities to work with collaborators. This may include, but is not limited to, course buyouts, travel money, childcare support, or support to attend special research programs.

Further information and instructions for submitting an application can be found at the fellowship website: www.ams.org/Birman-fellow.

For questions contact the AMS Senior Program Coordinator Kim Kuda at prof-serv@ams.org. Application period: September 1-December 1, 2018

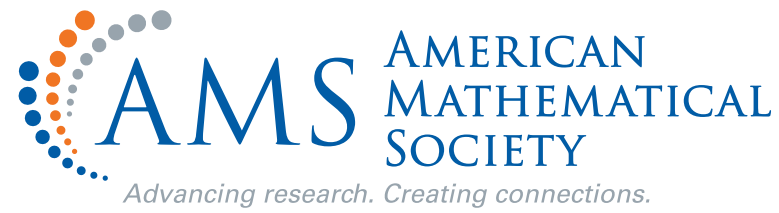




\section{Reverse Mathematics}

\section{Carl Mummert}

Communicated by Daniel J. Velleman

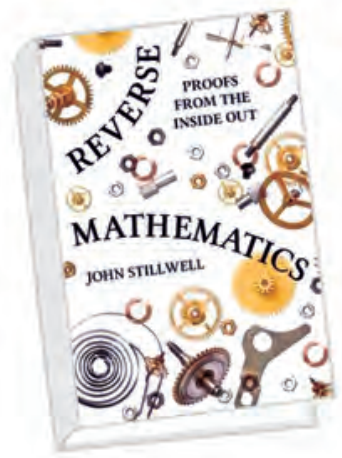

Reverse Mathematics: Proofs from the Inside Out

By John Stillwell

Princeton University Press

Hardcover, 200 pages

ISBN: 978-1-4008-8903-7

There are several monographs on aspects of reverse mathematics, but none can be described as a "general audience" text. Simpson's Subsystems of Second Order Arithmetic [3], rightly regarded as a classic, makes substantial assumptions about the reader's background in mathematical logic. Hirschfeldt's Slicing the Truth [2] is more accessible but also makes assumptions beyond an upperlevel undergraduate background and focuses more specifically on combinatorics. The field has been due for a general treatment accessible to undergraduates and to mathematicians in other areas looking for an easily comprehensible introduction to the field.

With Reverse Mathematics: Proofs from the Inside Out [5], John Stillwell provides exactly that kind of introduction. The book is aimed at upper-level undergraduates and professional mathematicians who are interested in the details of arithmetization and in seeing several examples of the methods used in reverse mathematics but who do not have previous knowledge of mathematical logic or computability theory.

\section{When Is an Axiom Necessary?}

Sometimes, when we prove a mathematical theorem, every step in the proof seems to be somehow required by the theorem at hand. Other times, we look at a particular step with skepticism. Have we used a sledgehammer to drive a nail, applying a very strong theorem to a problem that could be solved with simpler means? Are the techniques used in the proof genuinely necessary to obtain

Carl Mummert is associate professor of mathematics at Marshall University. His email address is mummert@marsha11. edu.

For permission to reprint this article, please contact:

reprint-permission@ams.org.

DOI: http://dx.doi.org/10.1090/noti1732 the theorem at hand? This question is a central motivation of the field of reverse mathematics in mathematical logic.

Mathematicians have long investigated the problem of the Parallel Postulate in geometry: which theorems require it, and which can be proved without it? Analogous questions arose about the Axiom of Choice: which theorems genuinely require the Axiom of Choice for their proofs?

In each of these cases, it is easy to see the importance of the background theory. After all, what use is it to prove a theorem "without the Axiom of Choice" if the proof uses some other axiom that already implies the Axiom of Choice? To address the question of necessity, we must begin by specifying a precise set of background axiomsour base theory. This allows us to answer the question of whether an additional axiom is necessary for a particular proof, as follows.

When a
theorem is
proved from
the right
axioms, the
axioms can be
proved from
the theorem.

Suppose we find that a theorem $T$ is provable from our base theory together with an additional axiom $A$ that is not provable in the base theory. To show that $A$ is necessary, we can try to prove a reversal: we assume $T$ as if it were an axiom, together with our base theory, and prove $A$ as if it were a theorem. If we can do this, we have shown that $A$ and $T$ are equivalent, relative to the base theory. Moreover, any other axiom $A^{\prime}$ which allows us to prove $T$ over our base theory would also allow us to prove $A$. The reversal shows that $A$ is, in a precise sense, the weakest axiom that, together with the base theory, allows us to prove $T$.

Many results of this kind have been obtained. For example, the Parallel Postulate is equivalent to Playfair's axiom in absolute geometry, and in topology the Axiom 


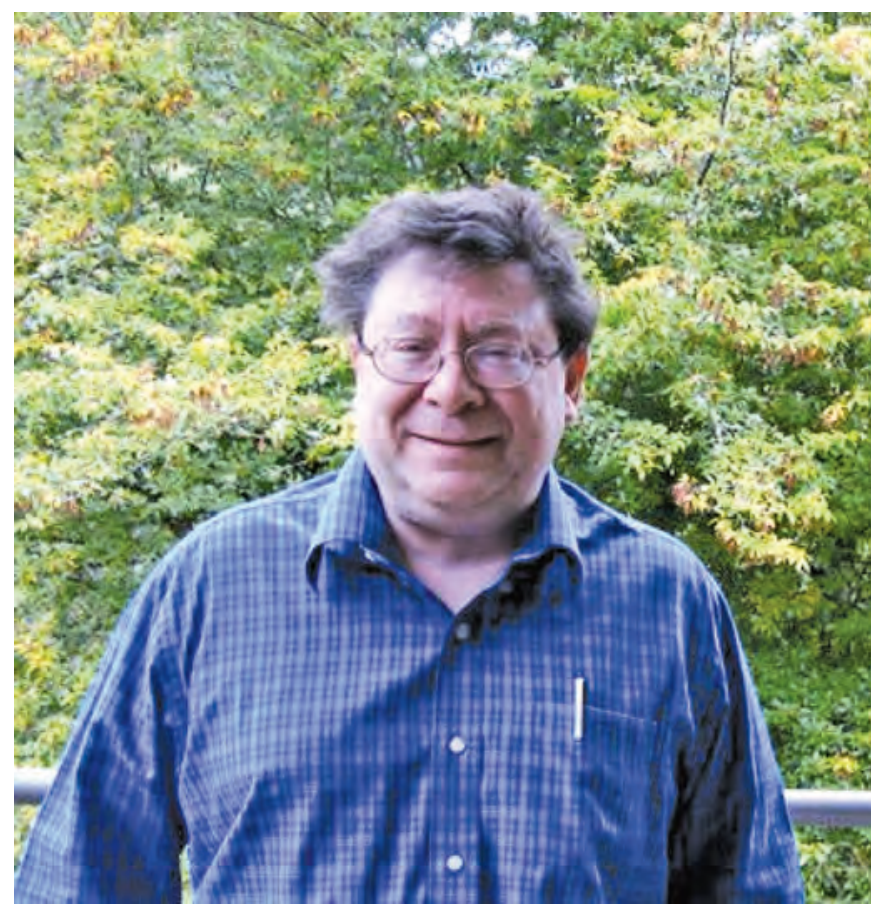

Figure 1. Harvey Friedman proposed the program of reverse mathematics at the 1974 International Congress of Mathematicians.

of Choice is equivalent to Tychonoff's theorem over Zermelo-Fraenkel set theory.

Reverse mathematics studies the strength of everyday mathematical theorems in this way. At the 1974 International Congress of Mathematicians Harvey Friedman [1] (see Figure 1) laid out the founding vision for this program: "When a theorem is proved from the right axioms, the axioms can be proved from the theorem." The key to this analysis is to choose a base system strong enough to formalize the theorems we want to study but not so strong that it proves those theorems outright. Friedman proposed using specific base theories from second-order arithmetic instead of geometry or set theory. Equally importantly, he proposed looking at fundamental theorems of mathematics - results such as the Bolzano-Weierstrass theorem in calculus-rather than more esoteric theorems in set theory or topology.

Stephen Simpson [3] (Figure 2) rephrased the main question as: "Which set existence axioms are required to prove the theorems of everyday, non-set-theoretic mathematics?" In many cases, the required axioms turn out to be very modest.

\section{Second-Order Arithmetic}

Second-order arithmetic is a family of formal systems for studying the natural numbers, real numbers, and many other basic mathematical objects. It takes as given only two fundamental types of objects: "numbers," which are intended to represent natural numbers, and "sets," which are intended to represent sets of natural numbers.
Work of Weyl, Hilbert and Bernays, and Feferman showed that much of elementary real analysis can be studied in second-order arithmetic via arithmetization, in which more complicated objects are represented-coded-as numbers or sets of numbers.

For example, an integer can be coded as a pair of natural numbers with the correct difference, and a rational number can be coded as a pair of integers with the correct ratio. A real number can be coded as a Cauchy sequence of rational numbers, perhaps

Which axioms are required to prove the theorems of everyday mathematics? with a fixed rate of convergence. By using more complex coding systems, we can also represent $\mathbb{R}^{n}$ for each $n$, open subsets of $\mathbb{R}$, and continuous functions from $\mathbb{R}^{n}$ to $\mathbb{R}^{m}$. Algebraic structures such as countable groups and fields and countable vector spaces over countable fields can also be coded into sets of natural numbers. In combinatorics, countable graphs and countable partitions of countable sets can be formalized directly into second-order arithmetic.

Not everything can be coded this way. Second-order arithmetic is not able to talk about arbitrary subsets of $\mathbb{R}$ nor about objects of very high cardinality. In this way, it is more suitable for "ordinary" theorems that talk about countable algebraic objects or complete separable metric spaces.

There are several motivations for using second-order arithmetic. It is a concrete and relatively weak foundational system, and the provability of ordinary mathematical theorems in this setting shows that stronger systems such as set theory or topos theory are not required for

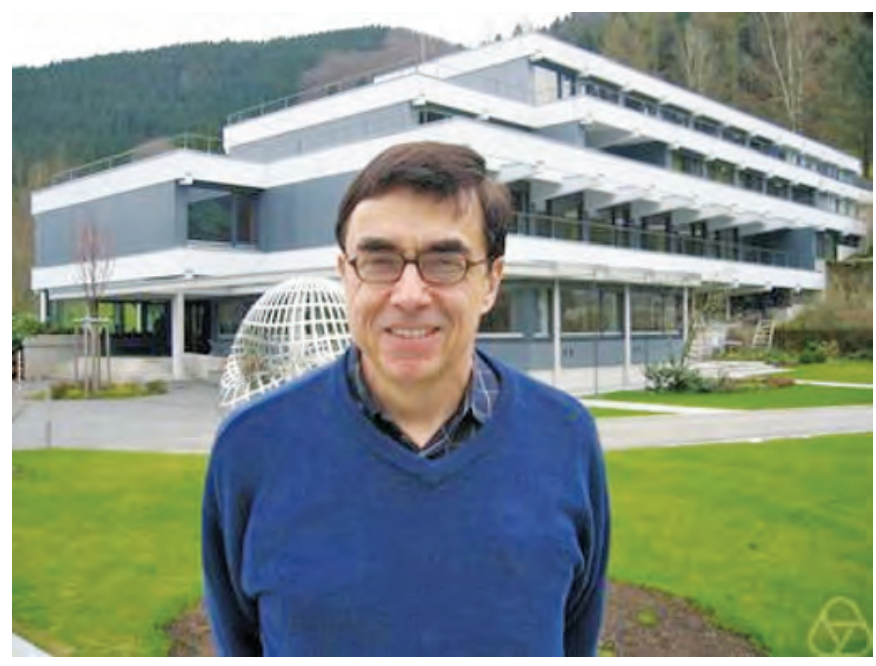

Figure 2. Stephen Simpson has proved many key results in reverse mathematics. His monograph Subsystems of Second Order Arithmetic [3] is the standard graduate-level reference text. 
these theorems. Another motivation is the close relationship between second-order arithmetic and computability theory. This relationship is one of the keys to the success of reverse mathematics: by working in second-order arithmetic, we can use a powerful toolbox of methods from computability theory to study theorems that, at first glance, seem unrelated to computation.

\section{Subsystems of Second-Order Arithmetic}

Subsystems of second-order arithmetic are simply axiom systems for second-order arithmetic which can have varying levels of strength. Following tradition, subsystems are often named with short acronyms, many of which have subscripts or superscripts indicating particular variations. The weakest subsystem usually encountered is known as $\mathrm{RCA}_{0}$. It has axioms saying that $\mathbb{N}$ is a discrete ordered semiring and a set of relatively weak induction axioms. $\mathrm{RCA}_{0}$ also has set existence axioms which say, essentially, that if we have sets $B_{1}, \ldots, B_{k}$ and a set $A$ is Turing computable from these sets, then the set $A$ must exist. The acronym "RCA" stands for "recursive comprehension axiom," where "recursive" is used as a synonym of "computable."

A model $M$ of $\mathrm{RCA}_{0}$ consists of a set of numbers $\mathbb{N}^{M}$, which may or may not be the ordinary natural numbers, and a collection of subsets of $\mathbb{N}^{M}$. Crucially, we do not require that all subsets of $\mathbb{N}^{M}$ must be included. Instead, we rely on the set existence axioms to know that particular sets will be included in the model, that is, to know they will "exist." Allowing the model to contain only some subsets of $\mathbb{N}^{M}$ also avoids the standard proof that all models of Peano's axioms are isomorphic, because that proof requires quantifying over all subsets.

The standard model of second-order arithmetic consists of the ordinary natural numbers and every subset of the natural numbers. Another important model of $\mathrm{RCA}_{0}$ has the ordinary natural numbers but only includes the Turing computable subsets of the naturals. We say that this model, called REC, "believes that every set is computable." If a theorem is provable in $\mathrm{RCA}_{0}$, then the theorem is true in REC. Thus, in particular, a theorem provable in $\mathrm{RCA}_{0}$ cannot imply the existence of uncomputable sets of natural numbers. We often view $\mathrm{RCA}_{0}$ as a formalization of computable analysis.

The remaining subsystems usually encountered in reverse mathematics (see Figure 3 ) consist of $\mathrm{RCA}_{0}$ together with additional axioms. One such subsystem, $\mathrm{ACA}_{0}$, consists of $\mathrm{RCA}_{0}$ with one additional axiom: "if $f: \mathbb{N} \rightarrow \mathbb{N}$ is a function, the range of $f$ must exist." This seemingly innocuous statement is not provable in $\mathrm{RCA}_{0}$, as it is not true in REC. In terms of computability, the new axiom is equivalent to saying that for each set $A \subseteq \mathbb{N}$ we may form the Turing jump $A^{\prime}$. (The Turing jump of a set is the range of a particular function computable from the set.) Thus, in a model of $\mathrm{ACA}_{0}$, the collection of sets is closed under Turing jump. We often view $\mathrm{ACA}_{0}$ as a formalization of Weyl's predicative analysis. The acronym "ACA" stands for "arithmetical comprehension axiom."

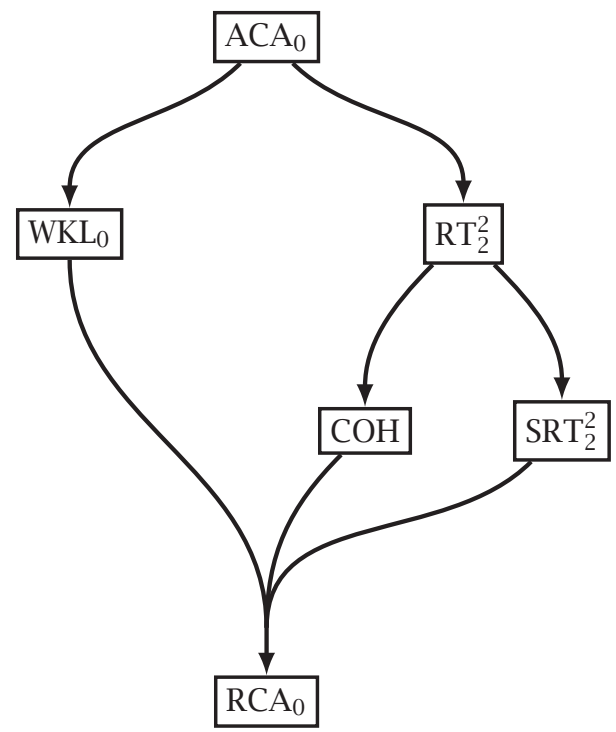

Figure 3. Relationships between six subsystems. $W K L_{0}$ and $\mathrm{RT}_{2}^{2}$ form an incomparable pair below $\mathrm{ACA}_{0}$. $\mathrm{RT}_{2}^{2}$ itself can be split into two strictly weaker systems, $\mathrm{COH}$ and $\mathrm{SRT}_{2}^{2}$, which together are equivalent to $\mathrm{RT}_{2}^{2}$.

There are many subsystems between $\mathrm{RCA}_{0}$ and $\mathrm{ACA}_{0}$. One of these, $\mathrm{WKL}_{0}$, is named after a weak form of König's lemma and is related to several theorems of analysis and countable algebra. A second, $\mathrm{RT}_{2}^{2}$, is a fragment of the infinite version of Ramsey's theorem. These systems are incomparable: neither proves the axioms of the other over $\mathrm{RCA}_{0}$. Moreover, $\mathrm{RT}_{2}^{2}$ can be split into a "cohesive set" principle $\mathrm{COH}$ and a "stable version" $\mathrm{SRT}_{2}^{2}$. Each of these is strictly weaker than $\mathrm{RT}_{2}^{2}$, but taken together their axioms are equivalent to the axiom of $\mathrm{RT}_{2}^{2}$ over $\mathrm{RCA}_{0}$. Figure 3 shows the relationship between the subsystems just mentioned.

The measure of strength in reverse mathematics gives a complex, nonlinear hierarchy. However, many ordinary theorems of mathematics turn out to be equivalent, over $\mathrm{RCA}_{0}$, to the axioms of one of five linearly ordered subsystems (the reason for this phenomenon is not completely clear). Simpson named these subsystems the "Big Five" (see Figure 4).

The weakest three of the Big Five are $\mathrm{RCA}_{0}, \mathrm{WKL}_{0}$, and $\mathrm{ACA}_{0}$. These three are sufficient for almost all theorems of a standard undergraduate mathematics curriculum that can be stated in second-order arithmetic. Beyond them are two stronger systems, $\mathrm{ATR}_{0}$ and $\Pi_{1}^{1}-\mathrm{CA}_{0}$. The Big Five subsystems are shown in Figure 4 along with a mathematical theorem representative of each.

\section{Stillwell's Reverse Mathematics}

Stillwell focuses on $\mathrm{RCA}_{0}, \mathrm{WKL}_{0}, \mathrm{ACA}_{0}$, and on a few carefully chosen mathematical results from introductory real analysis. He carefully limits the amount of logic and computability included in the main portion of the text. 


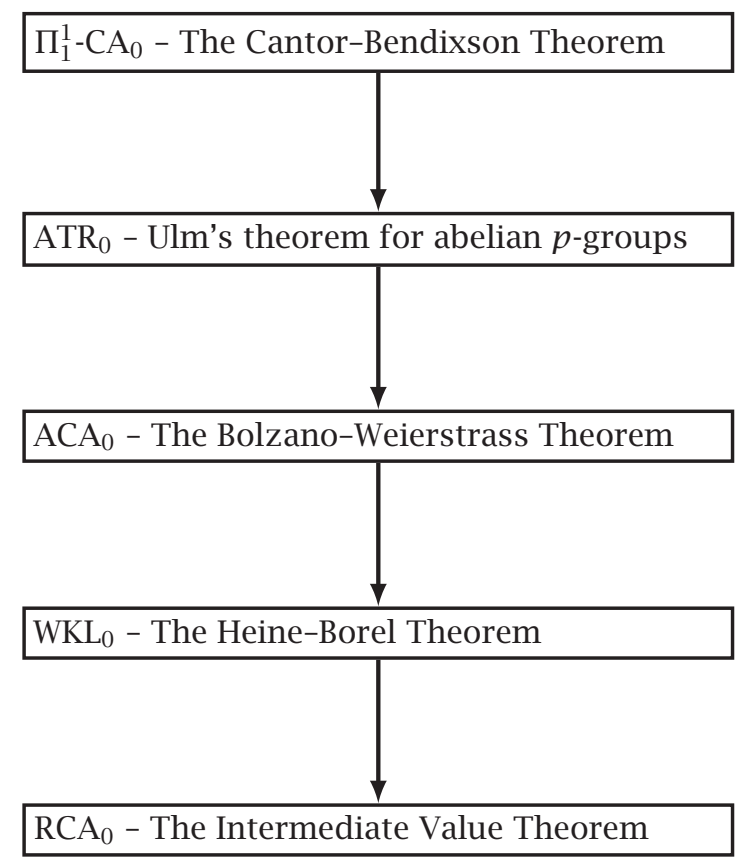

Figure 4. The "Big Five" subsystems of second-order arithmetic and a representative theorem equivalent to each.

The later portion includes some comments on the logical details, but still at a level intended for a general reader. This necessarily means that some of the beautiful interactions between computability, second-order arithmetic, and mathematical theorems are less clearly visible, but it is very much in line with the mathematical character of reverse mathematics.

A key aspect of reverse mathematics, which can sometimes be obscured by the logical methods employed, is an underlying love of the mathematics being studied. Many researchers in reverse mathematics gravitate towards well-known, basic theorems of mathematics (the BolzanoWeierstrass theorem, Ramsey's theorem, Hilbert's basis theorem, etc.) in order to understand these theorems better. The mathematics itself provides a central motivation for the logical analysis being performed.

Accordingly, work in reverse mathematics requires a detailed understanding of both the logical tools and the mathematics being studied. More advanced treatments may make substantial assumptions about the reader's background in both of these areas. Stillwell presents a careful introduction to a portion of elementary real analysis, its arithmetization, and its provability in $\mathrm{ACA}_{0}$ without assuming the reader is already fluent in the details.

The standard proof of a result can sometimes be formalized directly into a desired subsystem of secondorder arithmetic. In other cases, a new proof must be discovered if the previous proof used methods that were stronger than necessary. In either case, the reverse mathematics analysis of a theorem leaves us with more information than the mere fact that the theorem is provable. Stillwell's treatment of several theorems of elementary real analysis demonstrates this in a way unobscured by the underlying logical machinery.

Stillwell shows how $\mathrm{ACA}_{0}$ is equivalent over $\mathrm{RCA}_{0}$ to the Bolzano-Weierstrass theorem ("every bounded sequence of reals has a convergent subsequence"). He also proves that $\mathrm{WKL}_{0}$ is equivalent over $\mathrm{RCA}_{0}$ to the Heine-Borel theorem in the form "if a sequence of rational intervals covers $[0,1]$, then some finite subsequence is also a cover." Along the way, he clearly demonstrates how these results are related to particular statements about infinite paths through trees, which is what allows computability methods to be applied to the theorems.

In Zermelo-Fraenkel set theory, the BolzanoWeierstrass theorem and the Heine-Borel theorem are both provable, and so they are trivially equivalent. By looking at them in a weaker base system such as $\mathrm{RCA}_{0}$, we see that there is, in a precise sense, a difference between open cover compactness and sequential compactness on the real line. This kind of separation result illustrates the additional information that can be obtained through reverse mathematics. We understand the two theorems better by seeing an intrinsic way in which they differ.

Because the author avoids most of the logical machinery, the book will not prepare readers to jump directly into research. The introductory treatment also omits subsystems stronger than $\mathrm{ACA}_{0}$, which are required to prove theorems such as the Cantor-Bendixson theorem. These higher subsystems are closely related to generalized computability theory in much the same way that the weaker systems are related to Turing computability. So, if it does its job, the book will leave a reader asking for more.

What could be the next step after reading this book? The first four chapters of Simpson [3] give a thorough survey of arithmetization and the reverse mathematics of mathematical theorems in $\mathrm{RCA}_{0}, \mathrm{WKL}_{0}$, and $\mathrm{ACA}_{0}$. Hirschfeldt [2] provides an introduction to many recent results in the reverse mathematics of combinatorics. Solomon [4] gives an introductory, but more technical, summary of several reverse mathematics results in algebra. Beyond these is a large and growing research literature.

\section{References}

[1] HARVEY FRIEDMAN, Some systems of second order arithmetic and their use, Proc. Intl. Cong. Math., (Vancouver, B. C., 1974), Canad. Math. Congress, 1975, pp. 235-242. MR0429508

[2] Denis R. HiRsCHFELDT, Slicing the Truth, Lecture Notes Series., Inst. Math. Sci., Nat. Univ. of Singapore, vol. 28, World Scientific, 2015. MR3244278

[3] STEPHen G. Simpson, Subsystems of Second Order Arithmetic, 2nd ed., Perspectives in Logic, Cambridge Univ. Press, Assoc. Symb. Logic, 2009. MR2517689

[4] ReED Solomon, Ordered groups: a case study in reverse mathematics, Bull. Symbolic Logic 5 (1999), no. 1, 45-58. MR1681895

[5] JOHN STILLWELL, Reverse Mathematics: Proofs from the Inside Out, Princeton Univ. Press, 2017. 


\section{samsi}

The Statistical and Applied Mathematical Sciences Institute (SAMSI) is soliciting applications from statistical, mathematical and computational scientists for up to 6 postdoctoral positions for the SAMSI research programs in 2019-2020. The three programs are:

(1) Games, Decisions, Risk and Reliability, (2) Deep Learning, and

(3) Causal Inference. Appointments will begin in August, 2019 and typically run for two years, although they can also be arranged for a single year. Appointments are made jointly between SAMSI and one of its partner universities (Duke University, North Carolina State University, and the University of North Carolina Chapel Hill). Teaching opportunities may be available. The positions offer extremely competitive salaries, a travel stipend, and health insurance benefits.

Criteria for selection of SAMSI Postdoctoral Fellows include demonstrated research ability in statistics, applied mathematics, and/or computational science, excellent computing skills, and the ability to communicate both orally and in writing. Also, the preferred applicant will have a strong interest in one or more of the research programs scheduled for 2019-2020. The deadline for full consideration is December 15, 2018, although later applications will be considered as resources permit.

Please specify which SAMSI programs you are applying for in your cover letter and why you believe you would be a good fit for those programs.

To apply, go to mathjobs.org:

SAMSIPD2019 Job \#12160

To see more about these programs, visit:

www.samsi.info/games-19-20

www.samsi.info/sem-deep-Ing

www.samsi.info/sem-cas-inf

${ }^{* *}$ SAMSI is an Equal Opportunity employer**

\section{Photo Credits}

Figure 1 courtesy of Renate Schmid, 2011, Oberwolfach Photo Collection.

Figure 2 courtesy of Renate Schmid, 2008, Oberwolfach Photo Collection.

Figures 3 and 4 created by author.

Author photo courtesy of Rick Haye / Marshall University.

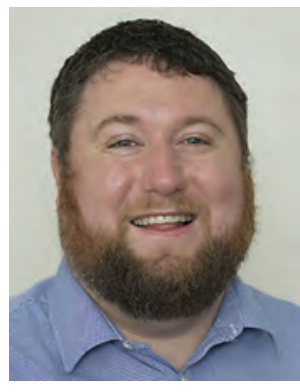

Carl Mummert

\section{ABOUT THE AUTHOR}

Carl Mummert's research is in mathematical logic (particularly reverse mathematics, second-order arithmetic, and computability theory), topology, and combinatorics. His teaching incorporates techniques from Inquiry Based Learning (IBL). 
Youn donation to the

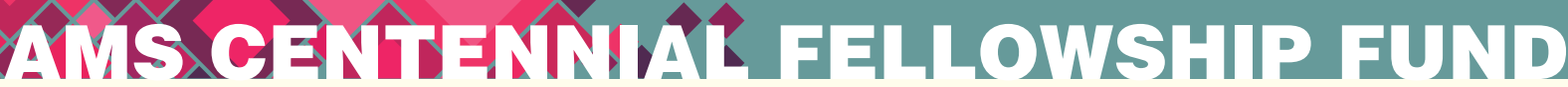
advances mathematics for many.

Recipients of this one-year mathematics research fellowship have contributed substantially to their areas of research, furthering the mathematical sciences.

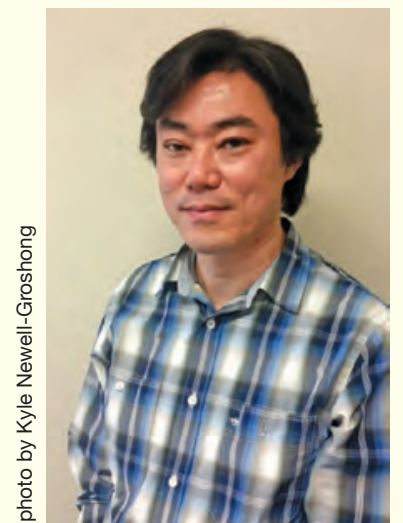

2017-2018 Centennial Fellowship recipient Shuichiro Takeda Associate Professor of Mathematics at University of Missouri. Research interests include automorphic forms and representations of $p$-adic groups, especially from the point of view of the Langlands program
Since 1974, _ scholars have been able to further their area of research with help from the Centennial Fellowship (formerly named Research Fellowship).
a. 43
b. 50
c. 75
d. 106

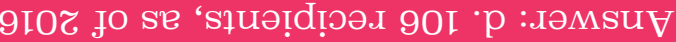

To donate to the Centennial Fellowship, visit www.ams.org/support 


\title{
Interview with NSF Division of
}

\section{Mathematical Sciences Director}

\section{Juan Meza}

\author{
by Robert L. Bryant
}

\author{
Communicated by Harriet Pollatsek
}

In February 2018 Juan Meza became director for the Division of Mathematical Sciences (DMS) at the NSF. He came to NSF from University of California Merced where he served most recently as the dean of Natural Sciences. Prior to joining UC Merced, Meza served as department head and senior scientist for high performance computing research at Lawrence Berkeley National Laboratory. Meza received the 2013 Rice University outstanding engineering alumni award, was the 2008 recipient of the Blackwell-Tapia Prize

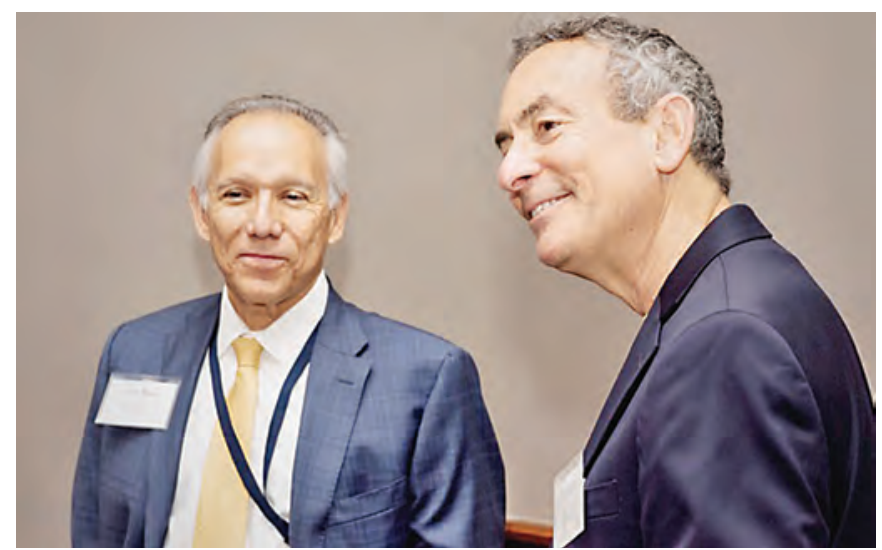

Figure 1. DMS Director Juan Meza (left) and AMS President Ken Ribet at the AMS-Congressional briefing talk given by Eric Demaine in May 2018.

Robert L. Bryant is the Philip Griffiths Professor of Mathematics at Duke University and former president of the AMS. His email address is bryant@math.duke.edu.

For permission to reprint this article, please contact: reprint-permission@ams .org.

DOI: http://dx.doi.org/10.1090/noti1731 and the SACNAS Distinguished Scientist Award, and is a Fellow of the AAAS. He has served on numerous boards, including the National Research Council Board on Mathematical Sciences and their Applications, and the NSF Mathematical and Physical Sciences Advisory Committee. This interview was conducted via email.

You've had extensive administrative experience, both in leading scientific teams and more broadly in the University of California system. Could you tell us something about this and what stimulated your interest in serving as director of NSF's Division of Mathematical Sciences (DMS)?

Yes, I first became a manager in 1997 while I was at Sandia National Labs, but I was leading small research projects before that as well. I remember thinking hard about whether I wanted to go into management because I was having so much fun doing research. So, I decided to ask a lot of people whom I respected about their experiences. What I learned from them was that, if you do it well, you are able to accomplish a lot more through other people than you could do on your own. I've tried to follow their advice although it's sometimes easier said than done. I then moved to Berkeley Lab, followed by a position as dean for the School of Natural Sciences at Merced. The most important lesson that I've learned is that each organization has a distinct culture that you need to respect and work with, and your leadership style needs to adapt if you want to be successful.

That leads me to your question as to what stimulated my interest in DMS, and it's partly what I learned early on-the possibility of doing something that would have a larger impact. Another strong motivator is that the mathematics community has been really good to me over the years and I felt that it was time to pay it forward. It 
sounds a little old-fashioned, but I grew up in the John F. Kennedy generation when you were told-"Ask not what your country can do for you; ask what you can do for your country." As a result, service to the community has always been a guiding principle for me.

What are some of the unique aspects and challenges of the role of Director of DMS?

I suspect that it's still a little early for me to give you a good response to this question, but at this point in time, what I've learned is that one of the most important roles is to act as the go-between between the mathematics and statistics communities and the rest of the world, including within NSF as well as outside. I have found over the years that this can be a challenge, but it's also one of the most interesting and fun aspects of the job because I get to learn about all the cutting-edge mathematics and then I have a chance to try to explain the beauty, the importance, and especially the value of the research to others who may not have the expertise or time to learn about it.

Most of us who have been supported by the DMS in one way or the other (a large fraction of the US mathematical community) have ideas about what the DMS could or should be addressing with its resources. Are there some particular issues that you have in mind to look into or work on during your tenure?

I have some general ideas, but I don't have anything specific just yet. In my first three months, I've been trying to speak to as many people and constituencies as I can find time for. My first impression is that DMS has been doing a great job in providing support to the community, and the research it funds has had a great impact in many different areas. As a result, I think it is important to ensure that I don't change anything that is already working well.

However, as I learn more about what we're currently funding and the needs of the community, I hope to be able to provide everybody with a clearer sense of where I think DMS might want to head. In fact, I hope this article encourages the math community to reach out to me and send me their best ideas.

Increasing participation of under-represented minorities in the STEM workforce has long been one of NSF's priorities. Would you say something about your own experience with involvement in this effort, perspective on effective strategies, and how the DMS director might help further this goal?

As you know, that has been a priority of mine for a long time and I hope to be able to contribute to this area. I'll also expand your question to include gender diversity as that is equally important in our fields. My own experiences have been shaped by several mentors who have helped me throughout my career as well as some lucky breaks I've had at key points in my career. From those experiences, I learned two important lessons. The first is that mentors play a critical role, especially those that come from similar backgrounds to yourself. The second is the need to recognize that this is a shared responsibility and that we can't place all of our expectations on women and underrepresented minorities to solve this problem. We all have a role to play in addressing this national challenge.
When I was dean at UC Merced, the accomplishment that I was the proudest of was in our faculty hiring. Overall, we hired 38 new faculty members of whom 18 were women and 7 were underrepresented minorities. In the Applied Mathematics department alone, we had a 50-50 ratio of men and women.

You've had considerable experience with interdisciplinary research, working in academia, national laboratories, and industry. Can you say something about what you would like to work on at NSF to help promote collaboration between the "traditional" DMS researcher and those supported by other NSF directorates?

Interdisciplinary research is a key area and one that has seen large growth in recent years. My own experiences have taught me that the intersection between different fields can provide some of the richest sources of interesting mathematical problems. As I mentioned earlier, academia, national labs, and industry all have their own individual cultures, which causes them to address problems in different ways. And that's not including the differences between individual scientific disciplines.

One lesson I've taken away from my own work is that you need to listen carefully to the other side and be willing to ask what may appear as really naïve questions. At DMS, I hope to be able to help bridge those different cultures so that we can start conversations about how to tackle some of what I see as the most challenging problems because they require expertise from different disciplines. However, let me also state that it is critically important to maintain a strong base program in the individual areas because this feeds into the interdisciplinary programs. The core programs are a necessary component for interdisciplinary programs to be successful.

Some areas where DMS PIs [Principal Investigators] could participate with researchers in other directorates are the NSF's Ten Big Ideas. These are new priority areas from Director Cordova, and the whole foundation has been working hard to define the most pressing research problems in each of these areas. We are already funding projects in several of these areas, but I hope that the math community sees the opportunity for even more engagement. From what I've seen, there are interesting new mathematical approaches one might use in many of the Big Ideas. (See sidebar on the Big Ideas.)

The NSF would like to promote partnerships with industry and other supporters of basic scientific research. What do you see as the challenges and opportunities of this kind of "fundraising" for scientific research?

Great question, as it gives me the opportunity to highlight a new program that DMS will start this year. It's a wonderful collaboration between NSF and the Simons Foundation to support new centers for the study of complex biological systems. This not only emphasizes the last point about the importance of interdisciplinary research but also points to the wonderful opportunities that arise when you team up with other supporters of basic research like the Simons Foundation.

The goal of the centers will be to bring together teams of biologists and mathematicians to address some of the 


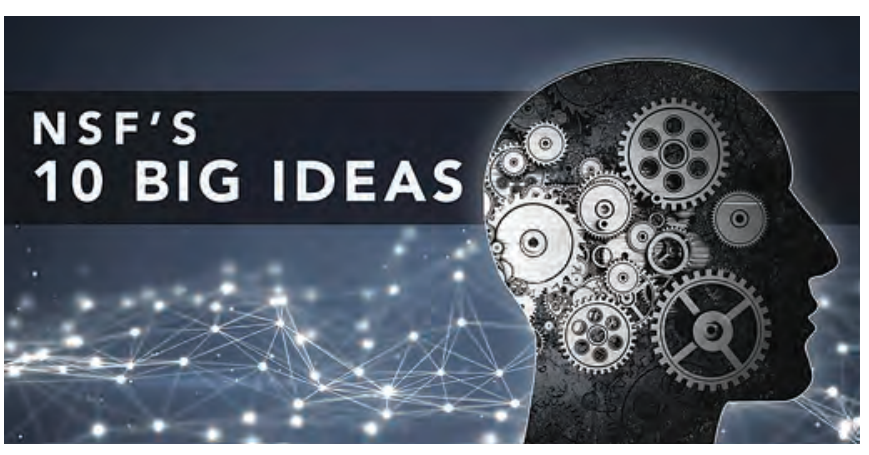

In 2016, NSF unveiled a set of "Big Ideas"-10 bold, long-term research and process ideas that identify areas for future investment at the frontiers of science and engineering. With its broad portfolio of investments, NSF is uniquely suited to advance this set of cutting-edge research agendas and processes that will require collaborations with industry, private foundations, other agencies, science academies and societies, universities and the education sector. The Big Ideas represent unique opportunities to position our nation at the cutting edge-indeed to define that cutting edge-of global science and engineering leadership and to invest in basic research and processes that advance the United States' prosperity, security, health, and well-being.

1. Harnessing the Data Revolution

2. The Future of Work

3. Navigating the New Arctic

4. Multi-Messenger Astrophysics

5. The Quantum Leap

6. Understanding the Rules of Life

7. Mid-Scale Research Infrastructure

8. NSF 2026

9. Growing Convergence Research at NSF

10. NSF INCLUDES

In September the Joint Policy Board for Mathematics, consisting of AMS, ASA, MAA, and SIAM, issued the following statement on Mathematics and Statistics Community Engagement with NSF Big Ideas:

It is critically important that mathematical scientists engage in the conversations that the NSF Big Ideas have launched, whether by participating in upcoming workshops on Big Ideas topics, responding to special funding solicitations, or by communicating key research needs to NSF. We encourage all mathematical and statistical scientists to become familiar with the Big Ideas, and to contribute in a substantial way to the NSF's research agenda.

rittps://www. cbmsweb .org/2017/09/jpbm -statement-nsf-big-ideas most challenging problems in biology today. For example, how does a genotype lead to all the various phenotypes that we see in real life? This is still a poorly understood area-we hope that applying mathematical models and techniques and working closely with biologists will help us better understand these processes. It's also a wonderful opportunity to train the next generation of biologists and mathematicians in ways that I think will fundamentally change the way we understand biology.

Image Credits

Figure 1 by Scavone Photography.

Author photo (c) Duke University.

NSF 10 Big Ideas from https://www.nsf.gov/news/special_ reports/big_ideas

\section{ABOUT THE INTERVIEWER}

Robert L. Bryant is a differential geometer whose research applies the theory of exterior differential systems to a variety of problems in geometry and partial differential equations. He has served as director of the Mathematical Sciences Research Institute (2007-2013) and as the president of the American

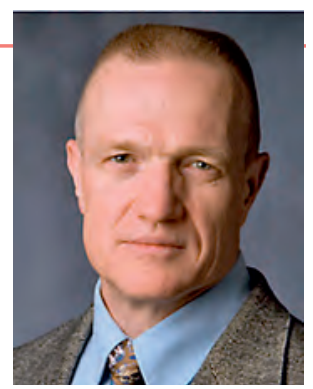
Robert L. Bryant
EDITOR'SNOTE.DMS presentations are now posted online at https://www.nsf.gov/mps/dms/presentations .jsp. 


\section{The June/July Contest Winner Is...}

Robert Cohen, who receives our book award.

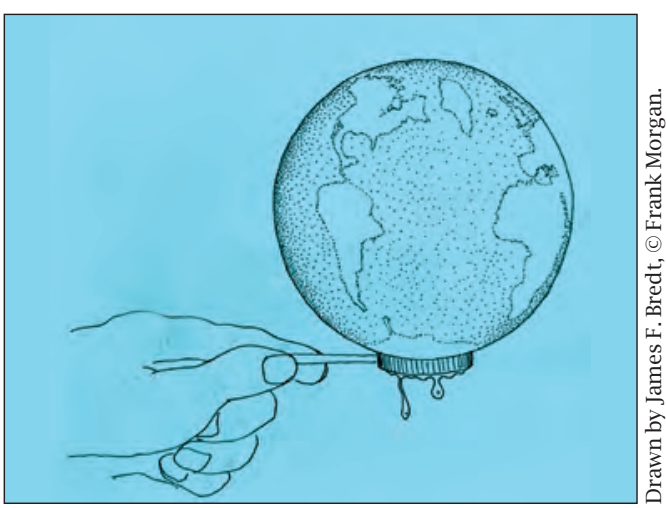

Handle with Care!

\section{The October Caption Contest:} What's the Caption?

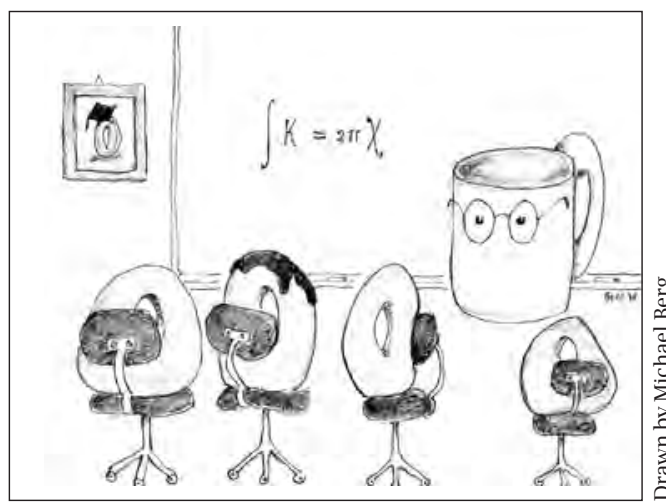

Submit your entry to captions@ams.org by October 25 . The winning entry will be posted here in the December 2018 issue.
"What has been affirmed without proof can also be denied without proof." —Euclid

\section{Meteorologist}

OUR FORECAST SAYS THERE'S A 20\% CHANCE OF RAIN FOR EACH OF THE NEXT FIVE HOURS. HOU LIKELY IS IT TO RAIN THIS AFTERNOON? IT'S A SIMTPLE QUESTION, BUT I DON'T KNOWJ THE ANSWER. ISEACH HOUR INDEPENDENT? CORRELATED? OR IS RAIN GUARANTEED AND WE'RE JUST UNSURE OF THE TIMING?

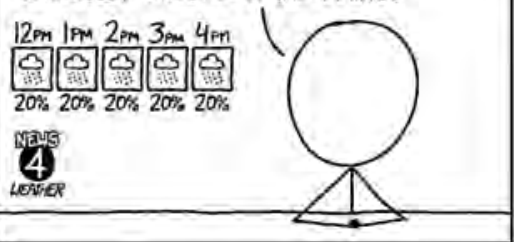

IT SAYS "SCATTERED SHOUERS." IS THIS THE CHANCE OF RAIN SOMEZWHERE IN YOUR AREA? HOW BIG IS YOUR AREA? WHAT IF YOU HANE TWO LOCATIONS YOU'RE WORRIED ABOUT? I'VE ASKED MANAGEMENT, BUT THEYVE STOPPED ANSLIERING MY EMAILS, SO-HANG ON, THE SECURITY GUY IS COMING OVER.

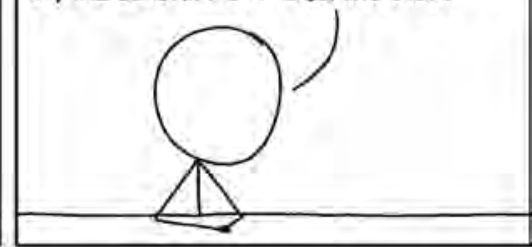

TEOMICAL DIFFICULTIES

WE APOLOGIZE FOR HIRNG

A METEOROLGST WITHA

PURE MATH BACKGROUND.

LEUL BE BACKON

THE AIR SHORTLY.

NEIS

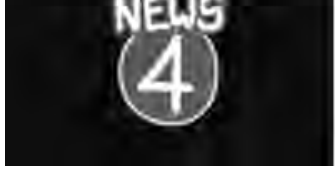

SORRY ABOUT THAT. HII I'M VOUR NEW METEOROLGIST:

AND YOU'RE NOT A MATHEMATKCIAN, RIGT?

NO. I DO HAVE A LINGUISTICS DEGREE.

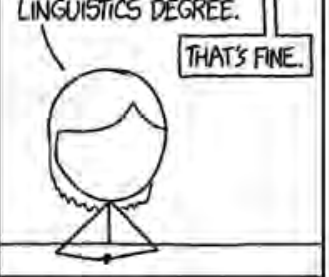

IT MIGHT RAIN THIS AFTERNOON. QUT WHAT 15 " "IT" HERE? ISIT A TRUE DUMTYY PRONOUN, AS IN THE PHRASE "IT'S TOO BAD?" OR IS THE WEATHER AN ENTITY? ALSO, WHAT IF I SAY "ITS HOT OUT, AND GETTING BIGGER?"

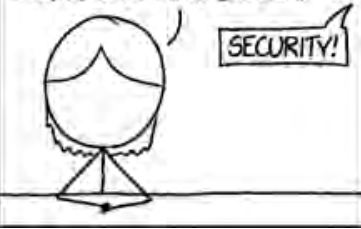

See https://xkcd.com/1985/, licensed under a Creative Commons Attribution-NonCommercial 2.5 License. 


\section{Report of the Treasurer (2017)}

\section{Introduction}

The fiscal year 2017 was one of significant change for the American Mathematical Society (AMS) due to the acquisition of the MAA Press in the last quarter of the year. Even so, the AMS finished the 2017 fiscal year with an operating bottom line of about $\$ 1,000,000$. This is lower than recent fiscal years, but due to investment market performance, unrestricted net assets increased by more than $\$ 19$ million. The Treasurer is pleased to report that overall, it was a financially healthy year for the AMS.

The Report of the Treasurer is presented in the Notices annually. Its purpose is to discuss the financial condition of the Society as of the immediately preceding fiscal yearend and the results of its operations for the year then ended. One of the key responsibilities of the Treasurer is to lead the Board of Trustees in the oversight of financial activities of the Society. This is done through close contact with the executive staff of the Society, review of internally generated financial reports, review of audited financial statements, and twice yearly meetings with the Society's independent auditors. Through these and other means, the Trustees gain an understanding of the finances of the Society and the important issues surrounding its financial reporting.

When reviewing the financial results of the AMS presented here, it is important to note that the financial support for its membership and professional programs is derived from multiple sources. First, a board-designated endowment fund, the Operations Support Fund (OSF), provided $\$ 2,831,000$ in operating support to the membership and professional programs in 2017. The OSF is a fund that has grown throughout the years from operating net income as well as investment gains; because the fund is dependent upon market conditions, the amount provided varies from year to year. In addition, the membership and professional programs are supported through dues income and contributions.
Finally, the margin from the publication programs supports these services as well. Without the margin from both publications and the OSF income, dues and contributions alone would not provide enough support to continue professional programs, such as MathJobs.Org, scholarships, fellowships, and Notices.

The Society segregates its net assets, and the activities that increase or decrease net assets, into three types: unrestricted, temporarily restricted, and permanently restricted. Total net assets at the end of 2017 were approximately $\$ 163$ million. Unrestricted net assets are those that have no requirements as to their use placed on them by donors outside the Society. A substantial majority of the Society's net assets are in this category. Temporarily restricted net assets are those with donor-imposed restrictions or conditions that will lapse upon the passage of time or the accomplishment of a specified goal. Examples of the Society's temporarily restricted net assets and related activities include grant awards and the spendable income from prize and other income-restricted endowment funds. Permanently restricted net assets are those that must be invested in perpetuity with the income spent only on specific programs and prizes for which the donations were intended, and are commonly referred to as endowment funds. The accompanying financial information principally relates to the unrestricted net assets, as this category

\section{Key Operating Results (in 000's)}

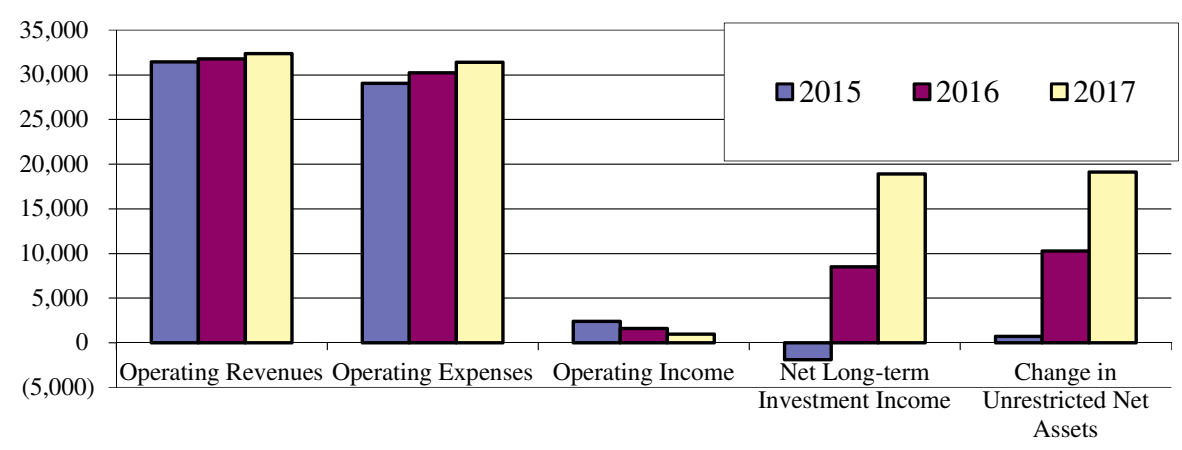

Figure 1

All currency discussed in this report refers to US dollars.

"k" denotes thousand.

"m" denotes million. 
includes the operating activities of the Society.

\section{Operating Results}

Apart from the low investment returns in 2015, Figure 1 shows that the past three years have been good years, financially, for the Society. Figure 1 also illustrates how important the unrestricted, long-term investments are to the financial health of the organization, as the returns on the investments are generally much higher than the operating income. These unrestricted investment returns amounted to $\$ 18,901,234$ in 2017.

There were two financial transactions that occurred at the Society in 2017 that contributed to a lower than normal operating income as shown in Figure 2. As part of the strategic plan initiative to publish more mathematics, the AMS acquired the MAA Press at the end of September from the Mathematical Association of America. Also, the internal accounting for AMS Points, which are exchangeable for AMS books and services, and are awarded to mathematicians for reviewing and editorial work, changed in 2017; we are now required to count them as an expense in the year they are awarded. This is referred to as an accounting accrual and it increased the expenses by about $\$ 554,000$. This accrual will not affect the financial statements as much in future years.

In 2017, the Society's revenues increased by $1.8 \%$ over 2016 revenues. There was a sharp decline in development donations from 2016 to 2017 due to a large bequest the AMS received in 2016, which masks the fact that revenues

\section{AMS Net Operating Income 1996 - 2017 (in 000's)}

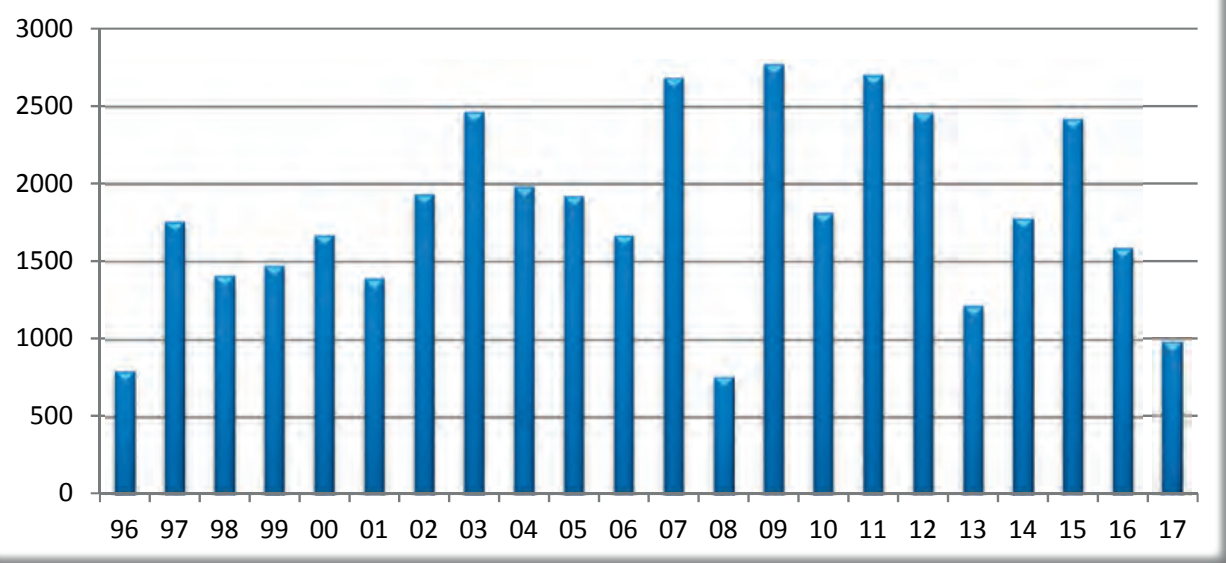

Figure 2

overall were up by about $4 \%$ after subtracting the difference in donations. Publishing revenues did especially well with electronic book backlist and MathSciNet sales providing the bulk of the increase from one year to the next. However, journal subscriptions sales continue to decline due to subscription attrition and the movement of subscribers to less expensive subscription models, such as electronic only or consortia.

In 2017, expenses increased by $4 \%$ over the prior year. Wage and benefit costs increased about 3.5\% due to rapidly increasing health insurance benefits and annual raises for employees. Major contributing factors to increased costs were the aforementioned accrual of AMS Points values and the acquisition of MAA Press. Many costs, such as building, postage and equipment remained the same as the prior year. Figure 3 shows the amounts spent on major expense categories in 2017.

For more detailed information regarding the Society's operating results, please see the financial statements, including the Statements of Activities, located at the end of this report.

\section{Balance Sheets Highlights}

Another report within the financial statements, referred to as the Balance Sheets, is also at the end of this financial review. The Society continues to maintain healthy balance sheets. Total net assets of the organization are $\$ 163$ million, of which \$141 million are unrestricted. Table 1 (see page 1110) shows highlights of the Society's 2017 Balance Sheets.

\section{Figure 3}




\begin{tabular}{|c|c|}
\hline $\begin{array}{l}\text { Highlights of Balance Sheets Changes } \\
\text { from } 2016 \text { to } 2017\end{array}$ & Commentary \\
\hline $\begin{array}{l}\text { Short-term investments decreased by } \$ 1.9 \\
\text { million (-13\%) }\end{array}$ & $\begin{array}{l}\text { This decrease is due primarily to the Society investing in capital } \\
\text { assets including building improvements and the MAA Press. }\end{array}$ \\
\hline $\begin{array}{l}\text { Completed books increased by } \$ 576,000 \\
(42 \%)\end{array}$ & $\begin{array}{l}\text { The book inventory increased mainly because of the acquisition of } \\
\text { MAA Press books. }\end{array}$ \\
\hline $\begin{array}{l}\text { Long-term investments increased by } \$ 22.9 \\
\text { million }\end{array}$ & $\begin{array}{l}\text { The investments increased due to the performance of the investment } \\
\text { markets in } 2017 \text {. The Society's portfolio experiences a } 17.8 \% \text { return. }\end{array}$ \\
\hline $\begin{array}{l}\text { Accounts payable and accrued expenses } \\
\text { increased by } \$ 708,000(17 \%)\end{array}$ & The increase is mostly related to the accrual of the AMS Point values. \\
\hline Deferred Revenue decreased $\$ 668,281(5 \%)$ & $\begin{array}{l}\text { In 2016, approximately } \$ 200,000 \text { in donations reced for purposes } \\
\text { not yet approved by AMS governance were treated as deferred } \\
\text { revenues, but this was reversed in } 2017 \text {. In addition, the number } \\
\text { of prepaid subscriptions dropped at the end of } 2017 \text { compared to } \\
2016 \text {, accounting for the majority of this variance. }\end{array}$ \\
\hline $\begin{array}{l}\text { Postretirement benefit obligation increased } \\
\text { approximately } \$ 600,000(8 \%)\end{array}$ & $\begin{array}{l}\text { This increase was due to a decrease in the interest rate used in the } \\
\text { actuarial calculation of the value of this oblivation. }\end{array}$ \\
\hline
\end{tabular}

Table 1

\section{Statements of Invested Funds}

The Statements of Invested Funds are divided into the permanently restricted funds that have been acquired from donations in the form of endowment funds, and the funds that have been designated by the Board of Trustees for specific purposes (which we refer to as quasi-endowment funds).

The Society's Statements of Invested Funds is a listing of the Society's individual endowment and quasi-endowment funds. In addition, the invested funds of the Society contain a temporarily restricted fund, the Beal Prize, which at year-end amounted to $\$ 1.6$ million. The corpus of this fund, $\$ 1,000,000$, is set aside to fund a prize for solving the Beal Conjecture. The spendable income from the fund supports the Erdos Lecture and other programs. Overall, the 2017 Statements of Invested Funds show an increase of about $\$ 23$ million over the prior year. The investment return was $17.8 \%$. Approximately $\$ 600,000$ in donations were added to the permanent endowment during the year.

The quasi-endowment or board-designated funds are set aside for various purposes. The Economic Stabilization Fund (ESF) is a fund set aside to cover the postretirement benefit obligation and $50 \%$ of the current annual operating expenses in case of disaster. The ESF also contains funds to self-insure against flood risk. The Society's largest quasi-endowment fund, the Operations Support Fund (OSF), valued at approximately $\$ 111.7$ million, provided $\$ 2,831,000$ in spendable income to the AMS operations in 2017.

\section{Summary Financial Information}

The pages that follow contain Statements of Activities and Balance Sheets from the audited financial statements of the AMS; the Statements of Invested Funds are from the internal financial records of the AMS. Any member may contact the AMS to request the full audited statements of the Society. The Treasurer will be happy to answer any questions members may have regarding the financial affairs of the Society.

Respectfully submitted, Jane M. Hawkins AMS Treasurer 


\section{AMERICAN MATHEMATICAL SOCIETY}

\section{Balance Sheets}

\section{Assets}

Cash

Certificates of deposit

Short-term investments

Accounts and contributions receivable, net of allowances of $\$ 272,980$ and $\$ 284,980$ in 2017 and 2016, respectively

Contributions receivable, net

Deferred prepublication costs

Completed books

Prepaid expenses and deposits

Land, buildings and equipment, net

Long-term investments

Intangible assets, net

December 31, 2017

2016

\section{Total assets}

\begin{tabular}{|c|c|c|c|}
\hline & Dece & $n h$ & \\
\hline & 2017 & & 2016 \\
\hline$\$$ & 993,242 & $\$$ & $1,131,379$ \\
\hline & 466,759 & & 660,000 \\
\hline & $12,699,193$ & & $14,600,782$ \\
\hline & 787,938 & & 523,200 \\
\hline & 559,702 & & 30,000 \\
\hline & 600,406 & & 594,587 \\
\hline & $1,937,399$ & & $1,360,939$ \\
\hline & $1,780,182$ & & $1,632,127$ \\
\hline & $5,040,855$ & & $5,086,655$ \\
\hline & $162,999,715$ & & $140,116,402$ \\
\hline & $1,385,235$ & & - \\
\hline$\$$ & $189,250,626$ & $\$$ & $165,736,071$ \\
\hline
\end{tabular}

\section{Liabilities and Net Assets}

Liabilities:

Accounts payable and accrued expenses

Accrued study leave pay

Deferred revenue

Postretirement benefit obligation

\section{Total liabilities}

Net assets:

Unrestricted:

Undesignated

\begin{tabular}{rrr}
$\$ 4,898,847$ & $\$$ & $4,190,837$ \\
860,403 & & 736,298 \\
$12,257,831$ & & $12,926,112$ \\
$8,249,948$ & & $7,646,939$ \\
\cline { 1 - 1 } \cline { 1 - 1 } $\mathbf{2 6 , 2 6 7 , 0 2 9}$ & & $\mathbf{2 5 , 5 0 0 , 1 8 6}$ \\
& &
\end{tabular}

Designated

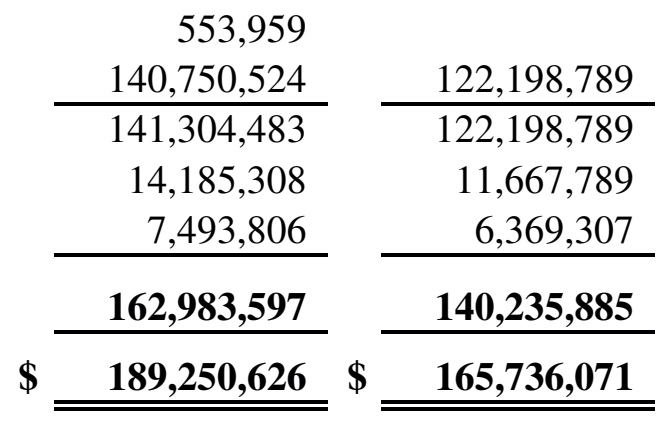

Temporarily restricted

Permanently restricted

Total net assets

Total liabilities and net assets 


\section{FROM THE AMS SECRETARY}

\section{AMERICAN MATHEMATICAL SOCIETY Statements of Activities}

Changes in unrestricted net assets:

Operating revenue, including net assets released from restrictions:

Mathematical reviews

Journals

Books

Dues, services, and outreach

Investment returns appropriated for spending

Other publications-related revenue

Grants, prizes and awards

Meetings

Unrestricted contributions

Short-term investment income

Other

Total operating revenue

Operating expenses:

Mathematical reviews

Journals

Books

Publications indirect

Customer services, warehousing and distribution

Other publications-related expense

Membership, services and outreach

Grants, prizes and awards

Meetings

Governance

Member and professional services indirect

General and administrative

Other

\section{Total operating expenses}

Excess of operating revenue over operating expenses

Nonoperating revenues and expenses:

Investment returns (loss) net of investment returns available for spending

Use of board designated funds from Backfile Digitization,

Strategic Initiative, and Journal Archive funds

Add back for capitalization of in-house software development labor

Depreciation of in-house software development labor

Postretirement benefit-related changes other than net periodic cost

Change in unrestricted net assets

Changes in temporarily restricted net assets:

Contributions

Investment returns

Net assets released from restrictions

Reclassifications for donor redesignations

Change in temporarily restricted net assets

Change in permanently restricted net assets:

Contributions

Reclassifications for donor redesignations

Change in permanently restricted net assets

\section{Change in net assets}

Net assets, beginning of year

Net assets, end of year

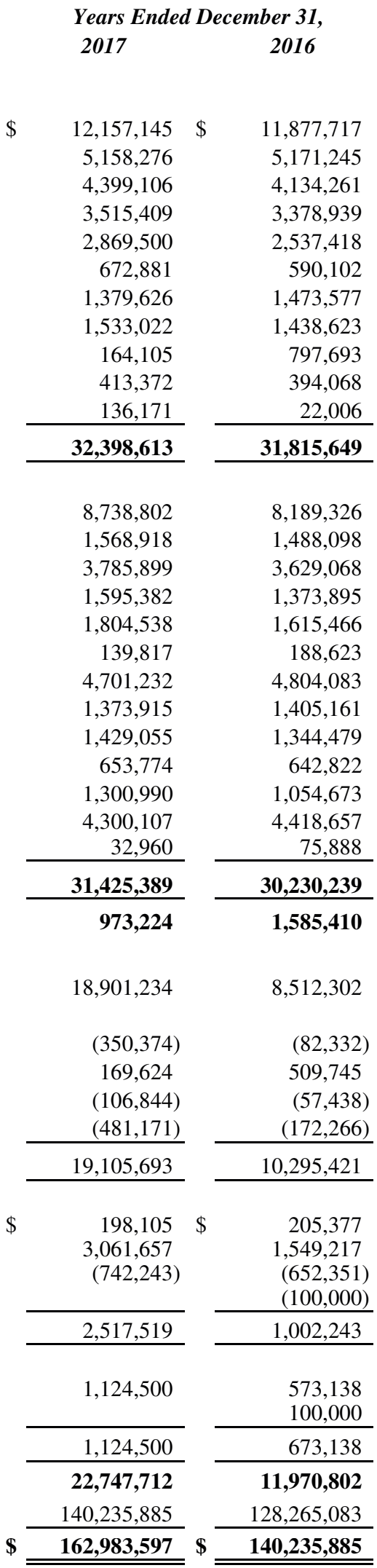


American Mathematical Society-Statements of Invested Funds As of December 31, 2017 and 2016

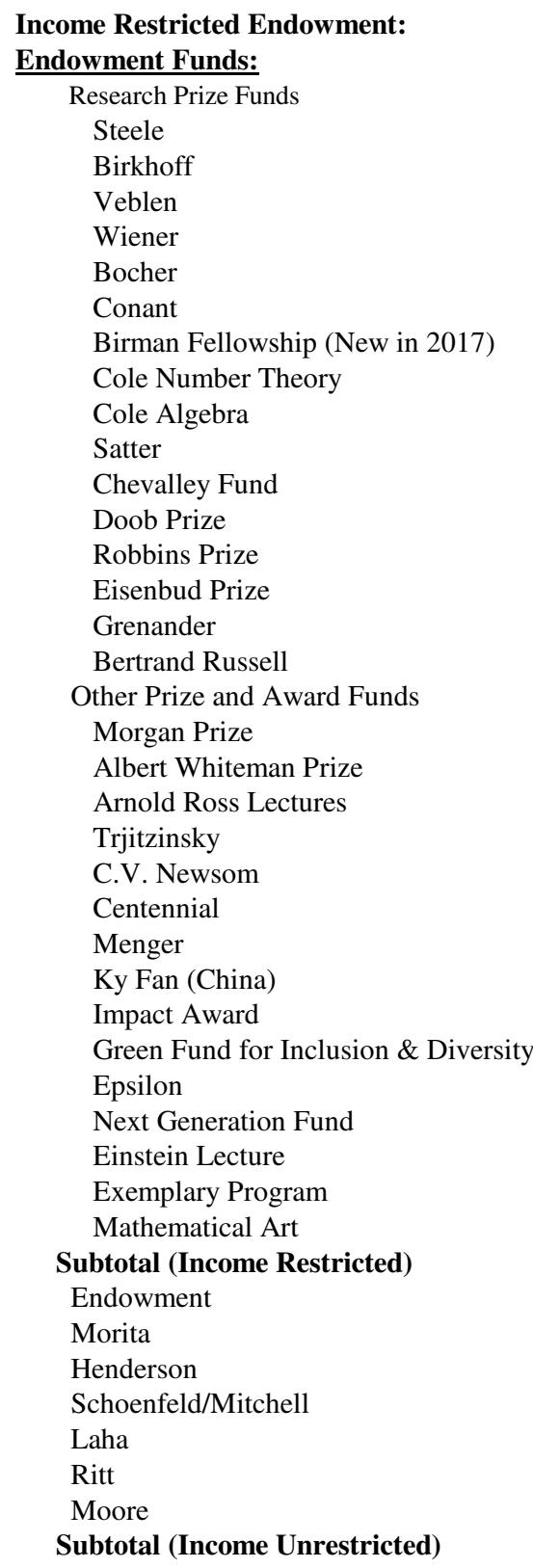

Total Endowment Funds

\section{Original Gift $\underline{\text { at } 12 / 31 / 17}$}

145,511

50,232

58,599

29,773

32,557

9,477

407,678

52,563

52,213

49,720

115,100

80,000

41,250

40,000

84,295

100,100

25,000

95,513

107,729

196,030

100,000

61,183

98,250

366,757

22,110

26,000

$2,271,243$

431,029

100,000

100,000

$\underline{20,000}$

$\mathbf{5 , 3 6 9 , 9 1 2}$

115,234

100,000

548,223

573,447

189,309

51,347

$\underline{2,575}$

$\mathbf{1 , 5 8 0 , 1 3 5}$

$\underline{6,950,047}$

Quasi-Endowment Funds (Board-Designated):

Journal Archive Fund

Young Scholars

Economic Stabilization Fund (ESF)

Backfile Digitization Fund

Strategic Initiative Fund

Kathleen Baxter Memorial Fund

Operations Support Fund (OSF)

Total Quasi-Endowment Funds

Owed to Operations

Beal Prize (Temporarily Restricted)

Total Invested Funds

12/31/17 Total Value

$\begin{array}{rr}927,598 & 810,858 \\ 116,556 & 101,799 \\ 103,060 & 90,090 \\ 61,757 & 53,985 \\ 62,738 & 54,843 \\ 61,820 & 54,040 \\ 470,204 & \\ 87,905 & 76,607 \\ 87,513 & 76,265 \\ 101,718 & 88,917 \\ 141,405 & 123,515 \\ 119,593 & 104,542 \\ 71,122 & 62,172 \\ 66,608 & 58,226 \\ 93,122 & 51,093 \\ 114,506 & 100,000\end{array}$

67,275

163,063

164,688

745,299

346,822

184,998

169,513

595,749

34,617

30,111

$3,626,530$

506,412

172,465

171,411

$\underline{34,282}$

$\mathbf{9 , 7 0 0 , 4 6 0}$

952,402

168,386

$5,021,196$

951,996

319,835

299,155

$\underline{28,199}$

$\mathbf{7 , 7 4 1 , 1 6 9}$

$\underline{17,441,629}$

$2,104,967$

$1,027,719$

$25,122,645$

80,702

345,822

320,396

$\underline{111,748,274}$

$140,750,525$

$3,248,803$

$\underline{1,558,779}$

$\$ \mathbf{\$ 7 , 9 5 0 , 0 4 7}$
12/31/16

Total Value

810,858

90,090

53,985

4,843

76,607

76,265

88,917

104,542

62,172

51,093

100,000

58,809

142,488

142,258

651,502

303,174

161,716

147,297

520,773

30,260

25,447

$3,074,571$

417,874

150,760

149,838

$\underline{29,968}$

7,913,687

$1,007,499$

178,541

$5,324,001$

$1,009,407$

339,123

317,196

$\underline{29,900}$

$8,205,667$

$\underline{16,119,354}$

$1,872,852$

896,980

$24,163,443$

68,401

310,657

281,288

94,606,082

122,199,703

449,077

$\underline{1,348,288}$

$\underline{\$ 140,116,422}$ 


\section{AMS SHORT COURSE}

\section{Sum of Squares:}

Theory and

Applications

\section{January 14-15, 2019, Baltimore, MD}

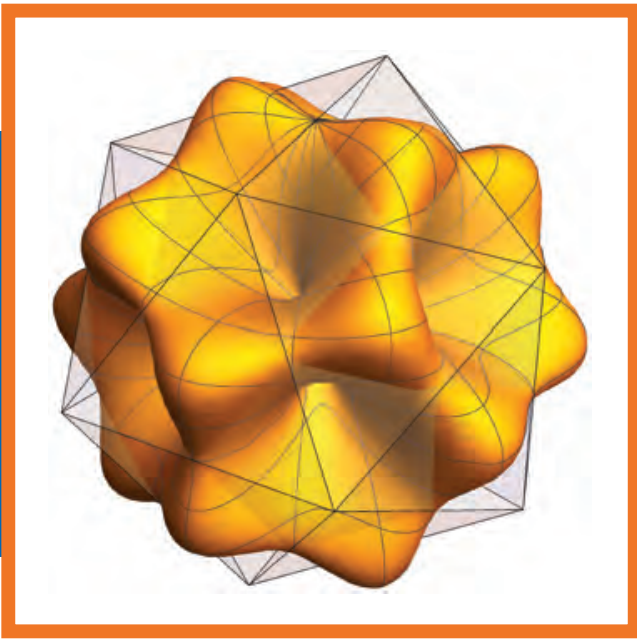

(in conjunction with the Joint Mathematics Meetings)

The American Mathematical Society's Short Courses connect mathematicians and students to emergent areas of applied mathematics through a series of survey lectures and activities. Short Courses are designed to introduce state-of-the-art research to a non-specialist audience, fueling their curiosity, discovery, and research.

In 2019, the Short Course lecturers focus on the theory and application of sums of squares (SOS) polynomials. These applications span a wide spectrum of mathematical disciplines from real algebraic geometry to convex geometry, combinatorics, real analysis, theoretical computer science, quantum information and engineering.

\section{Course Organizers:}

Pablo A. Parrilo, Massachusetts Institute of Technology

Rekha R. Thomas, University of Washington

\section{Lecture Topics:}

Overview of SOS polynomials,

Greg Blekherman, Georgia Institute of Technology

Lifts of Convex Sets,

Hamza Fawzi, University of Cambridge

\section{Engineering Applications,}

Georgina Hall, Princeton University
Theoretical Computer Science,

Ankur Moitra, Massachusetts Institute of Technology

Algebraic Geometry,

Mauricio Velasco, Los Andes University

Geometry of Spectrahedra,

Cynthia Vinzant, North Carolina State University

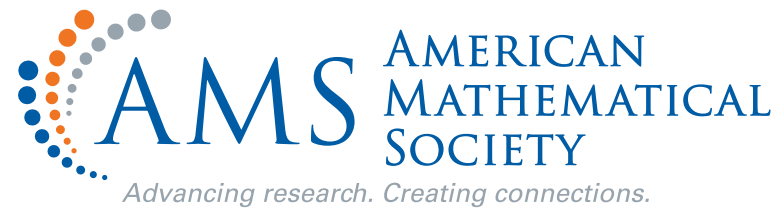




\section{AMS Employment Center}

\section{Baltimore Convention Center, Baltimore, Maryland}

January 16-18,2019

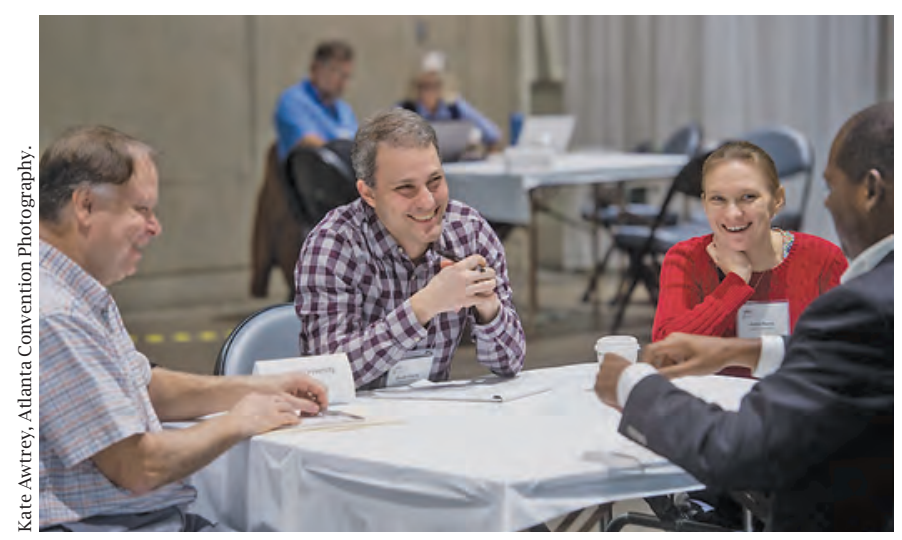

The Employment Center offers a convenient, safe and practical meeting place for employers and job seekers attending the Joint Mathematics Meetings (JMM). The focus of the Employment Center is on PhD-level mathematical scientists and those that seek to hire them from academia, business, and government.

\section{Employment Center Schedule:}

December 27, 2018, is the deadline for table registration. After this date, only "One Day Tables" will be available for purchase. This is also the deadline to register for the JMM badge, needed for admittance to the EC, at Advance Registration Prices.

Hours of Operation (Please note there is no access to the EC prior to the opening times listed):

Wednesday, January 16, 2019 - 8:00 am-5:30 pm

Thursday, January 17, 2019 - 8:00 am-5:30 pm

Friday, January 18, 2019 - 8:00 am-5:30 pm

Location: Exhibit Hall E, Baltimore Convention Center, Baltimore, Maryland.

Do not schedule an interview to begin until 15 minutes after opening.

\section{Employment Center Web Services}

Employment Center registration information should be accessed through the MathJobs.Org system. The website and all information will be available beginning in early September 2018 and will remain accessible through January 18, 2019, (the last day of the Employment Center). While some schools may delay appointment setting until late December 2018, virtually all scheduling will be done before any Joint Mathematics Meetings (JMM) travel takes place, so applicants should expect few or no additional appointments to be available after arrival. Registering onsite, for applicants, serves no real purpose.

\section{No Admittance Without a JMM Badge}

All applicants and employers planning to enter the Employment Center-even just for one interview-must present a 2019 JMM Registration badge. Meeting badges are obtained by registering for the JMM and paying a meeting registration fee. The advanced registration deadline is December 27, 2018. See the JMM website at: jointmathematicsmeetings.org/jmm for registration instructions and rates.

\section{Employers: Choose a Table}

There are three table types available for employers, based on the number of interviewers who will be present at any given time:

- One or two interviewers per table in the "Quiet Area” (US \$355), each additional table (US\$200).

- Three to six interviewers per table in the "Committee Table” area (US \$445), each additional table (US\$220).

- "One Day Tables" allow for on-site interviewing for one day without placing an ad. These tables, which can accommodate up to three interviewers, may be purchased through January 18, 2019. The fee is (US\$195). Please register online at www.mathjobs.org and choose the "EC-One Day Table purchase."

"Free electricity is supplied to every table with purchase of the table.

All Employment Center data and registration must be entered on the MathJobs.Org website. An existing account 
can be used for accessing Employment Center services and for paying applicable fees. If no account exists, participants can start an account solely for Employment Center use.

Employers are expected to create their own interview schedules as far in advance as possible by using the assisted-email system in MathJobs.Org or by using other means of communication. Please do not schedule an interview to begin until fifteen minutes after the Employment Center opens.

Please mark appointments as confirmed in your MathJobs.Org account, as this will allow the appointments to display in the applicants' schedules. At the time of interview, meet the applicant in the on-site waiting area and escort him or her to your table.

\section{Employers: How to Register}

- Registration runs from early September 2018 through December 27, 2018, at the following website: www. mathjobs.org. After December 27, only "One-Day Tables" will be available. They should be reserved and paid for through MathJobs.Org.

- Use your existing Mathjobs.Org account or create a new Employer account at www. mathjobs . org. Once a table is reserved, the ad can be placed at any time (or never) and will run until late January.

- For new users of MathJobs.Org, click the NEW EMPLOYER link on the main page of www . mathjobs . org. Choose your table type and fill out the New Employer Form.

- For existing users of MathJobs.Org, go to www. mathjobs.org. Log into your existing account. Purchase a table by clicking the "EmpCent" logo in the menus along the top tool bar. Use the "buy tables" link. Then post a job using the NewJob link or attach an existing job to your table.

- Each person who will need to enter the Employment Center area must have a meeting badge (obtained by registering for the JMM and paying a meeting registration fee).

To display an ad on-site, and use no Employment Center services at all, submit your one-page paper ad on-site in Baltimore to the Employment Center staff. There is no fee for this service.

For complete information, visit www.ams.org/ emp-reg.

\section{Applicants: Making the Decision to Attend}

- Past attendees have pointed out that all interviews are arranged in advance, and there is no opportunity to make connections on-site if it has not happened before the meeting.

- The Employment Center offers no guarantees of interviews or jobs. Hiring decisions are not made during or immediately following interviews. In the current job market, the ratio of applicants to employers is about 6:1, and many applicants go completely unnoticed.
- There will ordinarily be no research-oriented postdoctoral positions listed or discussed at the Employment Center.

- Interviews will go to applicants who applied to jobs during the fall and are now being sought out by the institutions for in-person meetings during the JMM.

- There will be no opportunity to speak to employers without a prearranged interview, and no walk-up job information tables. Scheduling of interviews will be complete prior to the JMM.

The majority of Employment Center employers are academic departments of mathematical sciences seeking to meet a short list of applicants who applied for their open positions during the fall. Each year, a few government or industry employers are present. Often, they are seeking US citizens only due to existing contracts.

All job postings are available on the website in advance, and now that this electronic service is in place, there is no other messaging conducted on paper.

Please visit the Employment Center website for further advice, information and program updates at www. ams.org/emp-reg

\section{Applicants: How to Register}

- Early registration is vital since most employers will finalize schedules before arriving in Baltimore.

- To register, applicants should log into their MathJobs. Org accounts or create a new account, look for the EmpCent icon across the top tool bar and mark that they will be attending by clicking the link, "click here if you are attending the Employment Center." You can then upload documents and peruse the list of employers attending and the positions available. You do not have the option to request an interview with an employer. However, if you are interested in any position, you can apply to the job. The employer will be aware that you are also attending the event and will contact you directly if interested in setting up an interview.

There are no Employment Center fees for applicants; however, admission to the Employment Center room requires a 2019 JMM badge, obtainable by registering (and paying a fee) for the JMM. To register for the meeting, go to the website: jointmathematicsmeetings.org/jmm.

It is possible to attend one or more privately arranged interviews without an official Employment Center registration; however, a meeting badge is required to access the interview room.

Applicants should keep track of their interview schedules. If invited for an interview at a conflicting time, please ask the employer to offer a new time or suggest one.

For complete information, visit www.ams.org/ emp-reg.

Questions about the Employment Center registration and participation can be directed to Pamela Morin, AMS Professional Programs Department, at 800-321-4267, ext. 4060 or by e-mail to emp-info@ams.org. 


\section{AMS Centennial Research Fellowship Program}

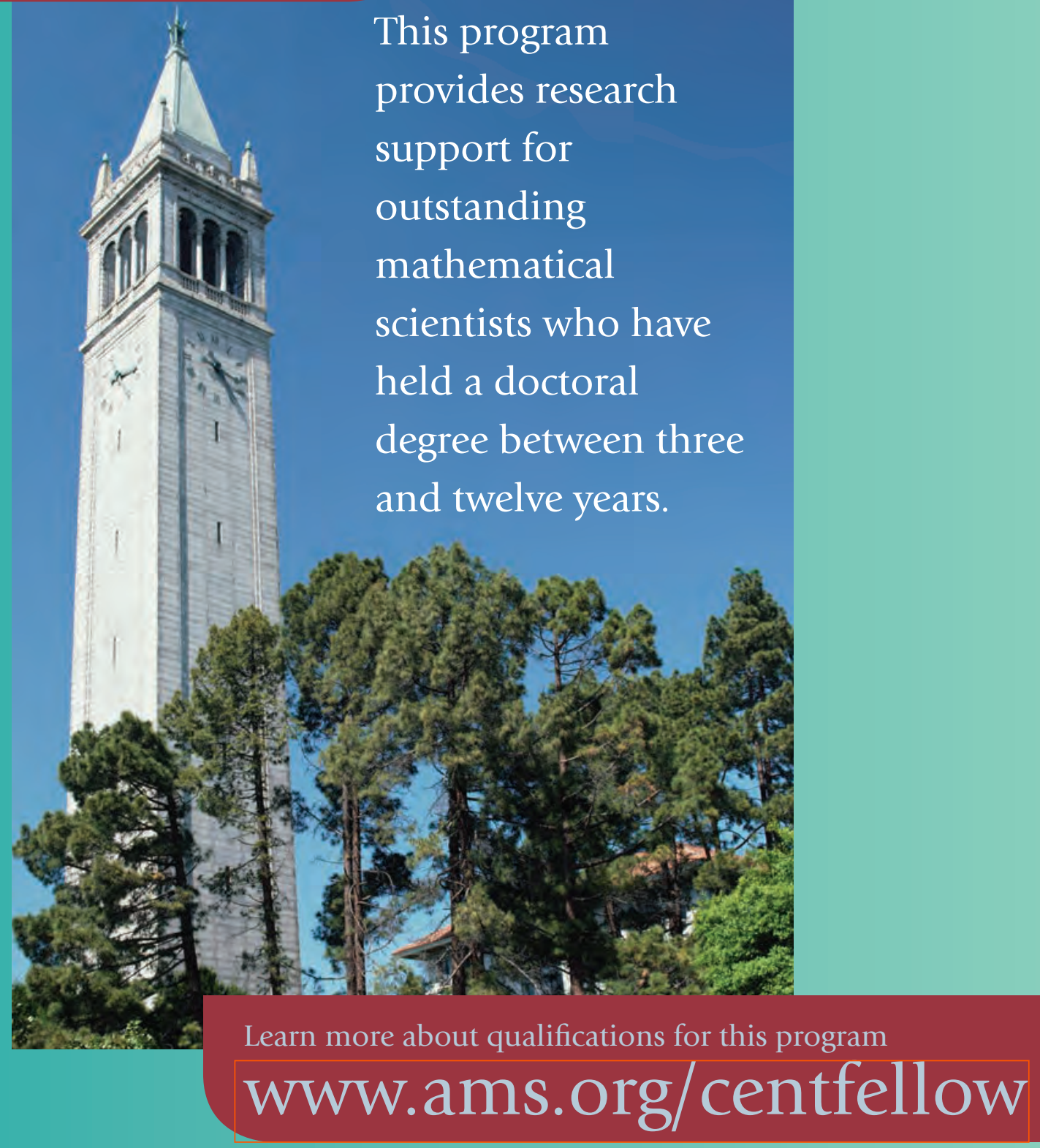

Application deadline: December 1, 2018

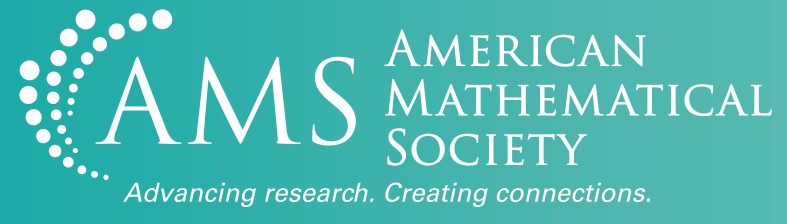




\section{Mathematics People}

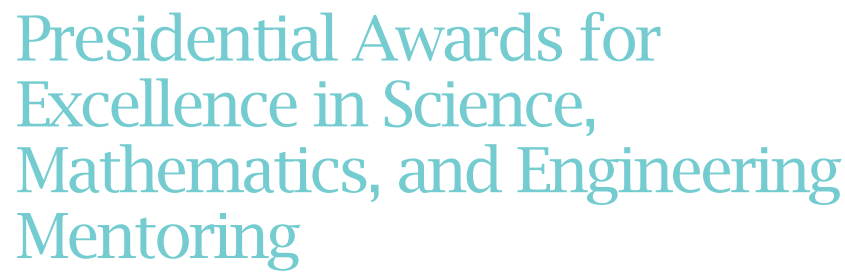

Two educators and an educational program whose work involves the mathematical sciences were selected to receive the Presidential Awards for Excellence in Science, Mathematics, and Engineering Mentoring.

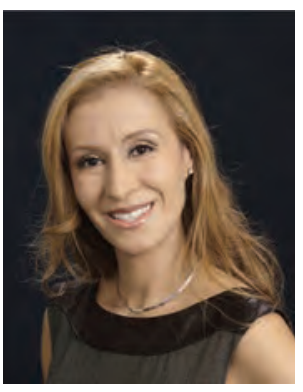

ERIKA CAMACHO of Arizona State University was honored for her mentoring philosophy, which centers on the core belief that every individual is capable of achievement and that each individual should be appropriately challenged according to her or his particular needs. Camacho has held Sloan and Ford Foundation Fellowships and has received a Leader and Mentor in Undergraduate Research Citation from the National Se-

curity Agency, the National Latina Leadership Award from the National Hispanic Women's Corporation, the SACNAS Distinguished Undergraduate Institution Mentor Award, the Outstanding Mentor Award from the Department of Mathematics of the University of Texas at Arlington, and the Outstanding Latino/a Faculty in Higher Education: Research/Teaching Award from the American Association of Hispanics in Higher Education. She is a member of the AMS Council, Committee on Education, and Young Scholars Award Committee.

GLENN S. LEE of Waialua High and Intermediate School, Waialua, Hawaii, has taught mathematics and science to youngsters from the rural, semi-isolated community of Waialua, where social services, workforce training opportunities, and alternative agricultural programs have been limited. His activities have enabled students from groups traditionally underrepresented in science, technology, engineering, and mathematics-Pacific Islanders, African Americans, and native Hawaiians-to successfully complete a high school program, pursue postsecondary education, and acquire the knowledge and skills needed to compete in a twenty-first-century workforce environment.

The ENHANCING DIVERSITY IN GRADUATE EDUCATION (EDGE) program was honored for providing introductions

to key figures in the scholarly field, gaining visibility for one's research and teaching accomplishments, and making key connections with public and private organizations for career professional work. Participants in the program constituted thirty-five percent of PhDs granted to African American women in the mathematical sciences during the years 2005 to 2009. The EDGE program was the recipient of the AMS Mathematics Programs That Make a Difference Award in 2007.

-From a National Science Foundation announcement

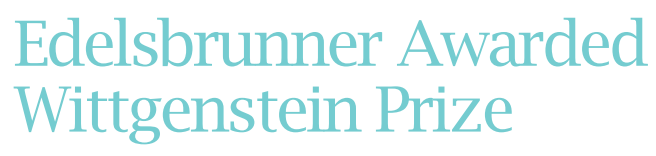

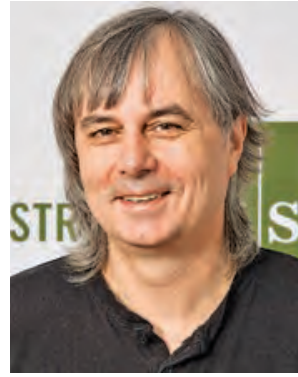

Herbert Edelsbrunner
HERBERT EDELSBRUNNER of the Institute of Science and Technology Austria (IST Austria) has been awarded the Wittgenstein Prize of the Austrian Science Fund.

Edelsbrunner is "one of the world's leading researchers in computational geometry and topology [who] has played a significant role in laying the foundations of his field." According to the prize citation, Edelsbrunner "is pursuing three main research areas, each with the goal of further developing computational topology and thus opening up new areas of application. These emphases include stochastic, algebraic, and geometric questions within the field of topological data analysis. All three aspects are necessary to gain the-possibly revolutionary-insights necessary to understand the microscopic world of materials."

Edelsbrunner received his $\mathrm{PhD}$ in technical mathematics from Graz University of Technology in 1982. In 1985 he joined the faculty of the University of Illinois at UrbanaChampaign. In 1996, he cofounded the software company Geomagic. In 1999 he moved to Duke University, and he joined IST Austria in 2009. He was awarded the Alan T. Waterman Award of the National Science Foundation in 1991. He was elected to the American Academy of Arts and Sciences in 2005, to the German Academy of Sciences Leopoldina in 2008, and to the inaugural class of Fellows of the European Association for Theoretical Computer 
Science in 2014. He is a member of the Austrian Academy of Sciences and of Academia Europaea.

The prize carries a cash award of 1.5 million euros (approximately US $\$ 1,750,000$ ) to support the mathematician's research for a period of five years.

\section{-From an IST Austria announcement}

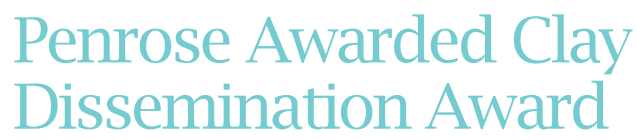

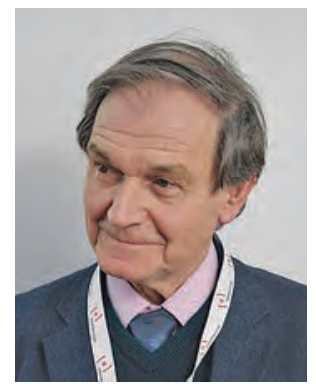

Sir Roger Penrose
SIR Roger Penrose of the University of Oxford has been selected to receive the Clay Award for Dissemination of Mathematical Knowledge by the Clay Mathematics Institute "in recognition of his outstanding contributions to geometry, relativity, and other branches of mathematics, and of his tireless work in explaining mathematical ideas to the public through popular books, public lectures, and broadcasts."

The citation goes on: "Roger Penrose has made extraordinary and wide-ranging contributions to mathematics and its applications, often making novel and inspiring connections across disciplinary boundaries. He started out in algebraic geometry under W. V. D. Hodge and J. A. Todd at Cambridge, but within a few years of completing his $\mathrm{PhD}$ thesis on Tensor Methods in Algebraic Geometry, he had laid the foundations of the modern theory of black holes with his celebrated paper on gravitational collapse. For this work he was awarded the Wolf Prize in Physics, with Stephen Hawking. His exploration of foundational questions in relativistic quantum field theory and quantum gravity, based on his twistor theory, had a huge impact on differential geometry. In other areas, a paper that he wrote as a student introduced what is now known as the Moore-Penrose inverse. His discovery of 'Penrose tilings' spawned a new field in geometry, as well as having an impact in crystallography. It was Penrose who first suggested that Church's lambda calculus could be a powerful tool in exploring programming language semantics. In his collaboration with M. C. Escher, he crossed over into the visual arts.

"His work in public engagement goes beyond the simple exposition of mathematics. In his popular books, he has set out, with considerable success, to engage the public with the development of his ideas and to draw them into an interconnected world of intellectual endeavour which is the common property of all, not just of 'specialists.' So his books on artificial intelligence aim not just to inform his readers about deep mathematical ideas of relevance to the subject but also to enable and encourage participation of the public in discussion of the nature of consciousness.

"He is an energetic public lecturer, traveling widely and attracting large audiences because he speaks in direct and engaging terms about mathematical ideas that he thinks are worth communicating and that his audiences think are worth trying to understand. Many of his lectures have been recorded and made available online, along with recordings of television appearances. They have been viewed by hundreds of thousands of people."

The prize is awarded in recognition of personal contributions to research in mathematics at the highest level, as well as of distinction in explaining recent advances to the public at large.

\section{-From a CMI announcement}

\section{Kashiwara Awarded Kyoto Prize}

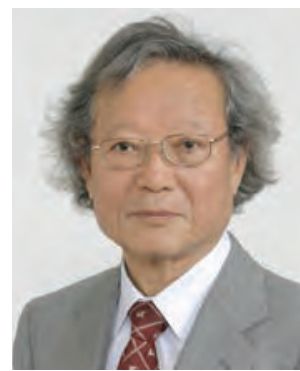

Masaki Kashiwara
MASAKI KASHIWARA of Kyoto University has been awarded the Kyoto Prize in Basic Sciences for Outstanding Contributions to a Broad Spectrum of Modern Mathematics, including his work in advancing $D$ module theory.

According to the prize citation, Kashiwara "established the theory of $D$-modules, thereby playing a decisive role in the creation and development of algebraic analysis. His numerous achievements-including the establishment of the Riemann-Hilbert correspondence, its application to representation theory, and construction of crystal basis theory-have exerted great influence on various fields of mathematics and contributed strongly to their development." The prize is awarded by the Inamori Foundation to individuals who have contributed significantly to the scientific, cultural, and spiritual betterment of humankind. The prize carries a cash award of 100 million yen (approximately US\$900,000).

\section{-From an Inamori Foundation announcement}

\section{Simons Foundation Investigators Named}

The Simons Foundation has named the Simons Foundation Investigators for 2018. Following are the investigators whose work involves the mathematical sciences.

\section{Mathematics:}

ANDRÉ ARROJA NEVES of the University of Chicago is a leading figure in geometric analysis with important contributions ranging from the Yamabe problem to geometric flows. Jointly with Fernando Marques, he transformed the field by introducing new ideas and techniques that 
led to the solution of several open problems which were previously out of reach. Together or with coauthors, they solved the Willmore conjecture, the Freedman-He-Wang conjecture in knot theory, and Yau's conjecture on the existence of minimal surfaces in the generic case.

SYLVIA SERFATY of New York University works on understanding the behavior of physical systems via the tools of mathematical analysis, partial differential equations, and probability. A large part of her work has focused on the Ginzburg-Landau model of superconductivity, tackling and largely solving the problem of why and when vortices form the so-called Abrikosov triangular lattices. She has recently turned her attention to the statistical mechanics and dynamics of Coulomb-type systems and other manyparticle systems with long-range interactions.

AKSHAY VENKATESH of Stanford University works in number theory and related fields. A major focus of his recent work has been the topology of locally symmetric spaces, in particular on new phenomena that occur when one leaves the setting of Shimura varieties. In that context, he has postulated and gathered evidence for a deep relationship between this topology and the motivic cohomology of certain algebraic varieties. In August, Venkatesh became a 2018 Fields medalist.

\section{Theoretical Computer Science:}

Constantinos DASKALAKIs of the Massachusetts Institute of Technology works on computation theory and its interface with game theory, economics, probability theory, statistics, and machine learning. His work has resolved long-standing open problems about the computational complexity of the Nash equilibrium, the mathematical structure and computational complexity of multi-item auctions, and the behavior of machine-learning methods such as the expectation-maximization algorithm. He has obtained computationally and statistically efficient methods for statistical hypothesis testing and learning in high-dimensional settings, as well as results characterizing the structure and concentration properties of highdimensional distributions.

RAN RAZ, Princeton University. Raz's main research area is computational complexity theory, with a focus on proving lower bounds for computational models. He works on Boolean and algebraic circuit complexity, communication complexity, probabilistically checkable proofs, and interactive proof systems. In the last years, he studied relations between communication complexity and information complexity of communication protocols and worked on unconditional lower bounds on the number of samples needed for learning, under memory constraints.

\section{Mathematical Modeling of Living Systems:}

Claudia Clopath, Imperial College of Science, Technology, and Medicine. Claudia Clopath's work has contributed to our understanding of learning in the brain. She has developed mathematical models of learning to link the plasticity observed experimentally at the neural level to the function at the network and behavioral levels.

LUCY COLWELL of the University of Cambridge has demonstrated that the three-dimensional structure of proteins can be determined from large sequence alignments. Her current research develops methods for relating phenotype to genotype, using large data sets from high throughput biological experiments, focusing mainly on proteins, small molecules and nucleic acids.

ELENI KATIFORI of the University of Pennsylvania is a recipient of a Burroughs Wellcome Career Award at the Scientific Interface and a National Science Foundation Career Award. Her research interests are primarily theoretical and span broad areas from soft matter physics with a focus on biologically inspired physics to thin shell elasticity and biological transport networks.

DANIELA WITTEN of the University of Washington is a leader in the field of statistical machine learning. Her work focuses on the development of supervised and unsupervised learning methods for making sense of large and messy data sets arising from genomics, neuroscience, and other fields. She has developed new frameworks for performing clustering, graphical modeling, and matrix decompositions in the high-dimensional setting.

The Simons Investigators program provides a stable base of support for outstanding scientists, enabling them to undertake long-term study of fundamental questions.

-From a Simons Foundation announcement

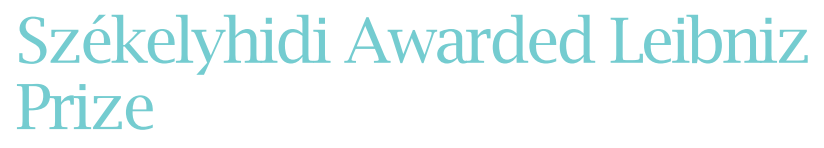

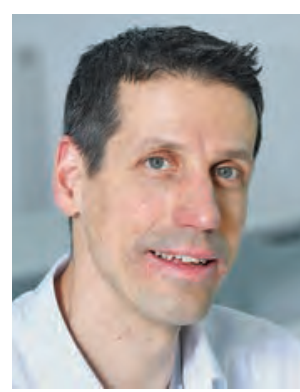

László Székelyhidi
LÁSZLÓ SZÉKELYHIDI of the University of Leipzig was awarded the 2018 Leibniz Prize for his research in the theory of partial differential equations. According to the prize citation, "the methods he developed have enriched the interchange between geometry and analysis in mathematics. His new insights have a significance far beyond his own research area, for example in the understanding of Euler equations in hydrodynamics and elasticity theory in continuum mechanics. Euler equations have posed a major challenge in mathematics for over two hundred years. Together with Camillo De Lellis, Székelyhidi has developed new approaches to the construction of non-smooth solutions to Euler equations. These led to a complete proof of Onsager's conjecture, which states that solutions below a certain Hölder continuity do not conserve energy but can reduce or, in a non-physical way, increase it. In the field of elasticity theory in continuum mechanics, Székelyhidi, in his doctoral thesis, succeeded in constructing a poly- 
convex variation problem with an extremal that cannot be differentiated at any point. In collaboration with Daniel Faraco, he achieved another scientific breakthrough with a compactness result, which is closely related to Morrey's conjecture."

Székelyhidi received his PhD in 2003 from the University of Leipzig. He held research positions at Princeton University and ETH Zurich. In 2007 he was appointed professor at the University of Bonn, and he has been at the University of Leipzig since 2011. In 2010 he received the Oberwolfach-Preis für Analysis und Angewandte Mathematik. He was an invited speaker at the ICM in Seoul in 2014 and a plenary speaker at ICMP Chile in 2015.

\section{_From a German Research Foundation announcement}

\section{Kwaśnicki Awarded Gordin Prize}

MATEUSZ KWAŚNICKI of Wrocław University of Science and Technology has received the 2018 European Mathematical Society Gordin Prize for his outstanding contributions to the spectral analysis of Lévy processes. The prize honors the memory of Mikhail Gordin and is awarded to a junior mathematician from an Eastern European country working in probability or dynamical systems. The award consists of a cash prize of US\$4,000.

\section{-From an EMS announcement}

\section{European Girls' Mathematical Olympiad}

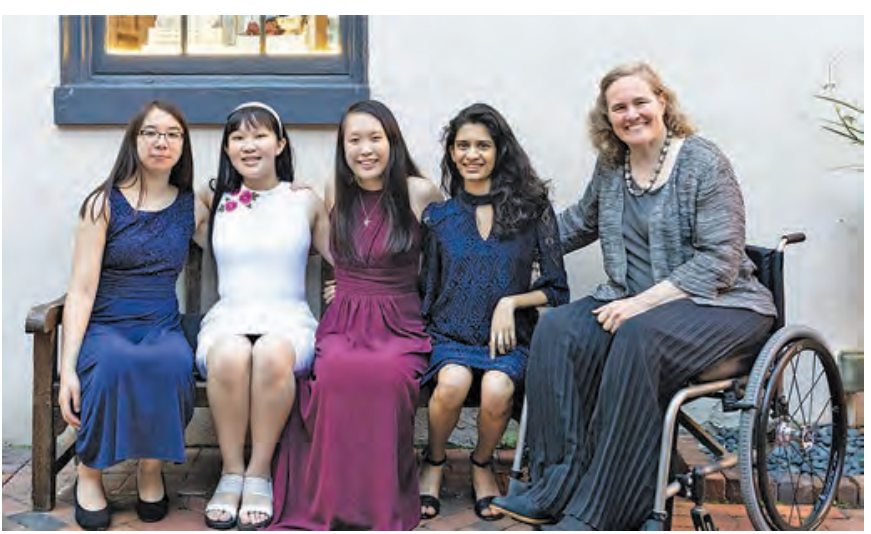

L-R: Wanlin Li, Catherine Wu, Emily Wen, Megan Joshi, and Deanna Haunsperger.

The team from the Russian Federation took first place at the European Girls' Mathematical Olympiad (EGMO) held in Florence, Italy, in April 2018. The team from the United States took second place, and the team from the United Kingdom finished third. The 2018 EGMO US team members were CATHERINE WU, WANLIN LI, EMILY WEN, and
MEGAN JosHI. Wu and Li received gold medals; Joshi received a silver medal; and Wen earned a bronze medal. Students from fifty-two countries participated in the competition for female high school students. Seventeen students were awarded gold medals, thirty-nine received silver, and fifty-two won bronze. The US team is organized by the Mathematical Association of America as part of the MAA American Mathematics Competitions.

-From an MAA announcement
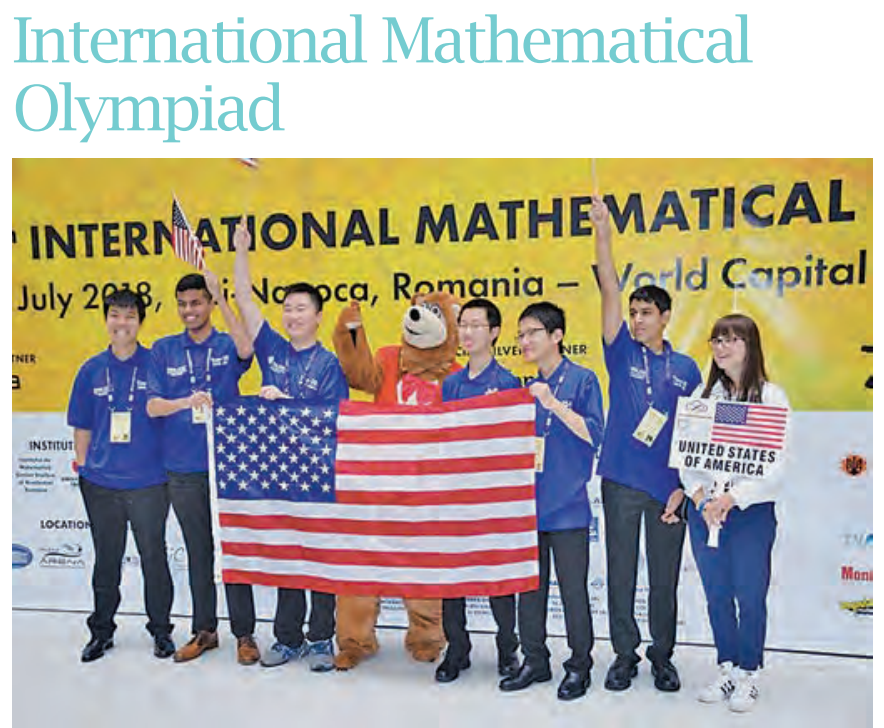

L-R: Michael Ren, Adam Ardeishar, James Lin, Vincent Huang, Andrew Gu, and Mihir Singal.

The 2018 International Mathematical Olympiad was held in Cluj-Napoca, Romania, from July 3 to 14. The team from the United States took first place. The team from Russia finished second, and the team from China was awarded third place. The US team consisted of ADAM ARDEISHAR, ANDREW Gu, VinCENT HuANG, JAMES LiN, MiCHAEL REN, and MiHIR SINGHAL. Gu, Huang, and Lin were members of the 2017 team. Lin earned a perfect score. Gu, Huang, Lin, Ren, and Singhal won gold medals; Ardeishar earned a silver medal. The team was coached by Po-Shen Loh of Carnegie Mellon University. The sixtieth competition will be held in the United Kingdom in 2019.

-From an MAA announcement 


\section{SIAM Prizes}

The Society for Industrial and Applied Mathematics (SIAM) has awarded several prizes for 2018.

The Martin Kruskal Lectureship was awarded to

MiCHAEL I. WEINSTEIN of Columbia University. The prize is given for significant contributions to the mathematical theory of nonlinear waves and coherent structures.

The SIAM-MOS George B. Dantzig Prize was awarded to ANDRZEJ RUSZCZYNSKI of Rutgers University and ALEXANDER SHAPIRO of the Georgia Institute of Technology. The prize is awarded for original research that, by its originality, breadth, and depth, is having a major impact on the field of mathematical optimization.

The Gábor Szegő Prize was awarded to THOMAS TROGDON of the University of California Irvine. The SIAM Activity Group on Orthogonal Polynomials and Special Functions awards the prize every two years to one individual in his or her early career for outstanding research contributions in the area of orthogonal polynomials and special functions.

The AMS-MAA-SIAM Gerald and Judith Porter Public Lecture was awarded to MOON DUCHIN of Tufts University. Her lecture was titled "Political Geometry: Voting Districts, 'Compactness,' and Ideas about Fairness.” This lecture on a mathematical topic accessible to the broader community is given each year at the Joint Mathematics Meetings.

The 2018 SIAM-MOS Lagrange Prize in Continuous Optimization was awarded to FRANCIS BACH of the INRIASIERRA Project, NiCOLAS LE Roux of Google Brain, and MARK SCHMIDT of the University of British Columbia. The prize is awarded every three years for an outstanding contribution in the area of continuous optimization published in the six calendar years prior to the award year.

\section{-From SIAM announcements}

\section{Prizes of the London Mathematical Society}

The London Mathematical Society has awarded a number of prizes for 2018 .

The Pólya Prize was awarded to KAREN VogTMANN of Warwick University for her profound and pioneering work in geometric group theory, particularly the study of automorphism groups of free groups.

The Fröhlich Prize was awarded to Francesco MEzZADRI of the University of Bristol for his profound and wide-ranging contributions to random matrix theory and its applications.

A Senior Berwick Prize was awarded to MARC LEVINE of the University of Duisburg-Essen in recognition of his paper "A Comparison of Motivic and Classical Stable Homotopy Theories," Journal of Topology 7 (2014), no. 2.

The Hirst Prize has been awarded to JEREMY GRAY of the Open University and the University of Warwick for his research and books on the history of mathematics, espe- cially differential equations and geometry in and around the nineteenth century.

The Anne Bennett Prize was awarded to LotTe HoLLANDS of Heriot-Watt University in recognition of her outstanding research at the interface between quantum theory and geometry and of her leadership in mathematical outreach activities.

Whitehead Prizes were awarded to the following individuals:

CAUCHER BIRKAR of Cambridge University in recognition of his outstanding research in higher dimensional algebraic geometry, most prominently his recent groundbreaking finiteness results on Fano varieties and Mori fiber spaces. In August, Birkhar became a 2018 Fields medalist.

ANA CARAIANI of Imperial College London for her important contributions to the Langlands program.

HEATHER HARRINGTON of the University of Oxford for her outstanding contributions to mathematical biology, which have generated new biological insights using novel applications of topological and algebraic techniques.

VALERIO LUCARINI of the University of Reading for his work applying the ideas and methods of statistical physics to the theory and modeling of climate dynamics and for his leadership in the field of mathematics applied to climate science.

FILIP RINDLER of the University of Warwick for his solutions to fundamental problems on the border between the theory of partial differential equations, calculus of variations, and geometric measure theory.

PÉTER VARJú of the University of Cambridge for his deep and groundbreaking contributions to analysis and probability on algebraic structures.

-From an LMS announcement

\section{Photo Credits}

Photo of Erika Camacho courtesy of AHHE.

Photo of Herbert Edelsbrunner courtesy of IST Austria.

Photo of Sir Roger Penrose courtesy of Cirone-Musi, Festival della Scienza.

Photo of Masaki Kashiwara courtesy of the Inamori Foundation.-oPhoto of László Székelyhidi courtesy of DFG.

Photos of the EGMO and IMO winners courtesy of MAA. 


\section{SUPPORT JMM CHILD GARE GRANTS}

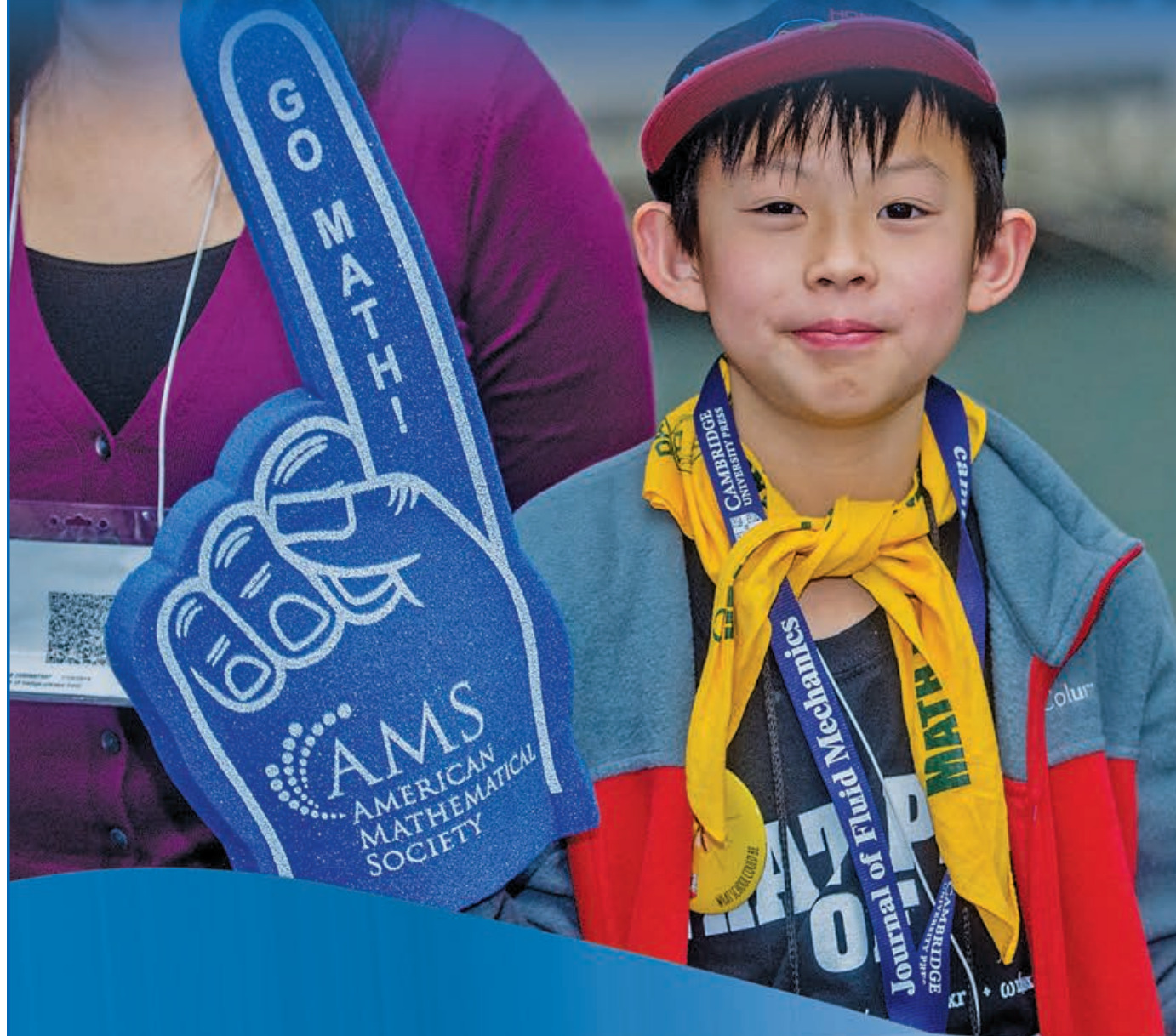

JMM Child Care Grants help early career scholars attend the Joint Mathematics Meetings at a critical time in their professional lives. These modest grants give mathematicians flexibility in arranging care for their children.

To learn more or give online: wWw.ams.org/child-care-grants 


\section{Mathematics Opportunities}

\section{Listings for upcoming math opportunities to appear in Notices may be submitted to notices@ams.org .}

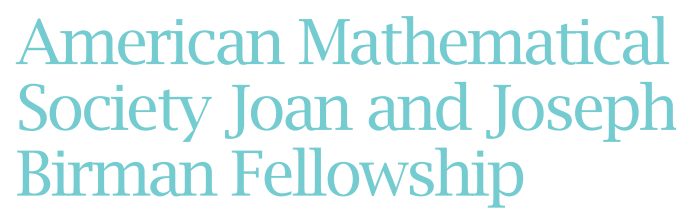

Invitation for Applications for Awards for 2019-2020 Deadline December 1, 2018

Description: The Joan and Joseph Birman Fellowship for Women Scholars is a mid-career research fellowship specially designed to fit the unique needs of women. The fellowship is open only to women and was established in 2017, made possible by a generous gift from Joan and Joseph Birman. One fellowship in the amount of US\$50,000 will be awarded for the 2019-2020 academic year.

Eligibility: The fellowship seeks to address the paucity of women at the highest levels of research in mathematics by giving exceptionally talented women extra research support during their mid-career years. The most likely awardee is a mid-career woman, based at a U.S. academic institution, with a well-established research record in a core area of mathematics. The fellowship will be directed toward those for whom the award will make a real difference in the development of their research careers. Candidates must have a carefully thought-through research plan for the fellowship period. Special circumstances (such as time taken off for care of children or other family members) may be taken into consideration in making the award. The fellowship can be used to provide additional time for research of the awardee or opportunities to work with collaborators. This may include, but is not limited to, course buy-outs, travel money, child care support, or support to attend special research programs.

Deadline: Applications will be accepted starting September 1, 2018. The deadline for receipt of applications is December 1, 2018. The award recipient will be announced in February 2019.

Application information: Find Birman application information at www.ams.org/programs/ams-fel1owships /Birman-fe11ow. For questions, contact the Professional Programs Department, American Mathematical Society, 201 Charles Street, Providence, RI 02904-2294; prof-serv@ams.org; 401-455-4096.

\section{*NSF Graduate Research Fellowships}

The National Science Foundation's Graduate Research Fellowship Program recognizes and supports outstanding graduate students in NSF-supported science, technology, engineering, and mathematics disciplines who are pursuing research-based master's and doctoral degrees at US institutions. Fellows receive a three-year annual stipend, opportunities for international research and professional development, and the freedom to conduct their research at any accredited US institution of graduate education. The deadline for the mathematical sciences is October 26, 2018. For further information, visit www.nsf.gov /funding/pgm_summ.jsp?pims_id=6201.

-From NSF announcements

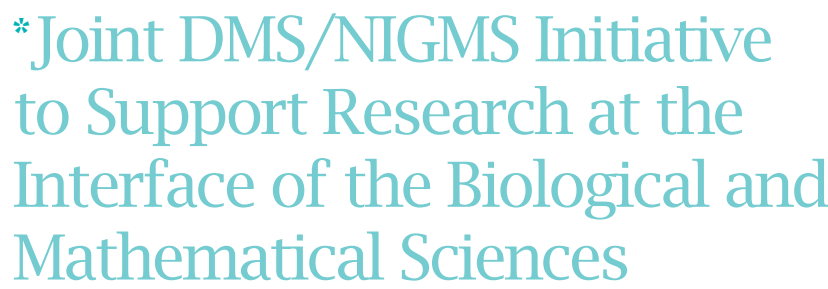

The Division of Mathematical Sciences at the National Science Foundation and the National Institute of General Medical Sciences at the National Institutes of Health support fundamental research in mathematics and statistics necessary to answer questions in the biological and biomedical sciences. This program is designed to encourage new collaborations, as well as to support innovative activities by existing teams. The submission window for full proposals is October 1-18, 2018. See www.nsf.gov /funding/pgm_summ.jsp?pims_id=5300. 


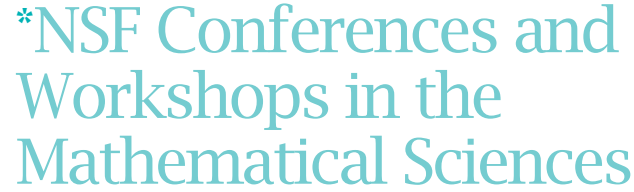

The National Science Foundation (NSF) supports conferences, workshops, and related events (including seasonal schools and international travel by groups). Proposals for such activities may request funding of any amount and for durations of up to three years. Proposals may be submitted only by universities and colleges or by nonprofit nonacademic institutions and must be submitted to the appropriate disciplinary program in accordance with the lead-time requirements specified on the program web page. For more information, see www.nsf.gov/funding /pgm_summ.jsp?pims_id=11701\&org=DMS

-NSF announcement

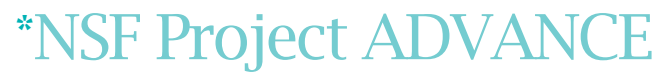

The ADVANCE program of the National Science Foundation focuses on funding projects that support systemic change for gender equity in STEM academic careers. The deadline for letters of intent is December 12, 2018. See www.nsf.gov/funding/pgm_summ.jsp?pims _id=5383\&org=DMS\&se1_org=DMS\&from=fund.

-NSF announcement

\section{Mathematics Research Communities 2019}

The AMS invites mathematicians just beginning their research careers-those who are close to finishing their doctorates or have recently finished-to become part of Mathematics Research Communities (MRC), a unique and successful program that builds social and collaborative networks through which individuals inspire and sustain each other in their work. Women and underrepresented minorities are especially encouraged to participate. Partially supported by the National Science Foundation, the structured program engages and guides all participants as they start their careers. Those accepted into the program will receive support for the summer conference and will be partially supported for their participation in the Joint Mathematics Meetings that follow in January 2020. The program also includes discussion networks by research topic and a longitudinal study of early-career mathematicians.

\footnotetext{
"The most up-to-date listing of NSF funding opportunities from the Division of Mathematical Sciences can be found online at: www.nsf.gov/dms and for the Directorate of Education and Human Resources at www.nsf.gov/dir/index.jsp?org=ehr. To receive periodic updates, subscribe to the DMSNEWS listserv by following the directions at www.nsf.gov/mps/dms/about.jsp.
}

Three conferences will be held in summer 2019 on the following topics:

Week 1: June 2-8, 2019: Geometric Representation Theory and Equivariant Elliptic Cohomology Organizers:

- Dan Berwick-Evans (University of Illinois at UrbanaChampaign)

- Emily Cliff (University of Illinois at Urbana-Champaign)

- Nora Ganter (University of Melbourne)

- Arnav Tripathy (Harvard University)

- Josh Wen (University of Illinois at Urbana-Champaign) Week 2: June 9-15, 2019: Stochastic Spatial Models Organizers:

- Shankar Bhamidi (University of North Carolina, Chapel Hill)

- Gerandy Brito (Georgia Institute of Technology)

- Michael Damron (Georgia Institute of Technology)

- Rick Durrett (Duke University)

- Matthew Junge (Duke University)

Week 3: June 16-22, 2019: Explicit Methods in Arithmetic Geometry in Characteristic $p$ Organizers:

- Renee Bell (University of Pennsylvania)

- Julia Hartmann (University of Pennsylvania)

- Valentijn Karemaker (University of Pennsylvania)

- Padmavathi Srinivasan (Georgia Institute of Technology)

- Isabel Vogt (Massachusetts Institute of Technology) Individuals who are one to two years prior to receiving their PhDs or one to five years after receiving their PhDs are welcome to apply. Most of those supported by NSF funds to participate in the MRC program will be USbased, that is, employed by or full-time students at a US institution at the time of the MRC summer conference. However, the terms of the grant allow for a limited number of individuals who are not US-based. All participants are expected to be active in the full MRC program. Detailed instructions are available at www.ams.org/programs $/$ research-communities/mrc-19. Applications are due no later than February 15, 2019.

For further information on Mathematics Research Communities visit the website www. ams.org/mrc or contact Steven Ferrucci at ams-mrc@ams.org.

-Steven Ferrucci AMS Senior Program Coordinator

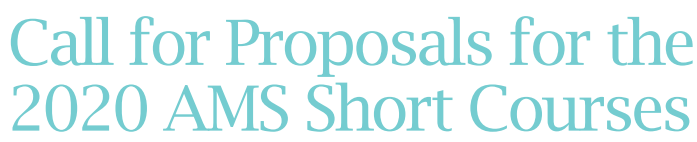

The AMS Short Course Subcommittee invites submissions of preliminary proposals for Short Courses to be offered on January 13-14, 2020, in coordination with the 2020 Joint Mathematics Meetings in Denver, Colorado. Members of the mathematical community are also welcome to suggest names of colleagues as potential organizers. 


\section{NEWS}

Preliminary proposals may be as short as one page, and suggestions and questions are welcome. Proposals should be sent via email to the Associate Executive Director (aed-mps@ams.org) with a cc to Robin Hagan Aguiar (rha@ams.org).

A short course typically incorporates a sequence of survey lectures and other activities focused on a single theme of applied mathematics. The Subcommittee is also interested in proposals that go beyond the traditional course in methodology and subject matter. Proposers might be interested in a webinar format or other mechanisms for reaching an audience that extends beyond those at the JMM site, or they may want to appeal to mathematicians who are considering careers in business, industry, government, and nonprofit sectors that utilize mathematical training and experience.

For full consideration, 2020 Short Course proposals should be submitted by December 18, 2018. More detailed guidance on proposals is available at www. ams.org/meetings /short-courses/2019ca11.

\section{Mathematical Sciences Research Institute, Berkeley,} CA

MSRI invites applications for Research Members and Postdoctoral Fellows in the following programs: Holomorphic Differentials in Mathematics and Physics (August 12-December 13, 2019), Microlocal Analysis (August 12-December 13, 2019), Quantum Symmetries (January 21-May 29, 2020) and Higher Categories and Categorification (January 21-May 29, 2020). Research Memberships are intended for researchers who will be making contributions to a program and who will be in residence for one or more months. Postdoctoral Fellowships are intended for recent PhDs.

MSRI uses MathJobs to process applications. Interested candidates must apply online beginning August 1, 2018. To receive full consideration, applications must be complete, including all letters of support, by December 1, 2018. Application information can be found at www.msri.org /application.

It is the policy of MSRI actively to seek to achieve diversity in its programs and workshops. Thus, a strong effort is made to remove barriers that hinder equal opportunity, particularly for those groups that have been historically underrepresented in the mathematical sciences.

MSRI has been supported from its origins by the National Science Foundation, now joined by the National Security Agency, over 100 Academic Sponsor departments, by a range of private foundations, and by generous and far-sighted individuals.

-MSRI announcement

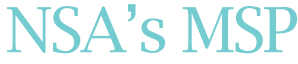

The National Security Agency's Mathematical Sciences Program (MSP) invites proposals from Principal Investigators who are seeking grants to support Research Experiences for Undergraduates, conferences, and other special events that will take place during calendar years 2019 and 2020. The MSP will accept proposals for these activities during the timeframe September 1, 2018 to October 15, 2018. Proposals must be submitted via email to the address MSPgrants@nsa.gov. PIs who plan to submit proposals should also follow the guidelines available on the NSA website (https://www.nsa.gov/what-we-do/research /math-sciences-program).

Questions may be directed to Charles Toll (chto11 ansa.gov) or Barbara Johnson (bajohn1ansa.gov).

-NSA announcement

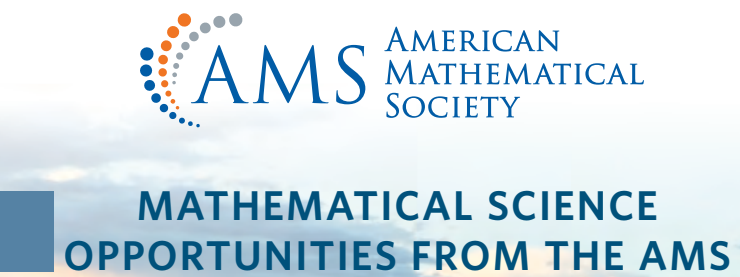

The AMS Online Opportunities Page provides another avenue for the math community to Announce and Browse:

- Calls for fellowship appointments

- Prize and award nominations

Grant applications

- Meeting and workshop proposals search now! 


\section{Call For Suggestions}

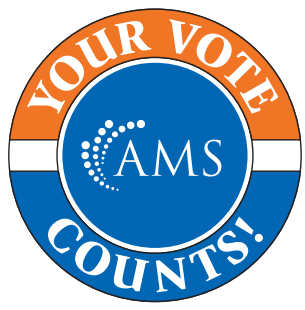

YOUR SUGGESTIONS ARE WANTED BY:

the Nominating Committee, for the following contested seats in the 2019 AMS elections:

vice president, trustee, and five members at large of the Council.

Deadline for suggestions: November 1, 2018

the President, for the following contested seats in the $\mathbf{2 0 1 9}$ AMS elections:

three members of the Nominating Committee and two members of the Editorial Boards Committee.

Deadline for suggestions: January 31, 2019

the Editorial Boards Committee, for appointments to various editorial boards of AMS publications.

Deadline for suggestions: Can be submitted any time

Send your suggestions for any of the above to:

Carla D. Savage, Secretary

American Mathematical Society

Department of Computer Science

North Carolina State University

Raleigh, NC 27695-8206 USA

secretary@ams.org

or submit them online at www.ams.org/committee-nominate 


\section{Inside the AMS}

\section{Announcements from the AMS Office of Government Relations}

\section{Next Steps in the Evolution of Mathematics Education: Moving Beyond Pilots}

Over the last decade, mathematicians have engaged in many innovations in mathematics education and, more generally, in STEM education. These can be viewed as pilots for larger-scale changes in the teaching and learning of mathematics that are now taking off nationally. On October 12, 2018, the Committee on Education of the AMS will host a mini-conference on education in Washington, DC. The conference will provide an opportunity for these changes to be discussed among mathematicians, experts from adjacent fields, and legal experts focused on the intersection between math education and civil rights. Confirmed speakers include:

- Christopher Edley (President, Opportunity Institute; Professor of Law, UC Berkeley)

- Ellen Hildreth (Professor of Computer Science, Wellesley College)

- Brit Kirwan (Retired Chancellor of the University of Maryland System, Executive Director, TPSE Math)

- Sonin Kwon (Managing Director, Investment Management Group, Mass Mutual)

- Rachel Levy (Deputy Executive Director, Mathematical Association of America)

- Jake Steel (invited), (Chief of Staff, Office of Planning, Evaluation, and Policy Development, US Department of Education)

- Manil Suri (Mathematics Professor, University of Maryland Baltimore County, \& NYT Op-Ed contributor)

This meeting will provide mathematicians, particularly chairs, an opportunity to be informed of national trends in education that impact mathematics. Participants will be encouraged to share their experiences and make their voices heard both within and beyond the mathematical community. That communication is both important and urgent. In the past, it may have been enough for the math chair to occupy herself with the business of running a department while navigating campus politics. This is no longer the case, as demonstrated by the abrupt elimination in fall 2018 of all mathematics remediation at the twenty- three California State University (CSU) campuses. This was ordered by the CSU Chancellor's Office with one year's notice, due in part to legislative pressure. Math chairs cannot afford to be ignorant of issues like opportunity and achievement gaps, outcomes-based funding, or validation of placement and admissions criteria. It is clear in state after state that legislation and legal structures are being employed to change what happens in the classroom. It is vital that mathematicians engage in these discussions if we are to have a say in the outcomes.

The Committee on Education needs strong and wellinformed voices to inspire a healthy debate on how mathematics education will meet future needs. The discussions at the mini-conference will inform the Committee's internal discussion regarding its role and goals in the coming years. Campus teams are encouraged to coordinate their participation. We look forward to a strong turnout for the mini-conference.

There is a registration fee of $\mathbf{\$ 2 0 0}$ to help offset meeting costs. If you are interested in attending, please register by September 26, 2018 at bit. 1y/2JjKCJC.

\section{Boost Your Career in Washington}

With assignments in federal agencies, on Capitol Hill, and in the judicial branch, AAAS Science \& Technology Policy Fellows are on the front line of vital issues that impact society. The AAAS Science \& Technology Policy Fellowships (STPF) are the premier opportunity for outstanding $\mathrm{PhD}$ mathematicians, scientists, and engineers to learn first-hand about policymaking while contributing their STEM knowledge to American government.

The Fellowship is yearlong and runs from September through August. Fellows broaden their career paths while engaging with policymakers, administrators, and thought leaders. They represent a broad range of backgrounds, disciplines, and career stages and are members of a strong corps of 3,000+ policy-savvy STEM leaders across academia, government, nonprofits, and industry. Currently, there are not many mathematicians in the STPF ranks: Change that by applying to become a fellow today!

The AMS sponsors one Fellowship placement in Congress each year. Learn more at bit. 1y/2LaVL1B.

The AMS Congressional Fellow is one of about $300 \mathrm{PhD}$ scientists serving in the federal government each year, as part of the large Science \& Technology Policy Fellowship program run by the American Association for the Advance- 
ment of Science (AAAS). Applications for the AMS Congressional Fellowship and the AAAS S\&T Policy Fellowship are separate, and applying to both is recommended.

The first class of AAAS fellows was in 1973 and consisted of seven scientists hosted by the American Physical Society, the American Society of Mechanical Engineers, and the Institute for Electrical and Electronic Engineering. Today, the AAAS partners with several dozen professional associations-including the AMS-and places nearly 300 fellows each year in all branches of the federal government. Each year, roughly 30-35 fellows are sponsored by one of the AMS's sister associations to work in Congress, one fellow can be placed in the Judicial Branch, and the remaining 250 or so work in the Executive Branch. Hosting offices covet their fellows. Executive Branch fellows work in many, many agencies and not just ones mathematicians might think of, like the National Science Foundation and the Department of Defense.

Mathematician STPF alumni leverage their fellowship experience in many different ways. Our own Associate Executive Director Karen Saxe was an AMS Congressional Fellow. Fellow Carla Cotwright-Williams is a scientist at the US Department of Defense. Fellow Karoline Pershell directs research at a tech company and is Executive Director of the Association for Women in Mathematics. Fellow Boris Granovskiy works for the Congressional Research Service, the public policy group within the Library of Congress that publishes thousands of nonpartisan reports per year at the request of Congressional members. Fellow Richard Yamada serves as the Deputy Assistant Administrator for the Office of Research and Development at the EPA.

Read more about these fellowships, and about the work of the mathematicians serving in the 2017-2018 Fellowship class at bit. 7y/2LpWsHO.

Application deadline is November 1, 2018, for AAAS S\&T Policy fellowships. Read more at www.stpf-aaas. org.

Application deadline is February 15, 2019, for the AMS Congressional Fellowship. Read more at bit. 1y/2LaVL1B.

Reach out to the AMS Government Relations Office with questions: amsdc@ams.org.

\section{From the AMS Public Awareness Office}

Math in the Media is a survey of math and mathematicians in the news. Here you'll find Tony Phillips' Take and summaries of, as well as links to, articles and radio segments from Science, $\mathrm{Na}$ ture, Quanta Magazine, The Guardian, The Conversation, National Public Radio, Science News, Forbes, university news offices, and other sources. Recent topics include calculus, Alan Turing, puzzles, math history, math and taxonomy, book reviews, and much more. www.ams.org/ mathmedia.

Feature Column features essays for those who enjoy mathematics-novices and experts alike. Recent col-

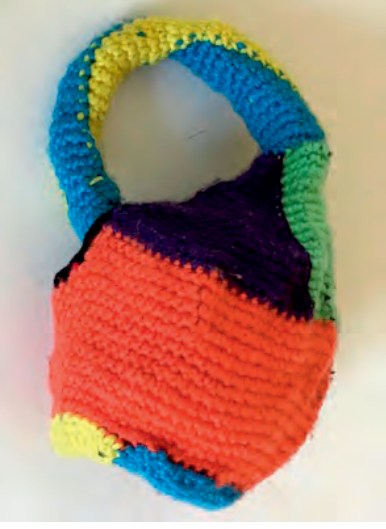

Six-color map on the Klein bottle, Moira Chas. umns include: "Reading the Bakhshali Manuscript," "Crochet Topology," "Mathematical Economics," "Neural Nets and How They Learn." www . ams . org/featurecolumn. 


\section{SCIENTIFIC OVERVIEW}

Fast acquisition and routine use of 3D data due to the advance of modern technology and computer power makes 3D description of the real world imminent and practical in many applications such as 3D modeling, virtual reality, 3D camera, 3D printing and prototyping, etc. It is increasingly important and urgent for efficient processing, analysis, and intelligent use of a large variety of 3D data. Although many advanced technologies and efficient computational tools are well developed for 2D images from acquisition to processing, analysis, understanding and learning, their counterparts for 3D modeling and shape analysis are more challenging and less developed. From a mathematical point of view, image space is linear since it can be simply regarded as a space of two variable functions defined on a rectangular domain sampled by a uniform grid in practice. However, shape space is nonlinear and shape geometry is more challenging to represent, characterize and analyze. This makes higher-level tasks for shape analysis and understanding even more challenging. Beyond $3 \mathrm{D}$ shapes, understanding and learning geometric structures for data in high dimensional spaces is also of great importance in practice.

Recently, a lot of progress has been made in developing computational models and tools based on geometric theory. In particular, these developments provide computational techniques for extracting local and global intrinsic features and structures that are invariant under various transformations or embeddings. On the other hand, recent advances in machine learning, supervised or non-supervised, can be very effective in learning robust and distinctive features and used for data or application specific tasks such as recognition and classification. The goals of this program are to (1) further advance mathematical and computational techniques for 3D modeling and shape analysis, (2) design effective problem specific approaches combining geometry and machine learning, i.e., learning geometry from geometry, (3) generalize our understandings and techniques for shape analysis to geometric data analysis in higher dimensions.

\section{WORKSHOP SCHEDULE}

- $\quad$ Opening Day : March 11, 2019.

- Tutorials Workshop : March 12-15, 2019.

- Workshop I: Geometric Processing : April 1-5, 2019.

- $\quad$ Workshop II: Shape Analysis : April 15-19, 2019.

- Workshop III: Geometry of Big Data : April 29 - May 3, 2019.

- Workshop IV: Deep Geometric Learning of Big Data and Applications : May 20-24, 2019.

- $\quad$ Culminating Workshop at Lake Arrowhead Conference Center : June 9-14, 2019.

\section{PARTICIPATION}

This long program will involve senior and junior researchers from several communities relevant to this program. You may apply for financial support to participate in the entire fourteen-week program, or a portion of it. We prefer participants who stay for the entire program. Applications will be accepted through Decembar 11, 2019, but offers may be made up to one year before the start date. We urge you to apply early. Mathematicians and scientists at all levels who are interested in this area of research are encouraged to apply for funding. Supporting the careers of women and minority researchers is an important component of IPAM's mission, and we welcome their applications.

\section{www.ipam.ucla.edu/gl2019}

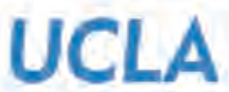




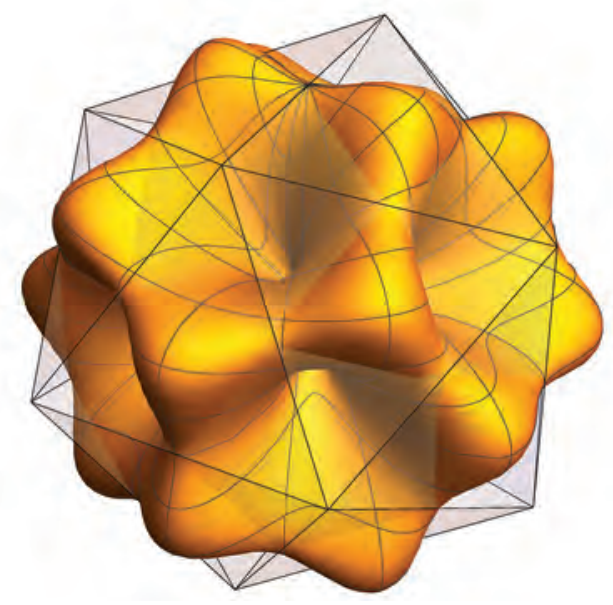

\section{Introduction}

Interest in the theory and application of sums of squares (SOS) polynomials has exploded in the last two decades, spanning a wide spectrum of mathematical disciplines from real algebraic geometry to convex geometry, combinatorics, real analysis, theoretical computer science, quantum information and engineering. This two-day short course, organized by Pablo Parrilo, Massachusetts Institute of Technology, and Rekha Thomas, University of Washington, will offer six introductory lectures on the theory of SOS polynomials and their applications. The six speakers represent a broad and diverse cross-section of the many aspects of sum of squares techniques and their interconnections.

The origins of SOS polynomials are anchored in the 19th century by Hilbert's famous characterization of nonnegative polynomials that are SOS. In 1924 Artin gave an affirmative answer to Hilbert's 17th problem on whether all nonnegative polynomials were SOS of rational functions. From this the field of real algebraic geometry was born-the study of real solutions to polynomial systems. While real solutions of polynomials equations are considerably more complicated than their complex counterpart, their role in applications cannot be overstated. SOS polynomials have experienced a renaissance in the last few years following the work of Shor, Nesterov, Lasserre, and Parrilo that connected them to modern optimization via semidefinite programming. An active interdisciplinary community now exists around SOS polynomials with a variety of conferences and research programs at many of the top institutions worldwide. While the theory is rich and fascinating with many open questions, the wide array

For permission to reprint this article, please contact: reprint-permission@ams.org.

\section{AMS Short Course Sum of Squares: Theory and Applications}

\section{Hilton Baltimore, Baltimore, $M D$}

January 14-15, 2019

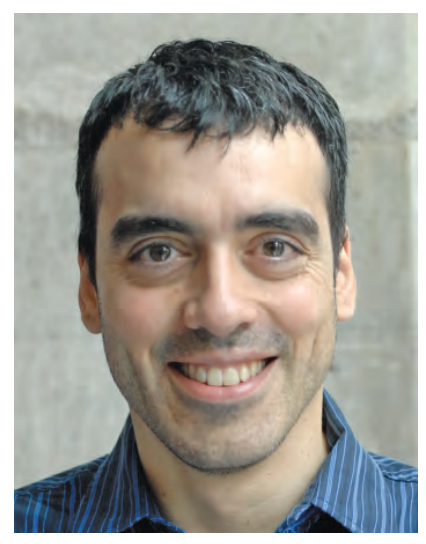

Pablo Parrilo, MIT

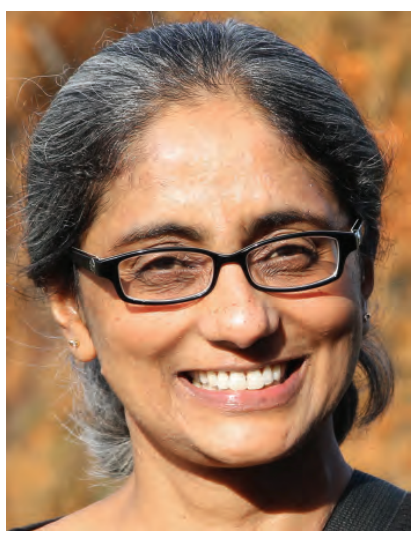

Rekha Thomas, University of Washington of applications are equally enticing, allowing for many different angles and access points to the field.

\section{Logistics}

Each of the six lectures in the course will be broken into two 45-minute sections. Problem sessions are planned for both days, and speakers and organizers will be available to assist participants with these exercises. Some exercises will be facilitated by numerical software such as Macaulay2, Maple ${ }^{\mathrm{TM}}$, MATLAB, or Julia. Participants with a desire to experiment with computations and an interest in applications should find this experience especially stimulating. The speakers will also provide participants with a list of research questions to guide their explorations after the course.

This short course is aimed at students with minimal to no background in the theory of SOS polynomials. However, familiarity with properties of polyhedra, convex sets, and polynomials will provide useful background. Appendix $\mathrm{A}$ in the book Semidefinite Optimization and Convex Al- 
gebraic Geometry, G. Blekherman, P.A. Parrilo and R.R. Thomas (eds.), MOS-SIAM Series on Optimization, SIAM 2012, is recommended as useful preparatory reading. A free pdf of the book can be found at www.mit.edu / parri1o/sdocag.

\section{Speakers and Lecture Topics}

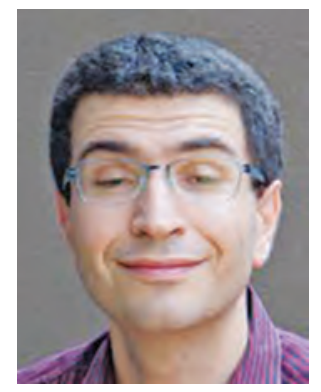

Grigoriy

Blekherman,

Georgia Institute of

Technology

applications in engineering and the-

oretical computer science. There is also an emergent understanding that the study of nonnegative and SOS polynomials on a variety is inextricably linked to classical topics in algebraic geometry and commutative algebra, such as minimal free resolutions.

This exciting blend of ideas can be demonstrated with a single prominent example: the cone of positive semidefinite (PSD) matrices. On the one hand this cone can be viewed as the set of all homogeneous quadratic polynomials (forms) nonnegative on all of $\mathbb{R}^{n}$, while on the other hand it is also a convex cone in the vector space of real symmetric matrices. It is also known that quadratic forms nonnegative on $\mathbb{R}^{n}$ are always SOS. An affine linear slice of the PSD cone is called a spectrahedron. The object of semidefinite programming is optimization of a linear function over a spectrahedron, and it is the engine that makes SOS algorithms work. SOS algorithms naturally lead to spectrahedra via convex duality, and understanding algebraic and convexity properties of these spectrahedra is the key to understanding the quality of these algorithms.

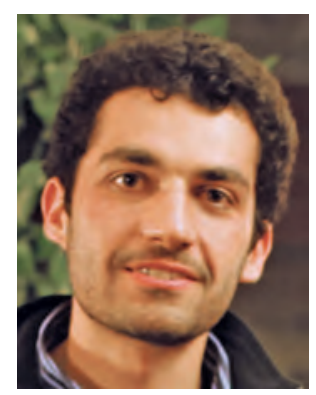

Hamza Fawzi,

University of

Cambridge

\section{Lifts of Convex Sets}

A central question in optimization is to maximize (or minimize) a linear function on a given convex set. Such a problem may be easy or hard depending on the geometry of the convex set. Motivated by this problem, this lecture considers the following question: given a convex set, is it possible to express it as the projection of a simpler convex set in a higher-dimensional space? Such a lift of the convex set allows us to reformulate the original optimization problem as an easier one over the higher-dimensional convex set. In order to make this question precise we need a way to measure the complexity of convex sets. We will focus in this lecture on two classes of lifts, namely polyhedral and spectrahedral lifts, where a natural notion of complexity can be defined. For spectrahedral lifts, we will see that the existence of lifts is characterized by the existence of SOS certificates for a certain class of nonnegative functions. We will give some examples of convex sets that admit small lifts, and others that do not, and will discuss applications in optimization.

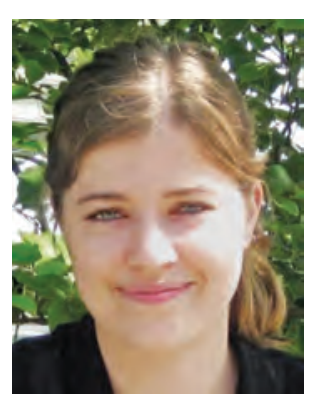

Georgina Hall,

Princeton University

\section{Engineering applications of} SOSpolynomials

The problem of optimizing over the cone of nonnegative polynomials is a fundamental problem that appears in many different areas of engineering and computational mathematics. Long thought to be intractable, several breakthrough papers in the early 2000s showed that this problem could be tackled of optimization problems which is intimately connected to semidefinite programming.

In this lecture, we present a number of problems where the need to optimize over the cone of nonnegative polynomials arises and discuss how they can be reformulated using SOS programming. Problems of this type include, but are not limited to, problems in power engineering (e.g., the optimal power flow problem) control and robotics (e.g., formal safety verification), machine learning and statistics (e.g., shape-constrained regression), and game theory (e.g., Nash equilibria computation). We conclude by highlighting some directions that could be pursued to further disseminate these techniques within more applied fields. Among other things, we address the current challenge that scalability represents for SOS programming and discuss recent trends in software development.

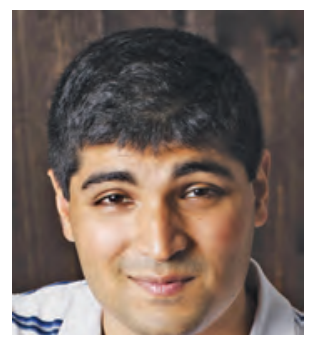

Ankur Moitra, MIT

\section{Connections to theoretical computer science}

Many hard optimization problems-like finding large cliques in a graph-can be cast as maximizing a linear function over a convex, but highly complicated domain. For example, if the feasible region is a polytope it often has an exponential number of vertices and facets without an obvious way to decide if a given point is contained inside. The SOS hierarchy gives a sequence of tighter and tighter relaxations. Each of them can be efficiently optimized over, and so it yields a sequence of algorithms that trade off complexity with accuracy. However in many cases, understanding exactly how powerful these algorithms are turns out to be quite challenging.

In this survey, we will discuss two central problems in theoretical computer science: (1) In the planted clique 
problem, the goal is to find a large clique that has been added to a random graph, and has recently found important applications in establishing computational vs. statistical tradeoffs in machine learning. (2) The unique games conjecture asserts that it is hard to find an assignment that satisfies many clauses in certain two-variable constraint satisfaction problems. If true, it would characterize the best possible approximation ratio achievable by efficient algorithms for a broad class of problems. We will explore how a deeper understanding of the power of SOS proofs has been intertwined with progress on these questions.

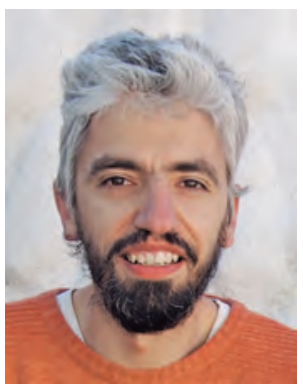

Algebraic geometrythrough the lens of sums of squares SOS optimization has a wealth of connections to pure mathematics allowing us to define new invariants of real projective varieties and to connect algebraic geometry, convexity and optimization. This lecture will illustrate these connections by focusing on a basic example: (*) the classification of those (real, projecMauricio Velasco Universidad de los Andes tive) varieties on which nonnegative quadratic forms and sums-ofsquares of linear forms coincide.

In the first part of the lecture, we will give a self-contained introduction to projective algebraic geometry and describe the classification of "varieties of minimal degree", one of the great achievements of the Italian school of algebraic geometry in the XIX century. In the second part of the lecture we will explain why varieties of minimal degree play a central "extremal" role in the theory of SOS on varieties and are the building blocks for solving problem $\left(^{*}\right)$ above. The solution of $(*)$ allows us to generalize and synthesize all known results of equality between nonnegative polynomials and sums of squares (by Choi-Lam-Reznick, Grone, Hilbert, Yakubovich, among others).

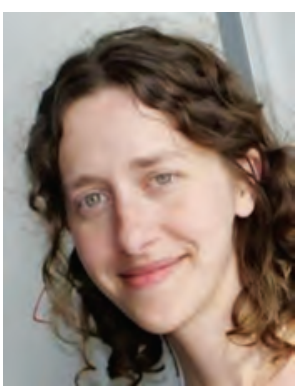

\section{The geometry of} spectrahedra

A spectrahedron is an affine slice of the convex cone of positive semidefinite real symmetric matrices. Spectrahedra form a rich class of convex bodies that are computationally tractable and appear in many areas of mathematics. Examples include polytopes, ellipsoids, and more exCynthia Vinzant, North Carolina State University otic convex sets, like the convex hull of some curves. Techniques for maximizing a linear function over a spectrahedron are called semidefinite programs. These numerical polynomial-time algorithms generalize linear programs and form a powerful tool in optimization.

The geometry of spectrahedra is fundamental to the theory of semidefinite programming, just as the geometry of polyhedra is to that of linear programming. This lecture will introduce the theory of spectrahedra and describe how convex geometry, real algebraic geometry, and topology all contribute to our understanding of their intricate geometry. We will use this to explore examples and applications coming from distance geometry, moment problems, and combinatorial optimization.

\section{Registration}

This Short Course will take place on January 14-15, 2019, the Monday and Tuesday before the Joint Mathematics Meetings (JMM). Registration is done using the same form and process as for the Joint Mathematics Meetings. However, it is not necessary to register for the JMM to participate in the Short Course. The registration fees for the Short Course are: US\$124 for AMS members; US\$190 for nonmembers; and US\$72 for students/unemployed or emeritus members. These fees are in effect until December 27,2018 . If you choose to register on-site, the fees are US\$158 for AMS members; US\$225 for nonmembers; and US\$93 for students/unemployed or emeritus members.

Advanced registration starts on September 6, 2018. On-site registration will take place on Monday, January 14, 2019, at the Hilton Baltimore, exact location to be determined.

\section{Photo Credits}

Photo of Pablo Parrilo (c) MIT.

Photo of Rekha Thomas (c) MFO.

Photo of Grigoriy Blekherman by Sung Ha Kang.

Photo of Hamza Fawzi courtesy of Hamza Fawzi.

Photo of Georgina Hall by Kimberly Lipinacci.

Photo of Ankur Moitra by Kate McKenna, Crabapple Photography. Photo of Mauricio Velasco courtesy of Mauricio Velasco. Photo of Cynthia Vinzant courtesy of Cynthia Vinzant. 


\section{Classified Advertisements}

\section{Positions available, items for sale, services available, and more}

\section{CALIFORNIA}

\section{University of California, Irvine \\ Department of Mathematics Irvine, CA 92697-3875}

The Department of Mathematics at the University of California, Irvine invites applications from outstanding candidates for multiple positions, including Lecturer with Potential Security of Employment (LPSOE), Visiting Assistant Professors (VAP), and possibly tenure-track Assistant Professors. Applicants must hold a PhD.

The LPSOE series requires, in addition to excellent teaching and service, that the candidate makes outstanding and externally recognized contributions to the development of his or her specific discipline and/or of pedagogy. VAP candidates must show strong promise in research and teaching. Tenure-track Assistant Professor position candidates should have demonstrated excellence in research and teaching.

Applications are welcome at any time. The review process starts November 1, 2018 and will continue until positions are filled. Please visit www. mathjobs.org for details on positions and the application process.

The University of California, Irvine is an Equal Opportunity/ Affirmative Action Employer advancing inclusive excellence. All qualified applicants will receive consideration for employment without regard to race, color, religion, sex, sexual orientation, gender identity, national origin, disability, age, protected veteran status, or other protected categories covered by the UC nondiscrimination policy.

\section{ILLINOIS}

\section{University of Chicago \\ Department of Mathematics}

The University of Chicago Department of Mathematics invites applications for the following positions:

1. L.E. Dickson Instructor: This is open to mathematicians who have recently completed or will soon complete a doctorate in mathematics or a closely related field, and whose work shows remarkable promise in mathematical research. The initial appointment is typically up to three years with the possibility of renewal. The teaching obligation is up to four one-quarter courses per year.

2. Assistant Professor: This is open to mathematicians who are further along in their careers, typically two or three years past the doctorate. These positions are intended for mathematicians whose work has been of outstandingly high caliber. Appointees are expected to have the potential to become leading figures in their fields. The appointment is generally for three years, with the

Suggested uses for classified advertising are positions available, books or lecture notes for sale, books being sought, exchange or rental of houses, and typing services. The publisher reserves the right to reject any advertising not in keeping with the publication's standards. Acceptance shall not be construed as approval of the accuracy or the legality of any advertising.

The 2018 rate is $\$ 3.50$ per word with a minimum two-line headline. No discounts for multiple ads or the same ad in consecutive issues. For an additional $\$ 10$ charge, announcements can be placed anonymously. Correspondence will be forwarded.

Advertisements in the "Positions Available" classified section will be set with a minimum one-line headline, consisting of the institution name above body copy, unless additional headline copy is specified by the advertiser. Headlines will be centered in boldface at no extra charge. Ads will appear in the language in which they are submitted.

There are no member discounts for classified ads. Dictation over the telephone will not be accepted for classified ads.

Upcoming deadlines for classified advertising are as follows: November 2018-August 29, 2018; December 2018-September 21, 2018; January 2019October 17, 2018; February 2019-November 15, 2018; March 2019-December 17, 2018; April 2019-January 17, 2019; May 2019-February 18, 2019. US laws prohibit discrimination in employment on the basis of color, age, sex, race, religion, or national origin. "Positions Available" advertisements from institutions outside the US cannot be published unless they are accompanied by a statement that the institution does not discriminate on these grounds whether or not it is subject to US laws. Details and specific wording may be found on page 1373 (vol. 44).

Situations wanted advertisements from involuntarily unemployed mathematicians are accepted under certain conditions for free publication. Call toll-free 800-321-4AMS (321-4267) in the US and Canada or 401-455-4084 worldwide for further information.

Submission: Promotions Department, AMS, P.O. Box 6248, Providence, Rhode Island 02904; or via fax: 401-331-3842; or send email to c1assads@ams .org. AMS location for express delivery packages is 201 Charles Street, Providence, Rhode Island 02904. Advertisers will be billed upon publication. 
possibility for renewal and a teaching obligation of up to three one-quarter courses per year.

Applicants will be considered for any of the positions above which seem appropriate. Complete applications consist of (a) a cover letter, (b) a curriculum vitae, (c) three or more letters of reference, at least one of which addresses teaching ability, and (d) a description of previous research and plans for future mathematical research. Applicants are strongly encouraged to include information related to their teaching experience, such as a teaching statement or evaluations from courses previously taught, as well as an AMS cover sheet. If you have applied for an NSF Mathematical Sciences Postdoctoral Fellowship, please include that information in your application, and let us know how you plan to use it if awarded. Applications must be submitted online through www. mathjobs.org. Questions may be directed to apptsec@math.uchicago.edu. We will begin screening applications on November 1, 2018. Screening will continue until all available positions are filled.

The University of Chicago is an Affirmative Action/Equal Opportunity/Disabled/Veterans Employer and does not discriminate on the basis of race, color, religion, sex, sexual orientation, gender identity, national or ethnic origin, age, status as an individual with a disability, protected veteran status, genetic information, or other protected classes under the law. For additional information please see the University's Notice of Nondiscrimination at www .uchicago.edu/about/non_discrimination_statement/. Job seekers in need of a reasonable accommodation to complete the application process should call 773-702-7328 or email jgarza @math.uchicago.edu with their request.

\section{MASSACHUSETTS}

\section{Massachusetts Institute of Technology Cambridge, MA}

The Mathematics Department at MIT is seeking to fill positions in Pure and Applied Mathematics at the level of Assistant Professor or higher beginning July 2019 (for the 2019-2020 academic year, or as soon thereafter as possible). Appointments are based primarily on exceptional research qualifications. Appointees will be required to fulfill teaching duties and pursue their own research program. $\mathrm{PhD}$ in Mathematics or related field required by employment start date.

For more information and to apply, please visit www. mathjobs . org. To receive full consideration, submit applications by December 1, 2018. MIT is an Equal Opportunity, Affirmative Action Employer.

\section{Massachusetts Institute of Technology Cambridge, MA}

The Mathematics Department at MIT is seeking to fill positions in Pure and Applied Mathematics, and Statistics at the level of Instructor beginning July 2019 (for the 2019-2020 academic year). Appointments are based primarily on exceptional research qualifications. Appointees will be expected to fulfill teaching duties and pursue their own research program. $\mathrm{PhD}$ in Mathematics or related field required by employment start date.

For more information and to apply, please visit www. mathjobs . org. To receive full consideration, submit applications by December 1, 2018. MIT is an Equal Opportunity, Affirmative Action Employer.
NORTH CAROLINA

\section{University of North Carolina at Greensboro (UNCG) Department of Mathematics and Statistics}

The Department of Mathematics and Statistics at the University of North Carolina at Greensboro (UNCG) seeks applications for a tenure-track position in Computational Algebra, Combinatorics, or Number Theory at the Assistant Professor rank beginning August 1, 2019. Competitive applicants will have research expertise that strengthens our PhD program in Computational Mathematics. Candidates must hold or anticipate a $\mathrm{PhD}$ in mathematics or closely related discipline by August 1, 2019. Successful applicants will be expected to excel in teaching, maintain a vigorous research program, seek external research funding, and educate a diverse group of undergraduate and graduate students from various backgrounds. Application materials should be submitted electronically to https://spartantalent.uncg.edu/postings/11231. Review of applications will begin on November 15, 2018 and will continue until the position is filled. UNCG is especially proud of the diversity of its student body which is $43 \%$ ethnic minority (admissions.uncg. edu/discover-about. php). UNCG has been designated as a Minority Serving Institution by the US Department of Education. We seek to attract a diverse applicant pool for this position, especially women and members of minority groups, and we are strongly committed to increasing faculty diversity. UNCG is an $\mathrm{EOE} \mathrm{AA} / \mathrm{M} / \mathrm{F} / \mathrm{D} / \mathrm{V}$ employer.

\section{PENNSYLVANIA}

\section{The Pennsylvania State University Anatole Katok Chair in Mathematics}

The Department of Mathematics at The Pennsylvania State University is seeking a truly exceptional leader in modern dynamical systems to fill the newly endowed Anatole Katok Chair in Mathematics in the Eberly College of Science. The recipient of this prestigious chair should have a broad view of dynamics and related areas and play a leading role in activities of the recently endowed Anatole Katok Center for Dynamical Systems and Geometry. This position will be filled as a standing tenured faculty appointment. Substantial discretionary funds will be available, on a continuing basis, to support the Chair's research programs. Applicants must complete the Penn State application at https:// psu.jobs/job/80723 and must submit an application through Mathjobs.org with the following materials in order for the application to be complete: (1) Curriculum Vitae; (2) Publication List; and (3) Research Statement. Review of applicants will begin November 15, 2018 and continue until the position is filled.

To review the Annual Security Report which contains information about crime statistics and other safety and security matters and policies, please go to https://police.psu.edu/annual -security-reports, which will also explain how to request a paper copy of the Annual Security Report.

Penn State is an equal opportunity, affirmative action employer, and is committed to providing employment opportunities to all qualified applicants without regard to race, color, religion, age, sex, sexual orientation, gender identity, national origin, disability or protected veteran status.

\section{RHODE ISLAND}

\section{Brown University Mathematics Department}

The Mathematics Department at Brown University invites applications for one regular position, to begin July 1, 2019 at the 
level of Associate Professor with tenure or tenure-track Assistant Professor; exceptionally qualified senior candidates may be considered for appointment as full Professor. Preference will be given to applicants who will interact mathematically with current members of the Department. For more information see:https : // www.brown .edu/academics/math/faculty-0

Oualified individuals are requested to submit a letter of application, and a curriculum vitae online to: www . mathjobs . org.

Tenure-level applicants should include the names of precisely 5 references that would be contacted at the appropriate time by the search committee. Candidates should have an outstanding record of scholarship establishing them as international leaders in their fields; a demonstrated willingness to contribute to vitality of the department by mentoring students and interacting with colleagues; and evidence of effective and responsible classroom teaching.

Tenure-track level applicants should request no more than 5 reference letters to be uploaded by referees directly to the same site. At least one letter should address the candidate's teaching credentials. Candidates should have an excellent track record of research and teaching, and show clear potential to contribute in the future as tenured faculty members.

Applications received by October 15, 2018 will receive full consideration, but the search will remain open until the position is closed or filled. For further information or inquiries, write to: srsearch@math.brown.edu.

Brown University is committed to fostering a diverse and inclusive academic global community; as an EEO/AA employer, Brown considers applicants for employment without regard to, and does not discriminate on the basis of gender, race, protected veteran status, disability, or any other legally protected status.

\section{Brown University Mathematics Department}

J. D. Tamarkin Assistant Professorship: One or more three-year non-tenured non-renewable appointments, beginning July 1, 2019. The teaching load is one course one semester, and two courses the other semester and consists of courses of more than routine interest. Candidates are required to have received a $\mathrm{PhD}$ degree or equivalent by the start of their appointment, and they may have up to three years of prior academic and/or postdoctoral research experience.

Applicants should have strong research potential and a commitment to teaching. Field of research should be consonant with the current research interests of the department.

For full consideration, applicants must submit a curriculum vitae, an AMS Standard Cover Sheet, at least three letters of recommendation primarily focused on research, and one letter addressing teaching (possibly as part of a research letter), by November 19, 2018. (Later applications will be reviewed to the extent possible.) In addition, applicants are required to identify a Brown faculty member with similar research interests. Please submit all application materials on line at http://www. mathjobs.org. Email inquiries should be addressed to juniorsearch @math.brown.edu.

Brown University is committed to fostering a diverse and inclusive academic global community; as an EEO/AA employer, Brown considers applicants for employment without regard to, and does not discriminate on the basis of, gender, race, protected veteran status, disability, or any other legally protected status.

\section{Brown University Mathematics Department}

Lecturer: One full time position, to begin July 1, 2019. The teaching load is four courses per year (two per semester).

Candidates must have received a PhD degree in Mathematics by the start of the appointment. A minimum of three years full time teaching experience at the university level is required.

Applicants should have a strong commitment to teaching. Courses to be taught will include calculus at all levels and/ or linear algebra. The position will also involve coordinating multi-section high-enrollment courses. Other responsibilities will include the oversight and training of graduate student TAs.

For full consideration, applicants must submit a curriculum vitae, teaching statement, AMS Standard Cover Sheet, and at least three teaching references by November 19, 2018. (Later applications will be reviewed to the extent possible.) Please submit all application materials on line at www.mathjobs.org. Email inquiries should be addressed to info@math.brown.edu.

Brown University is committed to fostering a diverse and inclusive academic global community; as an EEO/AA employer, Brown considers applicants for employment without regard to, and does not discriminate on the basis of, gender, race, protected veteran status, disability, or any other legally protected status.

026

\section{UTAH}

\section{University of Utah Department of Mathematics}

The Department of Mathematics at the University of Utah invites applications for the following positions:

- Full-time tenure-track or tenured appointments at the level of Assistant, Associate, or Full Professor in all areas of mathematics.

- Full-time tenure-track or tenured appointments at the level of Assistant, Associate, or Full Professor in all areas of statistics. These positions are part of a University-wide cluster hiring effort in statistics, with particular emphasis in mathematics, computer science, and bioengineering. Successful candidates will have strong interdisciplinary interests.

- Three-year Burgess, Kollár, Tucker, and Wylie Assistant Professor Lecturer positions.

Please see our website at www.math.utah.edu/positions for information regarding available positions and application requirements. Applications must be completed through www. mathjobs .org/jobs/Utah. Review of complete applications for tenure-track positions will begin on October 5, 2018, and will continue until the positions are filled. Completed applications for postdoctoral positions received before January 1, 2019, will receive full consideration.

The University of Utah is an Equal Opportunity/Affirmative Action employer and educator. Minorities, women, veterans, and those with disabilities are strongly encouraged to apply. Veterans' preference is extended to qualified veterans. Reasonable disability accommodations will be provided with adequate notice. For additional information about the University's commitment to equal opportunity and access see:www. utah . edu/nondiscrimination/. 
AUSTRIA

\section{IST Austria \\ Assistant Professor (tenure-track) and \\ Professor Positions in Mathematics}

We invite applications in all areas of mathematics for several open positions.

Female researchers are strongly encouraged to apply.

We offer:

- Highly international and interdisciplinary research environment

- Competitive start-up package and salary

- Guaranteed, annual base funding

- Support for acquiring third-party funds

- Wide portfolio of career support

- Child-care facilities and support on campus

IST Austria (www. ist . ac . at) is an international institute dedicated to basic research and graduate education in the natural, mathematical, and computational sciences. The Institute fosters an interactive, collegial, and supportive atmosphere, sharing space and resources between research groups whenever possible, and facilitating cross-disciplinary collaborations. Our PhD program involves a multi-disciplinary course schedule and rotations in research groups. We hire scholars from diverse international backgrounds and our working language is English. The campus of IST Austria is located about $15 \mathrm{~km}$ from Vienna, being one of the most livable cities in the world.

Assistant professors receive independent group leader positions with an initial contract of six years, at the end of which they are reviewed by international peers. If the evaluation is positive, an assistant professor is promoted to a tenured professor.

Candidates for tenured positions are distinguished scientists in their respective research fields and have at least six years of experience in leading a research group.

Please apply online at: www. ist.ac.at/professor -applications

The closing date for applications is November 2, 2018.

IST Austria values diversity and is committed to equal opportunity.

\section{CHINA}

\section{Southern University of Science and Technology (SUSTech) Faculty Positions of Mathematics The Department of Mathematics}

The Department of Mathematics at Southern University of Science and Technology (SUSTech) is founded in 2015 with a dual mission of creating a first-class research and education organization for mathematics and providing service courses in support of other academic departments at SUSTech. We currently have 36 full-time faculty members, including 6 Chair Professors \& 7 Full Professors, 3 Associate Professors, 12 Assistant Professors, and 8 teaching faculty members. Research interests of the faculty members cover a broad array of Mathematics including Pure Mathematics, Computational and Applied Mathematics, Probability and Statistics, and Financial Mathematics.

\section{Call for Applications}

We invite applications for full-time faculty positions at all ranks and in all areas of Mathematics, including Financial Mathematics and Statistics. SUSTech has a tenure system. Qualified candidates may apply for appointments with tenure.

Candidates should have demonstrated excellence in research and a strong commitment to teaching. A doctoral degree is required at the time of appointment. A candidate for a senior posi- tion must have an established record of research and teaching, and a track-record in securing external funding.

To apply, please visit www. mathjobs . org and look up our job ad for instructions. For an informal discussion about applying to one of our positions, please contact Ms. Xianghui Yu, the Secretary of Department of Mathematics, by phone $+86-755-88018703$ or email: yuxh@sustc.edu.cn.

SUSTech offers competitive salaries, fringe benefits including medical insurance, retirement and housing subsidy, which are among the best in China. Salary and rank will be commensurate with qualifications and experiences of an appointee.

\section{About the University}

Established in 2012, SUSTech is a public institution funded by Shenzhen, a city with a designated special economic zone status in Southern China bordering Hong Kong. As one of China's key gateways to the world, Shenzhen is the country's fastest-growing city in the past three decades. From a small fishing village 30 years ago to a modern city with a population of over 10 million, the city has become the high-tech and manufacturing hub of southern China. It is home to the world's third-busiest container port and the fourth-busiest airport on the Chinese mainland. Being a picturesque coastal city, Shenzhen is also a popular tourist destination.

SUSTech is a pioneer in higher education reform in China. Its mission is to become a globally recognized institution that excels in research and promotes innovation, creativity and entrepreneurship. Ninety percent of SUSTech faculty members have overseas work experiences, and sixty percent studied or worked in top 100 universities in the world. The languages of instruction are English and Chinese. Sitting on five hundred acres of subtropical woodland with hills, rivers and a natural lake in Nanshan District of Shenzhen, the SUSTech campus is a beautiful place for learning and research.

The prosperity of Shenzhen is built on innovations and entrepreneurship of its citizens. The city has some of China's most successful high-tech companies such as Huawei and Tencent. SUSTech strongly supports innovations and entrepreneurship, and provides funding for promising initiatives. The university encourages candidates with intention and experience on entrepreneurship to apply.

\section{Tianjin University, China Tenured/Tenure-Track/Postdoctoral Positions at the Center for Applied Mathematics}

Dozens of positions at all levels are available at the recently founded Center for Applied Mathematics, Tianjin University, China. We welcome applicants with backgrounds in pure mathematics, applied mathematics, statistics, computer science, bioinformatics, and other related fields. We also welcome applicants who are interested in practical projects with industries. Despite its name attached with an accent of applied mathematics, we also aim to create a strong presence of pure mathematics. Chinese citizenship is not required.

Light or no teaching load, adequate facilities, spacious office environment and strong research support. We are prepared to make quick and competitive offers to self-motivated hard workers, and to potential stars, rising stars, as well as shining stars.

The Center for Applied Mathematics, also known as the Tianjin Center for Applied Mathematics (TCAM), located by a lake in the central campus in a building protected as historical architecture, is jointly sponsored by the Tianjin municipal government and the university. The initiative to establish this center was taken by Professor S. S. Chern. Professor Molin Ge is the Honorary Director, Professor Zhiming Ma is the Director of the Advisory Board. Professor William Y. C. Chen serves as the Director.

TCAM plans to fill in fifty or more permanent faculty positions in the next few years. In addition, there are a number of temporary 
and visiting positions. We look forward to receiving your application or inquiry at any time. There are no deadlines.

Please send your resume to mathjobs@tju.edu.cn.

For more information, please visit cam.tju.edu.cn or contact Ms. Erica Liu at mathjobs@tju.edu.cn, telephone: 86-22-2740-6039.

JAPAN

\section{OIST Okinawa Institute of Science and Technology Open Faculty Positions in Mathematics}

As a part of an effort to create an internationally renowned group in the mathematical sciences, OIST in Japan, invites applications for at least two tenured or tenure-track faculty positions in Pure Mathematics.

Instructions regarding application submission: https:// groups.oist.jp/facultypositions.

Application Deadline: October 15, 2018 (EDT)

Applications are strongly encouraged by August 31, 2018.

\section{SINGAPORE}

\section{National University of Singapore (NUS) Department of Mathematics}

The Department of Mathematics at the National University of Singapore (NUS) invites applications for the following positions beginning in August 2019. NUS is a leading global university centred in Asia. The Department of Mathematics has about 60 faculty members and teaching staff whose expertise cover major areas of contemporary mathematical research.

1) Tenured/tenure-track faculty positions at all levels

2) Peng Tsu Ann Assistant Professorship (non-tenure track)

\section{1) Tenured/tenure-track faculty positions at all levels}

We seek promising scholars and established mathematicians with outstanding track records in any field of pure and applied mathematics. The Department, housed in a spacious building equipped with state-of-the-art facilities, offers internationally competitive salary with start-up research grants, as well as an environment conducive to active research, and ample opportunities for career development. The teaching load for junior faculty is kept especially light.

The Department is particularly interested in, but not restricted to, considering applicants specializing in any of the following areas:

- Optimization/machine learning, quantitative finance, network modelling/discrete mathematics, with strong emphasis in data related research.

- Algebraic/arithmetic geometry

- Homogeneous dynamics

Please submit your application at https://www.mathjobs. org/jobs/jobs/12163

\section{2) Peng Tsu Ann Assistant Professorship (non-tenure} track)

The Department of Mathematics and the Institute for Mathematical Sciences at the National University of Singapore (NUS) invite applications for the position of Peng Tsu Ann Assistant Professorship (non-tenure track) beginning in August 2019. We seek promising young scholars (fresh or recent PhD's) whose research programmes synergize with research thrusts of our senior faculty members.

The position is for a two-year duration with the possibility of a one-year renewal, and it comes with subsidized university housing. The teaching load of this appointment is especially light.

Please submit your application at https://www. mathjobs. org/jobs/jobs/12164

Review process will begin on 15 October, and will continue until positions are filled. Enquiries may be sent to search@math . nus. edu.sg

For further information about the department, please visit www. math.nus.edu.sg.

\section{BULLETIN OF THE AMS}

The Bulletin publishes expository articles on contemporary mathematical research, written in a way that gives insight to mathematicians who may not be experts in the particular topic. The Bulletin also publishes reviews of selected books in mathematics and short articles in the Mathematical Perspectives section, both by invitation only.

Visit www.ams.org/bulletin to view the current issue as well as past issues going back to 1891 .
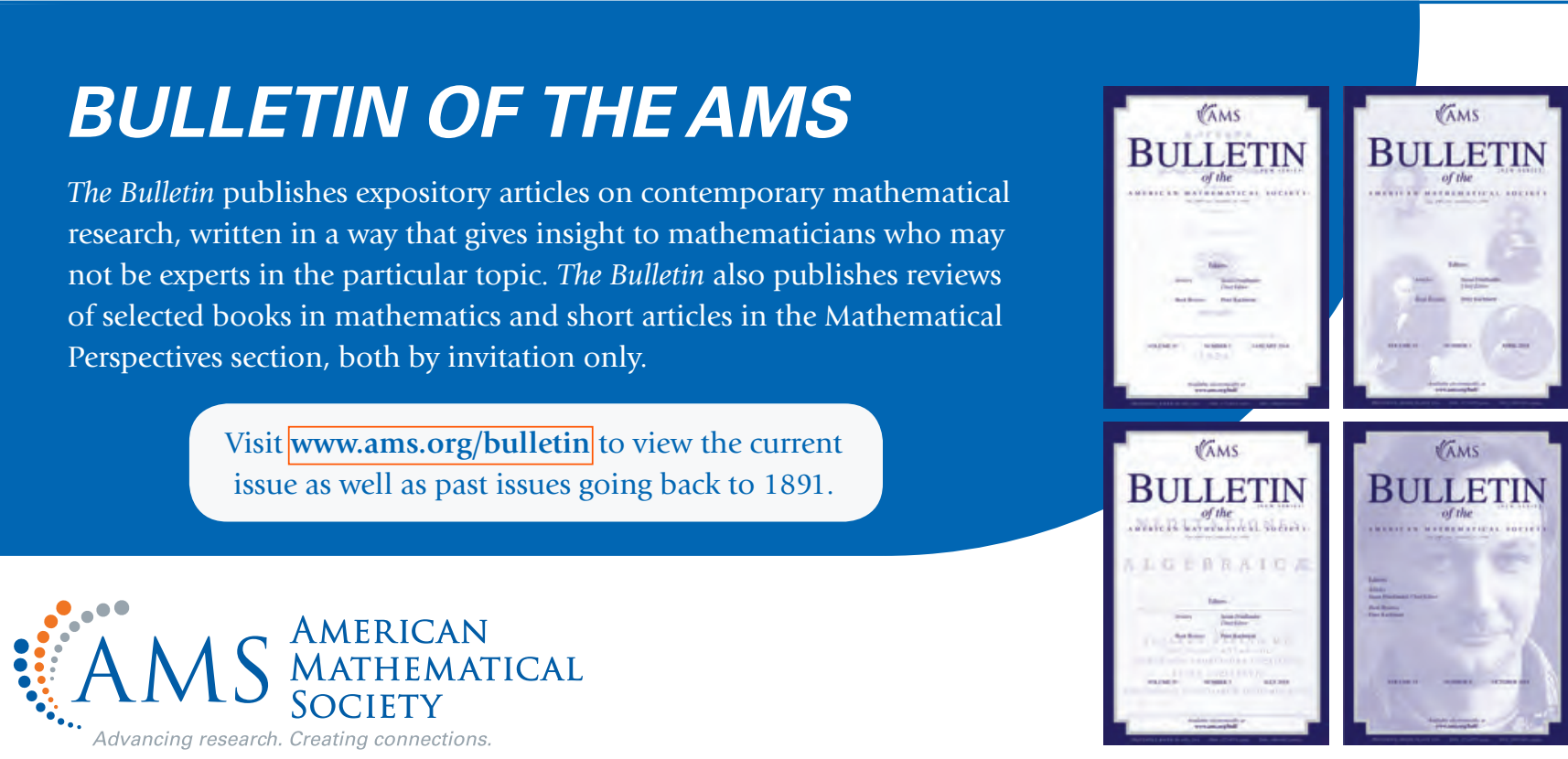


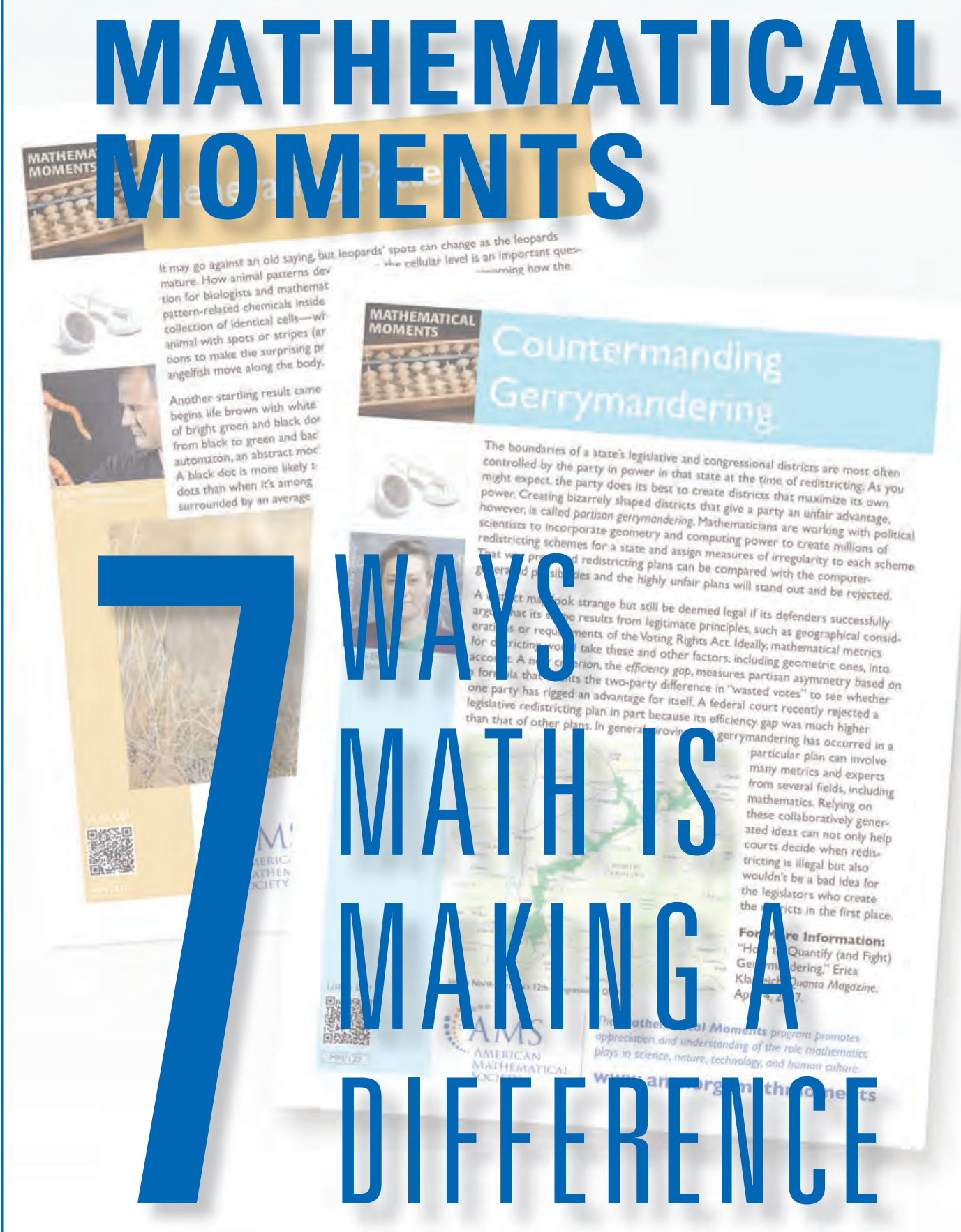

See over 100 Mathematical Moments, hear people talk about how they use math on the job in the modern world, and read translations in 13 languages at WWW.ams.org/mathmoments 


\section{New Publications Offered by the AMS}

To subscribe to email notification of new AMS publications, please go to www . ams. org/bookstore-emai 1.

\section{Algebra and Algebraic Geometry}

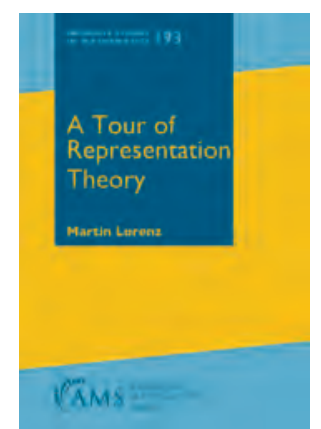

A Tour of Representation Theory

\section{Martin Lorenz, Temple University, Philadelphia, PA}

Representation theory investigates the different ways in which a given algebraic object-such as a group or a Lie algebra-can act on a vector space.

Besides being a subject of great intrinsic beauty, the theory enjoys the additional benefit of having applications in myriad contexts outside pure mathematics, including quantum field theory and the study of molecules in chemistry.

Adopting a panoramic viewpoint, this book offers an introduction to four different flavors of representation theory: representations of algebras, groups, Lie algebras, and Hopf algebras. A separate part of the book is devoted to each of these areas and they are all treated in sufficient depth to enable and hopefully entice the reader to pursue research in representation theory.

The book is intended as a textbook for a course on representation theory, which could immediately follow the standard graduate abstract algebra course, and for subsequent more advanced reading courses. Therefore, more than 350 exercises at various levels of difficulty are included. The broad range of topics covered will also make the text a valuable reference for researchers in algebra and related areas and a source for graduate and postgraduate students wishing to learn more about representation theory by self-study.

Contents: Algebras: Representations of algebras; Further topics on algebras; Groups: Groups and group algebras; Symmetric groups; Lie algebras: Lie algebras and enveloping algebras; Semisimple Lie algebras; Root systems; Representations of semisimple Lie algebras; Hopf algebras: Coalgebras, bialgebras, and Hopf algebras; Representations and actions; Affine algebraic groups; Finite-dimensional Hopf algebras; Appendices: The language of categories and functors; Background from linear algebra; Some commutative algebra; The Diamond Lemma; The symmetric ring of quotients; Bibliography; Subject index; Index of names; Notation.

Graduate Studies in Mathematics, Volume 193

October 2018, 654 pages, Hardcover, ISBN: 978-1-4704-3680-3, LC 2018016461, 2010 Mathematics Subject Classification: 16Gxx, 16Txx, 17Bxx, 20Cxx, 20Gxx, AMS members US\$75.20, List US\$94, Order code GSM/193

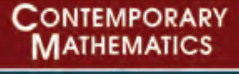

MATHEMATICS

Advances in

Rings and Modules

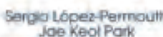

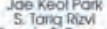

Cosming, Roman

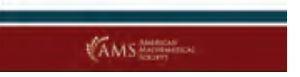

This volume, dedicated to Bruno J. Müller, a renowned algebraist, is a collection of papers that provide a snapshot of the diversity of themes and applications that interest algebraists today.

The papers highlight the latest progress in ring and module research and present work done on the frontiers of the topics discussed.

In addition, selected expository articles are included to give algebraists and other mathematicians, including graduate students, an accessible introduction to areas that may be outside their own expertise.

Contents: G. Abrams, B. Greenfeld, Z. Mesyan, and K. M. Rangaswamy, Chains of semiprime and prime ideals in Leavitt path algebras; $\mathbf{T}$. Albu, The conditions $\left(C_{i}\right), i=1,2,3,11,12$, in rings, modules, categories, and lattices; A. Y. Ammar, A. S. El-Araby, M. A. Kamal, and N. S. Mahmoud, On $K$-semi discrete modules; M. Ashraf and A. Jabeen, Nonlinear Lie triple higher derivation on triangular algebras; J. A. Beachy and C. M. Leroux, On universal localization of Noetherian rings; G. F. Birkenmeier and E. K. S. Lee, A survey of intrinsic extensions of rings; W. D. Burgess and R. Raphael, The reduced ring order and lower semi-lattices; F. Campanini and A. Facchini, On a category of extensions whose endomorphism rings have at most four maximal ideals; N. V. Dung and J. L. García, Tilting cotorsion pairs and pure semisimple rings; K. R. Goodearl and M. T. Yakimov, Twist invariants of graded algebras; P. A. Guil Asensio, D. K. Keskin Tütüncü, and A. K. Srivastava, Modules invariant under monomorphisms of their envelopes; D. V. Huynh and 
D. D. Tai, Some results and questions on left-right symmetry; A. Karimi-Mansoub, T. Koşan, and Y. Zhou, Rings in which every unit is a sum of a nilpotent and an idempotent; D. Khurana and T. Y. Lam, Commutators and anti-commutators of idempotents in rings; S. López-Permouth and L. H. Rowen, Distributive hierarchies of binary operations; H. Marubayashi and A. Ueda, Idealizers in differential polynomial rings and generalized HNP rings; J. K. Park and S. T. Rizvi, Module hulls-similarities and contrasts; S. Singh, Direct sums of completely almost self-injective modules.

Contemporary Mathematics, Volume 715

October 2018, approximately 283 pages, Softcover, ISBN: 978-14704-3555-4, LC 2018007403, 2010 Mathematics Subject Classification: 16Dxx, 16Sxx, 16Wxx, 16Exx, 16Gxx, 16Nxx, 16Pxx, 16Uxx, 06Cxx, 15Bxx, AMS members US\$93.60, List US\$117, Order code $\mathrm{CONM} / 715$

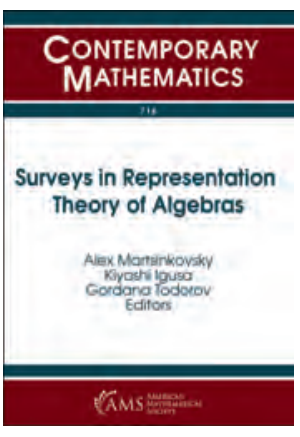

Surveys in Representation Theory of Algebras
Alex Martsinkovsky, Northeastern University, Boston, $M A$, Kiyoshi Igusa, Brandeis University, Waltham, MA, and Gordana Todorov, Northeastern University, Boston, MA, Editors

This volume contains selected expository lectures delivered at the annual Maurice Auslander Distinguished Lectures and International Conference over the last several years.

Reflecting the diverse landscape of modern representation theory of algebras, the selected articles include: a quick introduction to silting modules; a survey on the first decade of co-t-structures in triangulated categories; a functorial approach to the notion of module; a representation-theoretic approach to recollements in abelian categories; new examples of applications of relative homological algebra; connections between Coxeter groups and quiver representations; and recent progress on limits of approximation theory.

Contents: L. Angeleri Hügel, On the abundance of silting modules; P. Jørgensen, Co-t-structures: The first decade; M. Prest, Modules as exact functors; C. Psaroudakis, A representation-theoretic approach to recollements of abelian categories; Ø. Solberg, Going relative with Maurice-A survey; $\mathbf{H}$. Thomas, Coxeter groups and quiver representations; J. Trlifaj, Tree modules and limits of the approximation theory.

Contemporary Mathematics, Volume 716

October 2018, 203 pages, Softcover, ISBN: 978-1-4704-3679-7, LC 2018018535, 2010 Mathematics Subject Classification: 16E30, 18E30, 18E10, 16G99, 18G25, 16G20, 16G70, AMS members US\$93.60, List US\$117, Order code CONM/716

\section{Analysis}

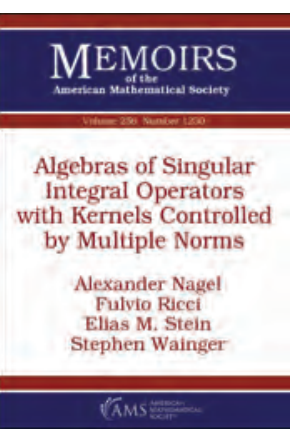
Algebras of Singular
Integral Operators with Kernels Controlled by Multiple Norms

\author{
Alexander Nagel, University of \\ Wisconsin-Madison, WI, Fulvio \\ Ricci, Scuola Normale Superiore, \\ Pisa, Italy, Elias M. Stein, \\ Princeton University, NJ, and \\ Stephen Wainger, University of \\ Wisconsin-Madison, WI
}

Contents: Introduction; The Classes $\mathcal{P}(\mathrm{E})$ and $\mathcal{M}(\mathbf{E})$; Marked partitions and decompositions of $\mathbb{R}^{N}$; Fourier transform duality of kernels and multipliers; Dyadic sums of Schwartz functions; Decomposition of multipliers and kernels; The rank of $\mathbf{E}$ and integrability at infinity; Convolution operators on homogeneous nilpotent Lie groups; Composition of operators; Convolution of Calderón-Zygmund kernels; Two-flag kernels and multipliers; Extended kernels and operators; The role of pseudo-differential operators; Appendix I: Properties of cones $\Gamma(\mathbf{A})$; Appendix II: Estimates for homogeneous norms; Appendix III: Estimates for geometric sums; Bibliography.

Memoirs of the American Mathematical Society, Volume 256, Number 1230

October 2018, 141 pages, Softcover, ISBN: 978-1-4704-3438-0, 2010 Mathematics Subject Classification: 42B20, 35S05, Individual member US\$46.80, List US\$78, Institutional member US\$62.40, Order code MEMO/256/1230

\section{Differential Equations}

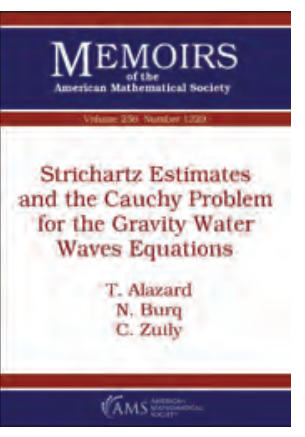

Strichartz Estimates and the Cauchy Problem for the Gravity Water Waves Equations

\section{T. Alazard, École Normale Supérieure, Paris, France, N. Burq, Université Paris-Sud, Orsay, France, and C. Zuily, Université Paris-Sud, Orsay, France}

This item will also be of interest to those working in applications. Contents: Introduction; Strichartz estimates; Cauchy problem; Appendix A. Paradifferential calculus; Appendix B. Tame estimates 
for the Dirichlet-Neumann operator; Appendix C. Estimates for the Taylor coefficient; Appendix D. Sobolev estimates; Appendix E. Proof of a technical result; Bibliography.

Memoirs of the American Mathematical Society, Volume 256, Number 1229

October 2018, 108 pages, Softcover, ISBN: 978-1-4704-3203-4, 2010 Mathematics Subject Classification: 35Q35, 35S50, 35S15, $76 \mathrm{~B} 15$, Individual member US\$46.80, List US\$78, Institutional member US\$62.40, Order code MEMO/256/1229

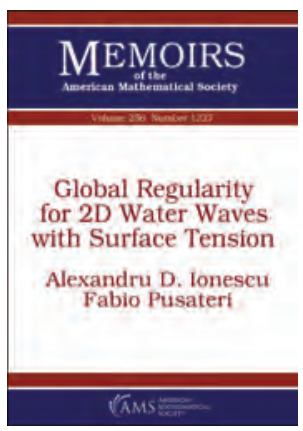

\section{Global Regularity for 2D Water Waves with Surface Tension}
Alexandru D. Ionescu, Princeton
University, NJ, and Fabio
Pusateri, Princeton University, NJ

This item will also be of interest to those working in applications.

Contents: Introduction; Preliminaries; Derivation of the main scalar equation; Energy estimates I: high Sobolev estimates; Energy estimates II: low frequencies; Energy estimates III: weighted estimates for high frequencies; Energy estimates IV: weighted estimates for low frequencies; Decay estimates; Proof of Lemma 8.6; Modified scattering; Appendix A. Analysis of symbols; Appendix B. The Dirichlet-Neumann operator; Appendix C. Elliptic bounds; Bibliography.

Memoirs of the American Mathematical Society, Volume 256, Number 1227

October 2018, 119 pages, Softcover, ISBN: 978-1-4704-3103-7, 2010 Mathematics Subject Classification: 76B15; 35Q35, Individual member US\$46.80, List US\$78, Institutional member US\$62.40, Order code MEMO/256/1227

\section{Discrete Mathematics and Combinatorics}

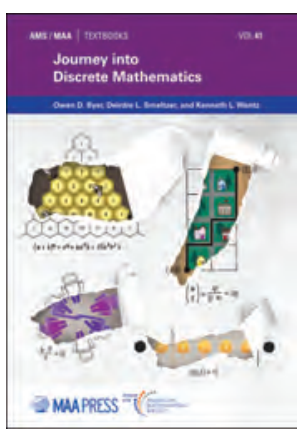

\section{Journey into Discrete Mathematics}

\author{
Owen D. Byer, Eastern \\ Mennonite University, \\ Harrisonburg, VA, Deirdre L. \\ Smeltzer, Eastern Mennonite \\ University, Harrisonburg, VA, \\ and Kenneth L. Wantz, Regent \\ University, Virginia Beach, VA
}

Journey into Discrete Mathematics is designed for use in a first course in mathematical abstraction for early-career undergraduate mathematics majors. The important ideas of discrete mathematics are included-logic, sets, proof writing, relations, counting, number theory, and graph theory-in a manner that promotes development of a mathematical mindset and prepares students for further study. While the treatment is designed to prepare the student reader for the mathematics major, the book remains attractive and appealing to students of computer science and other problem-solving disciplines.

The exposition is exquisite and engaging and features detailed descriptions of the thought processes that one might follow to attack the problems of mathematics. The problems are appealing and vary widely in depth and difficulty. Careful design of the book helps the student reader learn to think like a mathematician through the exposition and the problems provided. Several of the core topics, including counting, number theory, and graph theory, are visited twice: once in an introductory manner and then again in a later chapter with more advanced concepts and with a deeper perspective.

Owen D. Byer and Deirdre L. Smeltzer are both Professors of Mathematics at Eastern Mennonite University. Kenneth L. Wantz is Professor of Mathematics at Regent University. Collectively the authors have specialized expertise and research publications ranging widely over discrete mathematics and have over fifty semesters of combined experience in teaching this subject.

Contents: Convince me!; Mini-theories; Logic and sets; Logic and proof; Relations and functions; Induction; Number theory; Counting; Graph theory; Invariants and monovariants; Topics in counting; Topics in number theory; Topics in graph theory; Hints; List of names; Bibliography; Index.

AMS/MAA Textbooks, Volume 41

December 2018, approximately 389 pages, Hardcover, ISBN: 978-1-4704-4696-3, LC 2018023584, 2010 Mathematics Subject Classification: 97K20, 97K30, 97F60, 97N70, 97E30, Individual member US\$56.25, List US\$75, Institutional member US\$60, Order code TEXT/41

\section{General Interest}

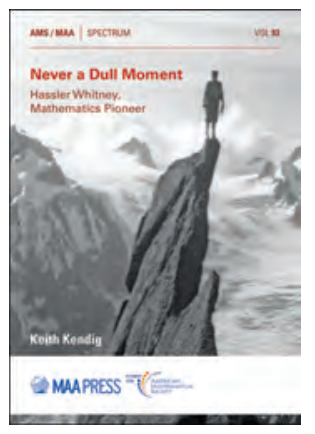

Never a Dull Moment

Hassler Whitney, Mathematics Pioneer

\author{
Keith Kendig, Cleveland State \\ University, $\mathrm{OH}$
}

Hassler Whitney was a giant of twentieth-century mathematics. This biography paints a picture of him and includes dozens of revealing anecdotes. Mathematically, he had a rare detector that went off whenever he spotted a piece of mathematical gold, and he would then draw countless pictures, gradually forging a path from hunch to proof. This geometric path is seldom reflected in the rigor of his formal papers, but thanks to a close friendship and many conversations over decades, author Kendig was able to see how he actually worked. This book shows this through accessible accounts of his major mathematical contributions, with figures copiously supplied.

Whitney is probably best known for introducing the grandfather of today's innumerable embedding theorems-his strong embedding theorem stating that any smooth manifold can be smoothly embedded in a Euclidean space of twice the manifold's dimension. 
This in turn led to several standard techniques used every day in algebraic topology. Whitney also established the fundamentals of graph theory, the four-color problem, matroids, extending smooth functions, and singularities of smooth functions. He almost never used complicated technical machinery, so most of his work is accessible to a general reader with a modest mathematical background.

His math-music connection was intense: He played piano, violin, and viola and won "best composition of the year" while earning a Bachelor's degree in music at Yale. He was an accomplished mountain climber, and as a tinkerer, at age sixteen he built the large-format camera used to take this book's cover photograph. Whitney's family generously provided dozens of photographs appearing here for the very first time. This biography is a revealing portrait of a fascinating personality and a titan of twentieth-century mathematics.

Contents: Permissions; Some snapshots; How Hassler chose his genes; Growing up; Hassler goes to college; Early days at Harvard; The four-color problem: Some history and Whitney's contributions to it; Whitney and the four-color problem: A closer look; Whitney discovers a big brother to the matrix: The matroid; Topology: Its beginnings; Topology grows into a branch of mathematics; Whitney helped revolutionize algebraic topology; Whitney's extension theorems; Whitney's weak embedding theorem; Whitney's strong embedding theorem; World War II; From Harvard to the Institute, and insights on smooth mappings; Are there decomposition theorems for nonmanifolds?; After research; Evolution or revolution?; Other happenings at the Institute; The unspeakable was about to happen; Sometimes you get to know people through the little things; Parting shots: A gallery of photos; Notes; Bibliography; Index.

Spectrum, Volume 93

November 2018, approximately 381 pages, Softcover, ISBN: 978-1-4704-4828-8, LC 2018023363, 2010 Mathematics Subject Classification: 01-XX, 05C10, 57-XX, 58Axx, 37C05, 57N80, 97Dxx, Individual member US\$45, List US\$60, Institutional member US $\$ 48$, Order code SPEC $/ 93$

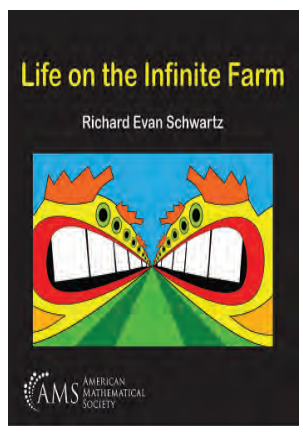

\section{Life on the Infinite Farm}

Richard Evan Schwartz, Brown University, Providence, RI

Pay a visit to the Infinite Farm!

In Life on the Infinite Farm, mathematician and award-winning children's book author Richard Schwartz teaches about infinity and curved space through stories of whimsical farm animals. Join Gracie, the shoe-loving cow with infinitely many feet, Hammerwood, the gum-loving crocodile with an endless mouth, and their friends as they navigate the challenges that come with being infinitely large.

Children as young as 5 will enjoy the lighthearted illustrations and the fanciful approach to infinity. Older students (and even adult professional mathematicians) will also appreciate the more

advanced ideas and geometric references. The two approaches are woven together to appeal to a wide audience, from budding mathematicians to hardcore geometers.

October 2018, 176 pages, Softcover, ISBN: 978-1-4704-4736-6, LC 2018017358, 2010 Mathematics Subject Classification: 97A80, AMS members US\$20, List US\$25, Order code MBK/115

\section{Geometry and Topology}
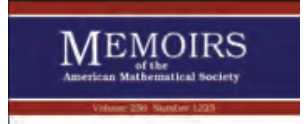

Curvature:

A Variational Approach

A. Agrachev

D. Barilar

L. Rizzi

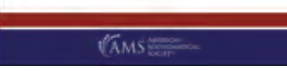

Curvature: A Variational Approach

A. Agrachev, SISSA, Trieste, Italy, and Sobolev Institute of Mathematics, Novosibirsk, Russia, D. Barilari, École Polytechnique, Paris, France, and INRIA GECO Saclay-Ile-de-France, Paris, France, and L. Rizzi, SISSA, Trieste, Italy

Contents: Introduction; Part 1. Statements of the results: General setting; Flag and growth vector of an admissible curve; Geodesic cost and its asymptotics; Sub-Riemannian geometry; Part 2. Technical tools and proofs: Jacobi curves; Asymptotics of the Jacobi curve: equiregular case; Sub-Laplacian and Jacobi curves; Part 3. Appendices: Appendix A. Smoothness of value function (Theorem 2.19); Appendix B. Convergence of approximating Hamiltonian systems (Proposition 5.15); Appendix C. Invariance of geodesic growth vector by dilations (Lemma 5.20); Appendix D. Regularity of $C(t, s)$ for the Heisenberg group (Proposition 5.51); Appendix E. Basics on curves in Grassmannians (Lemmas 3.5 and 6.5); Appendix F. Normal conditions for the canonical frame; Appendix G. Coordinate representation of flat, rank 1 Jacobi curves (Proposition 7.7); Appendix H. A binomial identity (Lemma 7.8); Appendix I. A geometrical interpretation of $\dot{c}_{t}$; Bibliography; Index.

Memoirs of the American Mathematical Society, Volume 256, Number 1225

October 2018, 116 pages, Softcover, ISBN: 978-1-4704-2646-0, 2010 Mathematics Subject Classification: 49-02, 53C17, 49J15, 58B20, Individual member US\$46.80, List US\$78, Institutional member US\$62.40, Order code MEMO/256/1225 


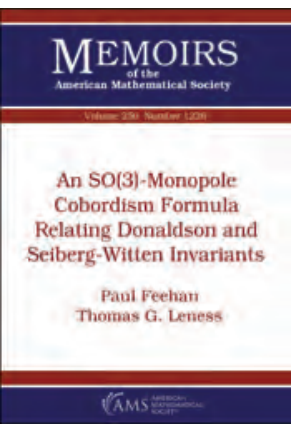

An SO(3)-Monopole Cobordism Formula Relating Donaldson and Seiberg-Witten Invariants

Paul Feehan, Rutgers, The State University of New Jersey, Piscataway, NJ, and Thomas G. Leness, Florida International University, Miami, FL

Contents: Preface; Introduction; Preliminaries; Diagonals of symmetric products of manifolds; A partial Thom-Mather structure on symmetric products; The instanton moduli space with spliced ends; The space of global splicing data; Obstruction bundle; Link of an ideal Seiberg-Witten moduli space; Cohomology and duality; Computation of the intersection numbers; Kotschick-Morgan conjecture; Glossary of notation; Index; Bibliography.

Memoirs of the American Mathematical Society, Volume 256, Number 1226

October 2018, 228 pages, Softcover, ISBN: 978-1-4704-1421-4, 2010 Mathematics Subject Classification: 57N13, 57R57, 58D27, 58D29; 53C07, 53C27, 58J05, 58J20, Individual member US\$46.80, List US\$78, Institutional member US\$62.40, Order code MEMO/256/1226

\section{Mathematical Physics}

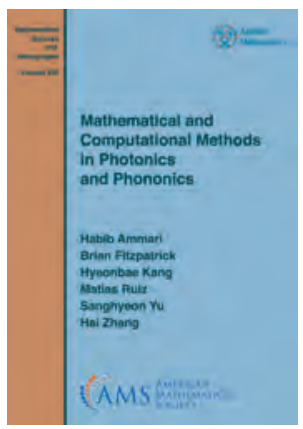

Mathematical and Computational Methods in Photonics and Phononics

Habib Ammari, ETH, Zürich, Switzerland, Brian Fitzpatrick, ETH, Zürich, Switzerland, Hyeonbae Kang, Inha University, Incheon, Korea, Matias Ruiz, École Normale Supérieure, Paris, France, Sanghyeon Yu, ETH, Zürich, Switzerland, and Hai Zhang, HKUST, Clear Water Bay, Hong Kong

The fields of photonics and phononics encompass the fundamental science of light and sound propagation and interactions in complex structures, as well as its technological applications. This book reviews new and fundamental mathematical tools, computational approaches, and inversion and optimal design methods to address challenging problems in photonics and phononics.
An emphasis is placed on analyzing sub-wavelength resonators, super-focusing and super-resolution of electromagnetic and acoustic waves, photonic and phononic crystals, electromagnetic cloaking, and electromagnetic and elastic metamaterials and metasurfaces. Throughout this book, the authors demonstrate the power of layer potential techniques for solving challenging problems in photonics and phononics when they are combined with asymptotic analysis. This book might be of interest to researchers and graduate students working in the fields of applied and computational mathematics, partial differential equations, electromagnetic theory, elasticity, integral equations, and inverse and optimal design problems in photonics and phononics.

This item will also be of interest to those working in differential equations.

Contents: Introduction; Part 1. Mathematical and computation tools: Generalized argument principle and Rouché's theorem;

Layer potentials; Perturbations of cavities and resonators; Part 2. Diffraction gratings and band-gap materials: Diffraction gratings; Photonic band gaps; Phononic band gaps; Part 3. Sub-wavelength resonant structures and super-resolution: Plasmonic resonances for nanoparticles; Imaging of small particles; Super-resolution imaging; Part 4. Metamaterials: Near-cloaking; Anomalous resonance cloaking and shielding; Plasmonic metasurfaces; Part 5. Sub-wavelength phonics: Helmholtz resonator; Minnaert resonances for bubbles; Appendix A. Spectrum of self-adjoint operators; Appendix B. Optimal control and level set representation; Bibliography; Index.

Mathematical Surveys and Monographs, Volume 235

October 2018, approximately 500 pages, Hardcover, ISBN: $978-$ 1-4704-4800-4, 2010 Mathematics Subject Classification: 47A55, 47A75, 31A10, 34A55, 35R30, 35B34, 45Q05, 30E25, AMS members US\$108, List US\$135, Order code SURV/235

\section{Probability and Statistics}

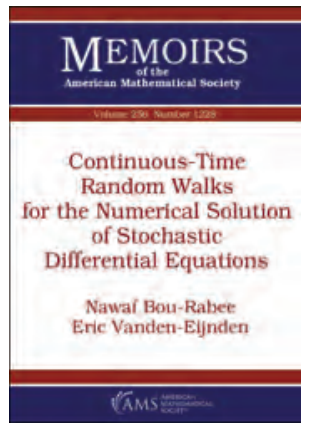

Continuous-Time Random Walks for the Numerical Solution of Stochastic Differential Equations

\author{
Nawaf Bou-Rabee, Rutgers \\ University Camden, NJ, and \\ Eric Vanden-Eijnden, Courant \\ Institute of Mathematical \\ Sciences, New York University, \\ $N Y$
}

Contents: Introduction; Algorithms; Examples and applications; Analysis on gridded state spaces; Analysis on gridless state spaces; Tridiagonal case; Conclusion and outlook; Bibliography.

Memoirs of the American Mathematical Society, Volume 256, Number 1228

October 2018, 124 pages, Softcover, ISBN: 978-1-4704-3181-5, 2010 Mathematics Subject Classification: 65C30; 60J25, 60J75, 
Individual member US\$46.80, List US\$78, Institutional member US\$62.40, Order code MEMO/256/1228

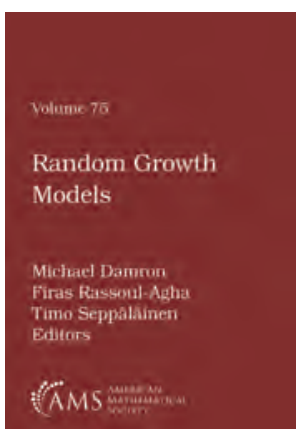

\section{Random Growth Models}

Michael Damron, Georgia

Institute of Technology, Atlanta, $G A$, Firas Rassoul-Agha, University of Utah, Salt Lake City, UT, and Timo Seppäläinen, University of Wisconsin, Madison, WI, Editors

The study of random growth models began in probability theory about 50 years ago, and today this area occupies a central place in the subject. The considerable challenges posed by these models have spurred the development of innovative probability theory and opened up connections with several other parts of mathematics, such as partial differential equations, integrable systems, and combinatorics. These models also have applications to fields such as computer science, biology, and physics.

This volume is based on lectures delivered at the 2017 AMS Short Course "Random Growth Models", held January 2-3, 2017, in Atlanta, GA.

The articles in this book give an introduction to the most-studied models; namely, first- and last-passage percolation, the Eden model of cell growth, and particle systems, focusing on the main research questions and leading up to the celebrated Kardar-Parisi-Zhang equation. Topics covered include asymptotic properties of infection times, limiting shape results, fluctuation bounds, and geometrical properties of geodesics, which are optimal paths for growth.

This item will also be of interest to those working in mathematical physics.

Contents: M. Damron, Random growth models: Shape and convergence rate; J. Hanson, Infinite geodesics, asymptotic directions, and Busemann functions in first-passage percolation; P. Sosoe, Fluctuations in first-passage percolation; F. RassoulAgha, Busemann functions, geodesics, and the competition interface for directed last-passage percolation; T. Seppäläinen, The corner growth model with exponential weights; I. Corwin, Exactly solving the KPZ equation; Index.

Proceedings of Symposia in Applied Mathematics, Volume 75

October 2018, approximately 260 pages, Hardcover, ISBN: 9781-4704-3553-0, 2010 Mathematics Subject Classification: 60K35, 60K37, 82B43, AMS members US\$88, List US\$110, Order code PSAPM/75

\section{New AMS-Distributed Publications}

\section{Algebra and Algebraic Geometry}

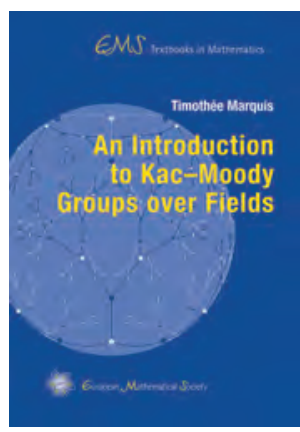

\section{An Introduction to Kac-Moody Groups over Fields}

\author{
Timothée Marquis, Université \\ Catholique de Louvain, \\ Louvain-la-Neuve, Belgium
}

The interest in Kac-Moody algebras and groups has grown exponentially in the past decades, both in the mathematical and physics communities, and with it the need for an introductory textbook on the topic.

The aim of this book is twofold: (1) to offer an accessible, reader-friendly, and self-contained introduction to Kac-Moody algebras and groups; and (2) to "clean the foundations" and provide a unified treatment of the theory.

The book starts with an outline of the classical Lie theory, used to set the scene. Part II provides a self-contained introduction to Kac-Moody algebras. The heart of the book is Part III, which develops an intuitive approach to the construction and fundamental properties of Kac-Moody groups. It is complemented by two appendices that offer introductions to affine group schemes and to the theory of buildings.

A publication of the European Mathematical Society (EMS). Distributed within the Americas by the American Mathematical Society.

EMS Textbooks in Mathematics, Volume 22

August 2018, 343 pages, Hardcover, ISBN: 978-3-03719-187-3, 2010 Mathematics Subject Classification: 20G44, 20E42, 17B67, AMS members US\$46.40, List US\$58, Order code EMSTEXT/22 


\section{Differential Equations}

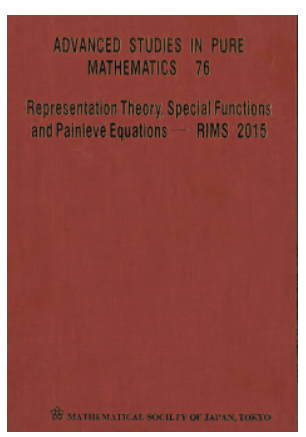

Functions

and Painvlevé
Representation

Theory, Special Equations-RIMS 2015

Hitoshi Konno, Tokyo University of Marine Science and Technology, Japan, Hidetaka Sakai, University of Tokyo, Japan, Junichi Shiraishi, University of Tokyo, Japan, Takao Suzuki, Kindai University, Japan, and Yasuhiko Yamada, Kobe University, Japan, Editors

To celebrate Masatoshi Noumi's 60th birthday, the international conference "Representation Theory, Special Functions and Painlevé Equations" was held at the Research Institute for Mathematical Sciences, Kyoto University, from March 3-March 6, 2015.

This volume is the proceedings of the conference. It contains 15 papers covering a variety of topics in the research fields where Noumi has made many significant contributions over the years-representation theory, special functions, Painlevé equations, among others.

This item will also be of interest to those working in algebra and algebraic geometry.

Published for the Mathematical Society of Japan by Kinokuniya, Tokyo, and distributed worldwide, except in Japan, by the AMS.

Advanced Studies in Pure Mathematics, Volume 76

June 2018, 541 pages, Hardcover, ISBN: 978-4-86497-050-1, 2010 Mathematics Subject Classification: 39-06; 20G42, 20C08, 33-06, 34M55, AMS members US\$77.60, List US\$97, Order code ASPM/76

\section{General Interest}

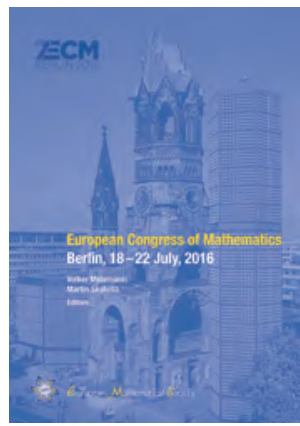

\section{European Congress of} Mathematics

Berlin, July 18-22, 2016

Volker Mehrmann, Technical University of Berlin, Germany, and Martin Skutella, Technical University of Berlin, Germany, Editors

The European Congress of Mathematics, held every four years, is a well-established major international mathematical event. The Seventh European Congress of Mathematics (7ECM) took place in Berlin, Germany, July 18-22, 2016, with about 1100 participants from all over the world.

Ten plenary, thirty-three invited, and four special lectures formed the core of the program. As at all the previous EMS congresses, ten outstanding young mathematicians received the EMS prizes in recognition of their research achievements. In addition, two more prizes were awarded: The Felix Klein Prize for a remarkable solution of an industrial problem, and-for the second time-the Otto Neugebauer Prize for a highly original and influential piece of work in the history of mathematics. The program was complemented by forty-three minisymposia with about 160 talks as well as contributed talks that ranged over all areas of mathematics. Several panel discussions and meetings were organized, covering a variety of issues ranging from the future of mathematical publishing to public awareness of mathematics.

These proceedings present extended versions of most of the plenary and invited lectures delivered during the congress, providing a permanent record of the best of what mathematics offers today.

August 2018, 901 pages, Hardcover, ISBN: 978-3-03719-176-7, 2010 Mathematics Subject Classification: 00Bxx, AMS members US\$110.40, List US\$138, Order code EMSEMC/2016 


\section{Geometry and Topology}

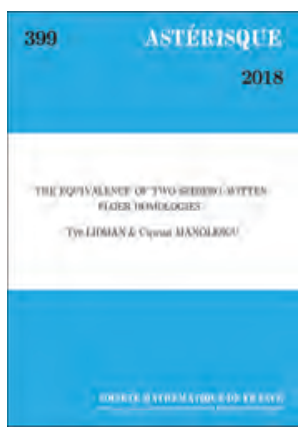

The Equivalence of Two Seiberg-Witten Floer Homologies

Tye Lidman, North Carolina State University, Raleigh, NC, and Ciprian Manolescu, University of California, Los Angeles

The authors show that monopole Floer homology (as defined by Kronheimer and Mrowka) is isomorphic to the $S^{1}$-equivariant homology of the Seiberg-Witten Floer spectrum constructed by Ciprian Manolescu, the co-author of this book.

A publication of the Société Mathématique de France, Marseilles (SMF), distributed by the AMS in the U.S., Canada, and Mexico. Orders from other countries should be sent to the SMF. Members of the SMF receive a $30 \%$ discount from list.

Astérisque, Number 399

June 2018, 220 pages, Softcover, ISBN: 978-2-85629-883-1, 2010 Mathematics Subject Classification: 57R58, AMS members US\$53.60, List US\$67, Order code AST/399

\section{Number Theory}

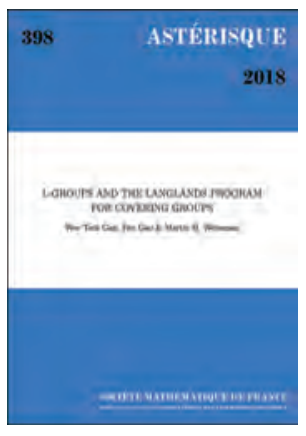

L-Groups and the Langlands Program for Covering Groups

Wee Teck Gan, National University of Singapore, Fan Gao, Purdue University, West Lafayette, IN, and Martin $\mathbf{H}$. Weissman, University of California, Santa Cruz, CA

This volume proposes an extension of the Langlands program to covers of quasisplit groups, where covers are those that arise from central extensions of reductive groups by $K_{2}$. By constructing an $L$-group for any such cover, the authors can conjecture a parameterization of genuine irreducible representations by Langlands parameters. Two constructions of the $L$-group are given and related to each other in a final note.

A publication of the Société Mathématique de France, Marseilles (SMF), distributed by the AMS in the U.S., Canada, and Mexico.

Astérisque, Number 398

June 2018, 286 pages, Softcover, ISBN: 978-2-85629-881-7, 2010 Mathematics Subject Classification: 11F70, 22E50, AMS members US\$60, List US\$75, Order code AST/398

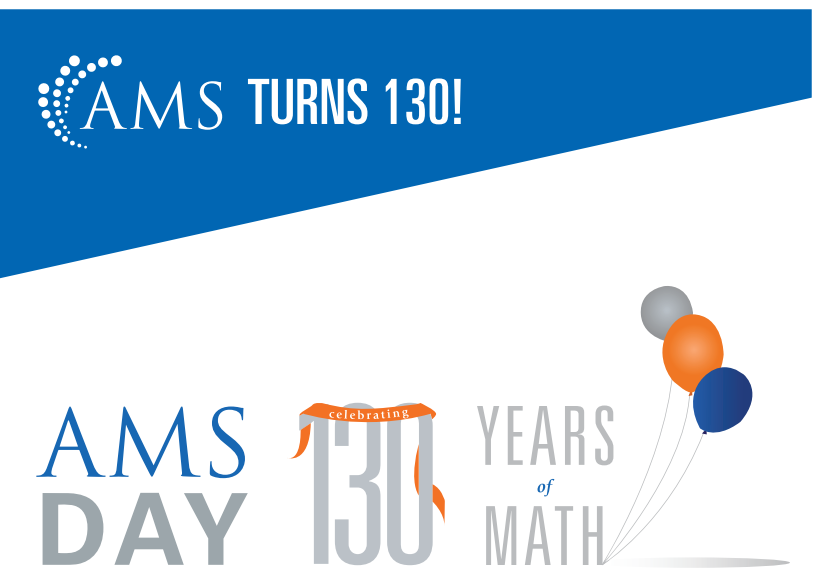

We're celebrating 130 years!

Join us on Monday,

November 26, 2018, as we

honor our AMS members via "AMS Day," a day of specials

on AMS publications, membership, and more! Stay tuned on Facebook, Twitter, and member emails for details about this exciting day. Spread the word about \#AMSDay today!

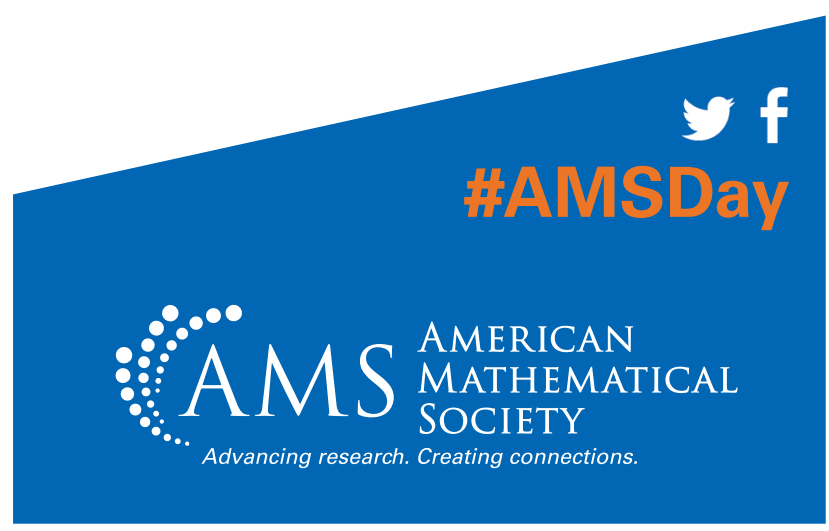




\section{香 港 大 學 \\ THE UNIVERSITY OF HONG KONG}

Founded in 1911, the University of Hong Kong is committed to the highest international standards of excellence in teaching and research, and has been at the international forefront of academic scholarship for many years. The University has a comprehensive range of study programmes and research disciplines spread across 10 faculties and over 140 academic departments and institutes/centres. There are 28,000 undergraduate and postgraduate students who are recruited globally, and more than 2,000 members of academic and academic-related staff coming from multi-cultural backgrounds, many of whom are internationally renowned.

\section{Tenure-track Associate Professor/Assistant Professor in the Research Division of Mathematical and Statistical Science, Faculty of Science (3 posts) (Ref: 201800940)}

The Faculty of Science will be 80 years old in 2019 and to celebrate this anniversary we are recruiting a cohort of 80 th Anniversary professors. This is an unprecedented expansion focused on realizing the Faculty's vision to be pre-eminent in Hong Kong, leading in Asia and highly competitive globally. We are aiming to expand by about $20 \%$ over the next year. The first round of hiring has been very successful and we are recruiting 10 more posts in this second round.

The Faculty of Science is one of the larger faculties in HKU with about 130 academic staff in five departments (Chemistry, Earth Sciences, Mathematics, Physics, and Statistics and Actuarial Science) and one school (Biological Sciences) as well as an off-site marine facility, The Swire Institute of Marine Science (SWIMS). The Faculty provides a supportive and friendly environment and is embarking on a programme of recruitment to invest in areas of acknowledged strength and to invest in areas of internationally competitive activity. Information about the Faculty can be obtained at http://Www.Scifac.hku.hk/. In addition, the Faculty is committed to gender equality and positively welcomes applications from women as well as men. The Faculty will also be happy to consider applications from individuals with their own funding who wish to explore proleptic positions.

To better support research, the Faculty has established a new research division structure and there are six new research divisions:

- Chemistry

- Earth and Planetary Science

- Ecology and Biodiversity
- Mathematical and Statistical Science

- Molecular and Cell Biology

- Physics and Astronomy

The Research Division of Mathematical and Statistical Science covers Pure and Applied Mathematics, Statistics and Actuarial Science. It has strengths in geometry, number theory, optimisation, stochastic analysis and biostatistics with key expertise in complex and algebraic geometry, Lie theory, automorphic form, combinatorial and continuous optimization, actuarial science, time series, survival analysis. We plan to recruit 3-5 new staff in the following areas:

(a) number theory (including representation theory, arithmetic/algebraic geometry)

(b) topological data analysis and related areas (including scientific computing)

(c) big data in environment, society and public health (joint with the Faculty of Business and Economics, please see http://jobs.hku.hk/jd.php?id=201800334)

(d) bioinformatics (joint with Li Ka Shing Faculty of Medicine, please see http://jobs.hku.hk/jd.php?id=201800751) *

(e) actuarial science (focusing on extreme theory, optimal insurance or applied probability)

" These posts have been advertised previously but remain open, see link for application.

Applications are invited for tenure-track appointments as Associate Professor/Assistant Professor in the Research Division of Mathematical and Statistical Science in the Faculty of Science (for the 3 posts in (a), (b) and (e)), to commence on or before September 30, 2019. The posts will initially be made on a three-year fixed-term basis with the possibility of renewal and with consideration for tenure before the expiry of a second three-year appointment

Applicants should possess a Ph.D. degree in Mathematics or Statistical Science. They should have research interests that align with or are complementary to one of the identified areas of recruitment in the Research Division. Appointees will have a lighter teaching load during the first contract. The teaching duties will be assigned by the Head of Department of the relevant department that an appointee will join upon appointment.

Annual salaries will be in the following ranges (subject to review from time to fime at the entire discretion of the University):

$\begin{array}{ll}\text { Associate Professor } \quad: & H K \$ 864,480-1,373,880 \\ \begin{array}{l}\text { Assistant Professor } \\ \text { (approximately US } \$ 1=\text { HK\$7.8) }\end{array} & \text { HK\$683,280-1,018,260 }\end{array}$

A globally competitive remuneration package commensurate with the appointee's qualifications and experience will be offered. At current rates, salaries tax does not exceed $15 \%$ of gross income. The appointment will attract a contract-end gratuity and University contribution to a retirement benefits scheme, totaling up to $15 \%$ of basic salary, as well as leave, and medical benefits. Housing benefits will be provided as applicable.

Applicants should submit a cover letter, a completed application form, together with an up-to-date C.V., which should include hyperlinks to google scholar profiles and ORCID, a detailed publication list since 2014 with journal impact factor and citations indicated for each publication, a research proposal and a teaching statement to:

https://web.science.hku.hk/job/201800940.html (for posts (a), (b) and (e))

Applicants will also be required to arrange 3 reference letters under confidential cover, it is important to note that at least one of the referees should be able to comment, amongst other things, on the applicant's research ability and his/her contribution to teaching or research in his/ her department, while the remaining two should have knowledge of his/her recent academic or employment experience. Referees should submit their reference letters to https://Web.science.hku.hk/job/201800940_ref.html. Application form (341/1111) can be downloaded at http://www.hku.hk/apptunit/form-ext.doc. Enquiries about the various posts should be sent to Head. Department of Mathematics (e-mail: ntw@maths.hku.hk), or Head, Department of Statistics \& Actuarial Science (e-mail: gyin@hku.hk).

Further particulars can be obtained at http://jobs.hku.hk/. Closes November 15, 2018. Interviews are expected to be held from January 7 to $11,2019$.

The University thanks applicants for their interest, but advises that only candidates shortlisted for interviews will be notified of the application result

The University is an equal opportunities employer and

is committed to equality, ethics, inclusivity, diversity and transparency; and is committed to a Non-smoking Policy 


\section{OCTOBER TABLE OF CONTENTS}

The Meetings and Conferences section of the Notices gives information on all AMS meetings and conferences approved by press time for this issue. Please refer to the page numbers cited on this page for more detailed information on each event. Invited Speakers and Special Sessions are listed as soon as they are approved by the cognizant program committee; the codes listed are needed for electronic abstract submission. For some meetings the list may be incomplete. Information in this issue may be dated.
The most up-to-date meeting and conference information can be found online at: www.ams.org/meetings.

Important Information About AMS Meetings: Potential organizers, speakers, and hosts should refer to page 88 in the January 2018 issue of the Notices for general information regarding participation in AMS meetings and conferences.

Abstracts: Speakers should submit abstracts on the easy-to-use interactive Web form. No knowledge of LTEX is necessary to submit an electronic form, although those who use IATEX may submit abstracts with such coding, and all math displays and similarily coded material (such as accent marks in text) must be typeset in LATEX. Visit www.ams.org /cgi-bin/abstracts/abstract.pl. Questions about abstracts may be sent to absinfo@ams.org. Close attention should be paid to specified deadlines in this issue. Unfortunately, late abstracts cannot be accommodated.

\section{MEETINGS IN THIS ISSUE}

\section{8}

September 29-30 Newark, Delaware

p. 1150

October 20-21

Ann Arbor, Michigan

p. 1151

October 27-28

San Francisco, California

p. 1152

November 3-4

Fayetteville, Arkansas

p. 1153

2019

January 16-19 JMM Baltimore, Maryland

March 15-17

March 22-24

Auburn, Alabama

p. 1165

p. 1154

p. 1155

April 13-14

Honolulu, Hawaii

p. 1157

Hartford, Connecticut

p. 1158

June 10-13

Quy Nhon City, Vietnam

p. 1159

p. 1159

October 12-13

Madison, Wisconsin

p. 1159

Gainesville, Florida

p. 1160

November 2-3

Riverside, California

\section{0}

January 15-18

Denver, Colorado

p. 1160

March 13-15

Charlottesville, Virginia p. 1160

May 2-3

Fresno, California

p. 1161

September $12-13$

El Paso, Texas

p. 1161

2021

January 6-9 Washington, DC p. 1161

July 5-9

July 19-23

Grenoble, France

p. 1161

Buenos Aires, Argentina p. 1162

2022

January 5-8 Seattle, Washington

p. 1162

2023

January 4-7 Boston, Massachusetts $\quad$ p. 1162

See www. ams.org/meetings for the most up-to-date information on the meetings and conferences that we offer.

\section{ASSOCIATE SECRETARIES OF THE AMS}

Central Section: Georgia Benkart, University of WisconsinMadison, Department of Mathematics, 480 Lincoln Drive, Madison, WI 53706-1388; email: benkart@math.wisc.edu; telephone: 608-263-4283.

Eastern Section: Steven H. Weintraub, Department of Mathematics, Lehigh University, Bethlehem, PA 18015-3174; email: steve.weintraub@lehigh.edu; telephone: 610-758-3717.
Southeastern Section: Brian D. Boe, Department of Mathematics, University of Georgia, 220 D W Brooks Drive, Athens, GA 30602-7403, email: brian@math.uga.edu; telephone: 706-5422547.

Western Section: Michel L. Lapidus, Department of Mathematics, University of California, Surge Bldg., Riverside, CA 925210135; email: lapidus@math.ucr.edu; telephone:951-827-5910. 


\section{Meetings \& Conferences of the AMS}

IMPORTANT INFORMATION REGARDING MEETINGS PROGRAMS: AMS Sectional Meeting programs do not appear in the print version of the Notices. However, comprehensive and continually updated meeting and program information with links to the abstract for each talk can be found on the AMS website. See www . ams . org/meetings/.

Final programs for Sectional Meetings will be archived on the AMS website accessible from the stated URL.

\section{Newark, Delaware}

\section{University of Delaware}

\section{September 29-30, 2018}

Saturday - Sunday

\section{Meeting \# 1141}

Eastern Section

Associate secretary: Steven H. Weintraub

Announcement issue of Notices: June 2018

Program first available on AMS website: August 9, 2018

Issue of Abstracts: Volume 39, Issue 3

\section{Deadlines}

For organizers: Expired

For abstracts: Expired

The scientific information listed below may be dated. For the latest information, see www. ams.org/amsmtgs /sectional.htm1.

\section{Invited Addresses}

Leslie F Greengard, Courant Institute, NYU and Flatiron Institute, Simons Foundation, Linear and nonlinear inverse problems in imaging.

J. Elisenda Grigsby, Boston College, Braids, surfaces, and homological invariants.

Davesh Maulik, MIT, Enumerative geometry and quantum groups.

\section{Special Sessions}

Advances in Numerical Approximation of Partial Differential Equations, Constantin Bacuta and Jingmei Qiu, University of Delaware.

Applied Algebraic Topology, Chad Giusti, University of Delaware, and Gregory Henselman, Princeton University. Billiard Dynamics: Standard and Alternative Collision Models, Tim Chumley, Mount Holyoke College, Chris Cox,
University of Delaware, and Renato Feres, Washington University in St. Louis. Commutative Algebra, Ela Celikbas, West Virginia University, Sema Gunturkun, University of Michigan, and Oana Veliche, Northeastern University.

Convex Geometry and Functional Inequalities, Mokshay Madiman, University of Delaware, Elisabeth Werner, Case Western Reserve University, and Artem Zvavitch, Kent State University.

Fixed Point Theory with Application and Computation, Clement Boateng Ampadu, Boston, MA, Penumarthy Parvateesam Murthy, Guru Ghasidas Vishwavidyalaya, Bilaspur, India, Naeem Saleem, University of Management and Technology, Lahore, Pakistan, Yaé Ulrich Gaba, Institut de Mathématiques et de Sciences Physiques (IMSP), Porto-Novo, Bénin, and Xavier Udo-utun, University of Uyo, Uyo, Nigeria.

Graph Theory, Sebastian M. Cioabă, University of Delaware, Brian Kronenthal, Kutztown University of Pennsylvania, Felix Lazebnik, University of Delaware, and Wing Hong Tony Wong, Kutztown University of Pennsylvania. Interplay between Analysis and Combinatorics, Mahya Ghandehari and Dominique Guillot, University of Delaware.

Modern Quasiconformal Analysis and Geometric Function Theory, David Herron, University of Cincinnati, and Yuk-J Leung, University of Delaware.

Nonlinear Water Waves and Related Problems, Philippe Guyenne, University of Delaware.

Operator and Function Theory, Kelly Bickel, Bucknell University, Michael Hartz, Washington University, St. Louis, Constanze Liaw, University of Delaware, and Alan Sola, Stockholm University.

Probability, Combinatorics, and Statistical Mechanics, Nayantara Bhatnagar and Douglas Rizzolo, University of Delaware.

Quantum Correlation Sets in Quantum Information Theory, Elie Alhajjar and Travis B. Russell, US Military Academy.

Recent Advances in Nonlinear Schrödinger Equations, Alexander Pankov, Morgan State University, Junping 
Shi, College of William and Mary, and Jun Wang, Jiangsu University.

Recent Analytic and Numeric Results on Nonlinear Evolution Equations, Xiang Xu, Old Dominion University, and Wujun Zhang, Rutgers University.

Representations of Infinite Dimensional Lie Algebras and Applications, Marco Aldi, Virginia Commonwealth University, Michael Penn, Randolph College, and Juan Villarreal, Virginia Commonwealth University.

Stochastic Processes in Mathematical Biology, Yao Li, University of Massachusetts Amherst, and Abhyudai Singh, University of Delaware.

The Mathematics of Swimmers and Active Particles, Louis Rossi, University of Delaware, and Enkeleida Lushi, Flatiron Institute.

\section{Ann Arbor, Michigan University of Michigan, Ann Arbor}

October 20-21, 2018

Saturday - Sunday

\section{Meeting \# 1143}

Central Section

Associate secretary: Georgia Benkart

Announcement issue of Notices: July 2018

Program first available on AMS website: August 30, 2018

Issue of Abstracts: Volume 39, Issue 4

\section{Deadlines}

For organizers: Expired

For abstracts: Expired

The scientific information listed below may be dated. For the latest information, see www. ams.org/amsmtgs /sectional.html.

\section{Invited Addresses}

Elena Fuchs, University of California, Davis, Primes and local to global in circle packings.

Andrew Putman, University of Notre Dame, The mapping class group of a surface.

Charles K Smart, The University of Chicago, The Abelian sandpile and circle packings.

\section{Special Sessions}

Advances in Commutative Algebra, Jack Jeffries, University of Michigan, Linquan Ma, Purdue University, and Karl Schwede, University of Utah.

Advances on Analytical and Geometric Aspects of Differential Equations, Alessandro Arsie, Chunhua Shan, and Ekaterina Shemyakova, University of Toledo.

Analytical and Numerical Aspects of Turbulent Transport, Michele Coti Zelati, Imperial College London, and Ian Tobasco and Karen Zaya, University of Michigan.
Aspects of Geometric Mechanics and Dynamics, Anthony M Bloch and Marta Farre Puiggali, University of Michigan.

Bio-inspired Mechanics and Propulsion, Silas Alben, University of Michigan, and Longhua Zhao, Case Western Reserve University.

Canonical Operators in Several Complex Variables and Related Topics, David Barrett and Luke Edholm, University of Michigan, and Yunus Zeytuncu, University of Michigan, Dearborn.

Cell Motility: Models and Applications, Magdalena Stolarska, University of St. Thomas, and Nicoleta Tarfulea, Purdue University Northwest.

Cluster Algebra, Poisson Geometry, and Related Topics, Eric Bucher, Michigan State University, and Maitreyee Kulkarni and Bach Nguyen, Louisiana State University.

Combinatorics in Algebra and Algebraic Geometry,

Zachary Hamaker, Steven Karp, and Oliver Pechenik, University of Michigan.

Commutative Algebra and Complexity, Harm Derksen, Francesca Gandini, and Visu Makam, University of Michigan.

Commutative Ring Theory, Joe Stickles, Millikin University, and Darrin Weber, University of Evansville.

Ergodic and Topological Quantum Systems, Matthew Cha, Ilya Kachkovskiy, and Shiwen Zhang, Michigan State University.

Extensions-Interpolation-Shape Matching in $R^{d}$, Symmetry-Invariance, Algorithms and Related Topics, Steven Damelin, American Mathematical Society, and Nir Sharon, Princeton University.

From Hyperelliptic to Superelliptic Curves, Tony Shaska, Oakland University, Nicola Tarasca, Rutgers University, and Yuri Zarhin, Pennsylvania State University.

Geometry of Submanifolds, in Honor of Bang-Yen Chens 75th Birthday, Alfonso Carriazo, University of Sevilla, Ivko Dimitric, Penn State Fayette, Yun Myung Oh, Andrews University, Bogdan D. Suceava, California State University, Fullerton, Joeri Van der Veken, University of Leuven, and Luc Vrancken, Universite de Valenciennes.

Interactions between Algebra, Machine Learning and Data Privacy, Jonathan Gryak, University of Michigan, Kelsey Horan, CUNY Graduate Center, Delaram Kahrobaei, CUNY Graduate Center and New York University, Kayvan Najarian and Reza Soroushmehr, University of Michigan, and Alexander Wood, CUNY Graduate Center.

Large Cardinals and Combinatorial Set Theory, Andres E. Caicedo, Mathematical Reviews, and Paul B. Larson, Miami University.

Mathematics of the Genome, Anthony Bloch, Daniel Burns, and Indika Rajapakse, University of Michigan.

Modern Trends in Integrable Systems, Deniz Bilman, Peter Miller, Amber Music, and Guilherme Silva, University of Michigan.

Multiplicities and Volumes: An Interplay Among Algebra, Combinatorics, and Geometry, Federico Castillo, University of Kansas, and Jonathan Montaño, New Mexico State University. 
New Trends in Numerical Methods for Partial Differential Equations: Theory and Applications, Fatih Celiker, Wayne State University.

Nonlocality in Models for Kinetic, Chemical, and Population Dynamics, Christopher Henderson, University of Chicago, Stanley Snelson, Florida Institute of Technology, and Andrei Tarfulea, University of Chicago.

Probabilistic Methods in Combinatorics, Patrick Bennett and Andrzej Dudek, Western Michigan University, and David Galvin, University of Notre Dame.

Random Matrix Theory Beyond Wigner and Wishart, Elizabeth Meckes and Mark Meckes, Case Western Reserve University, and Mark Rudelson, University of Michigan.

Recent Advances in Nonlinear PDE, Jessica Lin, McGill University, and Russell Schwab, Michigan State University.

Recent Developments in Discontinuous Galerkin Methods for Differential Equations, Mahboub Baccouch, University of Nebraska at Omaha.

Recent Developments in Mathematical Analysis of Some Nonlinear Partial Differential Equations, Mimi Dai, University of Illinois at Chicago.

Recent Developments in the Mathematics of Tomography and Scattering, Shixu Meng, University of Michigan, and Yang Yang, Michigan State University.

Recent Trends on Local, Nonlocal and Fractional Partial Differential Equations, Pablo Raúl Stinga, Iowa State University, Peiyong Wang, Wayne State University, and Jiuyi Zhu, Louisiana State University.

Representations of Reductive Groups over Local Fields and Related Topics, Anne-Marie Aubert, Institut Mathématiques de Jussieu, Paris Rive Gauche, Jessica Fintzen, IAS, University of Michigan, University of Cambridge, and Camelia Karimianpour, University of Michigan.

Self-similarity and Long-range Dependence in Stochastic Processes, Takashi Owada, Purdue University, Yi Shen, University of Waterloo, and Yizao Wang, University of Cincinnati.

Structured Homotopy Theory, Thomas Fiore, University of Michigan, Dearborn, Po Hu and Dan Isaksen, Wayne State University, and Igor Kriz, University of Michigan.

The Mathematics of Decisions, Elections, and Games, Michael A. Jones, Mathematical Reviews, and David McCune, William Jewell College.

Topics in Graph Theory, Hypergraphs and Set Systems, John Engbers, Marquette University, and Cliff Smyth, University of North Carolina, Greensboro.

\section{San Francisco, California}

\section{San Francisco State University}

October 27-28, 2018

Saturday - Sunday

\section{Meeting \# 1144}

Western Section

Associate secretary: Michel L. Lapidus

Announcement issue of Notices: July 2018

Program first available on AMS website: September 6, 2018 Issue of Abstracts: Volume 39, Issue 4

\section{Deadlines}

For organizers: Expired

For abstracts: Expired

The scientific information listed below may be dated. For the latest information, see www. ams.org/amsmtgs /sectional.html.

\section{Invited Addresses}

Srikanth B Iyengar, University of Utah, Finite free complexes over polynomial rings.

Sarah Witherspoon, Texas A\&M University, Derivatives, derivations, and Hochschild cohomology.

Abdul-Aziz Yakubu, Howard University, Population cycles in discrete-time infectious disease models.

\section{Special Sessions}

Advances in Operator Theory, Operator Algebras, and Operator Semigroups, Asuman G. Aksoy, Claremont McKenna College, Michael Hartglass, Santa Clara University, Zair Ibragimov, California State University, Fullerton, and Marat Markin, California State University, Fresno.

Algebraic Geometry, Emily Clader and Dustin Ross, San Francisco State University, and Mark Shoemaker, Colorado State University.

Analysis and Geometry of Fractals, Kyle Hambrook, University of Rochester, Chun-Kit Lai, San Francisco State University, and Sze-Man Ngai, Georgia Southern University.

Applied Harmonic Analysis: Frame Theory and Applications, Chun-Kit Lai and Shidong Li, San Francisco State University.

Big Data and Statistical Analytics, Tao He, Mohammad Kafai, and Alexandra Piryatinska, San Francisco State University.

Combinatorial and Categorical Aspects of Representation Theory, Nicholas Davidson and Jonathan Kujawa, University of Oklahoma, and Robert Muth, Tarleton State University. 
Coupling in Probability and Related Fields, Sayan Banerjee, University of North Carolina, Chapel Hill, and Terry Soo, University of Kansas.

Geometric Analysis, Ovidiu Munteanu, University of Connecticut, and David Bao, San Francisco State University.

Geometric Methods in Hypercomplex Analysis, Paula Cerejeiras, Universidade de Aveiro, Matvei Libine, Indiana University, Bloomington, and Mihaela B. Vajiac, Chapman University.

Geometric and Analytic Inequalities and their Applications, Nicholas Brubaker, Isabel M. Serrano, and Bogdan D. Suceavă, California State University, Fullerton.

Homological Aspects in Commutative Algebra and Representation Theory, Srikanth B. Iyengar, University of Utah, and Julia Pevtsova, University of Washington.

Homological Aspects of Noncommutative Algebra and Geometry, Dan Rogalski, University of California San Diego, Sarah Witherspoon, Texas A\&M University, and James Zhang, University of Washington, Seattle.

Markov Processes, Gaussian Processes and Applications, Alan Krinik and Randall J. Swift, California State Polytechnic University.

Mathematical Biology with a focus on Modeling, Analysis, and Simulation, Jim Cushing, The University of Arizona, Saber Elaydi, Trinity University, Suzanne Sindi, University of California, Merced, and Abdul-Aziz Yakubu, Howard University.

Mathematical Methods for the study of the Three Dimensional Structure of Biopolymers, Javier Arsuaga and Mariel Vazquez, University of California Davis, Davis, and Robin Wilson, Cal Poly Pomona.

Noncommutative Geometry and Fundamental Applications, Konrad Aguilar, Arizona State University, and Frederic Latremoliere, University of Denver.

Nonlocal PDEs via Harmonic Analysis, Tadele Mengesha, University of Tennessee, Knoxville, and Armin Schikorra, University of Pittsburgh.

Probabilistic and Statistical Problems in Stochastic Dynamics, Tao He, Mohammad Kafai, and Alexandra Piryatinska, San Francisco State University.

Research in Mathematics by Early Career Graduate Students, Michael Bishop, Marat Markin, Jenna Tague, and Khang Tran, California State University, Fresno.

Social Change In and Through Mathematics and Education, Federico Ardila and Matthias Beck, San Francisco State University, Jamylle Carter, Diablo Valley Community College, and Kimberly Seashore, San Francisco State University.

Statistical and Geometrical Properties of Dynamical Systems, Joanna Furno and Matthew Nicol, University of Houston, and Mariusz Urbanski, University of North Texas.

Topics in Operator Theory: CANCELLED, Anna Skripka and Maxim Zinchenko, University of New Mexico.

\section{Fayetteville, Arkansas}

University of Arkansas

November 3-4, 2018

Saturday - Sunday

\section{Meeting \# 1142}

Southeastern Section

Associate secretary: Brian D. Boe

Announcement issue of Notices: July 2018

Program first available on AMS website: August 16, 2018

Issue of Abstracts: Volume 39, Issue 4

\section{Deadlines}

For organizers: Expired

For abstracts: Expired

The scientific information listed below may be dated. For the latest information, see www.ams.org/amsmtgs /sectiona1.htm1.

\section{Invited Addresses}

Mihalis Dafermos, Princeton University, On falling into black holes.

Jonathan Hauenstein, University of Notre Dame, $\mathrm{Nu}$ merical algebraic geometry and optimization.

Kathryn Mann, Brown University, Group actions, geometry and rigidity.

\section{Special Sessions}

Advances in Birational Geometry, Roi Docampo, University of Oklahoma, and Lance Edward Miller and Wenbo Niu, University of Arkansas.

Commutative Algebra, Alessandro De Stefani, University of Nebraska-Lincoln, Paolo Mantero, University of Arkansas, and Thomas Polstra, University of Utah.

Groups in Low-dimensional Topology and Dynamics, Matt Clay, University of Arkansas, and Kathryn Mann, Brown University.

Harmonic Analysis and Partial Differential Equations, Ariel Barton, University of Arkansas, and Simon Bortz, University of Minnesota.

Interactions Between Combinatorics and Commutative Algebra, Ashwini Bhat, Chris Francisco, and Jeffrey Mermin, Oklahoma State University.

Interactions Between Contact and Symplectic Geometry and Low-dimensional Topology, Jeremy Van Horn-Morris, University of Arkansas, and David Shea Vela-Vick, Louisiana State University.

Non-associative Algebraic Structures and their (Co)homology Theories, Michael Kinyon, University of Denver, Jozef H Przytycki, The George Washington University, and Petr Vojtechovsky and Seung Yeop Yang, University of Denver. 
Numerical Methods for Nonlinear Systems, Jonathan Hauenstein and Tingting Tang, University of Notre Dame.

Operator Theory and Function Spaces of Analytic Functions, Daniel Luecking and Maria Tjani, University of Arkansas.

Partial Differential Equations in Several Complex Variables, Phillip Harrington and Andrew Raich, University of Arkansas.

Recent Advances in Mathematical Fluid Mechanics, Zachary Bradshaw, University of Arkansas.

Recent Developments on Fluid Turbulence, Eleftherios

Gkioulekas, University of Texas Rio Grande Valley.

The Geometry of Curves and Applications, Jason Cantarella and Philipp Reiter, University of Georgia.

Validation and Verification Strategies in Multiphysics Problems, Tulin Kaman, University of Arkansas.

\section{JMM 2019- \\ Baltimore, Maryland}

Baltimore Convention Center,

Hilton Baltimore, and

Baltimore Marriott Inner Harbor Hotel

January 16-19, 2019

Wednesday - Saturday

See page 1165 for full meeting listing and travel information.

\section{Auburn, Alabama}

\section{Auburn University}

March 15-17,2019

Friday - Sunday

\section{Meeting \# 1146}

Southeastern Section

Associate secretary: Brian D. Boe

Announcement issue of Notices: January 2019

Program first available on AMS website: January 31, 2019

Issue of Abstracts: Volume 40, Issue 2

\section{Deadlines}

For organizers: Expired

For abstracts: January 22, 2019

The scientific information listed below may be dated. For the latest information, see www. ams.org/amsmtgs /sectional.html.

\section{Invited Addresses}

Grigoriy Blekherman, Georgia Institute of Technology, To be announced.
Carina Curto, Pennsylvania State University, To be announced.

Ming Liao, Auburn University, To be announced.

\section{Special Sessions}

If you are volunteering to speak in a Special Session, you should send your abstract as early as possible via the abstract submission form found at www. ams.org/cgi-bin /abstracts/abstract.p1.

Algebraic and Discrete Methods in Mathematical Biology (Code: SS 21A), Carina Curto, The Pennsylvania State University, Katherine Morrison, University of Northern Colorado, and Nora Youngs, Colby College.

Applications of Algebraic Geometry (Code: SS 25A), Greg Blekherman, Georgia Institute of Technology, Michael Burr, Clemson University, and Tianran Chen, Auburn University at Montgomery.

Clustering Methods and Applications (Code: SS 23A), Benjamin McLaughlin, Naval Surface Warfare Center Panama City Division (NSWCPCD), and Sung Ha Kang, Georgia Institute of Technology.

Combinatorial Matrix Theory (Code: SS 2A), Zhongshan Li, Georgia State University, and Xavier Martínez-Rivera, Auburn University.

Commutative and Combinatorial Algebra (Code: SS 3A), Selvi Kara Beyarslan, University of South Alabama, and Alessandra Costantini, Purdue University.

Developments in Commutative Algebra (Code: SS 1A), Eloísa Grifo, University of Michigan, and Patricia Klein, University of Kentucky.

Differential Equations in Mathematical Biology (Code: SS 7A), Guihong Fan, Columbus State University, Zhongwei Shen, University of Alberta, and Xiaoxia Xie, Idaho State University.

Discrete and Convex Geometry (Code: SS 17A), Andras Bezdek, Auburn University, Ferenc Fodor, University of Szeged, and Wlodzimierz Kuperberg, Auburn University.

Evolution Equations and Applications (Code: SS 9A), Dmitry Glotov, Wenxian Shen, and Paul G. Schmidt, Auburn University.

Experimental Mathematics in Number Theory, Analysis, and Combinatorics (Code: SS 6A), Amita Malik, Rutgers University, and Armin Straub, University of South Alabama.

Geometric Flows and Minimal Surfaces (Code: SS 20A), Theodora Bourni, University of Tennessee, and Giuseppe Tinaglia, King's College London and University of Tennessee.

Geometric Methods in Representation Theory (Code: SS 15A), Jiuzu Hong and Shrawan Kumar, University of North Carolina, Chapel Hill, and Yiqiang Li, University at Buffalo, the State University of New York.

Geometric and Combinatorial Aspects of Representation Theory (Code: SS 19A), Mark Colarusso, University of South, and Jonas Hartwig, Iowa State University.

Geometry and Topology of Low Dimensional Manifolds, and Their Invariants (Code: SS 13A), John Etnyre, Geor- 
gia Institute of Technology, Bulent Tosun, University of Alabama, and Shea Vela-Vick, Louisiana State University. Graph Theory in Honor of Robert E. Jamison's 70th Birthday (Code: SS 4A), Robert A Beeler, East Tennessee State University, Gretchen Matthews, Virginia Tech, and Beth Novick, Clemson University.

Hopf Algebras and Their Applications (Code: SS 10A), Robert Underwood, Auburn University at Montgomery, and Alan Koch, Agnes Scott College.

Mapping Class Groups (Code: SS 27A), Joan Birman, Columbia University, and Kevin Kordek and Dan Margalit, Georgia Institute of Technology.

Mathematical Analysis and Control Theory of Coupled Partial Differential Equation Models (Code: SS 11A), George Avalos and Pelin Gu"ven Geredeli, University of Nebraska-Lincoln, and László Kindrat, University of New Hampshire.

Nonlinear Reaction-Diffusion Equations and Their Applications (Code: SS 18A), Jerome Goddard,II, Auburn University at Montgomery, Nsoki Mavinga, Swarthmore College, Quinn Morris, Appalachian State University, and R. Shivaji, University of North Carolina at Greensboro. Probability and Stochastic Processes (Code: SS 5A), Ming

Liao, Erkan Nane, and Jerzy Szulga, Auburn University. Random Discrete Structures (Code: SS 24A), Lutz P Warnke, Georgia Institute of Technology, and Xavier Pérez-Giménez, University of Nebraska-Lincoln.

Recent Advances in Coarse Geometry (Code: SS 12A), Jerzy Dydak, University of Tennessee.

Recent Advances in Numerical Methods for PDEs and PDE-constrained Optimization (Code: SS 26A), Yanzhao Cao, Thi-Thao-Phuong Hoang, and Junshan Lin, Auburn University.

Recent Developments in Graph Theory (Code: SS 16A), Xiaofeng Gu, Jeong-Hyun Kang, David Leach, and Rui $\mathrm{Xu}$, University of West Georgia.

Representations of Lie Algebras, Algebraic Groups, and Quantum Groups (Code: SS 8A), Joerg Feldvoss, University of South Alabama, Lauren Grimley, Spring Hill College, and Cornelius Pillen, University of South Alabama.

The Modeling and Analysis of Spatially Extended Structures (Code: SS 22A), Shibin Dai, University of Alabama, Keith Promislow, Michigan State University, and Qiliang Wu, Ohio University.

Topological Data Analysis, Statistics and Applications (Code: SS 14A), Yu-Min Chung, University of North Carolina at Greensboro, and Vasileios Maroulas, University of Tennessee.

\section{Honolulu, Hawaii}

\section{University of Hawaii at Manoa}

\section{March 22-24, 2019}

Friday - Sunday

\section{Meeting \# 1147}

Central Section

Associate secretaries: Georgia Benkart and Michel L. Lapidus

Announcement issue of Notices: January 2019

Program first available on AMS website: February 7, 2019

Issue of Abstracts: Volume 40, Issue 2

\section{Deadlines}

For organizers: Expired

For abstracts: January 29, 2019

The scientific information listed below may be dated. For the latest information, see www. ams.org/amsmtgs /sectiona1.htm1.

\section{Invited Addresses}

Barry Mazur, Harvard University, On the arithmetic of curves (Einstein Public Lecture in Mathematics).

Aaron Naber, Northwestern University, Analysis of geometric nonlinear partial differential equations.

Deanna Needell, University of California, Los Angeles, Simple approaches to complicated data analysis.

Katherine Stange, University of Colorado, Boulder, Title to be announced.

Andrew Suk, University of California, San Diego, Title to be announced.

\section{Special Sessions}

If you are volunteering to speak in a Special Session, you should send your abstract as early as possible via the abstract submission form found at www. ams.org/cgi-bin /abstracts/abstract.p1.

Advances in Iwasawa Theory (Code: SS 12A), Frauke Bleher, University of Iowa, Ted Chinburg, University of Pennsylvania, and Robert Harron, University of Hawaii at Manoa.

Advances in Mathematical Fluid Mechanics (Code: SS 32A), Kazuo Yamazaki, University of Rochester, and Adam Larios, University of Nebraska - Lincoln.

Algebraic Groups, Galois Cohomology, and Local-Global Principles (Code: SS 3A), Raman Parimala, Emory University, Andrei Rapinchuk, University of Virginia, and Igor Rapinchuk, Michigan State University.

Algebraic Number Theory and Diophantine Equations (Code: SS 20A), Claude Levesque, University of Laval.

Algebraic Points (Code: SS 36A), Barry Mazur and Hector Pasten, Harvard University.

Algebraic and Combinatorial Structures in Knot Theory (Code: SS 9A), Sam Nelson, Claremont McKenna College, 
Natsumi Oyamaguchi, Shumei University, and Kanako Oshiro, Sophia University.

Algebraic and Geometric Combinatorics (Code: SS 17A), Andrew Berget, Western Washington University, and Steven Klee, Seattle University.

Analysis of Nonlinear Geometric Equations (Code: SS 23A), Aaron Naber, Northwestern University, and Richard Bamler, University of California Berkeley.

Analytic and Probabilistic Methods in Convex Geometry (Code: SS 27A), Alexander Koldobsky, University of Missouri, Alexander Litvak, University of Alberta, Dmitry Ryabogin, Kent State University, Vladyslav Yaskin, University of Alberta, and Artem Zvavitch, Kent State University.

Applications of Ultrafilters and Nonstandard Methods (Code: SS 33A), Isaac Goldbring, University of California, Irvine, and Steven Leth, University of Northern Colorado.

Arithmetic Dynamics (Code: SS 29A), Andrew Bridy, Texas A\&M University, Michelle Manes, University of Hawai'i at Manoa, and Bianca Thompson, Harvey Mudd College.

Arithmetic Geometry and Its Connections (Code: SS 51A), Laura Capuano, University of Oxford, and Amos Turchet, University of Washington.

Arithmetic and Transcendence of Special Functions and Special Values (Code: SS 56A), Matthew A. Papanikolas, Texas A\&M University, and Federico Pellarin, Université Jean Monnet, St. Étienne.

Coarse Geometry, Index Theory, and Operator Algebras: Around the Mathematics of John Roe (Code: SS 53A), Erik Guentner, University of Hawai'i at Manoa, Nigel Higson, Penn State University, and Rufus Willett, University of Hawai'i at Manoa.

Coding Theory and Information Theory (Code: SS 39A), Manabu Hagiwara, Chiba University, and James B. Nation, University of Hawaii.

Combinatorial and Experimental Methods in Mathematical Phylogeny (Code: SS 47A), Sean Cleary, City College of New York and the CUNY Graduate Center, and Katherine St. John, Hunter College and the American Museum of Natural History.

Commutative Algebra and its Environs (Code: SS 4A), Olgur Celikbas and Ela Celikbas, West Virginia University, and Ryo Takahashi, Nagoya University.

Computability, Complexity, and Learning (Code: SS 45A), Achilles A. Beros and Bj\orn Kjos-Hanssen, University of Hawai'i at Manoa.

Computational and Data-Enabled Sciences (Code: SS 54A), Roummel Marcia, Boaz Ilan, and Suzanne Sindi, University of California, Merced.

Constructive Aspects of Complex Analysis (Code: SS 7A), Ilia Binder and Michael Yampolsky, University of Toronto, and Malik Younsi, University of Hawaii at Manoa.

Differential Geometry (Code: SS 10A), Vincent B. Bonini, Cal Poly San Luis Obispo, Jie Qing, University of California, Santa Cruz, and Bogdan D. Suceava, California State University, Fullerton.

Dynamical Systems and Algebraic Combinatorics (Code: SS 57A), Maxim Arnold, University of Texas at Dallas, Jes- sica Striker, North Dakota State University, and Nathan Williams, University of Texas at Dallas.

Emerging Connections with Number Theory (Code: SS 43A), Katherine Stange, University of Colorado, Boulder, and Renate Scheidler, University of Calgary.

Equivariant Homotopy Theory and Trace Methods (Code: SS 58A), Andrew Blumberg, University of Texas, Teena Gerhardt, Michigan State University, Michael Hill, UCLA, and Michael Mandell, Indiana University.

Factorization and Arithmetic Properties of Integral Domains and Monoids (Code: SS 31A), Scott Chapman, Sam Houston State University, Jim Coykendall, Clemson University, and Christopher O’Neill, University California, Davis.

Generalizations of Symmetric Spaces (Code: SS 22A), Aloysius Helminck, University of Hawaii at Manoa, Vicky Klima, Appalachian State University, Jennifer Schaefer, Dickinson College, and Carmen Wright, Jackson State University.

Geometric Approaches to Mechanics and Control (Code: SS 55A), Monique Chyba, University of Hawaii at Manoa, Tomoki Ohsawa, The University of Texas at Dallas, and Vakhtang Putkaradze, University of Alberta.

Geometry, Analysis, Dynamics and Mathematical Physics on Fractal Spaces (Code: SS 8A), Joe P. Chen, Colgate University, Lũ (Tim) Hùng, Hawai'i Pacific University, Machiel van Frankenhuijsen, Utah Valley University, and Robert G. Niemeyer, University of the Incarnate Word.

Homotopy Theory (Code: SS 48A), Kyle Ormsby and Angélica Osorno, Reed College.

Interactions between Geometric Measure Theory, PDE, and Harmonic Analysis (Code: SS 41A), Mark Allen, Brigham Young University, Spencer Becker-Kahn, University of Washington, Max Engelstein, Massachusetts Institute of Technology, and Mariana Smit Vega Garcia, University of Washington.

Interactions between Noncommutative Algebra and Noncommutative Algebraic Geometry (Code: SS 24A), Garrett Johnson, North Carolina Central University, Bach Nguyen and Xingting Wang, Temple University, and Daniel Yee, Bradley University.

Lie Theory in the Representations of Groups and Related Structures (Code: SS 14A), Christopher Drupieski, DePaul University, and Kay Magaard, University of Arizona.

Mapping Class Groups (Code: SS 35A), Asaf Hadari, University of Hawaii.

Mathematical Analysis of Nonlinear Phenomena (Code: SS 16A), Mimi Dai, University of Illinois at Chicago.

Mathematical Methods and Models in Medicine (Code: SS 19A), Monique Chyba, University of Hawaii, and Jakob Kotas, University of Hawaii and University of Portland.

New Trends in Geometric Measure Theory (Code: SS 37A), Antonio De Rosa, Courant Institute of Mathematical Sciences, New York University, and Luca Spolaor, Massachusetts Institute of Technology.

New Trends on Variational Calculus and Non-Linear Partial Differential Equations (Code: SS 44A), Craig Cowan, University of Manitoba, Michinori Ishiwata, Osaka Univer- 
sity, Abbas Moameni, Carleton University, and Futoshi Takahashi, Osaka City University.

Nonlinear Wave Equations and Applications (Code: SS 42A), Boaz Ilan, University of California, Merced, and Barbara Prinari, University of Colorado, Colorado Springs. Numerical Methods for Partial Differential Equations (Code: SS 50A), Evan Gawlik, Michael Holst, and Martin Licht, University of California, San Diego.

Real and Complex Singularities (Code: SS 34A), Les-

lie Charles Wilson, University of Hawaii, Manoa, Goo Ishikawa, Hokkaido University, and David Trotman, Université de Provence.

Recent Advances and Applications of Modular Forms (Code: SS 1A), Amanda Folsom, Amherst College, Pavel Guerzhoy, University of Hawaii at Manoa, Masanobu Kaneko, Kyushu University, and Ken Ono, Emory University.

Recent Advances in Lie and Related Algebras and their Representations (Code: SS 28A), Brian D. Boe, University of Georgia, and Jonathan Kujawa, University of Oklahoma.

Recent Advances in Numerical Methods for PDEs (Code: 2249A), Hengguang Li, Wayne State University, and Sara Pollock, University of Florida.

Recent Advances in Numerical Methods for PDEs (Code: SS 49A), Hengguang Li, Wayne State University, and Sara Pollock, University of Florida.

Recent Developments in Automorphic Forms (Code: SS 2A), Solomon Friedberg, Boston College, and Jayce Getz, Duke University.

Recent Trends in Algebraic Graph Theory (Code: SS 26A), Sebastian Cioaba, University of Delaware, and Shaun Fallat, University of Regina.

SYZ Mirror Symmetry and Enumerative Geometry (Code: SS 11A), Siu Cheong Lau, Boston University, Naichung Leung, The Chinese University of Hong Kong, and Hsian-Hua Tseng, Ohio State University.

Several Complex Variables (Code: SS 5A), Peter Ebenfelt, University of California, San Diego, John Erik Fornaess, University of Michigan and Norwegian University of Science and Technology, Ming Xiao, University of California, San Diego, and Yuan Yuan, Syracuse University.

Spaces of Holomorphic Functions and Their Operators (Code: SS 21A), Mirjana Jovovic and Wayne Smith, University of Hawaii.

Sparsity, Randomness, and Optimization (Code: SS 15A), Deanna Needell and Jamie Haddock, University of California, Los Angeles.

Spectral Geometry: The Length and Laplace Spectra of Riemannian Manifolds (Code: SS 25A), Benjamin Linowitz, Oberlin College, and Jeffrey S. Meyer, California State University at San Bernardino.

Stability and Singularity in Fluid Dynamics (Code: SS 40A), Tristan Buckmaster, Princeton University, Steve Shkoller, University of California, Davis, and Vlad Vicol, Princeton University.

Structural Graph Theory (Code: SS 30A), Zixia Song, University of Central Florida, Martin Rolek, College of William and Mary, and Yue Zhao, University of Central Florida.
The Mathematics of Cryptography (Code: SS 18A), Shahed Sharif, California State University, San Marcos, and Alice Silverberg, University of California, Irvine.

Three-dimensional Floer Theory, Contact Geometry, and Foliations (Code: SS 6A), Joan Licata, Australian National University, and Robert Lipshitz, University of Oregon.

Topics at the Interface of Analysis and Geometry (Code: SS 38A), Alex Austin and Sylvester Eriksson-Bique, University of California, Los Angeles.

Valuations on Algebraic Function Fields and Their Subrings (Code: SS 46A), Ron Brown, University of Hawaii, Steven Dale Cutkosky, University of Missouri, and FranzViktor Kuhlmann, University of Szczecin.

What is Happening in Mathematical Epidemiology? Current Theory, New Methods, and Open Questions (Code: SS 52A), Olivia Prosper, University of Kentucky.

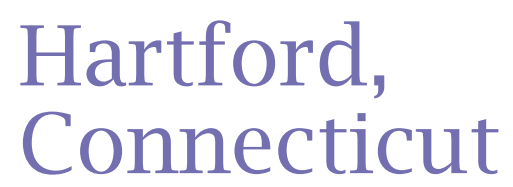

University of Connecticut Hartford (Hartford Regional Campus)

April 13-14, 2019

Saturday - Sunday

\section{Meeting \# 1148}

Eastern Section

Associate secretary: Steven H. Weintraub

Announcement issue of Notices: February 2019

Program first available on AMS website: February 21, 2019

Issue of Abstracts: Volume 40, Issue 2

\section{Deadlines}

For organizers: Expired

For abstracts: February 5, 2019

The scientific information listed below may be dated. For the latest information, see www.ams.org/amsmtgs /sectional.html.

\section{Invited Addresses}

Olivier Bernardi, Brandeis University, Title to be announced.

Brian Hall, Notre Dame University, Title to be announced.

Christina Sormani, City University of New York, Title to be announced.

\section{Special Sessions}

If you are volunteering to speak in a Special Session, you should send your abstract as early as possible via the abstract submission form found at www. ams.org/cgi-bin /abstracts/abstract.p1. 


\section{MEETINGS \& CONFERENCES}

Analysis, Geometry, and PDEs in Non-smooth Metric Spaces (Code: SS 1A), Vyron Vellis, University of Connecticut, Xiaodan Zhou, Worcester Polytechnic Institute, and Scott Zimmerman, University of Connecticut.

Banach Space Theory and Metric Embeddings (Code: SS 11A), Mikhail Ostrovskii, St. John's University, and Beata Randrianantoanina, Miami University.

Cluster Algebras and Related Topics (Code: SS 12A), Emily Gunawan and Ralf Schiffler, University of Connecticut.

Computability Theory (Code: SS 2A), Damir Dzhafarov and Reed Solomon, University of Connecticut, and Linda Brown Westrick, Pennsylvania State University.

Mathematical Cryptology (Code: SS 8A), Lubjana Beshaj, United States Military Academy, and Jaime Gutierrez, University of Cantabria, Santander, Spain.

Modeling and Qualitative Study of PDEs from Materials Science and Geometry. (Code: SS 6A), Yung-Sze Choi, Changfeng Gui, and Xiaodong Yan, University of Connecticut.

Recent Development of Geometric Analysis and Nonlinear PDEs (Code: SS 3A), Ovidiu Munteanu, Lihan Wang, and Ling Xiao, University of Connecticut.

Representation Theory of Quantum Algebras and Related Topics (Code: SS 10A), Drew Jaramillo, University of Connecticut, Garrett Johnson, North Carolina Central University, and Margaret Rahmoeller, Roanoke College.

Special Session on Regularity Theory of PDEs and Calculus of Variations on Domains with Rough Boundaries (Code: SS 5A), Murat Akman, University of Connecticut, and Zihui Zhao, University of Washington.

Stochastic Analysis and Related Fields (Code: SS 7A), Fabrice Baudoin, University of Connecticut, and Cheng Ouyang, University of Illinois at Chicago.

Stochastic Processes, Random Walks, and Heat Kernels (Code: SS 4A), Patricia Alonso Ruiz, University of Connecticut, and Phanuel Mariano, Purdue University.

Sub-Riemannian and CR Geometric Analysis (Code: SS 9A), Fabrice Baudoin, University of Connecticut, and Luca Capogna, Worcester Polytechnic Institute.

\section{Quy Nhon City, Vietnam}

\section{Quy Nhon University}

June 10-13, 2019

Monday - Thursday

\section{Meeting \# 149}

Associate secretary: Brian D. Boe

Announcement issue of Notices: April 2019

Program first available on AMS website: To be announced Issue of Abstracts: To be announced

\section{Deadlines}

For organizers: November 30, 2018

For abstracts: To be announced

The scientific information listed below may be dated. For the latest information, see www.ams.org/amsmtgs /internmtgs.html.

\section{Invited Addresses}

Henry Cohn, Microsoft Research, To be announced.

Robert Guralnick, University of Southern California, To be announced.

Le Tuan Hoa, Hanoi Institute of Mathematics, To be announced.

Nguyen Dong Yen, Hanoi Institute of Mathematics, To be announced.

Zhiwei Yun, Massachusetts Institute of Technology, To be announced.

Nguyen Tien Zung, Toulouse Mathematics Institute, To be announced. 


\section{Madison, Wisconsin}

\section{University of Wisconsin-Madison}

\section{September 14-15, 2019}

Saturday - Sunday

\section{Meeting \# 1150}

Central Section

Associate secretary: Georgia Benkart

Announcement issue of Notices: June 2019

Program first available on AMS website: July 23, 2019

Issue of Abstracts: Volume 40, Issue 3

\section{Deadlines}

For organizers: February 14, 2019

For abstracts: July 16, 2019

The scientific information listed below may be dated. For the latest information, see www. ams.org/amsmtgs /sectional.html.

\section{Invited Addresses}

Nathan Dunfield, University of Illinois, Urbana-Champaign, Title to be announced.

Teena Gerhardt, Michigan State University, Title to be announced.

Lauren Williams, University of California, Berkeley, Title to be announced (Erdős Memorial Lecture).

\section{Special Sessions}

If you are volunteering to speak in a Special Session, you should send your abstract as early as possible via the abstract submission form found at www. ams. org/cgi-bin /abstracts/abstract.p1.

Homological and Characteristic $p>0$ Methods in Commutative Algebra (Code: SS 1A), Michael Brown, University of Wisconsin-Madison, and Eric Canton, University of Michigan.

Recent Developments in Harmonic Analysis (Code: SS 3A), Theresa Anderson, Purdue University, and Joris Roos, University of Wisconsin-Madison.

Recent Work in the Philosophy of Mathematics (Code: SS 4A), Thomas Drucker, University of Wisconsin-Whitewater, and Dan Sloughter, Furman University.

Special Functions and Orthogonal Polynomials (Code: SS 2A), Sarah Post, University of Hawai'i at Manoa, and Paul Terwilliger, University of Wisconsin-Madison.

\section{Binghamton, New York}

\section{Binghamton University}

October 12-13,2019

Saturday - Sunday

\section{Meeting \#1151}

Eastern Section

Associate secretary: Steven H. Weintraub

Announcement issue of Notices: August 2019

Program first available on AMS website: August 29, 2019

Issue of Abstracts: Volume 40, Issue 3

\section{Deadlines}

For organizers: March 12, 2019

For abstracts: August 20, 2019

The scientific information listed below may be dated. For the latest information, see www.ams.org/amsmtgs/ sectional.htm 1 .

\section{Invited Addresses}

Richard Kenyon, Brown University, Title to be announced.

Tony Pantev, University of Pennsylvania, Title to be announced.

Lai-Sang Young, New York University, Title to be announced.

\section{Gainesville, Florida}

\section{University of Florida}

\section{November 2-3, 2019}

Saturday - Sunday

\section{Meeting \# 1152}

Southeastern Section

Associate secretary: Brian D. Boe

Announcement issue of Notices: September 2019

Program first available on AMS website: September 19, 2019

Issue of Abstracts: Volume 40, Issue 4

\section{Deadlines}

For organizers: April 2, 2019

For abstracts: September 10, 2019

The scientific information listed below may be dated. For the latest information, see www.ams.org/amsmtgs /sectional.html. 


\section{MEETINGS \& CONFERENCES}

\section{Invited Addresses}

Jonathan Mattingly, Duke University, To be announced. Isabella Novik, University of Washington, To be announced.

Eduardo Teixeira, University of Central Florida, To be announced.

\section{Special Sessions}

If you are volunteering to speak in a Special Session, you should send your abstract as early as possible via the abstract submission form found at www. ams. org/cgi-bin /abstracts/abstract.p1.

Geometric and Topological Combinatorics (Code: SS 1A), Bruno Benedetti, University of Miami, Steve Klee, Seattle University, and Isabella Novik, University of Washington.

\section{Riverside, California}

\section{University of California, Riverside}

\section{November 9-10,2019}

Saturday - Sunday

\section{Meeting \# 1153}

Western Section

Associate secretary: Michel L. Lapidus

Announcement issue of Notices: September 2019

Program first available on AMS website: September 12, 2019

Issue of Abstracts: Volume 40, Issue 4

\section{Deadlines}

For organizers: April 9, 2019

For abstracts: September 3, 2019

The scientific information listed below may be dated. For the latest information, see www. ams.org/amsmtgs /sectional.html.

\section{Invited Addresses}

Robert Boltje, University of California, Santa Cruz, Title to be announced.

Jonathan Novak, University of California, San Diego, Title to be announced.

Anna Skripka, University of New Mexico, Albuquerque, Title to be announced.

\section{Special Sessions}

If you are volunteering to speak in a Special Session, you should send your abstract as early as possible via the abstract submission form found at www. ams.org/cgi-bin /abstracts/abstract.p1.

Inverse Problems (Code: SS 3A), Hanna Makaruk, Los Alamos National Laboratory, and Robert Owczarek, Uni- versity of New Mexico, Albuquerque and University of New Mexico, Los Alamos.

Random Matrices and Related Structures (Code: SS 2A), Jonathan Novak, University of California, San Diego, and Karl Liechty, De Paul University.

Topics in Operator Theory (Code: SS 1A), Anna Skripka and Maxim Zinchenko, University of New Mexico.

\section{Denver, Colorado}

\section{Colorado Convention Center}

January 15-18,2020

Wednesday - Saturday

\section{Meeting \# 1154}

Joint Mathematics Meetings, including the 126th Annual Meeting of the AMS, 103rd Annual Meeting of the Mathematical Association of America (MAA), annual meetings of the Association for Women in Mathematics (AWM) and the National Association of Mathematicians (NAM), and the winter meeting of the Association of Symbolic Logic (ASL), with sessions contributed by the Society for Industrial and Applied Mathematics (SIAM)

Associate secretary: Michel L. Lapidus

Announcement issue of Notices: October 2019

Program first available on AMS website: November 1, 2019 Issue of Abstracts: To be announced

\section{Deadlines}

For organizers: April 1, 2019

For abstracts: To be announced

\section{Charlottesville, Virginia}

\section{University of Virginia}

\section{March 13-15, 2020}

Friday - Sunday

Southeastern Section

Associate secretary: Brian D. Boe

Announcement issue of Notices: To be announced Program first available on AMS website: To be announced Issue of Abstracts: To be announced

\section{Deadlines}

For organizers: To be announced For abstracts: To be announced 


\section{Fresno, California}

\section{California State University, Fresno}

\section{May 2-3, 2020}

Saturday - Sunday

Western Section

Associate secretary: Michel L. Lapidus

Announcement issue of Notices: To be announced

Program first available on AMS website: To be announced Issue of Abstracts: To be announced

\section{Deadlines}

For organizers: To be announced

For abstracts: To be announced

\section{El Paso, Texas}

\section{University of Texas at El Paso}

\section{September 12-13,2020}

Saturday - Sunday

Central Section

Associate secretary: Georgia Benkart

Announcement issue of Notices: To be announced

Program first available on AMS website: To be announced Issue of Abstracts: To be announced

\section{Deadlines}

For organizers: To be announced

For abstracts: To be announced

\section{Washington, District of Columbia}

Walter E. Washington Convention Center

January 6-9, 2021

Wednesday - Saturday

Joint Mathematics Meetings, including the 127th Annual Meeting of the AMS, 104th Annual Meeting of the Mathematical Association of America (MAA), annual meetings of the Association for Women in Mathematics (AWM) and the National Association of Mathematicians (NAM), and the winter meeting of the Association of Symbolic Logic (ASL), with sessions contributed by the Society for Industrial and Applied Mathematics (SIAM).

Associate secretary: Brian D. Boe

Announcement issue of Notices: October 2020

Program first available on AMS website: November 1, 2020 Issue of Abstracts: To be announced

\section{Deadlines}

For organizers: April 1, 2020

For abstracts: To be announced

\section{Grenoble, France}

\section{Université Grenoble Alpes}

July 5-9, 2021

Monday - Friday

Associate secretary: Michel L. Lapidus

Announcement issue of Notices: To be announced Program first available on AMS website: To be announced Program issue of electronic Notices: To be announced Issue of Abstracts: To be announced

\section{Deadlines}

For organizers: To be announced For abstracts: To be announced 


\section{MEETINGS \& CONFERENCES}

\section{Buenos Aires,} Argentina

The University of Buenos Aires

July 19-23, 2021

Monday - Friday

Associate secretary: Steven H. Weintraub

Announcement issue of Notices: To be announced

Program first available on AMS website: To be announced Issue of Abstracts: To be announced

\section{Deadlines}

For organizers: To be announced

For abstracts: To be announced

\section{Seattle, Washington}

\section{Washington State Convention Center and} the Sheraton Seattle Hotel

\section{January 5-8, 2022}

Wednesday - Saturday

Associate secretary: Georgia Benkart

Announcement issue of Notices: October 2021

Program first available on AMS website: To be announced Issue of Abstracts: To be announced

\section{Deadlines}

For organizers: To be announced For abstracts: To be announced

\section{Boston, Massachusetts}

John B. Hynes Veterans Memorial Convention Center, Boston Marriott Hotel, and Boston Sheraton Hotel

January 4-7, 2023

Wednesday - Saturday

Associate secretary: Steven H. Weintraub Announcement issue of Notices: October 2022 Issue of Abstracts: To be announced

\section{Deadlines}

For organizers: To be announced For abstracts: To be announced

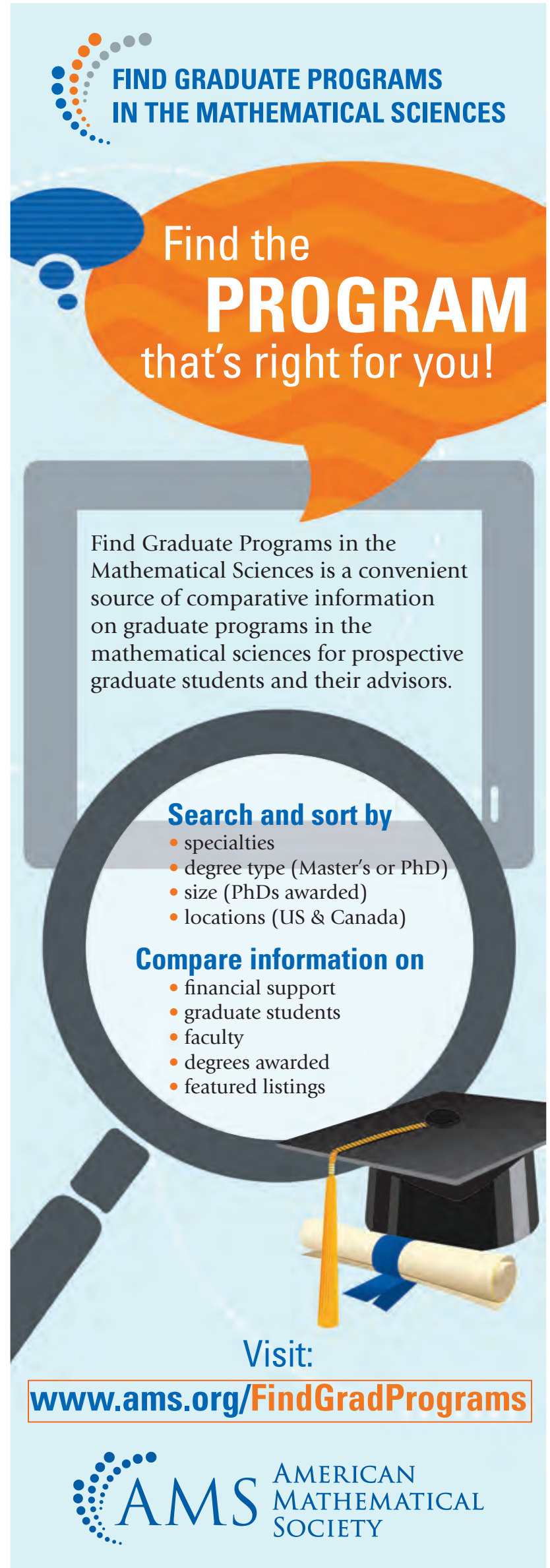



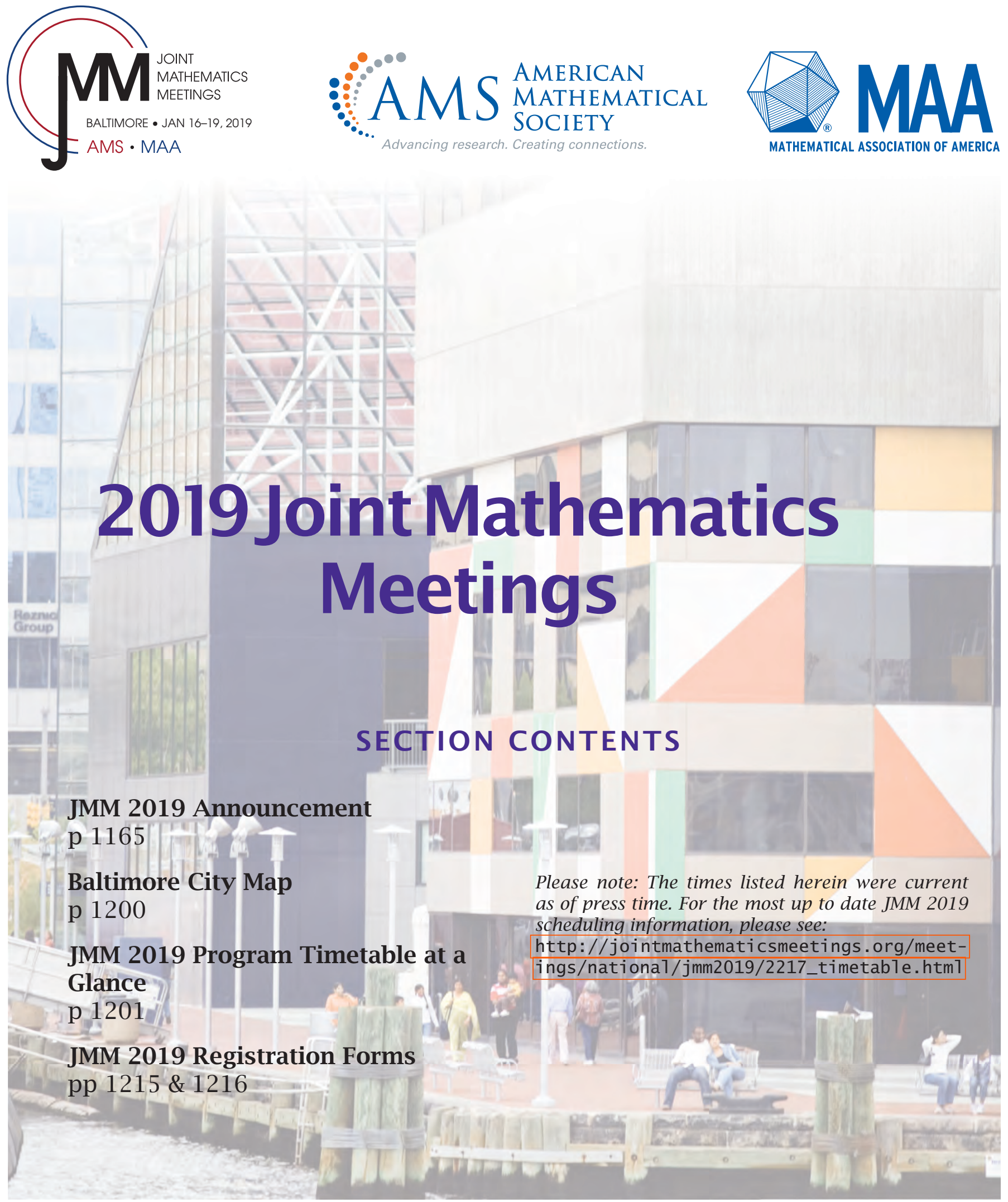


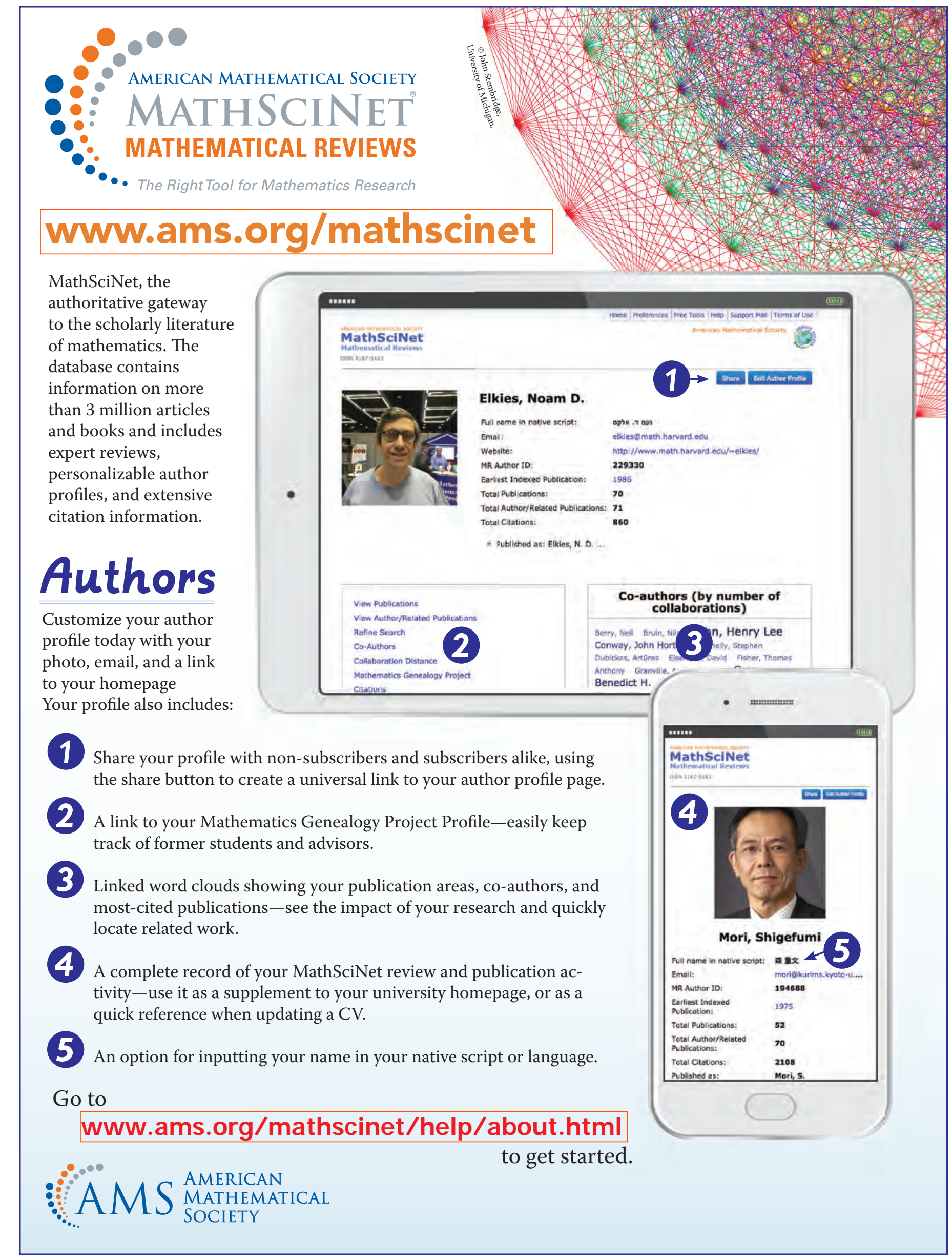



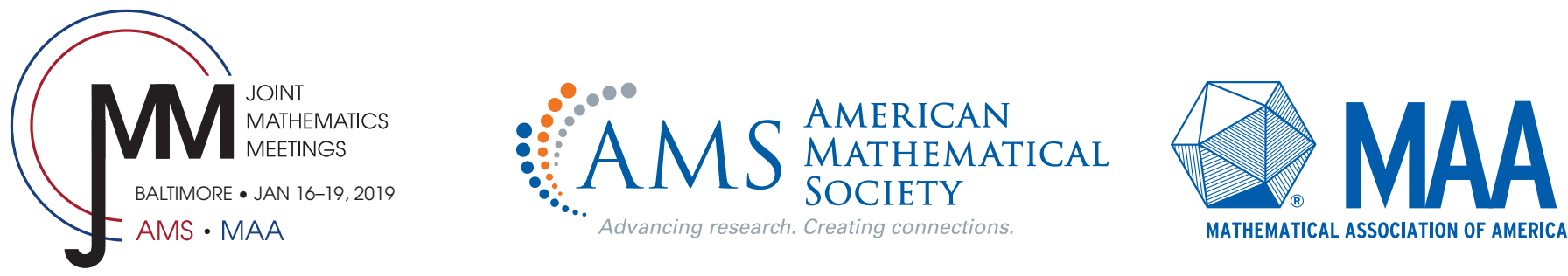

The American Mathematical Society and the Mathematical Association of America invite you to join them at the largest mathematics meeting in the world when it returns to Baltimore in January!

See https://jointmathematicsmeetings.org/meetings/national/jmm2019/2217_intro for the latest on JMM 2019

\section{Baltimore, MD}

Baltimore Convention Center, Hilton Baltimore, and

\section{Marriott Inner Harbor}

January 16-19, 2019

Wednesday-Saturday

\section{Meeting \# 1145}

Joint Mathematics Meetings, including the 125th Annual Meeting of the AMS, 102nd Annual Meeting of the Mathematical Association of America (MAA), annual meetings of the Association for Women in Mathematics (AWM) and the National Association of Mathematicians (NAM), and the winter meeting of the Association of Symbolic Logic (ASL), with sessions contributed by the Society for Industrial and Applied Mathematics (SIAM).

AMS Associate Secretary: Steven Weintraub

MAA Associate Secretary: Tensia Soto

Announcement issue of Notices: October 2018

Program first available on AMS website: To be announced

\section{Deadlines}

For organizers: Expired

For abstracts: September 25, 2018

The scientific information listed below may be dated. For the latest information, see www.ams.org/meetings /national.html.

\section{Joint Invited Addresses}

Sarah Koch, University of Michigan, What is the shape of a rational map? (AMS-MAA Invited Address); Wednesday, 11:10 am.

Cathy O'Neil, CEO of ORCAA, Big data, inequality, and democracy, (MAA-AMS-SIAM Gerald and Judith Porter Public Lecture); Saturday, 3:00 pm.
Daniel Spielman, Yale University, Miracles of algebraic graph theory (AMS-MAA Invited Address);Friday, 11:10 am.

Bryna Kra, Northwestern University, Dynamics of systems with low complexity (AWM-AMS Noether Lecture); Thursday, 10:05 am.

\section{Joint Prize Session}

In order to showcase the achievements of recipients of the various prizes, the AMS and MAA are co-sponsoring this event at 4:25 pm on Thursday. A cash bar reception will immediately follow. All participants are invited to attend. The AMS, MAA, and SIAM will announce the JPBM Communications Award winner. The AMS, MAA, and SIAM will award the Frank and Brennie Morgan Prize for Outstanding Research in Mathematics by an Undergraduate Student. The AMS and SIAM will announce the Norbert Wiener Prize in Applied Mathematics. The AMS will announce the Levi L. Conant Prize, Mary P. Dolciani Prize for Excellence in Research, AMS E.H. Moore Research Article, AMS David P. Robbins Prize, AMS Ruth Lyttle Satter Prize in Mathematics, the Leroy P. Steele Prizes, and the Oswald Veblen Prize in Geometry. The MAA will award the Chauvenet Prize, the Euler Book Prize, Deborah and Franklin Tepper Haimo Awards for Distinguished College or University Teaching of Mathematics, and the Yueh-Gin Gung and Dr. Charles Y. $\mathrm{Hu}$ Award for Distinguished Service to Mathematics. The AWM will present the Louise Hay Award for Contributions to Mathematics Education, the M. Gweneth Humphreys Award for Mentorship of Undergraduate Women in Mathematics, and the Birman Prize in Geometry and Topology. 


\section{5th Meeting of the AMS}

\section{AMS Invited Addresses}

Jesus A. De Loera, University of California, Davis, Algebraic, geometric, and topological methods in optimization; Wednesday, 10:05 am.

Peter Oszvath, Princeton University, Title to be announced, Thursday, 2:15 pm.

Lior Pachter, University of California Berkeley, Title to be announced, Thursday, 3:20 pm.

Alan Perelson, Los Alamos National Laboratory, Title to be announced (AMS Josiah Willard Gibbs Lecture); Wednesday, 8:30 pm.

Benedict H. Gross, University of California San Diego, Complex multiplication: past, present, future (AMS Colloquium Lectures: Lecture I), Wednesday, 1:00 pm.

Benedict H. Gross, University of California San Diego, Complex multiplication: past, present, future (AMS Colloquium Lectures: Lecture II); Thursday, 1:00 pm.

Benedict H. Gross, University of California San Diego, Complex multiplication: past, present, future (AMS Colloquium Lectures: Lecture III); Friday, 1:00 pm.

Karen Hunger Parshall, University of Virginia, The roaring twenties in American mathematics, Friday, 10:05 am.

Lillian Pierce, Duke University, Title to be announced; Saturday, 9:00 am.

\section{AMS Special Sessions}

If you are volunteering to speak in a Special Session, you should send your abstract as early as possible via the abstract submission form found at jointmathematicsmeetings.org/meetings /abstracts/abstract. p1?type=jmm.

Some sessions are co-sponsored with other organizations. These are noted within the parenthesis at the end of each listing, where applicable.

25 years of Conferences for African-American Researchers in the Mathematical Sciences (CAARMS times 25) (Code: SS 82A), William A. Massey, Princeton University.

A Showcase of Number Theory at Undergraduate Institutions (Code: SS 76A), Adriana Salerno, Bates College, and Lola Thompson, Oberlin College.

Advances and Applications in Integral and Differential Equations (Code: SS 40A), Jeffrey T. Neugebauer, Eastern Kentucky University, and Min Wang, Kennesaw State University.

Advances by Early Career Women in Discrete Mathematics (Code: SS 75A), Jessalyn Bolkema, State University of New York at Oswego, and Jessica De Silva, California State University, Stanislaus.

Advances in Operator Theory, Operator Algebras, and Operator Semigroups (Code: SS 30A), Joseph Ball, Virginia Tech, Marat Markin, California State University, Fresno, Igor Nikolaev, St. John's University, and Ilya Spitkovsky, New York University, Abu Dhabi.
Advances in Quantum Walks, Quantum Simulations, and Related Quantum Theory (Code: SS 18A), Radhakrishnan Balu, US Army Research Lab, Chaobin Liu, Bowie State University, and Takuya Machida, Nihon University, Japan.

Agent-based Modeling in Biological and Social Systems(a Mathematics Research Communities Session) (Code: SS 15A).

Algebraic Structures Motivated by Knot Theory (Code: SS 51A), Mikhail Khovanov, Columbia University, and Jozef H. Przytycki and Alexander Shumakovitch, George Washington University.

Algebraic and Geometric Methods in Discrete Optimization (Code: SS 2A), Amitabh Basu, Johns Hopkins University, and Jesus De Loera, University of California, Davis.

Algebraic, Discrete, Topological and Stochastic Approaches to Modeling in Mathematical Biology (Code: SS 43A), Olcay Akman, Illinois State University, Timothy D. Comar, Benedictine University, Daniel Hrozencik, Chicago State University, and Raina Robeva, Sweet Briar College.

Algorithmic Dimensions and Fractal Geometry (Code: SS 37A), Jack H. Lutz, Iowa State University, and Elvira Mayordomo, University of Zaragoza, Spain (AMS-ASL).

Analysis and Geometry of Nonlinear Evolution Equations (Code: SS 65A), Marius Beceanu, University at Albany, State University of New York, and Dan-Andrei Geba, University of Rochester.

Analysis of Fractional, Stochastic, and Hybrid Dynamic Systems with Applications (Code: SS 19A), John R. Graef, University of Tennessee at Chattanooga, G. S. Ladde, University of South Florida, and A. S. Vatsala, University of Louisiana at Lafayette.

Analytic Number Theory (Code: SS 33A), Thomas A. Hulse, Boston College, Angel V. Kumchev and Nathan McNew, Towson University, and John Miller, The Johns Hopkins University.

Arithmetic Statistics (Code: SS 53A), Michael Chou and Robert Lemke Oliver, Tufts University, and Ari Shnidman, Center for Communications Research-Princeton.

Bifurcations of Difference Equations and Discrete Dynamical Systems with Applications (Code: SS 72A), Arzu Bilgin, Recep Tayyip Erdogan University, Turkey, and Toufik Khyat, Trinity College.

Commutative Ring Theory: Research for Undergraduate and Early Graduate Students (Code: SS 60A), Nicholas Baeth, Franklin and Marshall College, and Branden Stone, Hamilton College.

Continued Fractions (Code: SS 35A), Geremías Polanco Encarnación, Hampshire College, James McLaughlin, West Chester University, Barry Smith, Lebanon Valley College, and Nancy J. Wyshinski, Trinity College.

Counting Methods in Number Theory (Code: SS 6A), Lillian Pierce, Duke University, Arindam Roy, Rice University, and Jiyua Wang, University of Wisconsin.

Definability and Decidability Problems in Number Theory (Code: SS 24A), Kirsten Eisenträger, Pennsylvania State University, Deidre Haskell, McMaster University, Ontario, Canada, Jennifer Park, University of Michigan, and Alexandra Shlapentokh, East Carolina University (AMS-ASL). 
Differential Equations on Fractals (Code: SS 87A), Patricia Alonso-Ruiz, University of Connecticut, Joe Chen, Colgate University, Luke Rogers, University of Connecticut, Robert Strichartz, Cornell University, and Alexander Teplyaev, University of Connecticut.

Enumerative Combinatorics (Code: SS 26A), Miklos Bona, University of Florida, and Cheyne Homberger, University of Maryland, Baltimore County.

Financial Mathematics (Code: SS 66A), Maxim Bichuch, Johns Hopkins University, Anja Richter, Baruch College, City University of New York, and Stephan Sturm, Worcester Polytechnic Institute.

Geometric and Topological Combinatorics (Code: SS 39A), Anastasia Chavez and Jamie Haddock, University of California, Davis, and Annie Raymond, University of Massachusetts, Amherst.

Geometric and Topological Generalization of Groups (Code: SS 74A), Amrita Acharyya, University of Toledo, and Bikash C. Das, University of North Georgia.

Geometry Labs United: Research, Visualization, and Outreach (Code: SS 57A), Marianne Korten, Kansas State University, and Sean Lawton and Anton Lukyanenko, George Mason University.

Geometry and Dynamics of Continued Fractions (Code: SS 47A), Anton Lukyanenko, George Mason University, and Joseph Vandehey, Ohio State University.

Geometry of Representation Spaces (Code: SS 58A), Sean Lawton, George Mason University, Chris Manon, University of Kentucky, and Daniel Ramras, Indiana UniversityPurdue University Indianapolis.

Group Representation Theory and Character Theory (Code: SS 38A), Mohammad Reza Darafsheh, University of Tehran, Iran, and Manouchehr Misaghian, Prairie View A\&M University.

Harmonic Analysis, Partial Differential Equations, and Applications (Code: SS 44A), Russell Brown, University of Kentucky, and Irina Mitrea, Temple University.

Harmonic Analysis: New Developments in Oscillatory Integrals(a Mathematics Research Communities Session) (Code: SS 12A), .

History of Mathematics (Code: SS 42A), Sloan Despeaux, Western Carolina University, Jemma Lorenat, Pitzer College, Daniel E. Otero, Xavier University, and Adrian Rice, Randolph-Macon College (AMS-MAA-ICHM).

Hopf Algebras and Tensor Categories (Code: SS 62A), Siu-Hung Ng, Louisiana State University, Julia Plavnik, Texas A\&M University, and Henry Tucker, University of California, San Diego.

How to Guard an Art Gallery and Other Discrete Mathematical Adventures (In Memory of T. S. Michael, 1960 to 2016) (Code: SS 16A), Joseph Bonin, The George Washington University, Carolyn Chun, US Naval Academy, and Nancy Neudauer, Pacific University.

If You Build It They Will Come: Presentations by Scholars in the National Alliance for Doctoral Studies in the Mathematical Sciences (Code: SS 54A), David Goldberg, Purdue University, and Phil Kutzko, University of Iowa.

Latinx in Math (Code: SS 34A), Alexander Diaz-Lopez, Villanova University, Laura Escobar, University of Illi- nois, and Juanita Pinzón-Caicedo, North Carolina State University.

Lattice Path Combinatorics and Applications (Code: SS 68A), Christian Krattenthaler, University of Vienna, Austria, and Alan Krinik and Randall J. Swift, California State Polytechnic University.

Localization and Delocalization for Disordered Quantum Systems (Code: SS 83A), Peter D. Hislop, University of Kentucky, Christoph A. Marx, Oberlin College, and Jeffery Schenker, Michigan State University.

Low Complexity Models in Data Analysis and Machine Learning (Code: SS 55A), Emily J. King, University of Bremen, Germany, Nate Strawn, Georgetown University, and Soledad Villar, New York University.

Mappings on Metric and Banach Spaces with Applications to Fixed Point Theory (Code: SS 63A), Torrey M. Gallagher, Bucknell University, and Christopher J. Lennard, University of Pittsburgh.

Mathematical Analysis in Fluid Dynamics (Code: SS 31A), Yanqiu Guo, Florida International University, Jinkai Li, South China Normal University, Jing Tian, Towson University, and Yuncheng You, University of South Florida.

Mathematical Investigations of Spatial Ecology and Epidemiology (Code: SS 79A), Leah Shaw and Junping Shi, College of William and Mary, and Zhisheng Shuai, University of Central Florida.

Mathematical Models in Ecology, Epidemiology, and Medicine (Code: SS 85A), Richard Schugart, Western Kentucky University, and Najat Ziyadi, Morgan State University.

Mathematicians at Sea (in the Sky, or on Land): Defense Applications of Mathematics (Code: SS 21A), Tegan Emerson, Timothy Doster, and George Stantchev, Naval Research Laboratory.

Mathematics in the Realm of Cyber Research (Code: SS 22A), Daniel Bennett, Army Cyber Institute, Paul Goethals, United States Military Academy, and Natalie Scala, Towson University.

Mathematics of Coding Theory and Applications (Code: SS 78A), Hiram Lopez-Valdez and Felice Manganiello, Clemson University, and Gretchen L. Matthews, Virginia Tech.

Mathematics of Gravity and Light (a Mathematics Research Communities Session) (Code: SS 11A), .

Multiscale Problems in the Calculus of Variations (Code: SS 46A), Elisa Davoli, University of Vienna, Austria, and Rita Ferreira, King Abdullah University of Science and Technology, Saudi Arabia.

Natural Resources Modeling (Code: SS 56A), Julie Blackwood, Williams College, and Shandelle M. Henson, Andrews University.

Network Science (Code: SS 52A), David Burstein, Swarthmore College, Franklin Kenter, United States Naval Academy, and Feng 'Bill' Shi, University of North Carolina.

New Directions in the Theory of Complex Multiplication (Code: SS 1A), Henri Darmon, McGill University, Samit Dasgupta, University of California, Santa Cruz, and Benedict Gross, Harvard University.

Nonlinear Evolution Equations and Their Applications (Code: SS 20A), Mingchao Cai, Morgan State University, 
Gisele Mophou Loudjom, University of French West Indies, Guadeloupe, France, and Gaston N'Guerekata, Alexander Pankov, Xuming Xie, and Guoping Zhang, Morgan State University.

Not KNerds: A Community for Knot Theory (Code: SS 77A), Moshe Cohen, Vassar College, Elizabeth Denne, Washington and Lee University, and Adam Lowrance, Vassar College.

Number Theoretic Methods in Hyperbolic Geometry (a Mathematics Research Communities Session) (Code: SS 14A), Samantha Fairchild, University of Washington, Junxian Li, University of Illinois Urbana Champaign, and Richard Vradenburgh, University of Virginia.

Number Theory, Arithmetic Geometry, and Computation (Code: SS 61A), Brendan Hassett, Brown University, Drew Sutherland, Massachusetts Institute of Technology, and John Voight, Dartmouth College.

Numerical Methods for PDEs and Applications (Code: SS 41A), Wenrui Hao, Qingguo Hong, and Jinchao Xu, Pennsylvania State University.

Optimal Methods in Applicable Analysis: Variational Inequalities, Low Rank Matrix Approximations, Systems Engineering, Cyber Security (Code: SS 81A), Aritra Dutta, King Abdullah University of Science and Technology, Saudi Arabia, Ram Mohapatra, University of Central Florida, Gayatri Pany, Singapore University of Technology and Design, Singapore, and Nabin Kumar Sahu, Dhirubhai Ambani Institute of Information and Communication Technology, India.

Orthogonal Polynomials, Quantum Probability, Harmonic and Stochastic Analysis (Code: SS 27A), Nobuhiro Asai, Aichi University of Education, Kariya, Japan, Rodica Costin, The Ohio State University, Aurel I. Stan, The Ohio State University at Marion, and Hiroaki Yoshida, Ochanomizu University, Tokyo, Japan.

Partition Theory and Related Topics (Code: SS 80A), Dennis Eichhorn, University of California, Irvine, Tim Huber, University of Texas, Rio Grande Valley, and Amita Malik, Rutgers University.

Problems in Partial Differential Equations (Code: SS 36A), Alex Himonas, University of Notre Dame, and Curtis Holliman, The Catholic University of America.

Quantum Symmetries: Subfactors and Fusion Categories (a Mathematics Research Communities Session) (Code: SS 13A), Cain Edie-Michell, Australian National University, Lauren Ruth, UC Riverside, and Yilong Wang, Ohio State University.

Quaternions (Code: SS 28A), Terrence Blackman, Medgar Evers College, City University of New York, and Johannes Familton and Chris McCarthy, Borough of Manhattan Community College, City University of New York.

Recent Advancements in Mathematical Modeling of Cancer (Code: SS 49A), Kamila Larripa, Humboldt State University, and Hwayeon Ryu, University of Hartford.

Recent Advances and Trends in Computable Structure Theory (in honor of J. Remmel) (Code: SS 64A), Jennifer Chubb, University of San Francisco, and Tim McNicholl, Iowa State University.
Recent Advances in Biological Modeling and Related Dynamical Analysis (Code: SS 69A), Joshi Raj Hem, Xavier University, and Yanyu Xiao, University of Cincinnati.

Recent Advances in Homological and Commutative Algebra (Code: SS 70A), Neil Epstein, George Mason University, Claudiu Raicu, Notre Dame University, and Alexandra Seceleanu, University of Nebraska.

Recent Advances in Inverse Problems and Imaging (Code: SS 25A), Kui Ren, University of Texas at Austin, and Yang Yang, Michigan State University.

Recent Advances in Regularity Lemmas (Code: SS 71A), Gabriel Conant, University of Notre Dame, Rehana Patel, and Julia Wolf, University of Bristol, UK.

Recent Progress in Multivariable Operator Theory (Code: SS 86A), Dmitry Kaliuzhnyi-Verbovetsky and Hugo Woerdeman, Drexel University.

Research in Mathematics by Early Career Graduate Students (Code: SS 84A), Marat Markin, Morgan Rodgers, Khang Tran, and Oscar Vega, California State University, Fresno.

Research in Mathematics by Undergraduates and Students in Post-Baccalaureate Programs (Code: SS 32A), Darren A. Narayan, Rochester Institute of Technology, Khang Tran, California State University, Fresno, Mark David Ward, Purdue University, and John Wierman, The Johns Hopkins University (AMS-MAA-SIAM).

Riordan Arrays (Code: SS 50A), Alexander Burstein and Dennis Davenport, Howard University, Asamoah Nkwanta, Morgan State University, Lou Shapiro, Howard University, and Leon Woodson, Morgan State University.

Statistical, Variational, and Learning Techniques in Image Analysis and their Applications to Biomedical, Hyperspectral, and Other Imaging (Code: SS 45A), Justin Marks, Gonzaga University, Laramie Paxton, Washington State University, and Viktoria Taroudaki, Eastern Washington University.

Stochastic Analysis and Applications in Finance, Actuarial Science and Related Fields (Code: SS 17A), Julius N. Esunge, University of Mary Washington, See Keong Lee, University of the Sciences, Malaysia, and Aurel I. Stan, The Ohio State University at Marion.

Stochastic Differential Equations and Applications (Code: SS 59A), Carey Caginalp, University of Pittsburgh. Symbolic Dynamics (Code: SS 9A), Van Cyr, Bucknell University, and Bryna Kra, Northwestern University.

The Mathematics of Historically Black Colleges and Universities (HBCUS) in the Mid-Atlantic (Code: SS 88A), Edray Goins, Purdue University, Janis Oldham, North Carolina A\&T, Talitha Washington, Howard University, and Scott Williams, University at Buffalo, State University of New York.

Topological Data Analysis: Theory and Applications (Code: SS 73A), Justin Curry, University at Albany, State University of New York, Mikael Vejdemo-Johansson, College of Staten Island, City University of New York, and Sara Kalisnik Verovsek, Wesleyan University.

Topology, Structure and Symmetry in Graph Theory (Code: SS 48A), Lowell Abrams, George Washington University, and Mark Ellingham, Vanderbilt University. 
Using Modeling to Motivate the Study of Differential Equations (Code: SS 29A), Robert Kennedy, Centennial High School, Ellicott City MD, Audrey Malagon, Virginia Wesleyan University, Brian Winkel, SIMIODE, Cornwall NY, and Dina Yagodich, Frederick Community College.

Women in Topology (Code: SS 67A), Jocelyn Bell, Hobart and William Smith Colleges, Rosemary Guzman, University of Chicago, Candice Price, University of San Diego, and Arunima Ray, Max Planck Institute for Mathematics, Germany.

\section{AMS Sessions for Contributed Papers}

There will be sessions of ten-minute contributed talks. Although an individual may present only one contributed paper at a meeting, any combination of joint authorship may be accepted, provided no individual speaks more than once on the program. Contributed papers will be grouped together by related subject classifications into sessions.

\section{Submission of Abstracts for AMS Sessions}

Authors must submit abstracts of talks through joint mathematicsmeetings.org/meetings/abstracts/ abstract.p1?type $=j \mathrm{~mm}$. Indicate the number of authors for the paper, click on the "New Abstract" button, and you will be taken to the submission form. Simply follow the step-by-step instructions until you receive your unique abstract receipt number. No submission is complete until you are given this number. The deadline for all submissions is September 25, 2018. Late papers cannot be accommodated. Please e-mail abs-coord@ams . org if you have questions. If you make an inquiry about your specific abstract, please include your abstract receipt number.

\section{Other AMS Sessions}

AMS Committee on the Profession Panel Discussion: Permanent teaching faculty in research oriented departments, Wednesday, 4:30-6:00 pm. There is a traditional view of research departments in mathematics where all or almost all the permanent faculty are involved in both teaching and research. For various reasons, in particular financial pressures coming from increasing enrollments and decreasing support, this is not the case at many institutions. It is important for the mathematical community to acknowledge this fact and to see the implications for the profession. The purpose of this panel is to give different perspectives on this issue, both looking at what departments look like now and how we might hope that they should develop.

AMS Education and Diversity Department Panel: Bridge-to-PhD and Postbac Programs Working to Open Doors for Students from Underrepresented Groups, organized by Helen G. Grundman, American Mathematical Society; Thursday, 2:45-4:15 pm. Postbac and bridge-to$\mathrm{PhD}$ programs in the mathematical sciences have been gaining popularity as a way to enable promising students who are not yet ready to enter a $\mathrm{PhD}$ program a pathway to successfully obtaining the degree. The panelists will describe four different currently-running one-semester to two-year bridge-to- $\mathrm{PhD} /$ postbac programs specifically designed to improve the diversity among Mathematics and Applied Mathematics PhDs. Although each of the programs offers students both advanced coursework and one-on-one mentoring in order to prepare them for success in graduate school, the programs differ in many other aspects. Discussion will include the strengths and weaknesses of each of these models, and any advice that the panelists or audience members have for mathematicians interested in starting new programs along these lines. Moderator for this panel will be Helen G. Grundman, American Mathematical Society. Panelists are Ruth Haas, University of Hawai'i, Ryan Hynd, University of Pennsylvania, and Trachette Jackson, University of Michigan, and Michael Young, Iowa State University.

AMS Committee on Education Panel Discussion, Thursday, 1:00-2:30 pm.

Grad School Fair, Friday, 8:30-10:30 am. Here is the opportunity for undergrads to meet representatives from mathematical sciences graduate programs from universities all over the country. January is a great time for juniors to learn more, and college seniors may still be able to refine their search. This is your chance for one-stop shopping in the graduate school market. At last year's meeting over 300 students met with representatives from more than 60 graduate programs. If your school has a graduate program and you are interested in participating, for US\$125 a table will be provided for your posters and printed materials (registration for this event must be made by a person already registered for the JMM), and you are welcome to personally speak to interested students. Complimentary coffee will be served. Co-sponsored by the AMS and MAA.

Current Events Bulletin, organized by David Eisenbud, Mathematical Sciences Research Institute; Friday, 1:00 pm-5:00 pm. Speakers in this session follow the model of the Bourbaki Seminars in that mathematicians with strong expository skills speak on work not their own. Written versions of the talks will be distributed at the meeting and will also be available online at www. ams . org/ams/current-events-bu1 1 etin.htm1 after the conclusion of the meeting.

AMS Committee on Science Policy Panel Discussion, organized by Anna Mazzucato, Pennsylvania State University, Ravi Vakil, Stanford University, and Michael Vogelius, Rutgers, The State University of New Jersey; Friday, 2:30-4:00 pm.

Congressional Fellowship Session, organized by Karen Saxe, American Mathematical Society; Friday, 4:30-6:30 $\mathrm{pm}$. This fellowship provides a public policy learning experience, demonstrates the value of science-government interaction and brings a technical background and external perspective to the decision-making process in Congress. Learn more about this program and speak with current and former AMS Fellows. James Ricci, AMS Congressional Fellow 2018-2019 will speak at this session. Application deadline for the 2018-19 AMS Congressional Fellowship is February 15, 2019. 
Who Wants to Be a Mathematician Championship, organized by Michael A. Breen, American Mathematical Society, and William T. Butterworth, DePaul University; Saturday, 1:00 pm-2:45 pm. Show your support for top high school students from the US, Canada, and the UK in this international Who Wants to Be a Mathematician as they compete for a US $\$ 5,000$ first prize for themselves and US $\$ 5,000$ for their school's math department. Semifinals are at 1:00 pm and finals are at 2:00 pm. Come match wits with the contestants, support their mathematical achievement, and have tremendous fun at the same time.

\section{Other AMS Events}

Council, Tuesday, 1:30 pm.

Business Meeting, Saturday, 11:45 am. The secretary notes the following resolution of the Council: Each person who attends a business meeting of the Society shall be willing and able to identify himself as a member of the Society. In further explanation, it is noted that each person who is to vote at a meeting is thereby identifying himself as and claiming to be a member of the American Mathematical Society. The Society has a Committee on the Agenda for Business Meetings. The purpose is to make business meetings orderly and effective. The committee does not have legal or administrative power. It is intended that the committee consider what may be called "quasipolitical" motions. The committee has several possible courses of action on a proposed motion, including but not restricted to:

(a) doing nothing,

(b) conferring with supporters and opponents to arrive at a mutually accepted amended version to be circulated in advance of the meeting,

(c) recommending and planning a format for debate to suggest to a business meeting,

(d) recommending referral to a committee, and

(e) recommending debate followed by referral to a committee.

There is no mechanism that requires automatic submission of a motion to the committee. However, if a motion has not been submitted through the committee, it may be thought reasonable by a business meeting to refer it rather than to act on it without benefit of the advice of the committee.

In order that a motion for this business meeting receive the service offered by the committee in the most effective manner, it should be in the hands of the AMS Secretary by December 13, 2018.

\section{AMS Short Course on Sum of Squares: Theory and Applications}

This two-day course will take place on the Monday and Tuesday before the meeting actually begins. It is organized by Pablo A. Parrilo, Massachusetts Institute of Technology, and Rekha R. Thomas, University of Washington.

Interest in the theory and application of sums of squares (SOS) polynomials has exploded in the last two decades, spanning a wide spectrum of mathematical disciplines from real algebraic geometry to convex geometry, combinatorics, real analysis, theoretical computer science, quantum information and engineering. SOS theory and applications are accessible to well-prepared undergraduate or beginning graduate students, as well as academics who wish to learn this material.

The origins of SOS polynomials are anchored in the 19th century by Hilbert's famous characterization of nonnegative polynomials that are SOS. In 1924 Artin gave an affirmative answer to Hilbert's 17th problem on whether all nonnegative polynomials were SOS of rational functions. From this the field of real algebraic geometry was born-the study of real solutions to polynomial systems. While real solutions of polynomials equations are considerably more complicated than their complex counterpart, their role in applications cannot be overstated. SOS polynomials have experienced a renaissance in the last few years following the work of Shor, Nesterov, Lasserre, and Parrilo that connected them to modern optimization via semidefinite programming. A diverse interdisciplinary community now exists around SOS polynomials with an array of conferences and research programs at many of the top institutions worldwide. While the theory is rich and fascinating with many open questions, the large array of applications are equally enticing, allowing for many different angles and access points to the field.

Lecture topics will include an Overview of SOS polynomials by Greg Blekherman, Georgia Institute of Technology; Lifts of Convex Sets, Hamza Fawzi, University of Cambridge; Engineering Applications, Georgina Hall, Princeton University; Theoretical Computer Science, Ankur Moitra, Massachusetts Institute of Technology; Algebraic Geometry, Mauricio Velasco, Los Andes University, and Geometry of Spectrahedra, Cynthia Vinzant, North Carolina State University. Problem set sessions are planned for both days, and speakers and organizers will be available to assist participants with these exercises.

There are separate registration fees to participate in this course. Advanced registration fees for members: US\$124; nonmember, US\$190; student, unemployed, or emeritus members US $\$ 72$. If you choose to register on-site, the fee for members will be US $\$ 158$; nonmembers US $\$ 225$, and student, unemployed, or emeritus members US\$93. On-site registration will take place Monday, January 14, 2018 at the Hilton Baltimore. Please see the complete Short Course announcement on page 1131 of this issue, or go to www. ams.org/short-course.

\section{Department Chairs Workshop}

This annual one-day workshop for department chairs and leaders is held on Tuesday, 8:00 am-6:30 pm, the day before the JMM actually begins, and is lead by Malcolm Adams, University of Georgia, Gloria Marí-Beffa, University of Wisconsin-Madison, Douglas Mupasiri, University of Northern Iowa, and Jennifer Zhao, University of Michigan-Dearborn.

This workshop will examine the chair's role in leading a department. The day will be structured to include and encourage networking and sharing of ideas amongst participants. 
There is a separate registration and fee to participate. For further information, please contact the AMS Washington Office at 401-455-4116 or amsdc@ams .org.

\section{2nd Meeting of the MAA}

\section{MAA Invited Addresses}

Amanda Folsom, Amherst College, Symmetry, almost, Wednesday, 2:15 pm.

Emmanuel Candes, Stanford University, Sailing through data: discoveries and mirages, Wednesday, 3:20 pm.

Edray Goins, Pomona College, The past 50 years of African Americans in the mathematical sciences, Thursday, 9:00 am.

David Bressoud, Macalester College, Reflections on teaching calculus for the first time, 45 times, Thursday, 11:10 am. (MAA Project NeXT Lecture on Teaching and Learning)

Pamela Harris, Williams College, A mathematical journey of culture, community, and collaboration, Friday, 9:00 am.

Annalisa Crannell, Franklin \& Marshall College, Drawing conclusions from drawing a square, Friday, 1:00 pm (MAA Lecture for Students).

Deanna Haunsperger, Carlton College, The Inclusion Principle: the importance of community in mathematics, Saturday, 10:00 am (Retiring Presidential Address).

\section{Presentations by MAA Teaching Award Recipients}

Friday, 2:30-3:45 pm, organized by MAA President Deanna Hausperger, Carleton College and MAA Secretary James Sellers, Pennsylvania State University. Winners of the Deborah and Franklin Tepper Haimo Awards for Distinguished College or University Teaching will give presentations on the secrets of their success.

\section{MAA Invited Paper Sessions}

Trends in Mathematical and Computational Biology, organized by Timothy Comar, Benedictine University, Alicia Prieto Langarcia, Youngstown State University, and Raina Robeva, Sweet Briar College; Wednesday, 8:00-10:50 am. Mathematical and computational biology encompasses a diverse range of biological phenomena and quantitative methods for exploring those phenomena. The pace of research at this junction continues to accelerate and substantial advancements in problems from gene regulation, genomics, phylogenetics, RNA folding, evolution, infectious disease dynamics, neuroscience, growth and control of populations, ecological networks, drug resistance modeling, and medical breakthroughs related to cancer therapies have increasingly ensued from utilizing mathematical and computational approaches. Our session on current trends will sample from this diversity of important questions from biology and medicine and their mathematical treatments, with a goal of maximizing the range of topics and research methods presented at the session. Mathematical approaches will include deterministic and stochastic continuous dynamical models, as well as finite dynamical systems and combinatorial and algebraic methods.

Building Successful Communities in Mathematics, organized by Deanna Haunsperger, Carlton College; Wednesday, 8:00-11:00 am. Mathematicians have always formed communities, but over the past couple decades, some groups have become more deliberate in the formation of communities to provide support and a mathematical home for their people. Whether those communities are structured around geographic area, mathematical field, gender, stage of career, or some other attribute, they can provide welcoming and supportive environments for their members and have become an important aspect of some folks' professional identities. Some of these programs have demonstrably aided in the persistence of members of underrepresented groups; examples of such programs will be featured in the session.

Using Research about Teaching and Learning to Inform the Preparation of Graduate Students to Teach, organized by Jack Bookman, Carlton College and Teri J. Murphy, University of Cincinnati; Wednesday, 2:15-3:15 $\mathrm{pm}$. Many groups within the mathematics community have called for increased attention to the preparation we provide to graduate students for their teaching responsibilities. These efforts are especially important because of the roles graduate students play in introductory mathematics instruction and the impact of experiences in introductory courses on student outcomes, enrollment, and retention rates in STEM majors. To support these efforts, the College Mathematics Instructor Development Source (CoMInDS) project, which is based at MAA and funded by NSF (DUE Award \#1432381), assists faculty in the design and implementation of professional development for mathematics graduate students. One component of this assistance has been a summer workshop at which mathematics faculty (who will be providing the professional development to mathematics graduate students) have opportunities to gain familiarity with research on the teaching and learning of mathematics, learn about available instructional materials and evaluation, and design their department's program.

As part of our efforts to provide this assistance to a wider audience in the mathematics community, this session will highlight key findings from research on the teaching and learning of mathematics and showcase activities for mathematics graduate student professional development. Presentations in the session will be tied to themes in the MAA's Instructional Practices (IP) Guide. Specifically, we will focus on information relevant to supporting mathematics graduate students to utilize approaches highlighted in the Classroom Practices section of the IP Guide: Fostering Student Engagement and Selecting Appropriate Mathematical Tasks.

Research in Improving Undergraduate Mathematical Sciences Education: Examples Supported by the National 
Science Foundation's IUSE: EHR Program, organized by Ron Buckmire, Karen Keene, Sandra Richardson, Talitha Washington, and Lee Zia, National Science Foundation (NSF); Directorate for Education and Human Resources (EHR); Division of Undergraduate Education (DUE); Thursday, 8:00-11:00 am. In this Invited Paper Session, research and findings will be presented from projects funded by the National Science Foundation Division of Undergraduate Education's Improving Undergraduate STEM Education (IUSE) Program. The purpose of this session is to provide a venue for the mathematical sciences community to share recent research from innovations related to undergraduate mathematical sciences.

The session will highlight research from ongoing IUSEfunded projects, with a focus on the study of the teaching and learning of undergraduate mathematical sciences. Session topics will include research findings from one or more of the following themes related to undergraduate mathematical sciences education: (1) Systemic structures to support effective teaching and broadening participation; (2) Curricular and pedagogical innovations to strengthen student experiences in mathematical sciences learning; and (3) Effective use of digital tools and other sources as teaching and learning resources. Because some projects are in early stages of project development and analysis, research findings may be preliminary.

Inspiring Diversity in Mathematics: Culture, Community, and Collaboration, organized by Pamela Harris, Williams College, Alicia Prieto Langarica, Youngstown State University, and Chad Topaz, Williams College; Thursday, 8:00-12:00 pm. Due to the historical lack of visibility of minority mathematicians, students may continue to believe that they cannot pursue scientific careers nor belong in the mathematical community. This is an issue we must address in order to diversify the sciences. The goals of this session are twofold: to discuss some current statistics concerning under-representation in the mathematical community and to present programs, opportunities, and pedagogical techniques that aim to address this under-representation.

Modular Forms: Aesthetics and Applications, organized by Amanda Folsom, Amherst College; Thursday, 1:00-4:00 pm. Modular forms are seemingly ubiquitous. Their inherent symmetries have driven research directions for over a century, and have led to major theoretical advances in mathematics and number theory, as well as applications to many diverse areas including mathematical physics, combinatorics, representation theory, and more. This session will feature speakers whose research has emphasized aesthetics and applications of modular forms and related functions. In particular, the work of many speakers relates to the more recent theory of and applications of harmonic Maass forms (2002-present), which are rooted in both original theory due to Maass from the 1950 s as well as the enigmatic work of Ramanujan from 1920 on his mock theta functions.

The Past 50 Years of African Americans in the Mathematical Sciences, organized by Edray Goins, Pomona College; Friday, 8:00-11:00 am. 2019 marks the 50th
Anniversary of the founding of the National Association of Mathematicians. NAM seeks is to promote excellence in the mathematical sciences for underrepresented minorities in general and African-Americans in particular. In the 50 years since the founding of NAM, we have seen many milestones. In 1995, the annual Conference for African American Researchers in the Mathematical Sciences (CAARMS) began at MSRI. In 1997, Scott Williams started the website "Mathematicians of the African Diaspora," and Kate Okikiolu bacame the first African American to win a Sloan Research Fellowship. In 2000, three African American Women graduated from the University of Maryland the first year any African American women had done so. In 2005, the bi-annual Infinite Possibilities Conference (IPC) began at Spelman College. In this session, we celebrate those African Americans who have advanced knowledge in the mathematical sciences through their research and commitment to community.

Mathematical Thinking for Modern Data Science Problems, organized by Rick Cleary, Babson College, and Diana Thomas, US Military Academy; Friday, 1:00-3:30 pm. With the increasing wealth of data obtained by sensors, internet, and smart phones, data analysis is filled with familiar terms like artificial intelligence and big data. Many schools have diversified their mathematics offerings to include modern topics like machine learning and big data analytics. But even with these tools available, real applications are challenging and typically require a mix of modern and traditional approaches. This is certainly the case when searching for rare events in large data sets. For example, how do we use big data to predict rare events that have huge impact, like a severe injury or a specific pregnancy complication? How do we identify individuals inside a noisy data environment who may want to stay hidden, like a terrorist? How does a pro sports franchise find the future all star currently not on the radar of scouts and coaches?

Speakers will detail the work they have done with subject area experts in fields like health, education, public policy, business, and sports science, our speakers present some unusual data science problems, describe why they are challenging, and propose solutions. We will show how fundamental mathematical concepts and problem solving skills relate to these important real world problems.

Beauty and Art from Research Mathematics, organized by Diana Davis, Swarthmore College; Saturday, 8:00-11:00 am. Research mathematics can produce beautiful pictures, beautiful objects, and even beautiful movies or other art forms. In this session, we will showcase some of the beautiful art that has been created as a byproduct of research mathematics. Each speaker will introduce their research, and then display the resulting art, explaining its mathematical significance and also allowing attendees to appreciate its beauty.

Research in Undergraduate Mathematics Education: Highlights from the Annual SIGMAA on RUME Conference, organized by Megan Wawro, Virginia Tech and Aaron Weinberg, Ithaca College; Saturday, 8:30-10:50 am. The purpose of the SIGMAA on Research in Undergraduate 
Mathematics Education (RUME) is to foster research on the teaching and learning of undergraduate mathematics and to provide a support network for those who participate in this area of research. Current research foci include insights regarding: students' understanding of concepts inundergraduate mathematics courses such as calculus, differential equations, linear algebra, real analysis, or abstract algebra; student and instructor engagement inmathematical practices that transcend particular content, such as defining and proving; and the impact of various instructional methods on equity and student learning. The 2019 MAA Invited Paper Session on Research in Undergraduate Mathematics Education will highlight exemplary current research in the field. In particular, it will showcase 4-6 research papers that were presented at the 21st Annual SIGMAA on RUME Conference, which took place in San Diego, CA in February 2018.

Mathematics and Policy, organized by Eric Marland and Rick Klima, Appalachian State University; Saturday, 1:00-4:40 pm. Mathematics and policy meet at an awkward intersection, a conflict of simplicity and rigor, imposing practicality on impossibility, and making transparent decisions in an uncertain world. But the purpose of bringing policy and mathematics together is to find a balance and increase communication and discourse along a complex interface. Many times we find success simply from arranging to have the right people in the room together. Rather than focus on one aspect of this interface, we propose a broader look into the politics of mathematics and the mathematics of policy.

Bringing together several groups who are interested in a common topic, but who have very different ideas on what the topic really is, can spark innovation and conversations that would not otherwise take place. From working directly with policy makers to understanding policies; from developing policy to critiquing policy. How can the mathematics of gerrymandering influence practicing policy makers? How can insights in one arena help craft policy in another? How can the interaction between scientists and policy makers change the way we present our results?

\section{MAA Minicourses}

MAA Minicourses are open only to persons who register for the Joint Meetings and pay the Joint Meetings registration fee in addition to the appropriate minicourse fee. The MAA reserves the right to cancel any minicourse that is undersubscribed. Participants should read the descriptions of each minicourse thoroughly as some require participants to bring their own laptops and special software; laptops will not be provided in any minicourse. The enrollment in each minicourse is limited to 50; the cost is US $\$ 100$.

Minicourse \#1. Mathematical Inquiry and Writing through Sports, presented by Eric Kahn, Bloomsburg University, and Tricia Muldoon Brown, Georgia Southern University, ; Part A, Wednesday, 9:00-11:00 am, and Part B, Friday, 9:00-11:00 am. Two evidence-supported strategies to facilitate effective learning of undergraduate mathematics, topics introductory through advanced in nature, are in- quiry and writing. This minicourse introduces participants to these two techniques via the real-world framework of sports. Together, participants and moderators will work through sample in-class activities used in introductory mathematics courses such as Math for the Liberal Arts and Introduction to Statistics which utilize a variety of sports in order to demonstrate learning with a mathematical inquiry approach. We will provide a session guiding participants on finding and manipulating actual sports data for use in their own projects and outline possible solutions for sample writing projects provided by the moderators. Finally, participants will spend time collaboratively designing their own inquiry-based activities and writing projects using the medium of sports. The minicourse will be run as an interactive workshop, so for the greatest impact, participants should bring a laptop with wireless internet accessibility.

Minicourse \#2. Start Teaching Statistics using $R$ and RStudio, presented by Randall Pruim, Calvin College and Shonda Kuiper, Grinnell College; Part A, Wednesday, 9:00-11:00 am, and Part B, Thursday, 9:00-11:00 am. $\mathrm{R}$ is a freely available language and environment for statistical computing and graphics that has become popular in academia and in many industries. But can it be used with students? This mini-course will introduce participants to teaching applied statistics courses using computing in an integrated way. The presenters will share an approach and some favorite examples for using $\mathrm{R}$ to teach statistics to undergraduates at all levels.

Topics will include adopting a "Less Volume, More Creativity" approach to provide novices with a powerful but manageable set of tools, workflow in the RStudio environment, data visualization, basic statistical inference using R, and using R Markdown to create documents that include both text and R output. Much of this will be facilitated using packages developed by Project MOSAIC, an NSF-funded project seeking to increase the use of modeling and computation throughout the undergraduate curriculum. This minicourse is designed to be accessible to those with little or no experience teaching with R, and will provide participants with skills, examples, and resources that they can use in their own teaching. Participants should bring a laptop to the session. Each participant will be given access to an RStudio server account, so it is not necessary to have R or RStudio installed on the laptop. A web browser and internet capability should suffice. This course is sponsored by the SIGMAA on Statistics Education (SIGMAA STAT ED).

Minicourse \#3. Advanced Authoring in WeBWorK: Turn good math problems into great ones \& submit them to the OpenProblemLibrary, presented by Marianna Bonanome, NYC College of Technology, Samar ElHitti, NYC College of Technology, Michael E. Gage, University of Rochester, and K. Andrew Parker, NYC College of Technology; Part A, Wednesday, 2:15-4:15 pm, and Part B, Friday, 1:00-3:00 pm. This minicourse provides advanced training in authoring WeBWorK questions and submitting the results to the OpenProblemLibrary. After reviewing basic authoring, including PGML, participants will practice 
customizing hints and error messages, creating scaffolded "classwork" problems, using "niceTables" for accessibility, creating "draggable proofs" and, customizing problems which interact with R, SageMath and/or Geogebra. We'll set up a local OPL and practice uploading problems and contributing them to the WeBWorK community OPL on github.com. We will also give directions for installing "WeBWorK-in-Docker" on your laptop thereby creating a private development site on your laptop, isolated from the rest of your work, which you can turn on or off at will. Anyone is welcome, but our focus is on those familiar with WeBWorK who want to strengthen their authoring ability and to contribute to WeBWorK's OpenProblemLibrary. This course is sponsored by MAA Committee on Technology in Mathematics Education.

Minicourse \#4. Teaching an Undergraduate Computational Science Course, presented by Joseph Eichholz and Allen Holder, Rose-Hulman Insitute of Technology; Part A, Wednesday, 2:15-4:15 pm, and Part B, Friday, 1:00-3:00 pm. This minicourse is designed to help participants offer a computational science/modeling course at their home institution. The minicourse will have three segments: (1) a short overview of curricular goals, what is computational science, how a computational science is different from a numerical analysis course; (2) discussion of several different course projects, including modeling aeronautic lift, modeling radiotherapy, signal processing, portfolio optimization and stock pricing, and modeling fluid flows; and (3) a survey of resources that are available to instructors, and discussion of best practices. Throughout the minicourse participants will be actively engaged in discussions and completing portions of the projects. A background in computational science is not assumed.

Minicourse \#5. IBL SIGMAA Minicourse: Introduction to Inquiry-Based Learning, presented by Susan Crook, Loras University, Eric Kahn, Bloomsberg University, Brian Katz, Augustana College, Victor Piercey, Ferris State University, Candice Price, University of San Diego, and Xiao Xiao, Utica College; Part A, Thursday, 9:00-11:00 am, and Part B, Saturday, 9:00-11:00 am. This minicourse will be a hands-on introduction to inquiry-based learning. Inquiry-based learning is a pedagogical approach that strongly emphasizes active learning and sense-making. During the minicourse, the facilitators and participants will model some typical IBL classroom modes as teachers and students and then reflect on and analyze these experiences. Discussion will include finding and using existing resources that support inquiry-based teaching and a variety of manners in which participants can use them to integrate some IBL practices into their classrooms. The minicourse is intended for new users of inquiry-based learning and for faculty who are interested in becoming new users. By the end, the participants will be familiar with resources and facilitation methods for using inquiry-based learning in the classroom.

Minicourse \#6. Visualizing Multivariable Calculus \& Differential Equations using CalcPlot3D, presented by Paul E. Seeburger, Monroe Community College, and Monica VanDieren, Robert Mor- ris University; Part A, Friday, 9:00-11:00 am, and Part B, Saturday, 1:00-3:00 pm. This course explores the use of CalcPlot3D to visualize topics in multivariable calculus and differential equations. CalcPlot3D is a versatile JavaScript web app for use on phones, tablets and computers, developed by the presenter through NSFIUSE \#1524968. Participants will use the app to visually verify problems from a variety of topics in both courses including the plane through 3 points, the intersection of two surfaces, the domain of a function of two variables, the general solution of a first-order differential equation, and general solutions of systems of differential equations. We will also explore a series of hands-on concept explorations designed to facilitate student understanding through exploration and visualization of a series of dynamic examples. Exploration topics include: dot product, cross product, velocity \& acceleration vectors, Lagrange multiplier optimization, planes and lines in space, translations and transformations of surfaces, and TNB frames. Participants will learn to customize this app to create dynamic demonstrations and guided exploration activities of their own for student use and create files for 3D printing surfaces from the app. Participants will receive 3D glasses. Bring a laptop. See https://sites . mon roecc . edu/multivariablecalculus/. This course is sponsored by WebSIGMAA.

Minicourse \#7. Using Data Applications to Inspire Linear Algebra Topics in the Classroom, presented by Tom Asaki, Washington State University, Amanda Harsy, Lewis University, Heather A. Moon, Lewis-Clark State College, and Marie A. Snipes, Kenyon College; Part A, Wednesday, 9:00-11:00 am, and Part B, Friday, 9:00-11:00 am. This minicourse is designed for participants who wish to incorporate data applications into their linear algebra courses. It provides a hands-on introduction to two data applications that inspire a host of linear algebra topics in the classroom: brain scan tomography (3d image reconstruction) and heat diffusion (diffusion welding and image warping). Participants will work with these applications using either Matlab or Octave, but no prior experience with these programs is required. There will also be time for pedagogical advice and group discussions regarding how to adapt the provided code and materials to one's own course.

Minicourse \#8. Dance and Mathematics, presented by Karl Schaffe, De Anza College; Part A, Thursday, 9:00-11:00 am, and Part B, Saturday, 9:00-11:00 am. In this Minicourse we will present several activities which combine dance and mathematics content in nontrivial ways. The activities connect to a variety of dance forms, as well as to several areas of mathematics, including symmetry, number theory, combinatorics, dynamical systems, and topology. Participants will take away activities useful in a wide range of undergraduate math classes or math clubs. The activities are collaborative and physically comfortable, and easily performed by those with little or no dance experience. These include folk dances, improvisations, and choreographic exercises with specific mathematical content, as well as kinesthetic tasks involving explorations of 
mathematical principles. In all cases, mathematics will illuminate the dance explorations, and the dance activity will realize, in kinesthetic form, the mathematical concepts.

Minicourse \#9. Mathematical Art from Complex Analysis, presented by Frank Farris, Santa Clara University; Part A, Wednesday, 2:15-4:15 pm and Part B, Thursday, 1:00-3:00 pm. Learn the theory and practice of making art with the domain-coloring algorithm, a distinctive approach to creating patterns from continuous, complex-valued functions. Students will learn to use the open-source SymmetryWorks software package (Windows and Mac), written by students at Bowdoin College, to turn their own photographs into rosettes, friezes, and wallpaper patterns. We also connect to the theory of complex variables, with visual connections to analytic and nonanalytic functions. Being able to make your own images will enhance your teaching of abstract algebra, complex variables, and many other courses; besides, it's fun!

Minicourse \#10. Object Based Learning and the Smithsonian Learning Lab, presented by Amy Shell-Gellasch, Eastern Michigan University; Part A, Thursday, 1:00-3:00 pm, and Part B, Allowing students to handle and study physical objects (object based learning) in the classroom enhances interest and understanding. However it is not always convenient or possible to bring physical objects into the classroom. In those cases, images, videos, even audio can provide the same pedagogical benefits. In particular, museum and library collections such as the vast Smithsonian holdings are a wealth of mostly untapped material for the classroom. In 2016 the Smithsonian Center for Learning and Digital Access launched the Smithsonian Learning Lab (SLL). The Learning Lab is a free online resource platform for educators and is ideal for objected based learning in and outside of class. College educators have used this platform in all courses of study. Choose from over 2 million images and resources at the Smithsonian or import materials from other sources. Create a collection of items and materials for your course that students then access in class or at home for discussion or assignments. Students can also create their own collections for assignments or portfolios.

In this four-hour minicourse, we will explore methods and resources for using object based learning in the classroom. Participants will then create a free Smithsonian Learning Lab login, learn how to access Smithsonian materials, and how to create a course collection of annotated materials from the Smithsonian collections and beyond for use in a class they teach.

Minicourse \#11. How to Run Successful Math Circles for Students and Teachers, presented by Jane H. Long, Stephen F. Austin State University, Gabriella Pinter, University of Wisconsin Milwaukee, and Diana White, University of Colorado Denver and National Association of Math Circles; Part A, Thursday, 1:00-3:00 pm, and Part B, Saturday, 8:00-10:00 am. Math Circles are a unique form of outreach through which mathematics professionals share their passion for mathematics with K-12 students and teachers. During a Math Circle, participants explore, create and communicate substantive mathematics, increase their problem-solving skills, and perhaps most importantly, develop a deeper enjoyment of the subject. Including all types of Math Circles, there are currently over 250 Math Circles across the United States. In this minicourse, participants will experience Math Circle activities and discuss related topics including effective facilitation of sessions, recruiting, logistics, and successful Math Circle models. Participants should be well on their way to starting their own Math Circle after this course. This course is sponsored by SIGMAA on Math Circles for Students and Teachers (SIGMAA-MCST).

Minicourse \#12. Keep Teaching Statistics using $\boldsymbol{R}$ and $\boldsymbol{R} S$ tudio, presented by presented by Randall Pruim, Calvin College and Shonda Kuiper, Grinnell College; Part A, Friday, 1:00-3:00 pm, and Part B, Saturday, 1:00-3:00 pm. This minicourse is intended for those who are already familiar with R and RStudio or who have coregistered for the Start Teaching Statistics using $\mathrm{R}$ and RStudio minicourse and will provide an introduction to additional aspects of $\mathrm{R}$ that are useful for teaching statistics and data science courses at a variety of levels. Topics will include data wrangling (obtaining data and transforming it into useful formats) using the tidyverse suite of tools, creating interactive instructional resources (e.g., with learnr tutorials or shiny documents), as well as additional modeling topics (such as simulation-based inference, machine learning, and visualizing models).

Participants should bring a laptop to the session. Each participant will be given access to an RStudio server account, so it is not necessary to have R or RStudio installed on the laptop. A web browser and internet capability should suffice. This course is sponsored by SIGMAA Stat Ed.

\section{MAA Contributed Papers}

The MAA Committee on Sessions of Contributed Papers solicits papers pertinent to the sessions listed below. Any paper that fits the subject of one of the themed sessions should be submitted directly to that session. All others should be submitted to the general sessions, which will accept abstracts in all areas of collegiate mathematics, mathematical pedagogy, and the undergraduate mathematics curriculum. Contributed Paper Session presentations in the themed sessions are limited to 15 minutes in length while presentations in the general sessions are limited to 10 minutes each. Potential submitters are advised to read the full descriptions of these sessions at jointmathematicsmeetings .org/meetings/nationa1/jmm2018/JMM2018_MAA _Ca11_for_Papers.pdf.

The deadline for submission of abstracts is Tuesday, September 25, 2018.

\section{MAA Contributed Paper Sessions with Themes} Approaches to Mathematics Remediation in Baccalaureate-Granting Institutions, organized by Michael Boardman, Pacific University, Helen E. Burn, Highline College, 
and Mary E. Pilgrim, Colorado State University, Saturday morning.

Discrete Mathematics in the Undergraduate Curriculum-Ideas and Innovations in Teaching, organized by John Caughman, Portland State University, Oscar Levin, University of Northern Colorado, and Elise Lockwood, Oregon State University, Wednesday morning and afternoon.

The EDGE (Enhancing Diversity in Graduate Education) program: Pure and Applied talks by Women Math Warriors, organized by Laurel Ohm, University of Minnesota, and Shanise Walker, Iowa State University, Thursday afternoon.

Discrete Mathematics in the Undergraduate Curriculum - Ideas and Innovations in Teaching, organized by Elise Lockwood, Oregon State University, John Caughman, Portland State University, and Art Duval, University of Texas El Paso; Wednesday afternoon.

Ethnomathematics: Ideas and Innovations in the Classroom, organized by Janet Beery, University of Redlands, Antonia Cardwell, Millersville University of Pennsylvania, Ximena Catepillan, Millersville University of Pennsylvania, and Amy Shell-Gellasch, Eastern Michigan University, Friday morning. Sponsored by the History of Mathematics SIGMAA.

Formative and Summative Assessment of Mathematical Communication and Conceptual Understanding, organized by Jessica OShaughnessy, Shenandoah University, and Jana Talley, Jackson State University, Thursday afternoon.

Fostering Creativity in Undergraduate Mathematics Courses, organized by Emily S. Cilli-Turner, University of Washington Tacoma, Houssein El Turkey, University of New Haven, Gulden Karakok, University of Northern Colorado, Milos Savic, University of Oklahoma, and Gail Tang, University of La Verne, Saturday morning.

Good Math from Bad: Crackpots, Cranks, and Progress, organized by Elizabeth T. Brown, James Madison University, and Samuel R. Kaplan, University of North Carolina Asheville, Friday afternoon.

Humanistic Mathematics, organized by Gizem Karaali, Pomona College, and Eric Marland, Appalachian State University, Thursday morning. Sponsored by the MAA Curriculum Renewal Across the First Two Years (CRAFTY) and the Journal of Humanistic Mathematics.

Inclusive Excellence-Attracting, Involving, and Retaining Women and Underrepresented Groups in Mathematics, organized by Francesca Bernardi, University of North Carolina at Chapel Hill, Meghan DeWitt, St Thomas Aquinas College, Semra Kilic-Bahi, Colby-Sawyer College, and Minah Oh, James Madison University, Saturday morning. Sponsored by the MAA Committee on the Participation of Women in Mathematics.

Incorporating Programming and Computing in the Math Major Curriculum, organized by Holly Peters Hirst, Appalachian State University, and Gregory S. Rhoads, Appalachian State University, Saturday morning.

Inequalities and Their Applications, organized by Titu Andreescu, University of Texas at Dallas, and Henry J. Ricardo, Westchester Area Math Circle, Thursday morning.
Infusing Data Science and Big Data into the Statistics Classroom, organized by Allen Harbaugh, Boston University, Wednesday afternoon. Sponsored by the SIGMAA on Statistics Education (SIGMAA STAT ED).

Innovative Curricular Strategies for Increasing Mathematics Majors, organized by Stuart Boersma, Central Washington University, Eric Marland, Appalachian State University, Victor Piercey, Ferris State University, and Frank Savina, University of Texas at Austin, Wednesday morning. Sponsored by the MAA Curriculum Renewal Across the First Two Years (CRAFTY).

Innovative and Effective Ways to Teach Linear Algebra, organized by Sepideh Stewart, University of Oklahoma, Gil Strang, Massachusetts Institute of Technology, David Strong, Pepperdine University, and Megan Wawro, Virginia Tech, Thursday morning.

Innovative Pathways to Quantitative Literacy, organized by Catherine Crockett, Point Loma Nazarene University, Keith Hubbard, Stephen F. Austin State University, and Jennifer Nordstrom, Linfield College, Saturday morning. Sponsored by the MAA Committee on Articulation and Placement MAA Subcommittee on Curriculum Renewal Across the First Two Year SIGMAA on Quantitative Literacy.

Inquiry-Based Learning and Teaching, organized by Susan Crook, Loras College, Eric Kahn, Bloomsburg University, Brian Katz, Augustana College, Amy Ksir, United States Naval Academy, Victor Piercey, Ferris State University, Candice Price, University of San Diego, and Xiao Xiao, Utica College, Friday morning and afternoon. Sponsored by the SIGMAA on Inquiry-Based Learning (IBL SIGMAA).

Integrated STEM Instruction in Undergraduate Math ematics, organized by Jeneva Clark, University of Tennessee, Knoxville, and Anant Godbole, East Tennessee State University, Thursday afternoon.

Integrating Research into the Undergraduate Classroom, organized by Timothy B. Flowers, Indiana University of Pennsylvania, and Shannon R. Lockard, Bridgewater State University, Wednesday afternoon.

Introducing Mathematical Modeling through Competitions, organized by Chris Arney, United States Military Academy, William C. Bauldry, Appalachian State University, and Amanda Beecher, Ramapo College, Thursday morning. Sponsored by COMAP and SIMIODE.

It's Circular: Conjecture, Compute, Iterate, organized by Thomas J. Clark, Dordt College, and James Taylor, Math Circles Collaborative of New Mexico, Friday afternoon. Sponsored by SIGMAA-MCST.

Mathematical Experiences and Projects in Business, Industry, and Government (BIG), organized by Robert Burks, Naval Postgraduate School, and Allen Butler, Wagner Associates, Friday morning. This session is sponsored by the SIGMAA on Business, Industry, and Government. Sponsored by the Business Industry Government Special Interest Group of the Mathematical Association of America (BIG SIGMAA).

Mathematical Themes in a First-Year Seminar, organized by Jennifer Bowen, College of Wooster, Mark Kozek, 
Whittier College, Pamela Pierce, College of Wooster, and Jennifer Schaefer, Dickinson College, Friday afternoon.

Mathematics and the Arts, organized by Karl Kattchee, University of Wisconsin-LaCrosse, Douglas Norton, Villanova University, and Anil Venkatesh, Ferris State, Wednesday morning and afternoon. Sponsored by SIGMAA-ARTS.

Mathematics and the Life Sciences: Initiatives, Programs, Curricula, organized by Timothy D. Comar, Benedictine University, Carrie Diaz Eaton, Unity College, and Raina Robeva, Sweet Briar College, Thursday morning. Sponsored by the SIGMAA on Mathematical and Computational Biology (BIO SIGMAA).

Mathematics and Sports, organized by John David, Virginia Military Institute, and Drew Pasteur, College of Wooster, Wednesday afternoon. Sponsored by the Sports SIGMAA.

Meaningful Modeling in the First Two Years of College, organized by William C. Bauldry, Appalachian State University, and Mary R. Parker, Austin Community College, Saturday afternoon. Sponsored by the MAA Mathematics Across the Disciplines (MAD) Subcommittee and the MAA Curriculum Renewal Across the First Two Years (CRAFTY) Subcommittee.

Open Educational Resources: Combining Technological Tools and Innovative Practices to Improve Student Learning, organized by Benjamin Atchison, Framingham State University, Marianna Bonanome, New York City College of Technology, Margaret Dean, Borough of Manhattan Community College, Michael Gage, University of Rochester, and Annie Han, Borough of Manhattan Community College, Friday morning. Sponsored by the MAA Committee on Technologies in Mathematics Education (CTiME).

Philosophy of Mathematics, organized by Jeffrey Buechner, Rutgers University - Newark, and Bonnie Gold, Monmouth University (retired), Friday morning. Sponsored by POMSIGMAA.

Research in Undergraduate Mathematics Education (RUME), organized by Stacy Brown, California State Polytechnic University, Megan Wawro, Virginia Tech, and Aaron Weinberg, Ithaca College, Thursday morning and afternoon, and Friday morning and afternoon. Sponsored by SIGMAA RUME.

Revitalizing Complex Analysis, organized by Michael Brilleslyper, United States Air Force Academy, Russell Howell, Westmont College, and Beth Schaubroeck, United States Air Force Academy, Thursday afternoon.

The Scholarship of Teaching and Learning in Collegiate Mathematics, organized by Tom Banchoff, Brown University, Curtis Bennett, California State University, Long Beach, Pam Crawford, Jacksonville University, Jacqueline Dewar, Loyola Marymount University, Edwin Herman, University of Wisconsin-Stevens Point, and Lew Ludwig, Denison University, Wednesday morning \& afternoon.

The Teaching and Learning of Undergraduate Ordinary Differential Equations, organized by Christopher S. Goodrich, Creighton Preparatory School, and Beverly H. West, Cornell University, Friday afternoon. Sponsored by CODEE (Consortium of Ordinary Differential Equations Educators).

Technology and Resources in Statistics Education, organized by Stacey Hancock, Montana State University, and Karl RB Schmitt, Valparaiso University, Friday afternoon. Sponsored by the Committee on Technology in Mathematics Education SIGMAA-Statistics Education.

Touch it, Feel it, Learn it: Tactile learning activities in the undergraduate mathematics classroom, organized by Chris Oehrlein, Oklahoma City Community College, Ann Trenk, Wellesley College, and Laura Watkins, Glendale Community College, Thursday afternoon. Sponsored by the Professional Development committee and the Committee on Two-Year Colleges.

Undergraduate Student TAs in Mathematics, organized by Aaron Peterson and Ursula Porod, Northwestern University, Wednesday afternoon.

General Contributed Paper Sessions, organized by Emelie Kenney, Sienna College, and Melvin Royer, Indiana Wesleyan University; Wednesday, Thursday, Friday, and Saturday, mornings and afternoons. These sessions accept contributions in all areas of mathematics, curriculum, and pedagogy. When you submit your abstract you will be asked to classify it according to the following scheme: Assessment; History or Philosophy of Mathematics; Interdisciplinary Topics in Mathematics; Mathematics and Technology; Mentoring; Modeling and Applications; Outreach; Teaching and Learning Developmental Mathematics; Teaching and Learning Introductory Mathematics; Teaching and Learning Calculus; Teaching and Learning Advanced Mathematics; Algebra; Analysis; Applied Mathematics; Geometry; Graph Theory; Linear Algebra; Logic and Foundations; Number Theory; Probability and Statistics; Topology; and Other Topics.

See also the AMS-MAA-SIAM Special Session on Research in Mathematics by Undergraduates and Students in Post-Baccalaureate Programs, organized by Darren A. Narayan, Rochester Institute of Technology, Khang Tran, California State University, Fresno, Mark David Ward, Purdue University, and John Wierman, The Johns Hopkins University.

\section{Submission Procedures for MAA Contributed Paper Abstracts}

Abstracts may be submitted electronically at jointmathematicsmeetings.org/meetings/abstracts /abstract.p1?type =jmm. Simply fill in the number of authors, click "New Abstract," and then follow the step-bystep instructions. The deadline for abstracts submission is Tuesday, September 25, 2018.

Each participant may make at most one presentation in an MAA Contributed Paper Session, either a presentation in one of the themed sessions or a presentation in one of the general sessions. If a paper cannot be accommodated in the themed session for which it was submitted, it will automatically be considered for the general contributed paper sessions. The organizer(s) of your session will automatically receive a copy of the abstract, so it is not necessary for you to send it directly to the organizer. The 
session rooms are equipped with computer projectors and screens. Please note that the dates and times scheduled for these sessions remain tentative. Questions concerning the submission of abstracts should be addressed to abs-coord@ams .org.

\section{MAA Panels}

MAA Panel: Mathematics Placement Trends and Innovations that Increase Equitable Access \& Success, organized by James Ham, Delta College, Keith Hubbard, Stephen F. Austin State University, and Kathryn Lineham, Montgomery College, Wednesday, 9:35-10:55 am. The placement of students into college-level mathematics classes has been the topic of interest for higher education systems and has resulted in fundamental policy changes from state legislatures. In Florida, all remedial classes have been deemed optional. In California, multiple measures are now required for placement. In Texas, 75 percent of remedial students must be placed into co-requisite credit-level courses. All of these trends are intended to increase equitable access and success in mathematics course work. Come join this discussion of the approaches that are sweeping the nation and the effects they are having on institutions and students. Panelists are David Bressoud, Macalester College, Mary Margarita Legner, Riverside Community College, Jeremy Martin, Charles A. Dana Center, UT Austin, and Judith Scott-Clayton, Columbia University. This event is sponsored by the MAA on Committee on Articulation and Placement.

MAA Panel: What Every Student Should Know about the JMM, organized by Peri Shereen, California State University, Monterey Bay Violeta Vasilevska, Utah Valley University; Wednesday, 9:00-10:20 am. Navigating a large conference can be overwhelming, even for those who have previously attended such an event. Panelists Joyati Debnath, Winona State University; Matt DeLong, Marian University, and Zsuzsanna Szaniszlo, Valparaiso University, will provide guidance for students attending the Joint Mathematics Meetings, including answers to some common questions: How do I get the most out of the program? What sessions are especially for students? What other events should I be on the lookout for? Will I understand any of the invited addresses or should I not bother attending them? If I am presenting a poster, where do I go to set it up? How can I get some cool, free math stuff? Students and their faculty mentors are encouraged to attend. Panelists are Joyati Debnath, Winona State University, Matt DeLong, Marian University, and Zsuzsanna Szaniszlo, Valparaiso University. This panel is sponsored by the MAA Committee for Undergraduate Student Activities and Chapters (CUSAC).

MAA Panel: Mental Health in the Mathematics Profession, organized by Justin Curry, SUNY Albany, and Mikael Vejdemo-Johansson, CUNY College of Staten Island; Wednesday, 2:15-3:35 pm. Mental health problems - depression, anxiety, to mention some of the most common - strike about $18.5 \%$ of the population at large in any given year - chances are good that most mathematicians have had, or have colleagues who have had struggles.
The numbers only rise after including neurodivergences that often produce these as symptoms. The pressures of academia can exacerbate or cause such problems, causing distress and derailinbg careers. Visibility is low - we often hide or minimize our struggles, so as to not impact careers or the impressions we make on everyone else - and this further isolates anyone struggling: it can often feel like nobody else has any similar problems to overcome, and many issues get harder to handle with this isolation and added pressure. As a first step in building up visibility and creating a more supportive community for ourselves, we have gathered mathematicians at different stages of their careers with immediate personal experiences to a panel discussion on what life in mathematics with mental health problems is like, how we are handling it, what works and what doesn't. Panelists are Julie Corrigan, Charleston, Justin Curry, SUNY Albany, Kate Farinholt, National Alliance on Mental Illness, Rachel Levanger, University of Pennsylvania, and Mikael Vejdemo-Johansson, CUNY College of Staten Island.

MAA Panel: Pursuing New Directions in Your Academic Career, organized by Louis Deaett, Quinnipiac University, Linda McGuire, Muhlenberg College, Steven Schlicker, Grand Valley State University, and David Torain, Montgomery College, Wednesday, 2:153:35 pm. This panel is for faculty members who are looking for new directions to pursue within their academic careers. Panelists will share lessons learned from navigating new directions in their own careers, including adopting new teaching methodologies, exploring new areas of scholarship, engaging in service outside of one's institution, and changing academic positions (e.g., to administration or to another institution). Panelists are Curtis Bennett, California State University, Long Beach, Jill Guerra, University of Arkansas at Fort Smith, Ron Taylor, Berry College, and Suzanne Weekes, Worcester Polytechnic Institute. This event is sponsored by the MAA Committee on Professional Development.

MAA Panel: Impacting Mathematics Instruction Through Meaningful Collaboration with Partner Discipline Faculty, organized by Janet Bowers, San Diego State University; Wednesday, 4:155:35 pm. Mathematics is used throughout the undergraduate curriculum. Introductory mathematics courses, as well as the use of mathematics in other courses, would benefit from collaboration among faculty from mathematics and partner disciplines. Yet, how often do faculty from mathematics and the partner disciplines engage in meaningful conversation about the mathematics taught and used in the undergraduate curriculum? In the Curriculum Foundations Project (CFP), the Mathematical Association of America conducted a series of 22 workshops to facilitate such discussions. Reports of their findings appear in two volumes: tinyur1.com/CFPVoices and /tinyur1.com /CFPPartners. In the ongoing NSF supported SUMMIT-P project, ten institutions are successfully revising their introductory mathematics curriculum by building partnerships with partner disciplines using CFP findings. Panelists include two mathematicians and one faculty member each 
from engineering, chemistry and social science, each of whom has successfully collaborated through SUMMIT-P to improve instruction and student learning. Attendees will learn more about CFP findings, experience a "Fishbowl" discussion, and learn strategies for using these findings and facilitating cross-disciplinary conversations on their campus. Panelists are Susan L. Ganter, Embry-Riddle Aeronautical University, Rosalyn H. Hargraves, Virginia Commonwealth University, Stella Hofrenning, Augsburg University, Victor I. Piercey, Ferris State University, and Kathy Williams, San Diego State University.

MAA Panel: Connecting High School and Post High School Mathematics, organized by Gail Burrill, Michigan State University; Thursday, 9:00-10:20 am. NCTM's recently released document Catalyst for Change contains recommendations for the essential mathematical concepts that should be in the curriculum for all students and potential curricular pathways through four years of high school mathematics. The session will consider the implications of these recommendations for college intending students, how the recommendations intersect with the changing landscape of introductory courses in mathematics at an increasing number of post high school institutions, and ways to promote dialogue between instructors at these institutions that will help make the transition from high school mathematics to college mathematics one that is supportive of students and their goals. Time will be allocated to engage the audience in a discussion of important themes and next steps. Panelists are Dave Barnes, National Council of Teachers of Mathematics, Gail Burrill, Michigan State University, Karen Graham, University of New Hampshire, Yvonne Lai, University of Nebraska, Dan Teague, North Carolina School of Science and Mathematics, and Francis Su, Harvey Mudd College.

MAA Panel: Preparing Math and Stats Students for Industry Careers, organized by Namyong Lee, Minnesota State University, Mankato, Debra Mimbs, Lee University, and Thomas Wakefield, Youngstown State University; Thursday, 9:0010:20 am. There are hundreds of nonacademic jobs for math majors. However, most faculty members have little to no experience with or background in nonacademic careers. Panelists will discuss their experiences in preparing and advising students for nonacademic careers including some who participated in the MAA and NSF funded program known as PIC Math. Topics could include how to organize a semester course centered on solving research problems from industry, how to prepare students for nonacademic careers, how to initiate contact and develop relationships with local industries to get research problems and internships for students, how to effectively advise students for internship and career opportunities, examples of specific research problems from industry for students, as well as insights from former faculty now working in industry. Panelists include former faculty members who now work in business, industry or government as well as current faculty members who advise student projects in these areas. Panelists are Allen Butler, Daniel H. Wagner Associates, Inc, Michael Dorff, Brigham Young University, Gretchen
Koch-Noble, Federal Government, Aaron Luttman, Department of Energy, and Debra Mimbs, Lee University.

MAA Panel: Coping Professionally with Unprofessional Behavior, organized by Zsuzsanna Szaniszlo, Valparaiso University, and Jennifer Beineke, Western New England University; Thursday, 1:00-2:20 pm. Academia reflects real life in most aspects. Unfortunately, this also includes instances of unprofessional behavior among colleagues and administration. This panel will address strategies for dealing with such behavior. What could/ should you do when you witness a young colleague being bullied by an older colleague who wields power at your institution? What can you do if you are on the receiving end of such behavior? How can you navigate institutional politics if you feel your ideas are unfairly shut down by your chair/dean? How can you improve a bad situation created by an uncooperative colleague in your department? How can you help foster healing and growth in your department following negative interpersonal dynamics? This topic is of concern to mathematicians at all stages of their career. Panelists are Amy Cohen-Corwin, Rutgers, The State University of New Jersey, Lloyd Douglas, Independent Consultant, Rick Gillman, Valparaiso University, and Paula Russo, Trinity College. This session is sponsored by Project NExT Peach Dots.

MAA Panel: Advising and Mentorship: Fostering Successful Students, organized by Ashley Johnson, University of North Alabama, and Alicia Prieto-Langarica, Youngstown State University; Thursday, 1:00-2:20 pm. As all faculty members know, there is more to teaching students than just what happens in a classroom two or three times per week. Some students look to faculty members for advice on which graduate programs to apply to, which conferences to attend, where to search for jobs, what kind of jobs to apply to, which summer programs to apply to, etc. Other students might not be aware of the opportunities that await them and it falls to the advisor to inform them. On top of the advice, we're very often asked to write letters of recommendation for all of these opportunities as well. The goal of this panel is to have experienced faculty members share their advising and mentorship experiences, as well as tips on successfully transitioning students into graduate school, summer programs, or the workforce, and writing effective letters of recommendation. Panelists are Jacqueline Jensen-Vallin, Lamar University, Hristo Kojouharov, University of Texas at Arlington, Marianne Korten, Kansas State University, Calandra Tate Moore, Department of Defense and Michael Young, Iowa State University.

MAA Panel: Pathways to Leadership, organized by Gretchen L. Matthews, Virginia Tech and Cynthia Curtis, The College of New Jersey; Thursday, 2:35-3:55 pm. This panel discusses a variety of pathways to leadership, addressing leadership in academic institutions at a variety of levels, in industry, and in professional societies. According to the AAUP report So Few Women Leaders, "women's paths to leadership often involve directing academic programs, chairing committees, or leading research centers or institutes that they initiate and for which they often 
obtain funding themselves." One finding demonstrates that traditional paths to leadership are slower and often blocked. The same is thought to be true for others from minorities underrepresented in STEM disciplines. The panel will discuss traditional routes through the welldefined hierarchy in academia along with alternative avenues mentioned above. Panelists are Jenna Carpenter, Campbell University, Barbara Faires, Westminster College, Susan Ganter, Embry-Riddle Aeronautical University, Kristin Lauter, Microsoft Research, Maura Mast, Fordham College at Rose Hill, Semra Skilic Bahi, Colby Sawyer College, and Judy Walker, University of Nebraska. This panel is sponsored by the MAA Committee on the Participation of Women in Mathematics.

MAA Panel: Advanced Placement Calculus and Student Understanding, organized by Gail Burrill, Michigan State University; Friday, 8:00-9:20 am. Each spring thousands of high school students take part in the Advanced Placement Calculus $\mathrm{AB}$ and $\mathrm{BC}$ examinations. This session will provide details on how the exams are graded and on student performance, in particular some of the key conceptual misunderstandings suggested in their responses to problems related to particluar calculus topics as well as areas in which they demonstrate competence. The panel will include representatives from the College Board, the college professor in charge of scoring these exams (aka, the Chief Reader), and some of the members of the Exam Development Committee who also serve as graders for the exam. There will be time in the session for the panelists to answer questions from the audience. Questions posed to the audience will be to consider how the areas of misconceptions are similar to or different from those evidenced by undergraduates in intro college calculus courses and what are possible strategies to address these misconceptions. Panelists are Gail Burrill, Michigan State University, Stephen Davis, Davidson University, Brendan Murphy, John Bapst High School, and Stephanie Odgen, College Board.

MAA Panel: Increasing Diversity and Retention in STEM Through Math-Focused First-Year Seminars, organized by Laramie Paxton, Washington State University; Friday, 9:3510:55 am. It is often cited that students abandon or avoid STEM disciplines due to difficulties with mathematics. While many colleges and universities have implemented first-year seminars, research studies specifically addressing the ability of math-focused seminars to address these barriers are not common. This Q \& A session will bring together diverse panelists to discuss the ability of math-focused first-year seminars and related programming to increase student interest and confidence in STEM disciplines. A special focus will be on students from underrepresented groups, increasing STEM retention rates, and diversifying STEM enrollments by reaching students who are otherwise not traditionally entering STEM fields. Panelists are Carlos Castillo-Garsow, Eastern Washington University, Maria Fung, Worcester State University, Guadalupe Lozano, University of Arizona, Shahriar Shahriari, Pomona College, and Francis Su, Harvey Mudd College.
MAA Panel: MAA Instructional Practices Guide's Value for Your Department, organized by Linda Braddy, Tarrant County College-Northwest campus, Kevin Charlwood, Washburn University, Daniel Maki, Indiana University Bloomington, and Catherine Murphy, Purdue University Northwest; Friday, 1:00-2:20 pm. The MAA's recently published Instructional Practices Guide (IPG) https: //www .dropbox.com/s/42oiptp46i0g2w2/MAA_IP_Guide _V1-2.pdf?d1=0 contains a wealth of information of value to mathematical sciences departments. It includes sections on classroom practices, assessment practices, design practices, technology, and equity and inclusion. Each section is rich with information and examples. You as Chair and your faculty may have questions you would like answered about some or all of these sections in order to address your interests. Our panel of leadership and IPG writing team members will provide insights into the construction of the Guide as well as answer the questions of importance to you and your faculty. Panelists are Martha Abell, Georgia Southern University, Linda Braddy, Tarrant County College-Northwest campus, Rick Cleary, Babson College, Doug Ensley, Mathematical Association of America, and Lew Ludwig, Denison University.

MAA Panel: Advising Actuarial Science Students, organized by Kevin Charlwood, Washburn University, Michelle Guan, Indiana University Northwest, Steve Paris, Florida State University, Barry Smith, Lebanon Valley College, and Sue Staples, Texas Christian University; Friday, 5:00-7:00 pm. A panel session dedicated to the concerns of faculty at institutions with actuarial science programs, designed to keep participants abreast of SOA/CAS curriculum and exam series changes that impact actuarial students and actuarial programs.Panelists are Kevin Charlwood, Washburn University, Rick Gorvett, Casualty Actuary Society (CASACT ),Michelle Guan, Indiana University Northwest, Stuart Klugman, Society of Actuaries, Steve Paris, Florida State University, Barry Smith, Lebanon Valley College and Sue Staples, Texas Christian University. This panel is sponsored by the MAA Committee on Actuarial Science Education.

MAA Panel: Calculus before the Senior Year of High School: Issues and Options, organized by David Bressoud, Macalester College; Saturday, 9:00-10:20 am. In spring 2017, 144,000 students took an AP Calculus exam before their senior year of high school. Of these, 44,000 took the BC exam. What should these students go on to take while still in high school? What mathematics will be most useful for those interested in pursuing biology, engineering, statistics, or pure mathematics? And this push of the post-secondary mathematics curriculum into ever earlier grades creates a host of other issues including preparing high school faculty to teach these courses, determining appropriate placement when these students get to college, and creating college courses and supports that articulate with their high school experience. This panel will explore these issues and examine some of the options. Panelists are Colin Adams, Williams College, Stephanie Ogden, College Board, Alison Reddy, University of Illinois, Urbana-Champaign, and Dan Teague, North 
Carolina School of Science and Mathematics. Sponsored by the College Board-MAA Committee on Mutual Concern.

MAA Panel: Listening and Responding to Students' Thinking, from Elementary to Undergraduate Mathematics, organized by Brad Ballinger, Humboldt State University, Christina Eubanks-Turner, Loyola Marymount University, Yvonne Lai, University of Nebraska - Lincoln, Cody L. Patterson, University of Texas at San Antonio, Priya V. Prasad, University of Texas at San Antonio, and April Strom, Scottsdale Community College; Saturday, 10:30 -11:50 am. One of the great joys and challenges in orchestrating mathematical discussions with undergraduate students is finding ways for students to build on each other's thinking. In teacher education and professional development settings, discussions also give future and current teachers the chance to learn in ways similar to how they would like to teach. But what are the differences and similarities across educational levels in mathematics discussions? How can we use knowledge of these differences and similarities productively in our own teaching? Join our discussion session on eliciting, representing, and responding to students' thinking. Our panelists-who span K-12, community college, and undergraduate levels-will each showcase an example of building on students' thinking from their own teaching. We will then as a group analyze how these examples compare, and what this may mean for our own teaching. Panelists are Gail Burrill, Michigan State University, Ted Coe, Achieve, and Brian Katz, Augustana College. This panel is sponsored by COMET (MAA's Committee on the Mathematical Education of Teachers) and SIGMAA-MKT (Special Interest Group of the MAA on Mathematical Knowledge for Teaching).

\section{MAA Poster and Other Sessions}

MAA Hrabowski-Gates-Tapia-McBay Session, organized by Ricardo Cortez, Tulane University; Wednesday, 9:0010:20 am. The Hrabowski-Gates-Tapia-McBay Session is named after four influential scientists of color: (1) Freeman Hrabowski, President of the University of Maryland at Baltimore County; (2) James S. Gates, University of Maryland, College Park; (3) Richard Tapia, Rice University; and (4) Shirley McBay, President of Quality Education for Minorities (QEM). Through multiple mechanisms, these Sessions expect to facilitate and accelerate the participation of scientists in the building of sustainable communities of mathematicians and mathematical scientists. In particular, the intention is to systematically recruit, welcome, encourage, mentor, and support individuals from underrepresented groups in the USA. This year the session will consist of a lecture from 9:00-9:50 am given by Rodrigo Bañuelos, Purdue University, title to be annnounced, and a short panel discussion, Actions to increase the participation of underrepresented minority groups in mathematics, from 9:50-10:20 am. The 2019 panel will focus on Actions to increase the participation of minority groups in mathematics. Panelists will include Minerva Cordero, University of Texas at Arlington; Pamela Harris, Williams College, and Rodrigo Bañuelos, Purdue University.
Town Hall Meeting: Spectra: Identifying Workplace Best Practices for LGBTQ Mathematicians, organized by David Crombecque, University of Southern California, Ron Buckmire, Division of Undergraduate Education, NSF, Christopher Goff, University of the Pacific, Alexander Hoover, Tulane University, and Douglas Lind, University of Washington, Seattle; Wednesday, 4:30-5:50 pm. The goal of this Town Hall Meeting is to learn from the audience examples of actions mathematical sciences departments are taking now to support LGBTQ mathematicians as well as to solicit suggestions of things departments can do in the future. In particular, how can departments be more inclusive and welcoming to LGBTQ individuals (undergraduate and graduate students, postdocs, adjuncts, tenured and non-tenured faculty)? In the second part of the Meeting the audience will be asked to brainstorm and provide suggestions on how SPECTRA can contribute to make these best practices known to the mathematical sciences community and to help departments implement them. During the meeting the audience will split into groups focused on specific topics, such as: Supporting transgender mathematicians in the work place; LGBTQ Mathematicians balancing work choices with family responsibilities; best practices for recruitment and retention of LGBTQ faculty. Other discussion topics can be suggested by the audience during the first part of the meeting. Each group will include a facilitator who will also serve as scribe to record the discussion. This town hall meeting is being organized by Spectra, the Association for LGBTQ Mathematicians.

Estimathon!, organized by Andy Niedermaier, Jane Street Capital; Thursday, 10:00 am-12 noon. They're called Fermi problems...

- How heavy is the Eiffel Tower?

- How many prime numbers have distinct digits?

- How many calories would you be eating if you had "one of everything" at the Cheesecake Factory?

If you're looking for a mind-bending mixture of math and trivia, look no further! Jane Street Capital presents The Estimathon contest: Teams will have 30 minutes to work on 13 problems, ranging from totally trivial to positively Putnamesque. Can your team beat the all-time best score? The top teams will receive prizes!

We will run 2 contests. Feel free to show up to either one!

(Please show up 15 minutes before the start time of the contest you want to join.)

Our target schedule is as follows:

10:0 am Welcome, overview of rules and scoring.

10:15 am Estimathon contest \#1

11:00 am Estimathon contest \#2

This event is sponsored by Jane Street Capital and the MAA Committee on Undergraduate Students (CUS).

The Dolciani Award Lectures, organized by Herbert A. Medina, Loyola Marymount University, and Tina Straley; Thursday, 1:00-2:00 pm. The MAA's Mary P. Dolciani Award recognizes a pure or applied mathematician who is making a distinguished contribution to the mathematical education of K-16 students in the US or Canada. This 
session will feature two recent recipients who will give overviews of the work that resulted in their nomination and subsequent prize.

MAA Poster Session: Mathematical Outreach Programs, organized by Betsy Yanik, Emporia State University; Friday, 10:00 am-12 noon. This poster session is designed to highlight special programs which have been developed to encourage students to maintain an interest in and commitment to succeeding in mathematics. These programs might include such activities as after school clubs, weekend activities, one day conferences, mentoring opportunities, summer camps, etc. This poster session encompasses a wide variety of outreach efforts for a variety of age groups. For example, programs might be designed to reach out to underrepresented groups. The projects supported by MAA Tensor and Summa grants will find this an ideal venue in which to share the progress of their funded projects. Another possible type of outreach might involve mathematical enrichment programs. Other examples might include innovative programs to motivate undergraduates to study mathematics. We encourage everyone involved with offering mathematical outreach activities to consider submitting an abstract to the session organizer, Betsy Yanik, eyanik@emporia.edu.

MAA Poster Session: Recreational Mathematics: Puzzles, Card Tricks, Games, and Gambling, organized by Paul R. Coe, Dominican University, Darren Glass, Gettysburg College, and Robert Vallin, Lamar University; Friday, 10:00 am-12 noon. Puzzles, card tricks, board games, game shows, gambling, and sports provide an excellent laboratory for testing mathematical strategy, probability, and enumeration. The analysis of such diversions is fertile ground for the application of mathematical and statistical theory. Solutions to new problems as well as novel solutions to old problems are welcome. Submissions by undergraduates or examples of the use of the solutions of these problems in the undergraduate classroom are encouraged. This session is sponsored by the SIGMAA on Recreational Mathematics.

MAA Poster Session: Activities for Teaching Multivariable Thinking through Data Visualization in Introductory Statistics, organized by Gregg Harbaugh, Boston University, and Soma Roy, California Polytechnic State University; Friday, 1:30-3:30 pm. The recently revised Guidelines for Assessment and Instruction in Statistics Education (GAISE) encourages statistics educators to teach multivariable thinking in introductory statistics classes, and a step towards this goal is to help students create and interpret graphical displays of data comprised of more than two variables. We invite proposals for poster presentations that showcase activities, datasets, and/or contexts that statistics teachers can use to motivate multivariable statistical thinking through data visualization and interpretation. Presentations should be based on activities that have actually been used in class, and accompanied by some evidence of the effectiveness of the activities in improving student understanding and interpretation of graphs and multivariable statistical thinking. A focus on modern approaches to teaching from a simulation-based perspective and incorporating data science ideas into the course are particularly encouraged. This session is sponsored by the SIGMAA Stat Ed.

MAA Poster Session: Projects Supported by the NSF Division of Undergraduate Education, organized by Jon Scott, Montgomery College, jon.scott@montgomery college.edu; Friday, 2:00-4:00 pm. This session will feature principal investigators (PIs) presenting progress and outcomes from various NSF funded projects in the Division of Undergraduate Education. The poster session format will permit ample opportunity for attendees to engage in small group discussions with the PIs and to network with each other. Information about presenters and their projects will appear in the program.

MAA Student Poster Session, organized by Chasen Smith, Georgia Southern University, and Eric Ruggieri, College of the Holy Cross; Friday, 4:306:00 pm. This session features research done by undergraduate students. First-year graduate students are eligible to present if their research was completed while they were still undergraduates. Research by high school students can be accepted if the research was conducted under the supervision of a faculty member at a postsecondary institution.

Appropriate content for a poster includes, but is not limited to, a new result, a new proof of a known result, a new mathematical model, an innovative solution to a Putnam problem, or a method of solution to an applied problem. Purely expository material is not appropriate for this session.

Participants should submit an abstract describing their research in 250 words or less by midnight, Friday, October 5,2018 . Notification of acceptance or rejection will be sent in early November. See www. maa.org/programs /students/undergraduate-research/jmm-student -poster-session for further information on what should be included in the abstract and a link to the abstract submission form.

Posters will be judged during the session, and certificates will be sent to presenters afterwards. Trifold, self-standing 48 " by 36 " tabletop poster boards will be provided. Additional materials and equipment are the responsibility of the presenters. Participants must set up posters between 2:30 and 3:30 pm and must be available at their posters from 3:30 to 6:00 pm. Judging will begin at $3: 30 \mathrm{pm}$ and general viewing will begin at 4:30 pm. Judges' feedback will be available at the MAA Pavilion in the Exhibit Hall on Saturday. Questions regarding this session should be directed to Eric Ruggieri eruggi er@holycross . edu and Chasen Smith csmi th@georgi asouthern. edu . This session is sponsored by the MAA Committee on Undergraduate Students (CUS).

Mathematically Bent Theater, featuring Colin Adams and The Mobiusbandaid Players; Friday, 6:007:00 pm. Which is funnier, homology or cohomology? Are there math humor receptors located in the brain and if not, why do we find math so funny? Did you key my car 
at Mathfest last summer? These are just a few of the questions we will not answer during this theatrical presentation of several short humorous mathematically inclined pieces.

Poetry Reading, organized by JoAnne Growney, Gizem Karaali, Lawrence M. Lesser, and Doug Norton; Friday, 7:00-8:30 pm. "Math poets, come help us resume our recent tradition of eclectic JMM poetry readings!" In 2019, we hope to especially feature poetry about math people-famous or not-including teachers, students, math-anxious characters, etc. All interested in mathematical poetry/art are welcome-come to share your poetry or simply enjoy the evening's offerings! Though we do not discourage last-minute decisions to participate, we encourage poets to submit poetry (no more than 3 poems, no longer than 5 minutes) and a 40-word bio in advance so that those selected can be listed in our printed program. Submit submissions (by November 1, 2018) or inquiries to Gizem Karaali (gizem. karaa7i@apomona. edu). This event is sponsored by Journal of Humanistic Mathematics and SIGMAA ARTS.

Backgammon! organized by Arthur Benjamin, Harvey Mudd College; Friday, 8:00-10:00 pm. Learn to play backgammon from expert players. It's a fun and exciting game where players with a good mathematics background have a decisive advantage. Boards and free lessons will be provided by members of the US Backgammon Federation. Stop by anytime!

Interactive Lecture for Students and Teachers, Ben Orlin, Math and Bad Drawings, Tic-Tac-Toe (or, What is Mathematics), Saturday, 10:00-10:50 am.

Math Circle Demonstration, organized by Lance Bryant, Shippensburg University, Sarah Bryant, Dickinson College, and Thomas J. Clark, Dordt College; Saturday morning. A math circle is an enrichment experience that brings mathematics professionals in direct contact with pre-college students and/or their teachers. Circles foster passion and excitement for deep mathematics. This demonstration session offers the opportunity for conference attendees to observe and then discuss a math circle experience designed for local students. While students are engaged in a mathematical investigation, mathematicians will have a discussion focused on appreciating and better understanding the organic and creative process of learning that circles offer, and on the logistics and dynamics of running an effective circle. The sponsor for this demonstration is SIGMAA MCST.

Math Wrangle, organized by Ed Keppelmann, University of Nevada-Reno, and Phil Yasskin, Texas A\&M University, Saturday afternoon. The Math Wrangle will pit teams of students against each other, the clock, and a slate of great math problems. The format of a Math Wrangle is designed to engage students in mathematical problem solving, promote effective teamwork, provide a venue for oral presentations, and develop critical listening skills. A Math Wrangle incorporates elements of team sports and debate, with a dose of strategy tossed in for good measure. The intention of the Math Wrangle demonstration at the Joint Math Meetings is to show how teachers, schools, circles, and clubs can get students started in this exciting combination of mathematical problem solving with careful argumentation via public speaking, strategy and rebuttal. ponsors for this event is SIGMAA for Math Circles for Students and Teachers (SIGMAA-MCST).

\section{Special Interest Groups of the MAA (SIGMAAs)}

SIGMAAs will be hosting a number of activities, sessions, and guest lectures. There are currently fourteen such focus groups in the MAA offering members opportunities to interact, not only at meetings, but throughout the year, via newsletters and email-based communications. For more information visit www. maa. org/community/sigmaas.

SIGMAA Officers Meeting, Thursday, 10:3012:00 noon, chaired by Andrew Miller, Belmont University.

SIGMAA on Mathematics and the Arts (SIGMAA ARTS) Mathematics and the Arts, Wednesday morning and afternoon (see MAA Contributed Paper Sessions).

Poetry Reading, Friday, 7:00-8:30 pm

SIGMAA on Business, Industry, and Government (BIG SIGMAA)

Mathematics Experiences and Projects in Business, Industry, and Government, Friday morning (see MAA Contributed Paper Sessions).

Guest Lecture, Friday, 4:30-5:15 pm.

Reception, Friday 5:15-6:00 pm.

Business Meeting, Thursday 9:00-10:00 am.

SIGMAA on Mathematical and Computational Biology (BIO SIGMAA)

Business Meeting and Reception, Friday, 6:007:00 pm.

Guest Lecture, Friday, 7:00-7:50 pm, Reinhard Laubenbacher, University of Connecticut, School of Medicine.

Mathematics \& the Life Sciences: Initiatives, Programs, Curricula, Thursday morning (see MAA Contributed Papers Section).

Trends in Mathematical and Computational Biology, Wednesday afternoon (see MAA Invited Paper Sessions).

SIGMAA on the History of Mathematics (HOM SIGMAA) Reception and Business Meeting, Wednesday, 6:157:15 pm.

Guest Lecture, Wednesday, 7:15-8:15 pm, Karen Parshall, University of Virginia, Crossing the Pond: European Mathematicians in 1920s America.

Ethnomathematics: Ideas \& Innovations in the Classroom, Friday morning (see MAA Contributed Papers Section).

SIGMAA on Inquiry Based Learning (SIGMAA IBL) Inquiry-Based Teaching and Learning, Friday morning and afternoon (see MAA Contributed Paper Sessions).

Business Meeting, Friday, 6:30-7:30 pm. 
MAA Minicourse: Introduction to IBL, Part A: Thursday 9:00-11:00 am and Part B: Saturday 9:00-11:00 am (see MAA Minicourses).

SIGMAA on Mathematical Knowledge for Teaching MAA Panel: Listening and Responding to Students' Thinking, from Elementary to Undergraduate Mathematics, Thursday afternoon (see MAA Panels).

SIGMAA on Math Circles for Students and Teachers (SIGMAA MCST)

MAA Panel: Listening and Responding to Students' Thinking, from Elementary to Undergraduate Mathematics, Friday, 8:00-9:20 am (see MAA Panels).

It's Circular: Conjecture, Compute, Iterate, Friday afternoon (see MAA Contributed Paper Sessions).

Math Circle Demonstration, Saturday.

Math Wrangle, Saturday.

MAA Minicourse: How to Run Successful Math Circles for Students and Teachers, Part A: Thursday 1:00-3:00 pm and Part B: Saturday 1:00-3:00 pm (see MAA Minicourses).

SIGMAA on the Philosophy of Mathematics (POM SIGMAA)

Reception, Thursday, 5:30-6:00 pm.

Business Meeting, Thursday, 6:00-6:15 pm.

Guest Lecture, Thursday, 6:15-7:05 pm, Michele Friend, George Washington University.

Philosophy of Mathematics, Friday morning (see MAA Contributed Paper Sessions).

SIGMAA on Quantitative Literacy (SIGMAA QL)

Innovative Pathways to Quantitative Literacy, Saturday morning (see MAA Contributed Paper Sessions).

Joint Guest Lecture \& Reception, Thursday, 6:00-7:30 pm, Dave Kung, St. Mary's College of Maryland and Kira Hamman, Penn State University.

SIGMAA on Recreational Mathematics

MAA Poster Session: Recreational Mathematics: Puzzles, Card Tricks, Games, \& Gambling, Friday, 10:00 am-12 noon (See MAA Poster Sessions).

SIGMAA on Research in Undergraduate Mathematics Education (SIGMAA on RUME)

Research in Undergraduate Mathematics Education, Thursday morning and Friday morning (see MAA Contributed Paper Sessions).

Research in Undergraduate Mathematics Education: Highlights from the Annual SIGMAA on RUME Conference, Saturday morning (see MAA Invited Paper Sessions).

SIGMAA on Sports

Mathematics \& Sports, Wednesday afternoon (see MAA Contributed Paper Sessions).

SIGMAA on Statistics Education (SIGMAA Stat Ed)
MAA Minicourse: Teaching Statistics using R and RStudio, Part A: Wednesday 1:00-3:00 pm and Part B: Friday 1:00-3:00 pm (see MAA Minicourses).

MAA Minicourse: Keep Teaching Statistics using $R$ and RStudio, Part A: Thursday 1:00-3:00 pm and Part B: Saturday 1:00-3:00 pm (see MAA Minicourses).

Infusing Data Science \& Big Data into the Statistics, Wednesday afternoon (see MAA Contributed Paper Sessions).

Technology \& Resources in Statistics Education, Friday afternoon (see MAA Contributed Paper Sessions).

MAA Poster Session: Activities for Teaching Multivariable Thinking through Data Visualization in Introductory Statistics, Friday, 1:30-3:30 pm (See MAA Poster Sessions).

Business Meeting, Friday, 6:30-7:30 pm.

Guest Lecture, Friday, 7:30-8:30 pm.

SIGMAA on Mathematics Instruction Using the Web (WEB SIGMAA)

Business Meeting, Reception, and Guest Lecture, Friday, 6:00-7:30 pm, Paul Seeberger, Monroe Community College, CalcPlot3D to Create Dynamic Figures for OER Textbooks and to 3D Print Surfaces for Multivariable Calculus and Beyond

MAA Minicourse: Visualizing Multivariable Calculus \& Differential Equations using CalcPlot3D, Part A: Thursday 9:00-11:00 am and Part B: Saturday, 9:00-11:00 am (see MAA Minicourses).

\section{MAA Sessions for Students}

Radical Dash! organized by Stacey Muir, University of Scranton, and Janine Janoski, Kings College; Radical Dash Kickoff Meeting: Wednesday, 10:20-10:50 am and Radical Dash Prize Session: Friday, 10:30-11:00 am. The Radical Dash is a multi-day scavenger hunt for teams of undergraduates filled with math challenges and creative activities. Clues will be released periodically via Instagram (follow us now@MAARadicalDash) tasking teams with doing things such as solving math problems, finding mathematical objects in everyday life, and hunting down locations throughout the conference. Team posts will be judged based on completion of tasks as well as creativity. Join us for the Radical Dash Kickoff on Wednesday, January $16,10: 20-10: 50$ am where team sign ups take place and more details will be provided. Individuals are welcome and encouraged to participate; they will be formed into teams on site at our Kickoff. Winners and prizes will be announced at the Radical Dash Prize Session on Friday, January 18, 10:30-11:00 am Questions? Email us at MAARadica1Dash@gmai1.com. Can't make the Kickoff? Email us by Tuesday, January 15. The Radical Dash! is sponsored by MAA Committee on Undergraduate Student Activities and Sections (CUSAC).

MAA Panel: What Every Student Should Know about the JMM, organized by Peri Shereen, California State University, Monterey Bay, Violeta Vasilevska, Utah Valley University; Wednesday, 9:00-10:20 am. Navigating a 
large conference can be overwhelming, even for those who have previously attended such an event. Panelists Joyati Debnath, Winona State University; Matt DeLong, Marian University, and Zsuzsanna Szaniszlo, Valparaiso University, will provide guidance for students attending the Joint Mathematics Meetings, including answers to some common questions: How do I get the most out of the program? What sessions are especially for students? What other events should I be on the lookout for? Will I understand any of the invited addresses or should I not bother attending them? If I am presenting a poster, where do I go to set it up? How can I get some cool, free math stuff? Students and their faculty mentors are encouraged to attend. Panelists are: Joyati Debnath, Winona State University, Matt DeLong, Marian University, and Zsuzsanna Szaniszlo, Valparaiso University. This panel is sponsored by the MAA Committee for Undergraduate Student Activities and Chapters (CUSAC).

Estimathon!, organized by Andy Niedermaier, Jane Street Capital; Thursday, 10:00 am-12 noon. They're called Fermi problems...

- How heavy is the Eiffel Tower?

- How many prime numbers have distinct digits?

- How many calories would you be eating if you had "one of everything" at the Cheesecake Factory?

If you're looking for a mindbending mixture of math and trivia, look no further! Jane Street Capital presents The Estimathon contest: teams will have 30 minutes to work on 13 problems, ranging from totally trivial to positively Putnamesque. Can your team beat the all-time best score? The top teams will receive prizes!

We will run 2 contests. Feel free to show up to either one!

(Please show up 15 minutes before the start time of the contest you want to join.)

Our target schedule is as follows:

10:0 am Welcome, overview of rules and scoring.

10:15 am Estimathon contest \#1

11:00 am Estimathon contest \#2

This event is sponsored by Jane Street Capital and the MAA Committee on Undergraduate Students (CUS).

Grad School Fair, Friday, 8:30-10:30 am. Here is the opportunity for undergrads to meet representatives from mathematical sciences graduate programs from universities all over the country. January is a great time for juniors to learn more, and college seniors may still be able to refine their search. This is your chance for one-stop shopping in the graduate school market. At last year's meeting over 300 students met with representatives from more than 60 graduate programs. If your school has a graduate program and you are interested in participating, for US\$125 a table will be provided for your posters and printed materials (registration for this event must be made by a person already registered for the JMM), and you are welcome to personally speak to interested students. Complimentary coffee will be served. Co-sponsored by the AMS and MAA.
MAA Lecture for Students, will be given by Annalisa Crannell, Franklin \& Marshall College, Drawing conclusions from drawing a square, Friday, 1:00 pm.

MAA Student Poster Session, organized by Chasen Smith, Georgia Southern University, and Eric Ruggieri, College of the Holy Cross; Friday, 4:306:00 pm. This session features research done by undergraduate students. First-year graduate students are eligible to present if their research was completed while they were still undergraduates. Research by high school students can be accepted if the research was conducted under the supervision of a faculty member at a postsecondary institution.

Appropriate content for a poster includes, but is not limited to, a new result, a new proof of a known result, a new mathematical model, an innovative solution to a Putnam problem, or a method of solution to an applied problem. Purely expository material is not appropriate for this session.

Participants should submit an abstract describing their research in 250 words or less by midnight, Friday, October 5,2018 . Notification of acceptance or rejection will be sent in early November. See www.maa.org/programs /students/undergraduate-research/jmm-student -poster-session for further information on what should be included in the abstract and a link to the abstract submission form.

Posters will be judged during the session, and certificates will be sent to presenters afterwards. Trifold, self-standing 48 " by 36 " tabletop poster boards will be provided. Additional materials and equipment are the responsibility of the presenters. Participants must set up posters between 2:30 and 3:30 pm and must be available at their posters from 3:30 to 6:00 pm. Judging will begin at $3: 30 \mathrm{pm}$, and general viewing will begin at 4:30 pm. Judges' feedback will be available at the MAA Pavilion in the Exhibit Hall on Saturday. Questions regarding this session should be directed to Eric Ruggieri eruggi er@holycross . edu and Chasen Smith csmi th@georgi asouthern. edu . This session is sponsored by the MAA Committee on Undergraduate Students (CUS).

MAA Interactive Lecture for Students and Teachers, will be given by Ben Orlin, Math and Bad Drawings, TicTac-Toe (or, What is Mathematics?), Saturday, 10:00-10:50 am,

\section{Project NExT}

Project NExT Workshop, Wednesday-Saturday, 8:00-6:00 pm.

Project NExT Lecture on Teaching, will be given by David Bressoud, Macalester College, Reflections on teaching calculus for the first time, 45 times, Thursday, 11:10-12 noon. 
See details about the reception on Friday in Social Events.

\section{Other MAA Events}

MAA Section Officers Meeting, Wednesday, 4:00-5:00 pm, chaired by Lisa Marano, Westchester University of Pennsylvania. Section officers will meet with members of the Committee on Sections and MAA staff to share information and discuss current initiatives.

SIGMAA Officers Meeting, Thursday, 10:30-12:00 noon, chaired by Andrew Miller, Belmont University.

MAA Business Meeting, Saturday, 11:10-11:40 am, chaired by MAA President Deanna Hausperger, Carleton College, and organized by MAA Secretary James Sellers, Pennsylvania State University.

\section{MAA Workshops}

MAA Workshop: NSF Funding Opportunities in the Education and Human Resources Directorate and the Division of Mathematical Sciences, organized by Karen Allen Keene, NSF, Division of Research on Learning; Wednesday, 9:35-10:55 am. A number of NSF divisions offer a variety of grant programs that promote innovations in learning and teaching and/or infrastructural support in the mathematical sciences. Following a short presentation about these programs (15 minutes), the remainder of the session will feature opportunities to engage in small group discussions with NSF staff about program features, current NSF policy changes, proposal preparation guidance, and other related topics. Presenters are Karen Allen Keene, Sandra Richardson, Talitha Washington, and Lee Zia, NSF, Division of Undergraduate Education, Karen King, NSF, Division of Research on Learning, Tara Smith, NSF, Division of Graduate Education, and Matt Douglass and Swatee Naik, NSF, Division of Mathematical Sciences. This workshop is sponsored by the MAA Subcommittee on Professional Development.

MAA Workshop: Discussing Project Ideas with NSF/ EHR Program Officers, Parts I and II, organized by Karen Allen Keene, NSF, Division of Research on Learning; Part I: Wednesday, 2:15-3:25 pm and Part II: Friday, 9:45-10:55 am. NSF program officers in the Directorate for Education and Human Resources will be available to talk with prospective Principal Investigators about potential project ideas. Following a very brief (5 minute) overview of NSF, participants may sign up for short (10 minute) time slots to speak one-on-one with program officers about the specifics of their ideas. (Participants are encouraged to bring a one-page description.) If you believe you have an idea, project or program worthy of NSF support that will positively impact pre-K-12, undergraduate, or graduate education in the mathematical sciences you should attend one of these two sessions. Presenters are Karen King, NSF, Division of Research on Learning, Tara Smith, NSF, Division of Graduate Education, and Karen Allen Keene, Sandra Richardson, Talitha Washington, and Lee Zia, NSF, Division of Undergraduate Education. This workshop is sponsored by the MAA Subcommittee on Professional Development.
MAA Workshop: Making it Happen: Modeling in Your Differential Equations Course, organized by Brian Winkel, SIMIODE; Thursday, 9:00-10:20 am. Workshop participants experience modeling with differential equations in a way they can bring into their own classroom. Modeling activities are used so teachers can experience, as students, what it is like to learn and teach in a modelingbased differential equations environment. We do this with engaging examples, situations in which modeling gives rise to mathematics. Modeling Scenarios from the SIMIODE--Systemic Initiative for Modeling Investigations and Opportunities with Differential Equations community at www. simi ode . org will serve as example opportunities. Participants generate and collect data through experiments, build a mathematical model, estimate parameters, validate the model, and create the need for learning about differential equations as a direct result of the modeling activity. Examples will involve collecting data and using data from sources, modeling, and parameter estimation. The demonstrations of the effective use of modeling to motivate the study of differential equations will be suitable for different school settings, high schools, two-year colleges, and four-year institutions. Workshop participants will leave with a large collection of materials they can use to offer a modeling-based approach in their own differential equations courses. Presenters are Audrey Malagon, West Virginia University, Rachel Rossetti, Agnes Scott University, Brian Winkel, SIMIODE, and Dina Yagodich, Frederick University.

MAA Workshop: For Faculty on Fostering Student Engagement: Experience Classroom Practices from the MAA IP Guide, organized by Carolyn Yackel, Mercer University; Thursday, 10:35-11:55 am. This workshop will help faculty gain experience with classroom practices promoted in MAA's new Instructional Practices (IP) Guide, focused on fostering student engagement and selecting appropriate mathematical tasks to facilitate active learning. Strom and Alvarez, the lead writers of the Classroom Practices section of the IP guide, will involve participants in investigating two techniques: (1) paired board work and (2) engaging students in mathematical critique and justification. First, participants will experience these techniques as students in the leaders' classroom, after which they will collectively debrief the experience from instructors' standpoints. Presenters are April Strom, Scottsdale Community College and James Alvarez, University of Texas at Arlington. This session is sponsored by the MAA Committee on the Teaching of Undergraduate Mathematics.

MAA Workshop: Calculus: Near-Numbers, organized by Frank Swenton, Middlebury College; Saturday, 9:45$10: 55 \mathrm{am}$. What is infinity? Why isn't zero times infinity zero? Why is infinity minus infinity indeterminate? A large proportion of student misapprehension of the core concepts of calculus (limits, convergence, divergence, etc.) derives from a single cause: that the classical (and outdated) mathematical language we use to talk about these concepts doesn't allow us to express them accurately and fully, particularly when it comes to their more subtle or troublesome (and often interesting) aspects. In this work- 
shop, aimed at both inst ructors and students of calculus, we'll preview and demonstrate videos and interactive instructional tools, freely available online, that can be used to shed new light on convergence, divergence, and limits by accurately animating these concepts and providing a language for teachers and students that allows them to say precisely what they mean to. In the process, we'll see straightforward, direct answers both to the sample questions above and to as many participant-suggested questions as time permits. The presenter is Frank Swenton, Middlebury College.

MAA Workshop: How to Talk about Math So People Want to Listen, organized by Paul Zorn, St. Olaf College; Thursday, 2:35-3:55 pm. Join podcast host, director and science writer Flora Lichtman and avid math communicator Rachel Levy for a workshop to help you practice communicating about mathematics to the audience of your choice. Previous attendees of our workshops have successfully engaged the popular press about their research. In this workshop we will focus on communicating about the value of mathematics to the general public, policy-makers and administrators. Presenters are Flora Lichtman, Gimlet Media, and Rachel Levy, Mathematical Association of America. This workshop is sponsored by the MAA Science Policy Committee.

\section{Activities of Other Organizations}

This section includes scientific sessions. Several organizations or special groups are having receptions or other social events. Please see the "Social Events" section of this announcement for those details.

\section{Association for Symbolic Logic (ASL)}

This two-day program on Friday and Saturday will include sessions of contributed papers as well as Invited Addresses by Douglas Cenzer, University of Florida, Natasha Dobrinen, University of Denver, Sergey Goncharov, Novosibirsk State University, John Krueger, University of North Texas, Michael C. Laskowski, University of Maryland, Jana Marikova, Western Illinois University and David Marker, University of Illinois at Chicago.

See also the sessions co-sponsored by the ASL in the “AMS Special Sessions" listings. These sessions include $D e$ finability and Decidability Problems in Number Theory on Thursday. Organizers for this session are Kirsten Eisenträger, Pennsylvania State University, Deidre Haskell, McMaster University, Jennifer Park, University of Michigan, and Alexandra Shlapentokh, East Carolina University. Also co-sponsored by the ASL is the session on Algorithmic Dimensions and Fractal Geometry on Wednesday. The organizers for this session are Jack H. Lutz, Iowa State University, and Elvira Mayordomo, University of Zaragoza, Spain.

\section{Association for Women in Mathematics (AWM)}

Fortieth Annual Noether Lecture, Thursday, 10:05 am, will be given by Bryna Kra, Northwestern University, $D y$ namics of systems with low complexity.
Association for Women in Mathematics Panel: Promoting Inclusion in STEM, organized by Talia Fernos, University of North Carolina Greensboro; Wednesday, 2:153:40 pm. The \#metoo movement has underscored the magnitude and prevalence of sexual harassment and how it impacts women's lives and careers. This topic is too often left out of conversations about attrition rates of women in STEM. In this panel discussion, we will examine what kinds of persistent issues contribute to underrepresentation in STEM, and brainstorm what measures can be taken to effectively overcome them.

Examples of underrepresented groups in STEM fields are cis and trans women, and gender minorities; racial and ethnic minorities, particularly people with African, Latin, and Native America descent; lesbian, gay, bisexual, transgender, and queer people; people coming from economically disadvantaged backgrounds; people with disabilities.

Attendees of the panel are expected to be respectful and sensitive to the issues faced by such individuals. Furthermore, because social stigmas and prejudice are major contributing factors to underrepresentation, attendees shall refrain from propagating stereotypes, or disparaging these groups. Everyone is encouraged to participate, independent of gender identity or expression, race, ethnicity, color, religion, age, national origin, sexual orientation, or disability.

This session is open to all JMM attendees. Panelists include Pamela Barnett, University of Pennsylvania. Harrison Bray, University of Michigan, Ann Arbor, Piper Harron, University of Hawaii, Manoa, Autumn Kent, University of Wisconsin, Madison, and other panelists to be announced. Talia Fernos, University of North Carolina Greensboro will be the panel moderator. https://sites .goog7e.com/site/awmpane12019/

Business Meeting, Wednesday, 3:45-4:15 pm. Chairs, Ami Radunskaya, AWM President and Ruth Hass, AWM President Elect.

Workshop Poster Presentations and Reception, Friday, 5:00-6:15 pm. AWM will conduct its workshop poster presentations by women graduate students. AWM seeks volunteers to serve as mentors for workshop participants. If you are interested, please contact the AWM office at awm@awm-math.org. This session is open to all JMM attendees.Organizers for these presentations are Sarah Witherspoon, Texas A\&M University, Liz Vivas, Ohio State University, and Matilde Lalin, Université de Montréal. The Posting Judging Coordinator is Emilie Wiesner, Ithaca College.

AWM Workshop: Special Session on WinCompTop: Applied and Computational Topology, Saturday, 8:00 am-5:00 pm, AWM will conduct its workshop with presentations by senior and junior women researchers. Updated information about the workshop is available at www. awm-math .org. All JMM attendees are invited to atend the program. Organizers for this workshop are Radmila Sazdanovic, North Carolina State University, and Yusu Wang, The Ohio State University.

Reception, Wednesday, 9:30-11:00 pm. See the listing in the Social Events, section of the announcement. 


\section{National Association of Mathematicians (NAM)}

Haynes-Granville-Browne Session of Presentations by Recent Doctoral Recipients in the Mathematical Sciences, Friday, 1:00-4:00 pm. Organized by Edray Goins, Purdue University/NAM.

Cox-Talbot Address, to be given Friday after the banquet by Talithia Williams, Harvey Mudd College, A Seat at the Table: Equity and Social Justice in Mathematics Education. See details about the banquet on Friday in the "Social Events" section.

Panel Discussion: NAM 2019-2069: Where Do We Go from Here?, Saturday, 9:00-9:50 am. The moderator for this panel will be Duane Cooper, Morehouse College. Panelists are Robert Bozeman, Morehouse College, Shea Burns, North Carolina A\&T State University, Robin Wilson, California State Polytechnic University, Pomona, and Shelby Wilson, Morehouse College.

Business Meeting, Saturday, 10:00-10:50 am.

Claytor-Woodward Lecture, Saturday, 1:00 pm, Henok Mawi, Howard University, On Mathematical Problems in Geometric Optics.

\section{National Science Foundation (NSF)}

The NSF will be represented at a booth in the exhibit area. NSF staff members will be available to provide counsel and information on NSF programs of interest to mathematicians. The booth is open the same days as the exhibits. Times that staff will be available will be posted at the booth. National Science Foundation (NSF)

MAA Workshop: NSF Funding Opportunities in the Education and Human Resources Directorate and the Division of Mathematical Sciences, organized by Karen Allen Keene, NSF, Division of Research on Learning; Wednesday, 9:35-10:55 am. (see MAA workshops).

AMS Special Event: Activities in NSF's Division of Mathematical Sciences (NSF-DMS), Wednesday, 2:15-4:30 pm. Organized by Henry Warchall, and Catherine Paolucci, National Science Foundation, Division of Mathematical Sciences. Come learn everything you always wanted to know about the National Science Foundation Division of Mathematical Sciences (DMS), which provides more than $60 \%$ of the federal support for basic mathematics research in the United States. What happens after my proposal is submitted? How much funding does DMS distribute, anyway? How is it allocated? What is this Merit Review Process of which you speak? How do I get involved? What funding programs can I apply to? What funding opportunities are available for students and postdocs?

DMS invites JMM 2019 attendees to get to know more about the Division's ongoing activities. This two-hour special event offers participants informal opportunities to meet and interact with DMS program directors as well as panel discussions on a variety of NSF-supported funding opportunities. It also provides updates on DMS events, such as the NSF-sponsored "We Are Mathematics" video contest. Undergraduate students, graduate students, and postdoctoral fellows are especially encouraged to attend.
MAA Workshop: Discussing Project Ideas with NSF/EHR Program Officers, Parts I and II, organized by Karen Allen Keene, NSF, Division of Research on Learning; Part I: Wednesday, 2:15-3:25 pm and Part II: Friday, 9:45-10:55 am. (see MAA workshops).

\section{Pi M Epsilon (PME)}

Council Meeting, Thursday, 8:00-11:00 am.

\section{Rocky Mountain Consortium}

Board Meeting, Friday, 2:15-4:00 pm.

\section{Society for Industrial and Applied Mathematics (SIAM)}

This program consists of an Invited Address, Development of Mathematical Methods for Next Generation Stent Design, at 11:10 am on Thursday given by Suncica Canic, University of California, Berkeley, and a series of Minisymposia to include Advances in mathematical modeling of complex materials systems, Maria Emilianenko, George Mason University; Mathematical Models in Cancer, Doron Levy, University of Maryland, College Park; Data Assimilation: Theory and Practice, John Harlim, Pennsylvania State University; Human Factors in Mathematics Education, Suzanne L. Weekes, Worcester Polytechnic Institute, Ron Buckmire, Occidental College, and Rachel Levy, Mathematical Association of America; Recent advances in mathematical theory and scientific computation for biological fluids, Suncica Canic, University of California, Berkeley; Recent Developments in numerical methods for fluids, Leo Rebholz, Clemson University; Analytical techniques in imaging electrical properties of tissue in coupled physics models, Alexandru Tamasan, University of Central Florida and Amir Moradifam, University of California, Riverside; Flow-Induced (In)Stability of Elastic Structures, Justin Webster, University of Maryland Baltimore.

See also the session co-sponsored by SIAM in the "AMS Special Sessions" listings AMS-MAA-SIAM Special Session on Research in Mathematics by Undergraduates and Students in Post-Baccalaureate Programs, organized by Darren A. Narayan, Rochester Institute of Technology, Khang Tran, California State University, Fresno, Mark David Ward, Purdue University, and John Wierman, The Johns Hopkins University.

\section{Other events}

Mathematical Art Exhibition, organized by Robert Fathauer, Tessellations Company; Nathan Selikoff, Digital Awakening Studios, and supported by the Special Interest Group of the MAA for Mathematics and the Arts, and the Bridges Organization. A popular feature at the Joint Mathematics Meetings, this exhibition provides a break in your day. On display are works in various media by artists who are inspired by mathematics and by mathematicians who use visual art to express their findings. Topology, fractals, polyhedra, and tiling are some of the ideas at play here. Do not miss this unique opportunity for a different 
perspective on mathematics. The exhibition will be located inside the Joint Mathematics Exhibits and open during the same exhibit hours.

Summer Program for Women in Mathematics (SPWM) Reunion, organized by Murli M. Gupta, George Washington University; Thursday, 1:00-3:00 pm. This is a reunion of the summer program participants from all 19 years (1995-2013) who are in various states of their mathematical careers: some are students and, others are in various jobs, both in academia as well as government and industry. The participants will describe their experiences relating to all aspects of their careers. There will also be a discussion on the increasing participation of women in mathematics over the past two decades and the national impact of SPWM and similar programs. See www. gwu . edu/ spwm.

\section{Mathemati-Con}

Events will take place on Saturday, January 19 between 9:00 am and 4:00 pm in a variety of locations at the JMM.

Some special presentations slated to be included in this program are the Who Wants to Be a Mathematician Championship, demonstrations of both Math Circles and Math Wrangles provided by the MAA SIGMAA on Math Circles for Students and Teachers, an Interactive Lecture for Teachers and Students presented by Ben Orlin, Math and Bad Drawings, the 2019 Mathematical Art Exhibition, a Showtime! presentation by the JPBM Communications Award recipient, and much more, concluding with the MAA-AMS-SIAM Gerald and Judith Porter Public Lecture, Big Data, Inequality, and Democracy, by Cathy O'Neil, CEO of ORCAA. All events on the Mathemati-Con program are open to the public.

Mathematical Art Exhibition, organized by Robert Fathauer, Tessellations Company; Nathan Selikoff, Digital Awakening Studios; and Elizabeth Whiteley, studio artist, Washington, DC, and supported by the Special Interest Group of the MAA for Mathematics and the Arts, and the Bridges Organization. A popular feature at the Joint Mathematics Meetings, this exhibition provides a break in your day. On display are works in various media by artists who are inspired by mathematics and by mathematicians who use visual art to express their love of mathematics. Topology, fractals, polyhedra, and tiling are some of the ideas at play here. Do not miss this unique opportunity for a different perspective on mathematics. The exhibition will be located inside the Joint Mathematics Exhibits and open during the same exhibit hours.

Interactive Lecture for Students and Teachers, Saturday,10:00-10:50 am, Ben Orlin, Math and Bad Drawings, Tic-Tac-Toe (or, What is Mathematics?).

Who Wants to Be a Mathematician Championship, organized by Michael A. Breen, American Mathematical Society, and William T. Butterworth, DePaul University; Saturday, 1:00 pm-2:45 pm. Show your support for top high school students from the US, Canada, and the UK in this international Who Wants to Be a Mathematician as they compete for a US $\$ 5,000$ first prize for themselves and US $\$ 5,000$ for their school's math department. Semifinals are at 1:00 pm and finals are at 2:00 pm. Come match wits with the contestants, support their mathematical achievement, and have tremendous fun at the same time.

Math Circle Demonstration, organized by Lance Bryant, Shippensburg University, Sarah Bryant, Dickinson College, and Thomas J. Clark, Dordt College; Saturday morning. A math circle is an enrichment experience that brings mathematics professionals in direct contact with pre-college students and/or their teachers. Circles foster passion and excitement for deep mathematics. This demonstration session offers the opportunity for conference attendees to observe and then discuss a math circle experience designed for local students. While students are engaged in a mathematical investigation, mathematicians will have a discussion focused on appreciating and better understanding the organic and creative process of learning that circles offer, and on the logistics and dynamics of running an effective circle. The sponsor for this demonstration is SIGMAA MCST.

Math Wrangle, organized by Ed Keppelmann, University of Nevada Reno, and Phil Yasskin, Texas A\&M University, Saturday afternoon. The Math Wrangle will pit teams of students against each other, the clock, and a slate of great math problems. The format of a Math Wrangle is designed to engage students in mathematical problem solving, promote effective teamwork, provide a venue for oral presentations, and develop critical listening skills. A Math Wrangle incorporates elements of team sports and debate, with a dose of strategy tossed in for good measure. The intention of the Math Wrangle demonstration at the Joint Math Meetings is to show how teachers, schools, circles, and clubs can get students started in this exciting combination of mathematical problem solving with careful argumentation via public speaking, strategy and rebuttal. Sponsors for this event is SIGMAA for Math Circles for Students and Teachers (SIGMAA-MCST).

Cathy O'Neil, CEO of ORCAA, Big data, inequality, and democracy, (MAA-AMS-SIAM Gerald and Judith Porter Public Lecture); Saturday, 3:00 pm.

\section{Welcoming Environment Policy}

The AMS and MAA strive to ensure that participants in the JMM enjoy a welcoming environment. In all its activities, the AMS and MAA seek to foster an atmosphere that encourages the free expression and exchange of ideas. The AMS and MAA support equality of opportunity and treatment for all participants, regardless of gender, gender identity or expression, race, color, national or ethnic origin, religion or religious belief, age, marital status, sexual orientation, disabilities, or veteran status.

Harassment is a form of misconduct that undermines the integrity of the AMS and MAA, and their activities and missions.

The AMS and MAA will make every effort to maintain an environment that is free of harassment, even though it does not control the behavior of third parties. A commitment to a welcoming environment is expected of all participants of JMM activities, including mathematicians, students, guests, staff, contractors and exhibitors, and participants in scientific sessions and social events. To 
this end, the AMS and MAA will include a statement concerning its expectations towards maintaining a welcoming environment in registration materials for the JMM, and has put in place a mechanism for reporting violations. Violations may be reported confidentially and anonymously to 855-282-5703 or at www.mathsociety.ethicspoint . com. The reporting mechanism ensures the respect of privacy while alerting the AMS and MAA to the situation. Violations may also be brought to the attention of the AMS Director of Meetings \& Conferences (who is usually at the meeting registration desk), and that person can provide advice about how to proceed.

\section{Exhibits}

The Joint Mathematics Meetings Exhibits include the country's leading scientific publishers, professional organizations, companies that offer mathematics-enrichment products and services, computer hardware and software companies, and the Mathematical Art Exhibit. It will be open to all registered participants on Wednesday (starting with the Grand Opening) 12:15 pm-5:30 pm, on Thursday and Friday 9:30 am-5:30 pm and on Saturday 9:00 amnoon. See more details at jointmathematicsmeetings. org.

AMS Book Sales and Membership: The American Mathematical Society booth, located in the Exhibit Hall, is where attendees will find the latest AMS titles (up to 40\% off list price), fun giveaways, mathematics awareness materials, and information about programs available to the mathematical community. Make sure to visit the booth to track your Mathematical Genealogy, learn about Mathematics Research Communities, or attend a demonstration of MathSciNet.

If you join the AMS or renew your membership during the meeting, you will receive a complimentary gift and will be able to start using your member discount on AMS titles right away. Make sure to ask about our new membership benefit, free shipping on all purchases, not just at meetings but also on orders placed online, phoned in, faxed, or sent via postal mail. Back by popular demand, the AMS Membership Department has once again arranged for a photographer to take AMS member's professional portraits. Visit amermathsoc.simplybook.me to schedule an appointment.

MAA Pavilion: Join us in the Mathematical Association of America (MAA) Pavilion, booths 424-431, for new networking events. It's the perfect opportunity to connect with the MAA and the mathematical community. You can also use this occasion to learn more about the MAA Career Resource Center, talk to staff about programs and resources for your research and classroom, renew your membership, and learn how MAA advances the understanding of mathematics, and its impact on our world.

Mathematical Art Exhibition, organized by Robert Fathauer, Tessellations Company; Nathan Selikoff, Digital
Awakening Studios; and Elizabeth Whiteley, studio artist, Washington, DC, and supported by the Special Interest Group of the MAA for Mathematics and the Arts, and the Bridges Organization. A popular feature at the Joint Mathematics Meetings, this exhibition provides a break in your day. On display are works in various media by artists who are inspired by mathematics and by mathematicians who use visual art to express their love of mathematics. Topology, fractals, polyhedra, and tiling are some of the ideas at play here. Do not miss this unique opportunity for a different perspective on mathematics. The exhibition will be located inside the Joint Mathematics Exhibits and open during the same exhibit hours.

\section{Hotel Accommodations}

The importance of reserving a hotel room at one of the official Joint Mathematics Meetings (JMM) hotels cannot be stressed enough. The AMS and the MAA make every effort to keep participants' expenses at the meeting, registration fees, and hotel rooms for the meeting as low as possible. They work hard to negotiate the most affordable hotel rates and to maximize the best use of your registration dollars. The AMS and MAA encourage all participants to register for the meeting. When anyone pays the registration fee and reserves a room with an official JMM hotel, he or she is helping to support not only the 2019 JMM, but future meetings as well.

General: Participants are encouraged to register for the JMM in order to reserve hotel rooms at the contracted JMM rates. If a participant needs to reserve a hotel room before they are registered for the JMM, he or she must contact the Mathematics Meetings Services Bureau (MMSB) at mmsb@ ams.org or 1-800-321-4267 ext. 4137 or ext. 4144 for further instructions.

Special rates have been negotiated exclusively for this meeting at the following hotels: Hilton Baltimore, Baltimore Marriott Inner Harbor, Sheraton Inner Harbor, Renaissance Harborplace Hotel, Hyatt Regency Baltimore, Royal Sonesta, Lord Baltimore Hotel, Hotel Monaco, Days Inn Inner Harbor, and Holiday Inn Inner Harbor. (See details on these hotels below.)

To receive the JMM rates, reservations for these hotels must be made through the MMSB. The hotels will not be able to accept reservations directly until after December 13, 2018. At that time, rooms and rates will be based on availability only. Any rooms reserved directly with the hotels after December 14, 2018 are subject to rates higher than the JMM rates.

A link to the 2019 JMM housing site will be included in the email confirmations of all registrations. If a participant needs to have the link emailed to him or her, please send the request to mmsb@ams.org. If anyone is having problems reserving a hotel room, please send email to mmsb@ams.org.

Any participant who needs to reserve a hotel room and does not have a credit card, he or she should contact the MMSB at mmsb@ams .org for further instructions. If a check is being used to reserve a hotel room, the reserva- 
tion and check must be received by the MMSB no later than December 1, 2018.

ADA Accessibility: We strive to take the appropriate steps required to ensure that no individual with a disability is excluded, denied services, segregated, or otherwise treated differently. If special assistance, auxiliary aids, or other reasonable accommodations to fully participate in this meeting is required, it should be indicated in the appropriate section on the Registration and Housing Form or emailed to the MMSB at mmsb@ams.org. Requests for ADA-accessible rooms should also be clearly indicated when making hotel reservations. All requests for special accommodations under the Americans with Disabilities Act of 1990 (ADA) must be made allowing enough time for evaluation and appropriate action by the AMS and MAA. Any information obtained about any disability will remain confidential.

\section{Cancellation Policies:}

- 24-hour cancellation policy: Sheraton Inner Harbor, Lord Baltimore Hotel, Days Inn Inner Harbor, and Holiday Inn Inner Harbor

- 48-hour cancellation policy: Baltimore Marriott Inner Harbor and Hotel Monaco

- 72-hour cancellation policy: Hilton Baltimore, Renaissance Harborplace Hotel, Hyatt Regency Baltimore, and Royal Sonesta

\section{Check-in/Checkout:}

- Check-in at 3:00 pm and checkout at 11:00 am: Days Inn Inner Harbor

- Check-in at 3:00 pm and checkout at noon: Hilton Baltimore, Sheraton Inner Harbor, Hotel Monaco, and Holiday Inn Inner Harbor

- Check-in at 4:00 pm and checkout at 11:00 am: Hyatt Regency Baltimore and Lord Baltimore Hotel

- Check-in at 4:00 pm and checkout at noon: Baltimore Marriott Inner Harbor, Renaissance Harborplace Hotel, and Royal Sonesta

Complimentary Room Drawing: Participants who register and reserve a hotel room by October 30, 2018, will be included in a lottery for complimentary hotel room nights during the meeting. Rooms with multiple occupants will be included. The winners will be notified by phone and/ or email prior to December 19, 2018.

Confirmations: An email confirmation number will be provided for each hotel reservation made online. This confirmation number will give participants direct access to edit their reservations, up to December 13, 2018. Those who did not receive a confirmation number or who have any questions about the reservation process should contact the MMSB at mmsb@ams.org or 1-800-321-4267, ext. 4137 or 4144 .

\section{Deadlines:}

- Chance to win complimentary hotel nights: October 30
- In time to have program mailed in December: November 20

- Hotel Reservation Changes, and Cancellations through the MMSB: December 13

Environmental Policies: All of the hotels listed have environmental-friendly programs in place.

Internet Access/Wireless: All of the hotels listed offer complimentary wireless internet in all public areas, the lobby, and all sleeping rooms.

Looking for a Roommate? An interactive search board is available for participants looking for a roommate. See bboards.jointmathematicsmeetings.org for more details.

Location: The Hilton Baltimore and the Baltimore Marriott Inner Harbor will be the co-headquarter hotels for this meeting. The JMM Registration Desk, exhibits, poster sessions, scientific sessions, and AMS Employment Center will be located in the Baltimore Convention Center. The AMS Short Course, MAA Minicourses, Committee meetings, social events and affiliate events will be held in the Hilton Baltimore, the Baltimore Marriott Inner Harbor, and the Baltimore Convention Center.

Hilton Baltimore (co-headquarter), 401 West Pratt Street Baltimore, MD 2120. Room rates are US\$179 for a single/ double room and US\$149 for a student rate single/double room. Smoke-free hotel; restaurants: Diamond Tavern, Lobby Bar, and The Coffee Bean \& Tea Leaf, fitness center; indoor pool; 24-hour business center available to registered guests; full amenities in guest rooms; laptop-sized safes in guest rooms; windows do not open; children under 12 are free in room with an adult; cribs available upon request at no charge; rollaways available only in king-bedded rooms; pets allowed; self-parking available for US\$33 per day with in/out privileges; valet parking available for US $\$ 43$ per day with in/out privileges; parking rates subject to change. The hotel does not offer an airport shuttle.

Baltimore Marriott Inner Harbor (co-headquarter), 110 South Eutaw Street, Baltimore, MD 21201. Room rates for this property are US\$169 single/double room and US $\$ 160$ for a student rate single/double room. Smoke-free hotel; restaurants: The Yard, BricknFire Pizza Company, Fresh Bites; fitness center; 24-hour business center available to registered guests; full amenities in guest rooms; laptopsized safes in guest rooms; windows do not open; children under 12 are free in room with an adult; cribs available upon request at no charge; rollaways available only in kingbedded rooms; no pets allowed; self-parking available for US\$30 per day with in/out privileges; parking rates subject to change. The hotel does not offer an airport shuttle.

Sheraton Inner Harbor, 300 South Charles Street Baltimore, MD 21201. Room rates at this property are US\$159 single/double room and US $\$ 145$ for a student rate single/ double room. Smoke-free hotel; restaurants: Orioles Grille 
Bar and Morton's The Steakhouse; fitness center; 24-hour business center available to registered guests; full amenities in guest rooms; laptop-sized safes in guest rooms; windows do not open; children under 12 free in room with an adult; cribs available upon request at no charge; rollaways available in king-bedded rooms only at no cost; dogs are allowed; valet parking available for US $\$ 42$ per day with in/out privileges; self-parking available for US\$28.50 per day with in/out privileges; parking rates subject to change. The hotel does not offer an airport shuttle.

Renaissance Harborplace Hotel, 202 East Pratt Street, Baltimore, MD 21201. Room rates are US $\$ 155$ for a single/ double room and US\$138 for a student rate single/double room. Smoke-free hotel; restaurants: Watertable, Watertable Lounge, and The Ground Floor Café \& Bar, Indoor pool; fitness center; 24-hour business center available to registered guests; full amenities in guest rooms; laptopsized safes in guest rooms; windows open in some rooms; children under 12 free in room with an adult; cribs available upon request at no charge; rollaways available in king-bedded rooms only for US\$25 per stay; no pets allowed; self-parking available for US\$33 per day with in/ out privileges; valet parking available for US $\$ 43$ per day with in/out privileges; parking rates subject to change. The hotel does not offer an airport shuttle.

Hyatt Regency Baltimore, 300 Light Street, Baltimore, MD 21201. Room rates are US\$150 for a single/double room and US\$140 for a student rate single/double room. Smoke-free hotel; restaurants: Bistro, Market, and Bistro 300 Lounge; fitness center; 24-hour business center available to registered guests; full amenities in guest rooms; laptop-sized safes in guest rooms; windows open in some rooms; children under 12 free in room with an adult; cribs available upon request at no charge; rollaways available in king-bedded rooms only at US\$25 per stay; pets are allowed; self-parking available for US\$30 per day with in/ out privileges; valet parking available for US $\$ 42$ per day with in/out privileges; parking rates subject to change. The hotel does not offer an airport shuttle.

Royal Sonesta, 550 Light Street Baltimore, MD 21201. Room rates are US\$149 for a single/double room and US\$134 for a student rate single/double room. Smokefree hotel; restaurants: Explorer's Restaurant and Formula Espresso; indoor pool; fitness center; 24-hour business center available to registered guests; full amenities in guest rooms; laptop-sized safes in guest rooms; windows open in suites only; children under 12 free in room with an adult; cribs available upon request at no charge; rollaways available in king-bedded rooms only for US $\$ 30$ per night; pets are allowed-one dog per room, up to 35 pounds with a US $\$ 75$ non-refundable deposit; self-parking available for US\$24 per day with in/out privileges; valet parking available for US\$39 per day with in/out privileges; parking rates subject to change. The hotel does not offer an airport shuttle.
Lord Baltimore Hotel, 20 West Baltimore Street Baltimore, MD 21201. Room rates are US\$139 for a single/double room and US $\$ 129$ for a student rate single/double room. Smoke-free hotel; restaurants: $L B$ Tavern and LB Bakery; fitness center; 24-hour business center available to registered guests; full amenities in guest rooms; laptop-sized safes in guest rooms; windows open in some rooms; children under 12 free in room with an adult; cribs available upon request at no charge; rollaways available in kingbedded rooms only for US\$20 per night; pets are allowed with a deposit of US\$50; self-parking available for US\$15 per day with in/out privileges; valet parking available for US\$31 per day with in/out privileges; parking rates subject to change. The hotel does not offer an airport shuttle.

Hotel Monaco, 2 North Charles Street, Baltimore, MD 21201. Room rates are US\$135 for a single/double and US\$125 for a student rate single/double room. Smokefree hotel; restaurant: $B \& O$ American Brasserie; fitness center; 24-hour business center available to registered guests; full amenities in guest rooms; laptop-sized safes in guest rooms; windows do not open; children under 12 free in room with an adult; cribs available upon request at no charge; rollaways available in king-bedded rooms only for US\$25 each; pets allowed-contact the hotel for specific costs, limitations, and instructions; valet parking available for US\$40 per day with in/out privileges; parking rates subject to change. The hotel does not offer an airport shuttle.

Days Inn Inner Harbor, 100 Hopkins Place, Baltimore, MD 21201. Room rates are US\$139 for a single/double room and US\$129 for a student rate single/double room. Smokefree hotel; restaurant: Harbor Grill and Lounge; fitness center; outdoor pool; 24-hour business center available to registered guests; full amenities in guest rooms; laptopsized safes in guest rooms; windows do not open; up to 2 children under 12 free in room with an adult, 3 or more children will be charged an additional fee; cribs available upon request at no charge; pets are allowed at US $\$ 100$ per stay; self-parking is available for US\$30 per day with in/ out privileges; parking rates subject to change. The hotel does not offer an airport shuttle.

Holiday Inn Inner Harbor, 301 West Lombard Street, Baltimore, MD 21201. Room rates are US\$139 for a single/ double room and US\$129 for a student rate single/double room. Smoke-free hotel; restaurants: Eden West Restaurant, Dottie's Café, and Pappas Sports Bar, fitness center; indoor heated pool; 24-hour business center available to registered guests; full amenities in guest rooms; safe deposit boxes available behind the front desk; windows do not open; children under 18 free in room with an adult; cribs available upon request at no charge; rollaways available in king-bedded rooms only; pets are allowed - US $\$ 50$ deposit per pet; self-parking available for US\$30 per day 
with in/out privileges; parking rates subject to change. The hotel does not offer an airport shuttle.

Parking: Please see the Parking section under "Travel" for any additional parking options. Parking information for each hotel is listed.

Rates: All rates are subject to applicable local and state taxes in effect at the time of check-in; currently 15.5\% state tax.

\section{Miscellaneous}

Audio-Visual Equipment: AMS Special Sessions and Contributed Papers, and MAA Invited and Contributed Paper Sessions, are provided with a screen and a LCD projector for projecting presentation slides. Blackboards, white boards, and transparency projectors are not available. Session rooms do not include an Internet connection or sound connection for videos or sound clips. For presentations using MAC computers, speakers are advised to bring the proper adaptors and equipment needed. LCD projectors are equipped with adapters to accept both VGA \& HDMI cables

Invited address talks (50-minutes long) are provided with a lectern, PC Laptop with Microsoft Office Suite including PowerPoint, wireless microphone, laser pointer, wireless slide advancer "clicker," document camera (for print materials and transparencies), and LCD projector for projecting presentation slides on large stage flanking screens. For presentations using MAC materials, speakers are advised to bring the proper adaptors and equipment needed. The Invited Address room does not include an Internet connection. Speakers that are planning to show videos should save them to their computers or USB drives prior to the meeting.

Overhead projectors are no longer provided as part of the standard audio-visual setup in any room. Any request for additional equipment should be sent to meet@ams . org and received by October 1 .

Child Care: The AMS and the MAA will provide a limited number of reimbursement grants of US $\$ 250$ per family to help with the cost of child care for registered participants at JMM 2019. The funds may be used for child care that frees a parent to participate more fully in JMM. Registration for the JMM as well as membership in the AMS or MAA is required to apply for this program.

Information about applying for child care grants will be available prior to the opening of advance registration in September; watch the JMM website for details. Applications will be accepted on a first-come, first-served basis until November 12, 2018. Final decisions on recipients will be made on or before November 28, 2018. All grant funds will provided in the form a check which will be issued at the JMM.

Email Services: Limited email access for all Joint Meetings participants will be available in an email center located adjacent to the JMM Registration Desk, in the Pratt St Lobby, on the 300 level of the Baltimore Convention Center. The hours of operation will be published in the program. Participants should be aware that complimen- tary wireless internet access will also be available in specific, designated areas of the Convention Center. These locations will be identified in the program.

Information Distribution: Tables are set up in the exhibit area for dissemination of general information of possible interest to the members and for the dissemination of information of a mathematical nature not promoting a product or program for sale. Information must be approved by the AMS Director of Meetings and Conferences prior to being placed on these tables.

If a person or group wishes to display information of a mathematical nature promoting a product or program for sale, they may do so in the exhibit area at the Joint Books, Journals, and Promotional Materials exhibit for a fee of US\$50 (posters are slightly higher) per item. Please contact the exhibits coordinator, MMSB, P.O. Box 6887, Providence, RI 02940, or by email at cpd@ams . org for further details.

The administration of these tables is in the hands of the AMS-MAA Joint Meetings Committee, as are all arrangements for Joint Mathematics Meetings.

Local Information: For information about the city, see https://baltimore.org/

Broadcasting, Photographing, and Videotaping Policy: Each invited address (50+minutes long) that takes place in the Invited Address room will be recorded, with the permission of the speaker, and posted online on a web page hosted by the Joint Mathematics Meetings. These recordings will be taken by a professional videographer hired by the Joint Mathematics Meetings.

The recording or broadcasting of any AMS or jointsponsored event, talk, and session by any other party is strictly forbidden without the explicit written permission of the AMS Executive Director or AMS Director of Meetings and Conferences. To obtain permission, send your request by email to meet@ams.org to the attention of the AMS Director of Meetings and Conferences. Having submitted a request form does not constitute temporary authority, and approval will not be given orally. Please allow sufficient time for the approval process to be completed. Allow at least two weeks from time of receipt of request by the AMS.

The recording or broadcasting of any MAA sponsored event by any other party is strictly forbidden without the explicit written permission of the Mathematical Association of America. To obtain permission to record and/or broadcast an MAA event or activity, complete the information requested in the Request to Record or Broadcast form on maa.org and send your request to the MAA Executive Director. You must have received a signed form granting approval for recording and/or broadcasting an MAA event before the event takes place. Having submitted a request form does not constitute temporary authority, and approval will not be given orally. Please allow sufficient time for the approval process to be completed. Allow at least two weeks from time of receipt of request by the MAA office.

Photographs and videos of meeting interactions will be taken by professional photographers hired by the Joint Mathematics Meetings or by AMS and MAA staff. These 
photographs and videos may occasionally be used for publicity purposes. By participating in the Joint Mathematics Meetings, participants acknowledge that their photograph or a video that includes them may be published in material produced by the Joint Mathematics Meetings, AMS or MAA. AMS and MAA are not responsible for unauthorized photographs, videos, or other images not taken by professional photographers hired by the Joint Mathematics Meetings or AMS and MAA staff.

Under no circumstances will anyone be permitted to take pictures or videos of a single person without that person's permission. Under no circumstances will anyone be permitted to take pictures or videos of an exhibitor's product or display without that exhibitor's permission.

Telephone Messages: It will be possible to leave a message for any registered participant at the meetings registration desk from January 16 through 19 during the hours that the desk is open. These messages will be posted on the Mathematics Meetings Message Board in the networking center; however, staff at the desk will try to locate a participant in the event of a bona fide emergency. The telephone number will be published in the program and daily newsletter.

\section{Registration}

The AMS and the MAA encourage all participants to register for the Joint Mathematics Meetings (JMM). The importance of registering for the meeting, especially before the meeting, cannot be overemphasized. Advanced registration fees are considerably lower than on-site registration fees and paying a registration fee helps to support a wide range of activities associated with planning, organizing, and executing the meetings.

All participants who wish to attend sessions are expected to register for the JMM and should be prepared to show their badges, if so requested. Badges are required to enter the Exhibits, the Employment Center, to obtain discounts at the AMS and MAA Book Sales, and to cash a check with the Joint Meetings cashier. The Mathematics Meetings Service Bureau (MMSB) is the official registration and housing bureau for the JMM and they will be processing all registrations.

Online Registration: To register and reserve a hotel room online, visit www.jointmathematicsmeetings.org /register. VISA, MasterCard, Discover, and American Express are the only methods of payment accepted for online registrations, and charges to credit cards will be made in US funds. Registration acknowledgments will be sent by email to the email addresses provided.

Paper Form Registration: For the convenience of those who do not have access to the internet or who prefer to not use the internet to register and reserve a hotel room, a paper copy of the registration form is located at the back of this issue. It is also located at www. jointmathematicsmeetings .org/meetings/national/jmm2019/JMM19_regform .pdf. If you are using this method to register for the meeting and do not have a credit card, please contact the MMSB at mmsb@ams . org for further instructions. If you are using a check to reserve your hotel room, your reservation and check must be received by the MMSB no later than December 1, 2018 to ensure that your check is validated and reaches the hotel in time to hold your room.

Forms must be mailed or faxed to the MMSB at MMSB, P.O. Box 6887, Providence, RI 02940 or 401-455-4004. For security reasons, credit card numbers by postal mail, email or fax cannot be accepted. If a participant is registering by paper form and would like to pay for his or her registration via credit card, he or she should indicate this on the form. MMSB staff will then contact that person.

NEW! Program Books: In order to keep registration fees as low as possible, save on postage, and make the JMM more environmentally friendly, program books will no longer be distributed or mailed in advance without your request. If you would like to receive a program book and pick it up at the meeting, please check the appropriate "YES" box on the Registration/Housing Form and pay a nominal fee of US\$5. If you would like to receive a program book and have it mailed to you before the meeting, please check the appropriate "YES" box on the Registration/Housing Form by November 20, 2018 and pay a nominal fee of US\$10. Programs can only be mailed via US Mail. Programs cannot be mailed to Canada, Mexico, or other countries outside of the US If you do not want to receive a program book (either before the meeting or at the meeting), please check the appropriate "NO" box on the Registration/Housing Form. Note that extra copies of the program book will be available on-site at the meeting for those that inadvertently checked the wrong box, while supplies last.

Updates and corrections received too late to be included in the program books will be included in the online program on the JMM website and in the JMM mobile app.

Badges and Tickets: As part of our efforts to keep registration fees as low as possible and save on postage, all other registration materials must be picked up at the meeting only. This includes badges, tickets, and promotional flyers. Badges will not be mailed before the meeting. All registration materials will be available at the Joint Mathematics Meetings Registration Desk, which will be located in the Pratt Street Lobby of the Baltimore Convention Center.

Participant Lists and Mailing Lists: To be included on the list of participants or the mailing list that is generated for the JMM, you must opt-in by checking the appropriate boxes on the Registration/Housing Form. All who do not opt-in on a category, will not be included in that category.

The list of participants will be included on the JMM daily newsletter, which is distributed during the meeting, and on the JMM mobile app. The mailing list will be used: a) by AMS and MAA staff to send marketing and promotional information by email, and b) by exhibitors and sponsors to send marketing and promotional information by US Mail. The AMS and MAA do not disseminate the email addresses of JMM participants to outside parties.

Cancellation Policy: Participants who cancel their registrations for the meetings, minicourses, short course, 
or banquet tickets by January $\mathbf{8 , 2 0 1 9}$, will be eligible to receive a $50 \%$ refund of fees paid. No refunds will be issued after this date.

Joint Mathematics Meetings Registration Fees Advanced At Meeting

(by Dec. 27)

Member of AMS, ASL, CMS, MAA, SIAM

Non-member US $\$ 345$ US $\$ 455$ 548 699

Graduate Student Member of AMS, ASL, CMS, MAA, SIAM 78

Graduate Student Non-member 124

Undergraduate Student Member of AMS, ASL, CMS, MAA, PME, KME, SIAM 78 137

Undergraduate Student Non-member 124

Temporarily Employed 281 322

Emeritus Member of AMS, MAA; Unemployed; High School Teacher; Developing Countries; Librarian High School Student

78 7 90

One-Day Member of AMS, ASL,

CMS, MAA, SIAM

One-Day Non-member

Non-mathematician Guest

Commercial Exhibitor

Art Exhibitor

MAA Minicourses

Grad School Fair Table

$\begin{array}{rr}\text { N/A } & 247 \\ \text { N/A } & 385 \\ 22 & 22 \\ 0 & 0 \\ 0 & 0 \\ 100 & 100 \\ 125 & 125\end{array}$

AMS Short Course:

Member of AMS

Non-member

124

190

Student/Unemployed/Emeritus should check with their department administrator to check their membership status.

Emeritus: Any person who has been a member of the AMS for twenty years or more and who retired because of age or long-term disability from his or her latest position is eligible for this category. Anyone person who has been a member of the MAA for 25 years and who is $70+$ years of age is also eligible for this category.

Librarian: Any librarian who is not a professional mathematician is eligible for this category.

Unemployed: Any person who is currently unemployed, actively seeking employment, and is not a student is eligible for this category. This category is not intended to include any person who has voluntarily resigned or retired from his or her latest position.

Developing Country Participant: Any person who is employed in developing countries where salary levels are radically not commensurate with those in the US is eligible for this category. See the most recent list of countries in this category at isge2018. isgesociety. com/registration /1ist-of-developing-countries.

Temporarily Employed: Any person currently employed but who will become unemployed by June 1, 2019, and who is actively seeking employment is eligible for this category.

Non-mathematician Guest: Any family member or friend, who is not a mathematician, and who is accompanied by a participant in the meetings is eligible for this category. Guests will receive a badge and may accompany a mathematician to a session or talk and enter the exhibit area.

Commercial Exhibitor: Any person who is exhibiting in the Joint Mathematics Meetings Exhibits is eligible for this category. This does not include anyone participating in a poster session. Any exhibitor who is a mathematician and is participating in the scientific program and/or wants to attend sessions, talks, etc. is expected to register separately for the meeting.

Art Exhibitor: Any person who is exhibiting in the Mathematical Art Exhibition is eligible for this category. This does not include anyone participating in a poster session. Any exhibitor who is a mathematician and is participating in the scientific program and/or wants to attend sessions, talks, etc. is expected to register separately for the meeting.

\section{Registration Deadlines}

There are three registration deadlines, each with its own benefits :

- ROOM LOTTERY (to qualify for hotel room lottery)October 30

- ORDINARY (to qualify to have program book mailed) - November 20

- FINAL (advanced registration, short course, minicourses, banquets)-December 27

Room Lottery: Participants who register by October 30 will be included in a random drawing to select winners of complimentary hotel room nights during the meeting. 
Rooms with multiple occupants will be included in the drawing. The location of these rooms will be based on the number of complimentary room nights earned in the various hotels. Therefore, a free room will not necessarily be in winner's first-choice hotel. All winners will be notified by phone and email prior to December 19.

Ordinary Registration: Participants who register by November 20 can choose to receive their materials before the meeting by mail. Badges will be distributed at the meeting only.

Final Registration: Participants who register after November 20 and by December 27 must pick up their badges, programs, and any tickets for social events at the meeting. After December 27, participants may register at the meeting, at higher fees.

\section{Social Events}

All events listed are open to all registered participants. It is strongly recommended that for any event requiring a ticket, tickets should be purchased through advance registration. Only a very limited number of tickets, if any, will be available for sale on site. If you must cancel your participation in a ticketed event, you may request a $50 \%$ refund by returning your tickets to the Mathematics Meetings Service Bureau (MMSB) by January 8, 2019. After that date, no refunds can be made. Special meals are available at banquets upon advance request, but this must be indicated on the Advanced Registration/Housing Form.

2019 AMS “Until Next Time” Social, Saturday, 7:009:30 pm. Join your colleagues on the last evening of JMM 2019 as we say "until next time!" This year's AMS closing event will be held on Saturday, January 19, 2019 from 7:00 pm-9:30 pm at the Maryland Science Center, 601 Light Street, Baltimore, MD, located in Baltimore's Inner Harbor just 0.6 miles away from the Baltimore Convention Center. Guests will enjoy the evening at this Baltimore destination with access to exhibits and hands-on displays. Join us for live music, various food stations, interactive activities and learning opportunities, and a chance to wish your colleagues well "until next time!"

While guests are welcome to choose their transportation method, we are pleased to provide a free shuttle service. Shuttle service will pick up guests every 10-15 minutes beginning at 6:45 pm from both the Hilton Baltimore and Baltimore Convention Center and dropping off at the venue. Return shuttle service will pick up guests at the Maryland Science Center beginning at 7:30 pm and return guests to the Baltimore Convention Center and Hilton Baltimore. Accessible shuttle service will be available.

Purchase your tickets when registering for the 2019 Joint Mathematics Meetings at: jointmathematicsmeetings . org. Regular Tickets: US\$75; Student Tickets: US\$35. A limited number of tickets will be available at the special students rate.

Association of Christians in the Mathematical Sciences (ACMS) Reception and Lecture, Thursday, 5:30-7:30 pm. The reception will take place between 5:30 and $6: 30 \mathrm{pm}$, followed by a short program and 20 minute talk at 6:30 pm. Students are encouraged to attend, and opportunity will be provided afterwards for delegates to go to dinner at a local restaurant. The talk will be given by Sloan Despeaux.

Association for Women in Mathematics Reception and Awards Presentation, the AWM Reception which is open to all JMM participants will be held on Wednesday at 9:30 pm after the AMS Gibbs Lecture. The AWM President at 10:00 pm will recognize all of the honorees of the AWM Alice T. Schafer Prize for Excellence in Mathematics by an Undergraduate Woman, the recipients of the AWM Dissertation Prize, the AWM Service Awards, and the AWM Fellows.

Backgammon! organized by Arthur Benjamin, Harvey Mudd College; Friday, 8:00-10:00 pm. Learn to play backgammon from expert players. It's a fun and exciting game where players with a good mathematics background have a decisive advantage. Boards and free lessons will be provided by members of the US Backgammon Federation. Stop by anytime!

Budapest Semesters in Mathematics Annual Alumni Reunion, Thursday, 5:30-6:30 pm.

BSME Information Session, Friday, 12:00-1:00 pm. BSME (Budapest Semesters in Mathematics Education) is a study abroad program in Budapest, Hungary, designed for undergraduates (and recent graduates) interested in teaching middle school or high school mathematics. Participants will study the Hungarian approach to learning and teaching, in which a strong and explicit emphasis is placed on problem solving, mathematical creativity, and communication. Come learn more about this exciting new program. bsmeducation .com

University of California, San Diego Reception, Thursday, 6:00-8:00 pm. Reception for Mathematics alumni.

Reception for Graduate Students and First-Time Participants, Wednesday, 5:30-6:30 pm. The AMS and MAA cosponsor this social hour. Graduate students and first-timers are especially encouraged to come and meet some old-timers to pick up a few tips on how to survive the environment of a large meeting. Light refreshments will be served.

ICERM Mixer, Friday, 6:00-8:00 pm. ICERM welcomes all past and future participants of our semester programs, workshops, collaborations and Summer@ICERM undergraduate programs to come together for its annual mixer Those interested in the Simons collaboration 'Arithmetic Geometry, Number Theory, and Computation' are also invited. Refreshments will be served.

University of Illinois at Urbana-Champaign, Friday, 6:00-7:30 pm. Department of Mathematics Reception. Everyone ever connected with the Department is encouraged to get together for conversation and to hear about mathematics at the University of Illinois.

Joint Prize Reception, Thursday 5:30-6:30 pm.

Knitting Circle, Thursday, 8:15-9:45 pm. Bring a project (knitting/crochet/tatting/beading/etc.) and chat with other mathematical crafters! 
MAA/Project NExT Reception, Friday, 8:00-10:00 pm. All Project NExT Fellows, consultants, and other friends of MAA Project NExT are invited. Organizers: Julia Barnes, West Carolina University, Alissa Crans, Loyola Marymount University, Matt DeLong, Taylor University and David Kung, St Mary's College of Maryland.

MAA Two-Year College Reception, Wednesday, 5:307:00 pm, is open to all meeting participants, particularly two-year faculty members. This is a great opportunity to meet old friends and make some new ones.

Mathematical Reviews Reception, Friday, 6:00-7:00 $\mathrm{pm}$. All friends of the Mathematical Reviews (MathSciNet) are invited to join reviewers and MR editors and staff (past and present) for a reception in honor of all of the efforts that go into the creation and publication of the Mathematical Reviews database. Refreshments will be served.

Mathematical Institutes Open House, Wednesday, 5:30-8:00 pm. Please join us at the Mathematical Institutes Open House reception to learn about the latest programs and workshops being held by a number of institutes. Hope to see you there.

The Mathematical Sciences Research Institute (MSRI) Reception for Current and Future Donors, Thursday, 6:30-8:00 pm. MSRI invites current and prospective donors to an informal reception with appetizers and drinks. Directors David Eisenbud and Hélène Barcelo will speak about present and upcoming events and programs, as well as the impact of private support on the Institute.

MSRI thanks and acknowledges mathematicians who support MSRI's programs and workshops through membership in the Archimedes Society or the Gauss Society. Archimedes Society members support MSRI with annual gifts. Gauss Society members support MSRI with planned gifts through arrangements in their wills and estates.

For more information about the event and how to join the Archimedes or Gauss Societies, please contact, Annie Averitt, Director for Advancement and External Relations, aaveritt@msri .org; 510-643-6056. www. msri .org.

\{MathILy, MathILy-Er\} Yearly Gather, Wednesday, 7:00-8:30 pm. Come one, come all, and play a recently created mathematical game or solve a new puzzle! And learn about the \{MathILy, MathILy-Er\} summer programs for high-school students, then hang out with \{MathILy, MathILy-Er\} alumnus and instructors.

University of Michigan Mathematics Alumni and Friends Reception, Thursday, 5:30-7:00 pm.

National Association of Mathematicians Banquet, Friday, 6:00-8:40 pm. A cash bar reception will be held at 6:00 pm, and dinner will be served at 6:30 pm. Tickets are US\$65 each, including tax and gratuity. The Cox-Talbot Invited Address will be given after the dinner.

NSA Women in Mathematics Society Networking Session, Thursday, 6:00-8:00 pm.

PROMYS and Ross Reception for Alumni and Friends, Thursday, 6:30-8:30 pm. There will be hors d'oeuvres, a cash bar, and interesting conversations!

SCUDEM Gathering and Reunion, Friday, 7:00-8:30 pm. SIMIODE is holding an information Gathering for SCUDEM
IV 2019 - Student Competition Using Differential Equations Modeling for those interested in learning about hosting and participating in this team event for high school and undergraduate students scheduled for October 2019. This will be an opportunitiy for past participants (local site host coordinators, coaches, and students) to reunite and share their experiences and for colleagues who want to learn more about SCUDEM.

Spectra Reception for LGBT Mathematicians and Allies, Thursday, 6:00-8:00 pm. Annual reception for lesbian, gay, bisexual, and transgender mathematicians and their allies. Begun in 1996, this event is organized by Spectra, a volunteer association for LGBT mathematicians that is affiliated with NOGLSTP, the National Organization of Gay and Lesbian Scientists and Technical Professionals.

University of Tennessee Mathematics Department Alumni and Friends Reception, Thursday, 5:30-7:30 pm. Alumni and other friends of the University of Tennessee Mathematics Department, including prospective graduate students, are welcome to come and enjoy snacks, renew old friendships and make new ones.

Texas A\&M University Mathematics Department Reception for Alumni, Students, and Faculty, Friday, 5:30-7:30 pm. All alumni, current students, faculty, and current and former post-docs are encouraged to join us for this reception.

Reception for Undergraduates, Wednesday, 4:30-5:30 pm.

University of Waterloo Faculty of Mathematics Reception, Thursday, 6:00-8:00 pm. Join the University of Waterloo Faculty of Mathematics for a cocktail reception celebrating the accomplishments of our Faculty, and its' members over the past year. All welcome, refreshments provided.

YP17HCSSiM Reunion Breakfast, Friday, 7:17-9:00 am.

\section{Travel/Transportation}

The 2019 Joint Mathematics Meetings will be held at the Baltimore Convention Center, located at 1 W. Pratt Street, Baltimore, MD 21201. Some committee meetings and social events will be held at the Hilton Baltimore and the Marriott Inner Harbor. Baltimore is on Eastern Standard Time.

The Baltimore/Washington International Thurgood Marshall Airport (BWI) (https: //www. bwi ai rport.com/) is served by all major airlines and is approximately 12 miles from the Inner Harbor area where the Baltimore Convention Center and the conference hotels are located. The street address of the airport is 7062 Friendship Rd, Baltimore, MD 21240.

\section{Airline}

The official airline for this meeting is Southwest Airlines. Beginning on August 15, Joint Mathematics Meetings participants will receive a discount and bonus Rapid Reward points from Southwest Airlines through Southwest's SWABIZ $^{\circledR}$ account. Southwest Airlines is offering an $8 \%$ discount off Anytime \& Business Select ${ }^{\circledR}$ fares and a $2 \%$ discount off select 'Wanna Get Away ${ }^{\circledR}$ ' fares for travel to 
and from the conference. Southwest is also offering no baggage fees for the first two bags, no change or cancellation fees, and no peak travel or fuel surcharges. Book your travel between August 15, 2018 and December 29, 2018 to take advantage of the discounted rates. Discounts are available for travel January 12, 2019 through January 22, 2019. To take advantage of this offer, follow these steps: (1) Go to www . swabiz . com (2) Click on 'Flight,' and (3) Enter Corporate ID: $\mathbf{9 9 8 8 4 5 4 0 .}$

If you are a member of the Southwest Rapid Rewards program, you will receive 50\% bonus Rapid Reward points for your travel to and from the meeting if you add your Rapid Rewards number to your reservation. To enroll in the Rapid Rewards program, visit www. southwest.com /corporaterapidrewards.

\section{Train}

Amtrak Baltimore Penn Station is located at 1515 North Charles Street, Baltimore, MD, 21201, approximately two miles from the Inner Harbor area. For information about rail service to Baltimore, please call 1-800-USA-RAIL, or visit www. amtrak. com.

\section{Ground Transportation}

Car Rental: The car rental facility at BWI is located at 7432 New Ridge Road, Hanover, MD, 21076. All of the major car rental agencies are available. Their free shuttle service carries customers to and from the airport approximately every 10 minutes. The shuttle leaves the lower level terminal near the baggage claim area. The trip takes around 10 minutes.

Hertz is the official car rental company for the meeting. To access the JMM special meeting rates at www. hertz . com, please click on the words "Discount Code" and enter CV\#04N30009 as the convention number. Reservations can also be made by calling Hertz directly at 800-654-2240 (U.S. and Canada) or 405-749-4434. Meeting rates include unlimited mileage and are subject to availability. Advance reservations are recommended and blackout dates may apply. Government surcharges, taxes, tax reimbursement, airport-related fees, vehicle licensing fees and optional items are extra. Standard rental conditions and qualifications apply. Minimum rental age is 20 (age differential charge for 20-24 applies). At the time of your reservation, the meeting rates will be automatically compared to other Hertz rates and you will be quoted the best comparable rate available.

Shuttles: The authorized list of shuttle and limousine companies at BWI can be found at https://www. bwiairport .com/to-from-bwi/transportation/authorized-pre -arranged-commercial-transportation-operators.

Taxi: The taxi stand at BWI is located just outside of the baggage claim area of the lower level of the terminal near doors 5 and 13. There are always taxis available. For more information, call 410-859-1100 or visit www. bwi ai rport taxi.com. The average taxi fare to the Baltimore Inner Harbor area is US\$30.

App-Based Ride Services: App-Based Ride Services such as Uber and Lyft pick up and drop off passengers at the terminal curbs at the Departures/Ticketing Level between doors 9 and 11.

Public Transportation: The Maryland Transit Administration (MTA) in Baltimore has a regular bus, subway, and light rail system. For details, maps, and schedules see mta. mary land.gov/ or call 410-539-5000. For particular inquiries, call 410-767-3999. One-way fares are currently US\$1.80. The Light Rail is recommended for travel from the airport to the Convention Center area via public transportation.

Light Rail from the Airport: Take the Light Rail from the BWI Marshall Light Rail Station, located outside the lower level of the terminal building near Concourse $\mathrm{E}$. The route is called "Hunt Valley and BWI Marshall Airport." Take the train towards Hunt Valley. There is a stop at the Convention Center (Howard and Pratt Street), after Camden Yards, and the Hilton and Marriott Inner Harbor hotels are one block west of the stop. For more details, see mta.maryland .gov/1 ight-rai 1. The train runs from 5:00 am to 11:00 pm weekdays, 6:00 am to 11:00 pm on Saturday, and 11:00 am to 7:00 pm on Sunday, approximately every 30 minutes (varies with time of day) and takes about 30-35 minutes. The cost is currently US $\$ 1.80$ one way.

Charm City Circulator: Baltimore has a free shuttle service with four routes around Baltimore, called the Charm City Circulator (CCC), see www. charmcitycirculator .com/ for information on routes and schedules. The Green Route runs from City Hall to Fells Point to Johns Hopkins, the Purple Route runs from 33rd Street to Federal Hill,the Orange Route runs from Hollins Market to Harbor East, and the Banner Route runs from the Inner Harbor to Fort McHenry. All the routes have stops in the Inner Harbor area.

The Orange route has a Convention Center stop, and the Banner Route has stops at Pratt Street and at Otterbein, both near the Convention Center. The Purple route has a stop at Light Street and E. Pratt Street, called Pratt Street-Inner Harbor. The Green Route can be picked up at. E. Baltimore Street and N. Gay Street. Many local attractions are accessible on the CCC. The National Aquarium and the Jewish Museum of Maryland are on the Orange Line. The Banner Line goes to the Maryland Science Center, Fort McHenry, the Baltimore Museum of Industry, and the American Visionary Art Museum. The Green Line travels near the Frederick Douglass-Isaac Myers Maritime Park Museum. The Charm City Circulator does not go to the airport.

Parking: There is a link to an interactive map of parking garages at the "Visit Baltimore" parking informa- 
tion page at https://baltimore.org/info/parking -information. This parking information page also offers a service called "Parking Panda," which allows you to book your parking space at a nearby garage in advance; see https://www. parkingpanda.com/ba1timore-parking.

Driving Directions from the airport to the Convention Center: Start out going southeast on Friendship Road toward Oak Road. Keep left to continue on Service Road, Lower Level, and then continue onto Friendship Road. Take I-195 W (follow signs for MD-170/Interstate 95/Annapolis/Baltimore/Washington). Merge onto I-95 N via exit 4A toward I-695/Baltimore. Take the I-395 N exit (Exit 53) toward Downtown/Inner Harbor. Merge onto I-395 N. Stay straight to go onto S. Howard Street, and take the first right onto W. Pratt Street. The Convention Center will be on your right (12.3 miles).
Will you be attending the Joint Mathematics Meetings in Baltimore, MD?

\section{AMS Members, have your professional portrait taken at the AMS Membership Booth!}

Back by popular demand! The AMS Membership Department has arranged for a photographer to take your professional portrait and have it emailed to you in just a few minutes. Consider uploading this photo to your MathSciNet ${ }^{\circledR}$ Author Profile page, using it on your university website, submitting it as the professional photograph for your book publication, or using it as your profile picture in email and on social platforms.

Availability:

THURSDAY, JAN. $17^{\text {TH }}$, 9:30AM-4:25PM

FRIDAY, JAN. 18'T $, 9: 30$ AM-4:25PM

\section{Schedule Your Appointment at: amermathsoc.simplybook.me}

Visit the AMS Membership Booth to learn more about the benefits of membership: In addition to receiving a discount on books purchased through the online bookstore and at meetings, members are also entitled to receive free shipping on their purchases, free and discounted subscriptions to journals, and access to colleagues via the Member Directory.

Join or renew your membership at JMM and receive a complimentary gift!

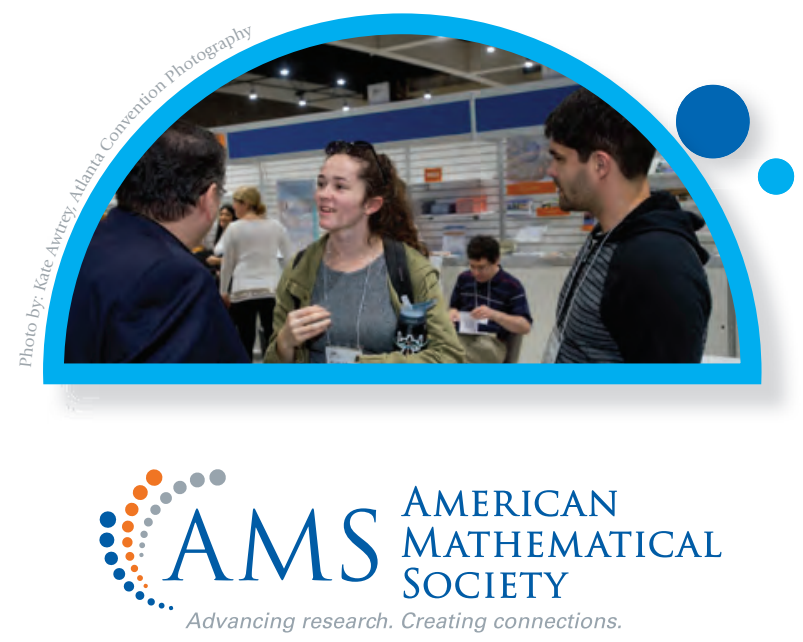




\section{JOINT MATHEMATICS MEETINGS}

\section{January 16-19, 2019 - Baltimore. Maryland}

HOTELS

1. Hilton Baltimore ( $\mathrm{CO}-\mathrm{HO})$ 401 W Pratt St. • Baltimore, MD 21201

2. Baltimore Marriott Inner Harbor at Camden Yards ( $\mathrm{CO}-\mathrm{HQ})$ 110 S Eutaw St. • Baltimore, MD 21202

3. Days Inn Inner Harbor 100 Hopkins Plaza · Baltimore, MD 21201

4. Holiday Inn Inner Harbor 301 W Lombard St. • Baltimore, MD 21202

5. Hotel Monaco

2 N Charles St. • Baltimore, MD 21201

6. Hyatt Regency Baltimore 300 Light St. • Baltimore, MD 21202

7. Lord Baltimore Hotel 20 W Baltimore St. • Baltimore, MD 21201

8. Renaissance Baltimore Harborplace 202 E Pratt St. • Baltimore, MD 21202

9. Royal Sonesta Harbor Court 550 Light St. • Baltimore, MD 21202

10. Sheraton Inner Harbor Hotel 300 S Charles St. • Baltimore, MD 21201

TRANSPORTATION

-M- Metro Line

$\mathbf{=}=$ Light Rail

MARC Train

-..... Water Taxies

(i) Visitors Center

$\longrightarrow$ Charm City Circulator - Green Route

$\because$ Charm City Circulator - Orange Route

$\longrightarrow$ Charm City Circulator - Purple Route

—- Charm City Circulator - Banner Route Parks and green spaces

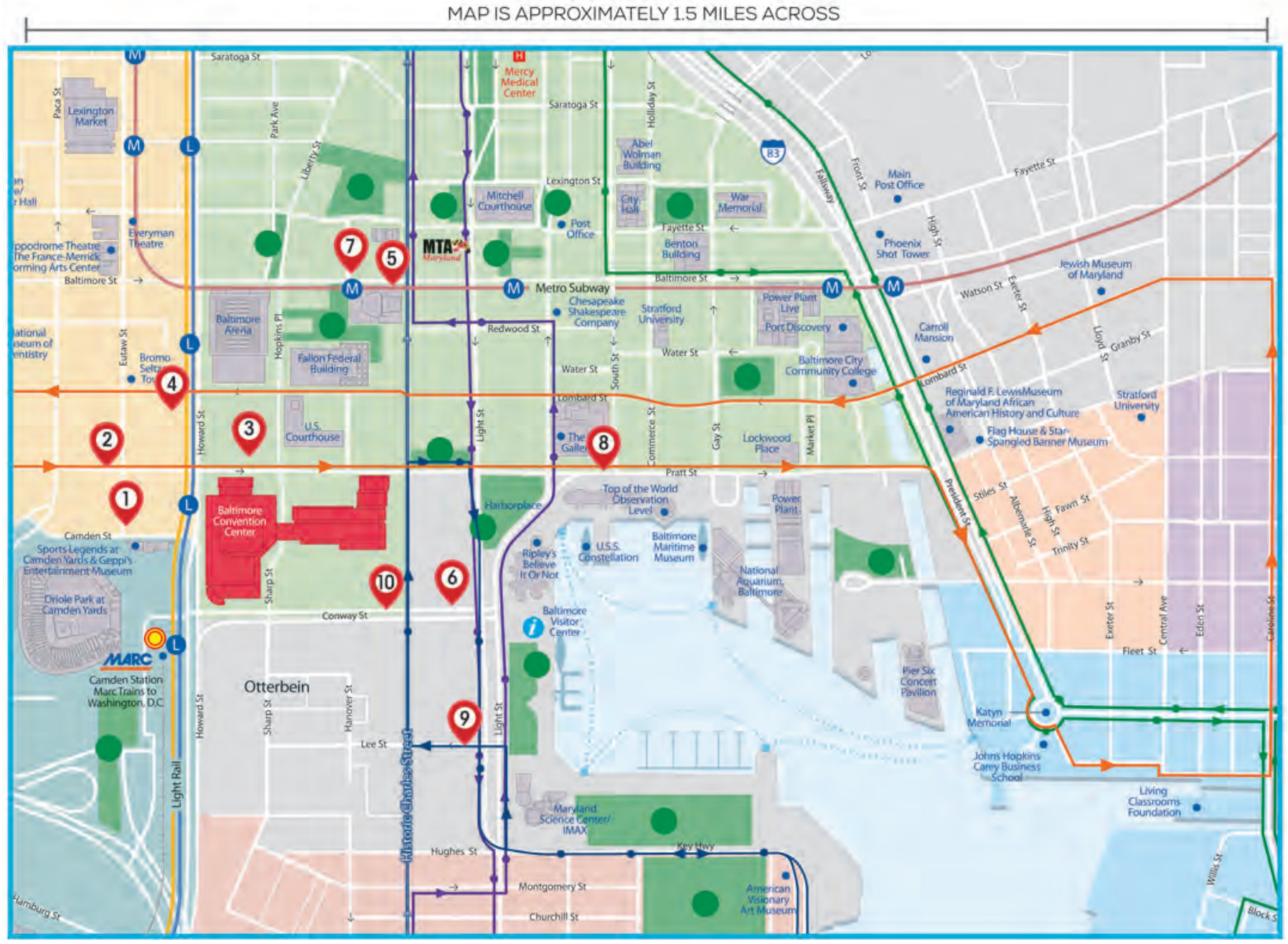




\section{Program Timetable}

This document provides an at-a-glance timetable of all scientific and social events scheduled for the JMM, so you can easily see which events may overlap and better plan your time.
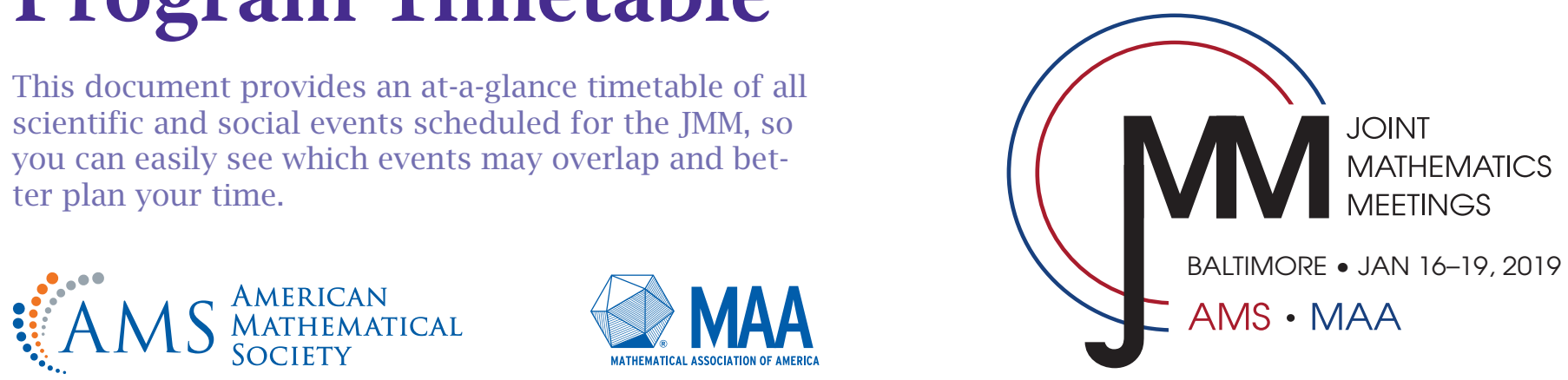

\section{Monday, January 14}

8:00 am-5:00 pm

5:00 pm-6:00 pm
AMS SHORT COURSE ON SUM OF SQUARES: THEORY AND APPLICATIONS, PART I AMS SHORT COURSE RECEPTION

\section{Tuesday, January 15}

8:00 am- 5:00 pm

8:00 am-6:30 pm

1:30 pm-10:00 pm

3:00 pm-7:00 pm

\author{
AMS SHORT COURSE ON SUM OF SQUARES: THEORY AND APPLICATIONS, PART II \\ AMS DEPARTMENT CHAIRS WORKSHOP \\ AMS COUNCIL \\ JOINT MEETINGS REGISTRATION, Pratt Street Lobby, 300 Level, BCC
}

\section{Wednesday, January 16}

7:00 am-6:00 pm

7:00 am- $5: 30 \mathrm{pm}$

7:00 am- 8:45 am

8:00 am-11:00 am 8:00 am-11:00 am

8:00 am-11:00 am 8:00 am-11:00 am 8:00 am-11:00 am 8:00 am-11:00 am 8:00 am-11:00 am 8:00 am-11:00 am 8:00 am-11:00 am 8:00 am-11:00 am 8:00 am-11:00 am 8:00 am-11:00 am 8:00 am-11:00 am 8:00 am-11:00 am 8:00 am-11:00 am 8:00 am-11:00 am
JOINT MEETINGS REGISTRATION, Pratt Street Lobby, 300 Level, BCC EMAIL CENTER

MAA MINORITY CHAIRS MEETING

\section{AMS SPECIAL SESSIONS}

Algorithmic Dimensions and Fractal Geometry, I (AMS-ASL)

How to Guard an Art Gallery and Other Discrete Mathematical Adventures (In Memory of T. S. Michael, 1960 to 2016), I

Analysis of Fractional, Stochastic, and Hybrid Dynamic Systems with Applications, I New Directions in the Theory of Complex Multiplication, I

Nonlinear Evolution Equations and Their Applications, I

Quaternions, I

Numerical Methods for PDEs and Applications, I

Recent Advancements in Mathematical Modeling of Cancer, I

Natural Resources Modeling, I

Geometry Labs United: Research, Visualization, and Outreach, I

Commutative Ring Theory: Research for Undergraduate and Early Graduate Students, I Hopf Algebras and Tensor Categories, I

Mappings on Metric and Banach Spaces with Applications to Fixed Point Theory, I

Recent Advances and Trends in Computable Structure Theory (in honor of J. Remmel), I

Financial Mathematics, I

Recent Advances in Regularity Lemmas, I 


\section{JMM 2019}

8:00 am-11:00 am 8:00 am-11:00 am 8:00 am-11:00 am

8:00 am-11:00 am

8:00 am-11:00 am

8:00 am-10:50 am 8:00 am-11:00 am

8:00 am-11:00 am 8:00 am-11:00 am 8:00 am-11:00 am 8:00 am-11:00 am 8:00 am-11:00 am 8:00 am-10:55 am

8:00 am-6:00 pm 8:00 am-10:55 am 8:00 am-10:55 am 8:00 am- 5:30 pm

9:00 am-11:00 am 9:00 am-11:00 am 9:00 am-11:00 am

9:00 am- 9:50 am

9:00 am-10:20 am 9:35 am-10:55 am

9:35 am-10:55 am

9:50 am-10:30 am

10:05 am-10:55 am

10:20 am-10:50 am

$11: 10$ am-12:00 pm

12:15 pm-5:30 pm

1:00 pm- 1:50 pm

2:15 pm-3:05 pm

2:15 pm-6:15 pm

2:15 pm-6:15 pm

2:15 pm-6:15 pm

2:15 pm-6:15 pm
Bifurcations of Difference Equations and Discrete Dynamical Systems with Applications, I A Showcase of Number Theory at Undergraduate Institutions, I

Optimal Methods in Applicable Analysis: Variational Inequalities, Low Rank Matrix Approximations, Systems Engineering, Cyber Security, I

25 years of Conferences for African-American Researchers in the Mathematical Sciences (CAARMS times 25), I

Symbolic Dynamics, I

\section{MAA INVITED PAPER SESSIONS}

Trends in Mathematical and Computational Biology

Building Successful Communities in Mathematics

\section{MAA CONTRIBUTED PAPER SESSIONS}

The Scholarship of Teaching and Learning in Collegiate Mathematics, I Inquiry-Based Learning and Teaching, I

Discrete Mathematics in the Undergraduate Curriculum-Ideas and Innovations in Teaching, I Innovative Curricular Strategies for Increasing Mathematics Majors

Mathematics and the Arts, I

\section{SIAM MINISYMPOSIUM ON ADVANCES IN MATHEMATICAL MODELING OF COMPLEX MATERIALS SYSTEMS}

PROJECT NEXT WORKSHOP

AMS CONTRIBUTED PAPER SESSIONS

MAA GENERAL CONTRIBUTED PAPER SESSIONS

EMPLOYMENT CENTER

MAA MINICOURSE \# 1: PART A Mathematical Inquiry and Writing through Sports

MAA MINICOURSE \#2: PART A Start Teaching Statistics using $R$ and RStudio

MAA MINICOURSE \#7: PART A Using Data Applications to Inspire Linear Algebra Topics in the Classroom

MAA-SIAM-AMS HRABOWSKI-GATES-TAPIA-MCBAY SESSION: LECTURE

MAA PANEL What Every Student Should Know about the JMM

MAA PANEL Mathematics Placement Trends and Innovations that Increase Equitable Access \& Success

MAA WORKSHOP NSF Funding Opportunities in the Education and Human Resources Directorate and the Division of Mathematical Sciences

MAA-SIAM-AMS HRABOWSKI-GATES-TAPIA-MCBAY PANEL Actions to increase the participation of underrepresented minority groups in mathematics.

AMS INVITED ADDRESS Algebraic, Geometric, and Topological Methods in Optimization. Jesus A. De Loera

RADICAL DASH KICKOFF MEETING

AMS-MAA INVITED ADDRESS What is the shape of a rational map? Sarah Koch

EXHIBITS AND BOOK SALES

AMS COLLOQUIUM LECTURES: LECTURE I Complex multiplication: past, present, future. Benedict H. Gross

MAA INVITED ADDRESS Symmetry, almost. Amanda Folsom

\section{AMS SPECIAL SESSIONS}

Algorithmic Dimensions and Fractal Geometry, II (AMS-ASL)

How to Guard an Art Gallery and Other Discrete Mathematical Adventures (In Memory of T. S. Michael, 1960 to 2016), II

Analysis of Fractional, Stochastic, and Hybrid Dynamic Systems with Applications, II New Directions in the Theory of Complex Multiplication, II 
2:15 pm-6:15 pm

2:15 pm-6:15 pm

2:15 pm-6:15 pm

2:15 pm-6:16 pm

2:15 pm-6:15 pm

2:15 pm-6:15 pm

2:15 pm-6:15 pm

2:15 pm-6:15 pm

2:15 pm-6:15 pm

2:15 pm-6:15 pm

2:15 pm-6:15 pm

2:15 pm-6:15 pm

2:15 pm-6:15 pm

2:15 pm-6:15 pm

2:15 pm-6:15 pm

2:15 pm-6:15 pm

2:15 pm-6:15 pm

2:15 pm-5:35 pm

2:15 pm-4:15 pm

2:15 pm-4:15 pm

2:15 pm-4:15 pm

2:15 pm-6:00 pm

2:15 pm-6:00 pm

2:15 pm-6:00 pm

2:15 pm-6:00 pm

2:15 pm-6:00 pm

2:15 pm-6:00 pm

2:15 pm-6:00 pm

2:15 pm-6:00 pm

2:15 pm-6:00 pm

2:15 pm-3:35 pm

2:15 pm-3:35 pm

2:15 pm-3:25 pm

2:15 pm-3:40 pm

2:15 pm-6:00 pm

2:15 pm-6:00 pm

2:15 pm-4:30 pm

3:20 pm-4:10 pm

3:35 pm-4:15 pm

3:45 pm-4:15 pm

4:00 pm- 5:00 pm
Nonlinear Evolution Equations and Their Applications, II

Quaternions, II

Algebraic and Geometric Methods in Discrete Optimization, I

Numerical Methods for PDEs and Applications, II

Recent Advancements in Mathematical Modeling of Cancer, II

Natural Resources Modeling, II

Geometry Labs United: Research, Visualization, and Outreach, II

Commutative Ring Theory: Research for Undergraduate and Early Graduate Students, II

Hopf Algebras and Tensor Categories, II

Mappings on Metric and Banach Spaces with Applications to Fixed Point Theory, II

Financial Mathematics, II

Recent Advances in Regularity Lemmas, II

Bifurcations of Difference Equations and Discrete Dynamical Systems with Applications, II

A Showcase of Number Theory at Undergraduate Institutions, II

Optimal Methods in Applicable Analysis: Variational Inequalities, Low Rank Matrix

Approximations, Systems Engineering, Cyber Security, II

25 years of Conferences for African-American Researchers in the Mathematical Sciences

(CAARMS times 25), II

Symbolic Dynamics, II

\section{MAA INVITED PAPER SESSIONS}

Using Research about Teaching and Learning to Inform the Preparation of Graduate Students to Teach

MAA MINICOURSE \#3: PART A Advanced Authoring in WeBWork: Turn good math problems into great ones \& submit them to the OpenProblemLibrary

MAA MINICOURSE \#4: PART A Teaching an Undergraduate Computational Science Course

MAA MINICOURSE \#9: PART A Mathematical Art from Complex Analysis

\section{MAA CONTRIBUTED PAPER SESSIONS}

The Scholarship of Teaching and Learning in Collegiate Mathematics, II

Infusing Data Science and Big Data into the Statistics Classroom

Inquiry-Based Learning and Teaching, II

Integrating Research into the Undergraduate Classroom

Discrete Mathematics in the Undergraduate Curriculum-Ideas and Innovations in Teaching, II

Mathematics and the Arts, II

Mathematics and Sports, I

Undergraduate Student TAs in Mathematics

\section{SIAM MINISYMPOSIUM ON MATHEMATICAL MODELS IN CANCER}

MAA PANEL Pursuing New Directions in Your Academic Career

MAA PANEL Mental Health in the Mathematics Profession

MAA WORKSHOP Discussing Project Ideas with NSF/EHR Program Officers, Part I

ASSOCIATION FOR WOMEN IN MATHEMATICS PANEL DISCUSSION Promoting Inclusion in STEM.

AMS CONTRIBUTED PAPER SESSIONS

MAA GENERAL CONTRIBUTED PAPER SESSIONS

AMS SPECIAL EVENT Activities in NSF's Division of Mathematical Sciences

MAA INVITED ADDRESS Title to be announced. Emmanuel Candes

MAA PANEL Impacting Mathematics Instruction Through Meaningful Collaboration with Partner Discipline Faculty

AWM BUSINESS MEETING

MAA SECTION OFFICERS 


\section{JMM 2019}

4:30 pm-6:00 pm

4:30 pm-5:50 pm

4:30 pm-5:30 pm

$5: 30 \mathrm{pm}-6: 30 \mathrm{pm}$

$5: 30 \mathrm{pm}-8: 00 \mathrm{pm}$

$6: 15 \mathrm{pm}-7: 15 \mathrm{pm}$

7:00 pm-8:30 pm

7:15 pm-8:15 pm

8:30 pm-9:20 pm

9:30 pm-11:00 pm
AMS COMMITTEE ON THE PROFESSION PANEL DISCUSSION Permanent teaching faculty in research oriented departments

TOWN HALL MEETING Spectra: Identifying Workplace Best Practices for LGBTQ Mathematicians

RECEPTION FOR UNDERGRADUATE STUDENTS

RECEPTION FOR GRADUATE STUDENTS AND FIRST-TIME PARTICIPANTS

MATHEMATICAL INSTITUTES OPEN HOUSE

SIGMAA ON THE HISTORY OF MATHEMATICS (HOM SIGMAA) BUSINESS MEETING AND RECEPTION

MATHILY, MATHILY-ER YEARLY GATHER

SIGMAA ON THE HISTORY OF MATHEMATICS (HOM SIGMAA) GUEST LECTURE

AMS JOSIAH WILLARD GIBBS LECTURE Title to be announced. Alan Perelson

ASSOCIATION FOR WOMEN IN MATHEMATICS RECEPTION AND AWARDS PRESENTATION

\section{Thursday, January 17}

7:30 am- 4:00 pm

7:30 am-5:30 pm

8:00 am-12:00 pm 8:00 am-12:00 pm 8:00 am-12:00 pm

8:00 am-12:00 pm

8:00 am-12:00 pm

8:00 am-12:00 pm

8:00 am-12:00 pm 8:00 am-12:00 pm 8:00 am-12:00 pm 8:00 am-12:00 pm 8:00 am-12:00 pm 8:00 am-12:00 pm 8:00 am-12:00 pm 8:00 am-12:00 pm 8:00 am-12:00 pm 8:00 am-12:00 pm 8:00 am-12:00 pm 8:00 am-12:00 pm 8:00 am-12:00 pm 8:00 am-12:00 pm 8:00 am-12:00 pm 8:00 am-12:00 pm

8:00 am-11:00 am 8:00 am-11:00 am
JOINT MEETINGS REGISTRATION, Pratt Street Lobby, 300 Level, BCC EMAIL CENTER

\section{AMS SPECIAL SESSIONS}

Definability and Decidability Problems in Number Theory, I (AMS-ASL)

The Mathematics of Gravity and Light (a Mathematics Research Communities Session), I

Harmonic Analysis: Recent Developments on Oscillatory Integrals (a Mathematics Research Communities Session), I

Quantum Symmetries: Subfactors and Fusion Categories (a Mathematics Research Communities Session), I

Number Theoretic Methods in Hyperbolic Geometry (a Mathematics Research Communities Session), I

Agent-based Modeling in Biological and Social Systems (a Mathematics Research Communities Session), I

New Directions in the Theory of Complex Multiplication, III

Algebraic and Geometric Methods in Discrete Optimization, II

Continued Fractions, I

Problems in Partial Differential Equations, I

Riordan Arrays, I

Stochastic Differential Equations and Applications, I

Recent Advances and Trends in Computable Structure Theory (in honor of J. Remmel), II

Analysis and Geometry of Nonlinear Evolution Equations, I

Women in Topology, I

Lattice Path Combinatorics and Applications, I

Recent Advances in Homological and Commutative Algebra, I

Research in Mathematics by Early Career Graduate Students, I

Mathematical Models in Ecology, Epidemiology, and Medicine, I

Recent Progress in Multivariable Operator Theory, I

Differential Equations on Fractals, I

The Mathematics of Historically Black Colleges and Universities (HBCUs) in the Mid-Atlantic, I

\section{MAA INVITED PAPER SESSIONS}

Inspiring Diversity in Mathematics: Culture, Community, and Collaboration

Research in Improving Undergraduate Mathematical Sciences Education: Examples Supported by the National Science Foundation's IUSE: EHR Program 
8:00 am-12:00 pm 8:00 am-12:00 pm 8:00 am-12:00 pm 8:00 am-12:00 pm 8:00 am-12:00 pm 8:00 am-12:00 pm 8:00 am-11:00 am 8:00 am-6:00 pm 8:00 am-11:55 am 8:00 am-12:00 pm 8:00 am-11:00 am 8:00 am-5:30 pm 9:00 am- 9:50 am

9:00 am-11:00 am 9:00 am-11:00 am 9:00 am-11:00 am 9:00 am-10:00 am 9:00 am-10:20 am 9:00 am-10:20 am 9:00 am-10:20 am 9:30 am- 5:30 pm 10:00 am-12:00 pm 10:00 am-12:00 pm 10:05 am-10:55 am 10:30 am-12:00 pm 10:35 am-11:55 am 11:10 am-12:00 pm $11: 10 \mathrm{am}-12: 00 \mathrm{pm}$ 1:00 pm- 1:50 pm

$1: 00 \mathrm{pm}-4: 00 \mathrm{pm}$ 1:00 pm-4:00 pm 1:00 pm-4:00 pm 1:00 pm-4:00 pm 1:00 pm-4:00 pm 1:00 pm-4:00 pm

1:00 pm-4:00 pm $1: 00 \mathrm{pm}-4: 00 \mathrm{pm}$ 1:00 pm-4:00 pm

\section{MAA CONTRIBUTED PAPER SESSIONS}

Introducing Mathematical Modeling through Competitions

Mathematics and the Life Sciences: Initiatives, Programs, Curricula

Humanistic Mathematics

Inequalities and Their Applications

Innovative and Effective Ways to Teach Linear Algebra

Research in Undergraduate Mathematics Education (RUME), I

SIAM MINISYMPOSIUM ON DATA ASSIMILATION: THEORY AND PRACTICE

PROJECT NEXT WORKSHOP

AMS CONTRIBUTED PAPER SESSIONS

MAA GENERAL CONTRIBUTED PAPER SESSIONS

PME COUNCIL MEETING

EMPLOYMENT CENTER

MAA INVITED ADDRESS The past 50 years of African Americans in the mathematical sciences. Edray Goins

MAA MINICOURSE \#2: PART B Start Teaching Statistics using $R$ and RStudio

MAA MINICOURSE \#5: PART A IBL SIGMAA Minicourse: Introduction to Inquiry-Based Learning MAA MINICOURSE \#8: PART A Dance and Mathematics

SIGMAA ON BUSINESS, INDUSTRY, AND GOVERNMENT (BIG SIGMAA) BUSINESS MEETING

MAA PANEL Connecting High School and Post High School Mathematics

MAA PANEL Preparing Math and Stats Students for Industry Careers

MAA WORKSHOP Making it Happen: Modeling in Your Differential Equations Course

EXHIBITS AND BOOK SALES

ESTIMATHON! A mindbending mixture of math and trivia.

MAA POSTER SESSION Mathematical Outreach Programs

AWM-AMS NOETHER LECTURE Dynamics of systems with low complexity. Bryna Kra SIGMAA OFFICERS MEETING

MAA WORKSHOP For Faculty on Fostering Student Engagement: Experience Classroom Practices from the MAA IP Guide

\section{MAA PROJECT NEXT LECTURE ON TEACHING AND LEARNING}

SIAM INVITED ADDRESS Recent advances in mathematical theory and scientific computation for biological fluids. Suncica Canic

AMS COLLOQUIUM LECTURES: LECTURE II Complex multiplication: past, present, future. Benedict H. Gross

\section{AMS SPECIAL SESSIONS}

Definability and Decidability Problems in Number Theory, II (AMS-ASL)

The Mathematics of Gravity and Light (a Mathematics Research Communities Session), II Harmonic Analysis: Recent Developments on Oscillatory Integrals (a Mathematics Research Communities Session), II

Quantum Symmetries: Subfactors and Fusion Categories (a Mathematics Research Communities Session), II

Number Theoretic Methods in Hyperbolic Geometry (a Mathematics Research Communities Session), II

Agent-based Modeling in Biological and Social Systems (a Mathematics Research Communities Session), II

Algebraic and Geometric Methods in Discrete Optimization, III

Continued Fractions, II

Problems in Partial Differential Equations, II 


\section{JMM 2019}

1:00 pm-4:00 pm $1: 00 \mathrm{pm}-4: 00 \mathrm{pm}$ 1:00 pm-4:00 pm 1:00 pm-4:00 pm 1:00 pm-4:00 pm 1:00 pm-4:00 pm 1:00 pm-4:00 pm 1:00 pm-4:00 pm 1:00 pm-4:00 pm 1:00 pm-4:00 pm 1:00 pm-4:00 pm 1:00 pm-4:00 pm 1:00 pm-4:00 pm

1:00 pm-4:00 pm 1:00 pm-3:00 pm 1:00 pm-3:00 pm 1:00 pm-3:00 pm

1:00 pm-4:15 pm 1:00 pm-4:15 pm 1:00 pm-4:15 pm 1:00 pm-4:15 pm 1:00 pm-4:15 pm 1:00 pm-4:15 pm

1:00 pm-4:15 pm 1:00 pm-4:10 pm 1:00 pm-2:30 pm 1:00 pm-2:20 pm 1:00 pm-2:20 pm 1:00 pm-4:00 pm 1:00 pm-2:00 pm 1:00 pm-4:00 pm 1:00 pm-3:00 pm 2:00 pm-4:00 pm

2:15 pm-3:05 pm 2:35 pm-3:55 pm 2:35 pm-3:55 pm 2:45 pm-4:15 pm

$3: 20$ pm-4:10 pm 4:25 pm-5:25 pm 5:30 pm-6:00 pm
Riordan Arrays, II Stochastic Differential Equations and Applications, II

Recent Advances and Trends in Computable Structure Theory (in honor of J. Remmel), III Analysis and Geometry of Nonlinear Evolution Equations, II

Women in Topology, II

Lattice Path Combinatorics and Applications, II

Recent Advances in Homological and Commutative Algebra, II

Recent Advances in Regularity Lemmas, III

Research in Mathematics by Early Career Graduate Students, II

Mathematical Models in Ecology, Epidemiology, and Medicine, II

Recent Progress in Multivariable Operator Theory, II

The Mathematics of Historically Black Colleges and Universities (HBCUs) in the Mid-Atlantic, II Symbolic Dynamics, III

\section{MAA INVITED PAPER SESSIONS}

Modular Forms: Aesthetics and Applications

MAA MINICOURSE \# 10: PART A Object Based Learning and the Smithsonian Learning Lab MAA MINICOURSE \# 11: PART A Object Based Learning and the Smithsonian Learning Lab MAA MINICOURSE \#9: PART B Mathematical Art from Complex Analysis

\section{MAA CONTRIBUTED PAPER SESSIONS}

Integrated STEM Instruction in Undergraduate Mathematics

Revitalizing Complex Analysis

The EDGE (Enhancing Diversity in Graduate Education) program: Pure and Applied talks by Women Math Warriors

Formative and Summative Assessment of Mathematical Communication and Conceptual Understanding

Mathematics and Sports, II

Touch it, Feel it, Learn it: Tactile Learning Activities in the Undergraduate Mathematics Classroom

Research in Undergraduate Mathematics Education (RUME), II

\section{SIAM MINISYMPOSIUM ON HUMAN FACTORS IN MATHEMATICS EDUCATION}

\section{AMS COMMITTEE ON EDUCATION PANEL DISCUSSION}

MAA PANEL Advising and Mentorship: Fostering Successful Students

MAA PANEL Coping Professionally with Unprofessional Behavior

AMS CONTRIBUTED PAPER SESSIONS

THE DOLCIANI AWARD LECTURES

MAA GENERAL CONTRIBUTED PAPER SESSIONS

SUMMER PROGRAM FOR WOMEN IN MATHEMATICS (SPWM) REUNION

MAA POSTER SESSION: PROJECTS SUPPORTED BY THE NSF DIVISION OF UNDERGRADUATE EDUCATION

AMS INVITED ADDRESS Title to be announced. Peter Oszvath

MAA PANEL Pathways to Leadership

MAA WORKSHOP How to Talk About Math So People Want to Listen

AMS EDUCATION AND DIVERSITY DEPARTMENT PANEL Bridge-to-PhD and Postbac Programs Working to Open Doors for Students from Underrepresented Groups

AMS INVITED ADDRESS Title to be announced. Lior Pachter JOINT PRIZE SESSION

SIGMAA ON THE PHILOSOPHY OF MATHEMATICS (POM SIGMAA) RECEPTION 
5:30 pm-6:30 pm

5:30 pm-7:30 pm

5:30 pm-6:30 pm

5:30 pm- 7:00 pm

5:30 pm- 7:00 pm

5:30 pm- 7:30 pm

6:00 pm-6:15 pm

6:00 pm- 7:30 pm

6:00 pm-8:00 pm

6:00 pm-8:00 pm

6:00 pm-8:00 pm

6:15 pm- 7:05 pm

6:30 pm-8:00 pm

6:30 pm-8:30 pm

8:15 pm-9:45 pm
JOINT PRIZE SESSION RECEPTION

ASSOCIATION OF CHRISTIANS IN THE MATHEMATICAL SCIENCES RECEPTION AND LECTURE

BUDAPEST SEMESTERS IN MATHEMATICS ANNUAL ALUMNI REUNION

MAA TWO-YEAR COLLEGE RECEPTION

UNIVERSITY OF MICHIGAN MATHEMATICS ALUMNI AND FRIENDS RECEPTION

UNIVERSITY OF TENNESSEE MATHEMATICS DEPARTMENT ALUMNI AND FRIENDS RECEPTION

SIGMAA ON THE PHILOSOPHY OF MATHEMATICS (POM SIGMAA) BUSINESS MEETING

SIGMAA ON QUANTITATIVE LITERACY (SIGMAA QL) JOINT GUEST LECTURE AND RECEPTION

SPECTRA RECEPTION FOR LGBT MATHEMATICIANS AND ALLIES

NSA'S WOMEN IN MATHEMATICS SOCIETY NETWORKING SESSION

UNIVERSITY OF WATERLOO FACULTY OF MATHEMATICIANS ALUMNI \& FRIENDS RECEPTION

SIGMAA ON THE PHILOSOPHY OF MATHEMATICS (POM SIGMAA) GUEST LECTURE MSRI RECEPTION FOR CURRENT AND FUTURE DONORS

PROMYS AND ROSS RECEPTION FOR ALUMNI AND FRIENDS

KNITTING CIRCLE Knitting Circle: Bring a project (knitting/crochet/tatting/beading/etc.) and chat with other mathematical crafters

\section{Friday, January 18}

7:17 am- 9:00 am

7:30 am-4:00 pm

7:30 am- 5:30 pm

8:00 am-11:00 am

8:00 am-11:00 am 8:00 am-11:00 am 8:00 am-11:00 am 8:00 am-11:00 am 8:00 am-11:00 am 8:00 am-11:00 am 8:00 am-11:00 am 8:00 am-11:00 am 8:00 am-11:00 am 8:00 am-11:00 am 8:00 am-11:00 am 8:00 am-11:00 am 8:00 am-11:00 am 8:00 am-11:00 am

8:00 am-11:00 am 8:00 am-11:00 am 8:00 am-11:00 am 8:00 am-11:00 am
YP1 7 HCSSIM REUNION BREAKFAST

JOINT MEETINGS REGISTRATION, Pratt Street Lobby, 300 Level, BCC EMAIL CENTER

\section{AMS SPECIAL SESSIONS}

Research in Mathematics by Undergraduates and Students in Post-Baccalaureate Programs, I (AMS-MAA-SIAM)

Stochastic Analysis and Applications in Finance, Actuarial Science and Related Fields, I

Mathematics in the Realm of Cyber Research, I

Recent Advances in Inverse Problems and Imaging, I

Advances in Operator Theory, Operator Algebras, and Operator Semigroups, I

Mathematical Analysis in Fluid Dynamics, I

Analytic Number Theory, I

Geometric and Topological Combinatorics, I

Harmonic Analysis, Partial Differential Equations, and Applications, I

Multiscale Problems in the Calculus of Variations, I

Geometry and Dynamics of Continued Fractions, I

Topology, Structure and Symmetry in Graph Theory, I

Algebraic Structures Motivated by Knot Theory, I

Arithmetic Statistics, I

If You Build It They Will Come: Presentations by Scholars in the National Alliance for Doctoral Studies in the Mathematical Sciences, I

Low Complexity Models in Data Analysis and Machine Learning, I

Recent Advances in Biological Modeling and Related Dynamical Analysis, I

Counting Methods in Number Theory, I

Geometric and Topological Generalization of Groups, I 


\section{JMM 2019}

8:00 am-11:00 am 8:00 am-11:00 am

8:00 am-11:00 am

8:00 am-11:00 am

8:00 am-11:00 am 8:00 am-11:00 am 8:00 am-11:00 am 8:00 am-1 1:00 am 8:00 am-11:00 am 8:00 am-11:00 am 8:00 am-10:55 am

8:00 am- 9:20 am 8:00 am-6:00 pm 8:00 am-10:55 am 8:00 am-11:00 am 8:00 am- 5:30 pm 8:30 am-10:30 am

9:00 am- 9:50 am

9:00 am- 9:50 am

9:00 am-11:00 am 9:00 am-11:00 am

9:00 am-11:00 am

9:00 am-11:00 am

9:30 am-5:30 pm

9:35 am-10:55 am

9:45 am-10:55 am 10:00 am-10:50 am

10:05 am-10:55 am

10:30 am-11:00 am

11:10 am-12:00 pm

12:00 pm-1:00 pm

1:00 pm-1:50 pm
Mathematical Investigations of Spatial Ecology and Epidemiology, I

Differential Equations on Fractals, II

\section{MAA INVITED PAPER SESSIONS}

The Past 50 Years of African Americans in the Mathematical Sciences

\section{MAA CONTRIBUTED PAPER SESSIONS}

Open Educational Resources: Combining Technological Tools and Innovative Practices to Improve Student Learning

Mathematical Experiences and Projects in Business, Industry, and Government (BIG)

Ethnomathematics: Ideas and Innovations in the Classroom

Philosophy of Mathematics

Inquiry-Based Learning and Teaching, III

Technology and Resources in Statistics Education, I

Research in Undergraduate Mathematics Education (RUME), III

SIAM MINISYMPOSIUM ON RECENT ADVANCES IN MATHEMATICAL THEORY AND SCIENTIFIC COMPUTATION FOR BIOLOGICAL FLUIDS.

MAA PANEL Advanced Placement Calculus and Student Understanding

PROJECT NEXT WORKSHOP

AMS CONTRIBUTED PAPER SESSIONS

MAA GENERAL CONTRIBUTED PAPER SESSIONS

EMPLOYMENT CENTER

AMS-MAA GRAD SCHOOL FAIR Undergrads! Take this opportunity to meet representatives from mathematical science graduate programs.

MAA INVITED ADDRESS A mathematical journey of culture, community, and collaboration. Pamela Harris

ASL INVITED ADDRESS Colorings of finite subgraphs of the Henson graphs. Natasha Dobrinen

MAA MINICOURSE \# 1: PART B Mathematical Inquiry and Writing through Sports

MAA MINICOURSE \#6: PART A Visualizing Multivariable Calculus \& Differential Equations using CalcPlot3D

MAA MINICOURSE \#7: PART B Using Data Applications to Inspire Linear Algebra Topics in the Classroom

MAA POSTER SESSION Recreational Mathematics: Puzzles, Card Tricks. Games, and Gambling EXHIBITS AND BOOK SALES

MAA PANEL Increasing Diversity and Retention in STEM Through Math-Focused First-Year Seminars

MAA WORKSHOP Discussing Project Ideas with NSF/EHR Program Officers, Part II

ASL INVITED ADDRESS 35 years later: A fresh perspective on classifiable theories. Michael C Laskowski

AMS INVITED ADDRESS The Roaring Twenties in American Mathematics. Karen Hunger Parshall

RADICAL DASH PRIZE SESSION

AMS-MAA INVITED ADDRESS Miracles of Algebraic Graph Theory Daniel Spielman BUDAPEST SEMESTERS IN MATHEMATICS EDUCATION (BSME) INFORMATIONAL SESSION AMS COLLOQUIUM LECTURES: LECTURE III Complex multiplication: past, present, future. Benedict H. Gross 
1:00 pm-1:50 pm

$1: 00$ pm-4:45 pm

1:00 pm-6:00 pm

1:00 pm-6:00 pm

1:00 pm-6:00 pm

1:00 pm-6:00 pm

1:00 pm-6:00 pm

1:00 pm-6:00 pm

1:00 pm-6:00 pm

1:00 pm-6:00 pm

1:00 pm-6:00 pm

1:00 pm-6:00 pm

1:00 pm-6:00 pm

$1: 00 \mathrm{pm}-6: 00 \mathrm{pm}$

1:00 pm-6:00 pm

1:00 pm-6:00 pm

1:00 pm-6:00 pm

1:00 pm-6:00 pm

$1: 00$ pm-6:00 pm

1:00 pm-6:00 pm

1:00 pm-6:00 pm

1:00 pm-6:00 pm

$1: 00$ pm-3:30 pm

1:00 pm-6:00 pm

1:00 pm-3:00 pm

1:00 pm-3:00 pm

$1: 00$ pm-3:00 pm

1:00 pm- 5:00 pm

1:00 pm-5:00 pm

$1: 00 \mathrm{pm}-5: 00 \mathrm{pm}$

1:00 pm-5:00 pm

$1: 00 \mathrm{pm}-6: 00 \mathrm{pm}$

$1: 00 \mathrm{pm}-6: 00 \mathrm{pm}$

1:00 pm-6:00 pm

1:00 pm- 5:00 pm

1:00 pm-2:20 pm

1:00 pm-6:00 pm

1:00 pm-6:00 pm
MAA LECTURE FOR STUDENTS Drawing conclusions from drawing a square. Annalisa Crannell

\section{CURRENT EVENTS BULLETIN}

\section{AMS SPECIAL SESSIONS}

Research in Mathematics by Undergraduates and Students in Post-Baccalaureate Programs, II (AMS-MAA-SIAM)

Stochastic Analysis and Applications in Finance, Actuarial Science and Related Fields, II

Mathematics in the Realm of Cyber Research, II

Recent Advances in Inverse Problems and Imaging, II

Orthogonal Polynomials, Quantum Probability, Harmonic and Stochastic Analysis, I

Advances in Operator Theory, Operator Algebras, and Operator Semigroups, II

Mathematical Analysis in Fluid Dynamics, II

Analytic Number Theory, II

Geometric and Topological Combinatorics, II

Harmonic Analysis, Partial Differential Equations, and Applications, II

Multiscale Problems in the Calculus of Variations, II

Geometry and Dynamics of Continued Fractions, II

Topology, Structure and Symmetry in Graph Theory, II

Algebraic Structures Motivated by Knot Theory, II

Arithmetic Statistics, II

If You Build It They Will Come: Presentations by Scholars in the National Alliance for Doctoral Studies in the Mathematical Sciences, II

Low Complexity Models in Data Analysis and Machine Learning, II

Recent Advances in Biological Modeling and Related Dynamical Analysis, II

Geometric and Topological Generalization of Groups, II

Mathematical Investigations of Spatial Ecology and Epidemiology, II

\section{MAA INVITED PAPER SESSIONS}

Mathematical Thinking for Modern Data Science Problems

\section{AMS SPECIAL SESSIONS}

History of Mathematics, I (AMS-MAA-ICHM)

MAA MINICOURSE \# 12: PART A Keep Teaching Statistics using $R$ and RStudio

MAA MINICOURSE \#3: PART B Advanced Authoring in WeBWorK: Turn good math problems into great ones \& submit them to the OpenProblemLibrary

MAA MINICOURSE \#4: PART B Teaching an Undergraduate Computational Science Course

\section{MAA CONTRIBUTED PAPER SESSIONS}

Mathematical Themes in a First-Year Seminar

Good Math from Bad: Crackpots, Cranks, and Progress

It's Circular: Conjecture, Compute, Iterate

The Teaching and Learning of Undergraduate Ordinary Differential Equations

Inquiry-Based Learning and Teaching, IV

Research in Undergraduate Mathematics Education (RUME), IV

SIAM MINISYMPOSIUM ON RECENT DEVELOPMENTS IN NUMERICAL METHODS FOR FLUIDS.

NAM HAYNES-GRANVILLE-BROWNE SESSION OF PRESENTATIONS BY RECENT DOCTORAL RECIPIENTS

MAA PANEL MAA Instructional Practices Guide's Value for Your Department

AMS CONTRIBUTED PAPER SESSIONS

MAA GENERAL CONTRIBUTED PAPER SESSIONS 


\section{JMM 2019}

1:30 pm-3:30 pm

2:00 pm-2:50 pm

2:15 pm-4:00 pm

2:30 pm-3:45 pm

2:30 pm-4:00 pm

3:00 pm-3:50 pm

3:30 pm-6:00 pm

4:00 pm- 5:50 pm

4:30 pm- 5:15 pm

4:30 pm-6:00 pm

4:30 pm-6:30 pm

5:00 pm-6:15 pm

5:00 pm- 7:00 pm

5:15 pm-6:00 pm

5:30 pm- 7:30 pm

6:00 pm- 7:00 pm

6:00 pm- 7:30 pm

6:00 pm- 7:00 pm

6:00 pm- 7:00 pm

6:00 pm-8:40 pm

6:00 pm-8:00 pm

6:00 pm-7:30 pm

6:30 pm- 7:30 pm

6:30 pm-7:30 pm

6:30 pm-7:30 pm

7:00 pm-8:00 pm

7:00 pm-8:30 pm

7:00 pm-8:30 pm

7:30 pm-8:30 pm

7:45 pm-8:35 pm

8:00 pm-10:00 pm

8:00 pm-10:00 pm
MAA POSTER SESSION Activities for Teaching Multivariable Thinking through Data Visualization in introductory Statistics

ASL INVITED ADDRESS Some questions and results for classical algebraic structures. Sergey Goncharov

ROCKY MOUNTAIN MATHEMATICS CONSORTIUM BOARD OF DIRECTORS MEETING PRESENTATIONS BY MAA TEACHING AWARD RECIPIENTS

AMS COMMITTEE ON SCIENCE POLICY PANEL DISCUSSION

ASL INVITED ADDRESS Valuations and o-minimality. Jana Marikova

MAA CONTRIBUTED PAPER SESSIONS

Technology and Resources in Statistics Education, II

ASL CONTRIBUTED PAPER SESSION

SIGMAA ON BUSINESS, INDUSTRY, AND GOVERNMENT (BIG SIGMAA) GUEST LECTURE MAA STUDENT POSTER SESSION

AMS CONGRESSIONAL FELLOWSHIP SESSION

AWM WORKSHOP: POSTER PRESENTATIONS BY WOMEN GRADUATE STUDENTS AND RECEPTION

MAA PANEL Advising Actuarial Science Students

SIGMAA ON BUSINESS, INDUSTRY, AND GOVERNMENT (BIG SIGMAA) RECEPTION

TEXAS A \& M UNIVERSITY MATHEMATICS DEPARTMENT ALUMNI, STUDENT, AND FACULTY RECEPTION

SIGMAA ON MATHEMATICAL AND COMPUTATIONAL BIOLOGY(BIO SIGMAA) RECEPTION AND BUSINESS MEETING

SIGMAA ON MATHEMATICS INSTRUCTION USING THE WEB (WEB SIGMAA) BUSINESS MEETING, RECEPTION, AND GUEST LECTURE

MATHEMATICALLY BENT THEATER Performed by Colin Adams and the Mobiusbandaid Players.

AMS MATHEMATICAL REVIEWS RECEPTION

NAM RECEPTION AND BANQUET

UNIVERSITY OF CALIFORNIA, SAN DIEGO RECEPTION FOR MATHEMATICS

UNIVERSITY OF ILLINOIS AT URBANA-CHAMPAIGN DEPARTMENT OF MATHEMATICS ALUMNI RECEPTION

SIGMAA ON INQUIRY BASED LEARNING BUSINESS MEETING

SIGMAA ON MATH CIRCLES FOR STUDENTS AND TEACHERS BUSINESS MEETING

SIGMAA ON STATISTICS EDUCATION BUSINESS MEETING

SIGMAA ON MATHEMATICAL AND COMPUTATIONAL BIOLOGY GUEST LECTURE

MAA SPECIAL PRESENTATION: Poetry Reading

SCUDEM GATHERING AND REUNION

SIGMAA ON STATISTICS EDUCATION GUEST LECTURE

NAM COX-TALBOT ADDRESS A Seat at the Table: Equity and Social Justice in Mathematics Education. Talitha Williams

PROJECT NEXT RECEPTION All Project NEXT Fellows, consultants, and other friends of Project NEXT are invited.

BACKGAMMON! Learn to play backgammon from expert players. 


\section{Saturday, January 19}

7:30 am-1:00 pm

7:30 am- 1:00 pm

8:00 am-10:00 am

8:00 am-12:00 pm

8:00 am-12:00 pm

8:00 am-12:00 pm

8:00 am-12:00 pm

8:00 am-12:00 pm

8:00 am-12:00 pm

8:00 am-12:00 pm

8:00 am-12:00 pm

8:00 am-12:00 pm

8:00 am-12:00 pm

8:00 am-12:00 pm

8:00 am-12:00 pm

8:00 am-12:00 pm

8:00 am-12:00 pm

8:00 am-12:00 pm

8:00 am-12:00 pm

8:00 am-12:00 pm

8:00 am-12:00 pm

8:00 am-12:00 pm

8:00 am-11:00 am

8:00 am-12:00 pm 8:00 am-10:00 am

8:00 am-12:00 pm 8:00 am-12:00 pm 8:00 am-12:00 pm 8:00 am-12:00 pm 8:00 am-12:00 pm

8:00 am-12:00 pm

8:00 am- 5:00 pm 8:00 am-6:00 pm $8: 00 \mathrm{am}-12: 00 \mathrm{pm}$ 8:00 am-12:00 pm
JOINT MEETINGS REGISTRATION, Pratt Street Lobby, 300 Level, BCC EMAIL CENTER

\section{AMS SPECIAL SESSIONS}

Research in Mathematics by Undergraduates and Students in Post-Baccalaureate Programs, III (AMS-MAA-SIAM)

Advances in Quantum Walks, Quantum Simulations, and Related Quantum Theory, I

Mathematicians at Sea (in the Sky, or on Land): Defense Applications of Mathematics, I

Enumerative Combinatorics, I

Orthogonal Polynomials, Quantum Probability, Harmonic and Stochastic Analysis, II

Using Modeling to Motivate the Study of Differential Equations, I

Latinx in Math, I

Group Representation Theory and Character Theory, I

Advances and Applications in Integral and Differential Equations, I

Algebraic, Discrete, Topological and Stochastic Approaches to Modeling in Mathematical Biology, I

Statistical, Variational, and Learning Techniques in Image Analysis and their Applications to Biomedical, Hyperspectral, and Other Imaging, I

Network Science, I

Geometry of Representation Spaces, I

Number Theory, Arithmetic Geometry, and Computation, I

Topological Data Analysis: Theory and Applications, I

Advances by Early Career Women in Discrete Mathematics, I

Not KNerds: A Community for Knot Theory, I

Mathematics of Coding Theory and Applications, I

Partition Theory and Related Topics, I

Localization and Delocalization for Disordered Quantum Systems, I

MAA INVITED PAPER SESSIONS

Beauty and Art from Research Mathematics

AMS SPECIAL SESSIONS

History of Mathematics, II (AMS-MAA-ICHM)

MAA MINICOURSE \# 11: PART B Object Based Learning and the Smithsonian Learning Lab

\section{MAA CONTRIBUTED PAPER SESSIONS}

Approaches to Mathematics Remediation in Baccalaureate-Granting Institutions

Fostering Creativity in Undergraduate Mathematics Courses

Incorporating Programming and Computing in the Math Major Curriculum

Innovative Pathways to Quantitative Literacy

Inclusive Excellence-Attracting, Involving, and Retaining Women and Underrepresented Groups in Mathematics

SIAM MINISYMPOSIUM ON ANALYTICAL TECHNIQUES IN IMAGING ELECTRICAL PROPERTIES OF TISSUE IN COUPLED PHYSICS MODELS.

AWM WORKSHOP:WINCOMPTOP: APPLIED AND COMPUTATIONAL TOPOLOGY

PROJECT NEXT WORKSHOP

AMS CONTRIBUTED PAPER SESSIONS

MAA GENERAL CONTRIBUTED PAPER SESSIONS 


\section{JMM 2019}

8:30 am-10:50 am Research in Undergraduate Mathematics Education: Highlights from the Annual SIGMAA on RUME Conference

9:00 am- 9:50 am

AMS INVITED ADDRESS Title to be announced. Lillian Pierce

9:00 am- 9:50 am

9:00 am-11:00 am

9:00 am-11:00 am

9:00 am-10:20 am

9:00 am- 9:50 am

9:00 am-12:00 pm

9:45 am-10:55 am

10:00 am-10:50 am

10:00 am-10:50 am

10:00 am-12:00 pm

10:00 am-10:50 am

ASL INVITED ADDRESS Computable aspects of homogeneous structures. Douglas Cenzer

MAA MINICOURSE \#5: PART B IBL SIGMAA Minicourse: Introduction to Inquiry-Based Learning

MAA MINICOURSE \#8: PART B Dance and Mathematics

MAA PANEL Calculus before the Senior Year of High School: Issues and Options

NAM PANEL DISCUSSION NAM 2019-2069: Where Do We Go from Here?

EXHIBITS AND BOOK SALES

MAA WORKSHOP Calculus: Near-Numbers

MAA INTERACTIVE LECTURE FOR STUDENTS AND TEACHERS

ASL INVITED ADDRESS A forcing axiom for a non-special Aronszajn tree. John Krueger

\section{AMS SPECIAL SESSIONS}

Counting Methods in Number Theory, II

NAM BUSINESS MEETING

10:00 am-10:50 am

10:30 am-11:50 am

11:00 am-12:00 pm

11:10 am-11:40 am

$11: 45 \mathrm{am}-12: 15 \mathrm{pm}$

1:00 pm- 1:50 pm

1:00 pm- 1:50 pm

1:00 pm-6:00 pm

1:00 pm-6:00 pm

1:00 pm-6:00 pm

1:00 pm-6:00 pm

1:00 pm-6:00 pm

1:00 pm-6:00 pm

1:00 pm-6:00 pm

1:00 pm-6:00 pm

1:00 pm-6:00 pm

1:00 pm-6:00 pm

1:00 pm-6:00 pm

1:00 pm-6:00 pm

1:00 pm-6:00 pm

1:00 pm-6:00 pm

$1: 00 \mathrm{pm}-6: 00 \mathrm{pm}$

1:00 pm-6:00 pm

1:00 pm-6:00 pm

1:00 pm-6:00 pm

MAA INVITED ADDRESS The Inclusion Principle: the importance of community in mathematics. Deanna Haunsperger

MAA PANEL Listening and Responding to Students' Thinking, from Elementary to Undergraduate Mathematics

\section{MATHEMATI-CON PRESENTS: SHOWTIME!}

\section{MAA BUSINESS MEETING}

\section{AMS BUSINESS MEETING}

NAM CLAYTOR-WOODARD LECTURE On Mathematical Problems in Geometric Optics Henok Mawi

ASL INVITED ADDRESS Fifty years in the model theory of differential fields. David Marker

\section{AMS SPECIAL SESSIONS}

Research in Mathematics by Undergraduates and Students in Post-Baccalaureate Programs, IV (AMS-MAA-SIAM)

Advances in Quantum Walks, Quantum Simulations, and Related Quantum Theory, II

Mathematicians at Sea (in the Sky, or on Land): Defense Applications of Mathematics, II

Enumerative Combinatorics, II

Using Modeling to Motivate the Study of Differential Equations, II

Latinx in Math, II

Group Representation Theory and Character Theory, II

Advances and Applications in Integral and Differential Equations, II

Algebraic, Discrete, Topological and Stochastic Approaches to Modeling in Mathematical Biology,

Statistical, Variational, and Learning Techniques in Image Analysis and their Applications to Biomedical, Hyperspectral, and Other Imaging, II

Network Science, II

Geometry of Representation Spaces, II

Number Theory, Arithmetic Geometry, and Computation, II

Counting Methods in Number Theory, III

Topological Data Analysis: Theory and Applications, II

Advances by Early Career Women in Discrete Mathematics, II

Not KNerds: A Community for Knot Theory, II

Mathematics of Coding Theory and Applications, II 
1:00 pm-6:00 pm

1:00 pm-6:00 pm

$1: 00 \mathrm{pm}-4: 40 \mathrm{pm}$

1:00 pm-6:00 pm

1:00 pm-3:00 pm

$1: 00 \mathrm{pm}-3: 00 \mathrm{pm}$

1:00 pm-3:00 pm

1:00 pm-5:00 pm

$1: 00 \mathrm{pm}-5: 30 \mathrm{pm}$

$1: 00 \mathrm{pm}-2: 45 \mathrm{pm}$

1:00 pm-6:00 pm

1:00 pm-6:00 pm

3:00 pm-4:00 pm

7:00 pm-9:30 pm
Partition Theory and Related Topics, II

Localization and Delocalization for Disordered Quantum Systems, II

\section{MAA INVITED PAPER SESSIONS}

Mathematics and Policy

\section{AMS SPECIAL SESSIONS}

History of Mathematics, III (AMS-MAA-ICHM)

MAA MINICOURSE \# 10: PART B Object Based Learning and the Smithsonian Learning Lab

MAA MINICOURSE \# 12: PART B Keep Teaching Statistics using $R$ and RStudio

MAA MINICOURSE \#6: PART B Visualizing Multivariable Calculus \& Differential Equations using CalcPlot3D

\section{MAA CONTRIBUTED PAPER SESSIONS}

Meaningful Modeling in the First Two Years of College

SIAM MINISYMPOSIUM ON FLOW-INDUCED (IN)STABILITY OF ELASTIC STRUCTURES

AMS SPECIAL PRESENTATION Who Wants to Be a Mathematician-Championship Contest.

AMS CONTRIBUTED PAPER SESSIONS

MAA GENERAL CONTRIBUTED PAPER SESSIONS

MAA-AMS-SIAM GERALD AND JUDITH PORTER PUBLIC LECTURE Big data, inequality, and democracy. Cathy O'Neil

2019 AMS “UNTIL NEXT TIME” SOCIAL, Maryland Science Center

The times noted above and in the full JMM Program listing were current as of press time.

For the most up to date scheduling information, please see:

http://jointmathematicsmeetings.org/meetings/national/jmm2019/2217_timetab1e.htm1 


\section{AMS Mathematical Moments 8. Mathematics of Planet Earth}
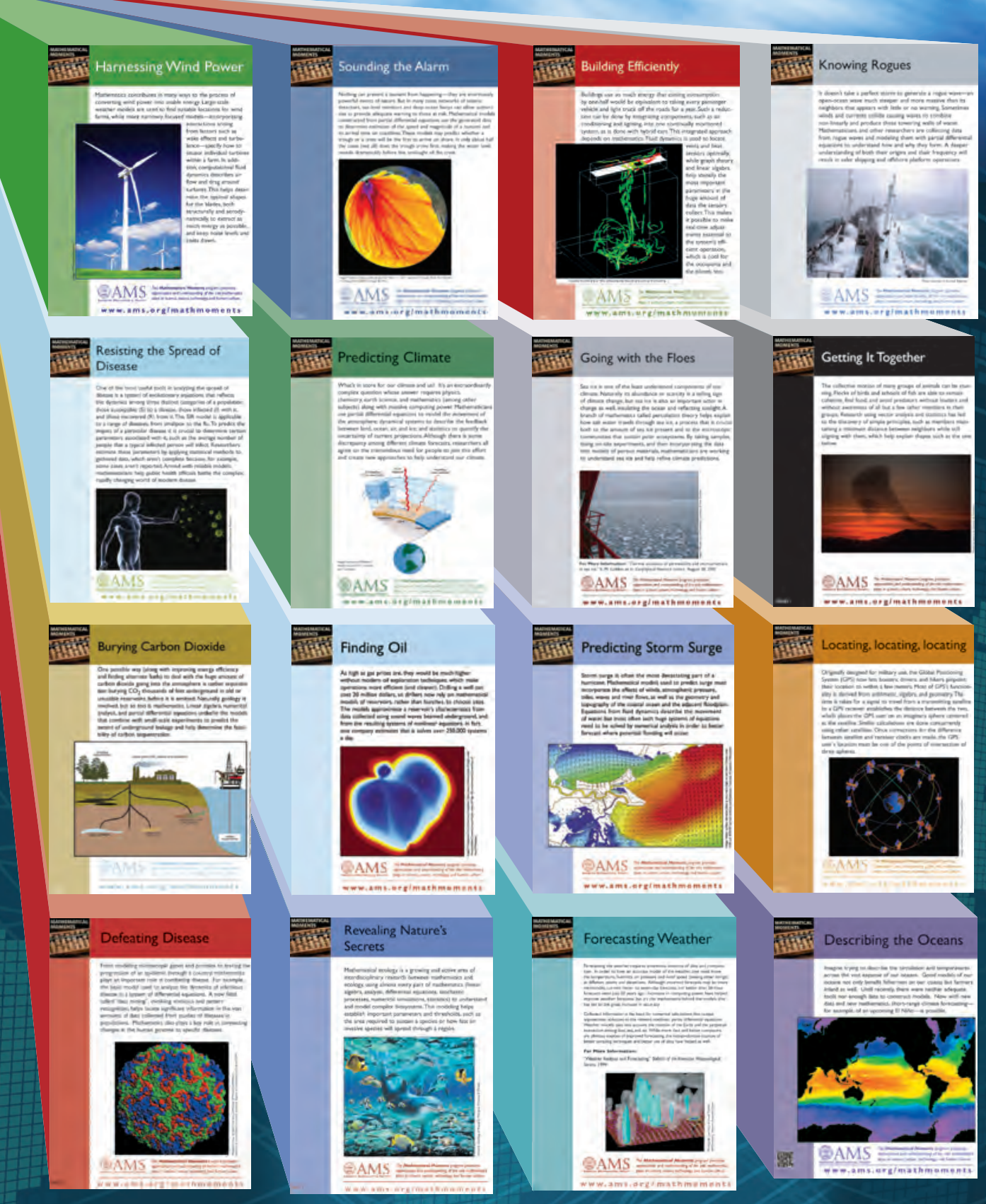

$\stackrel{4}{0}$ A

See how math is connected to understanding our planet and its many dynamic processes at www.ams.org/mathmoments and ams.org/samplings/mpe-2013. 


\section{Joint Mathematics Meetings Advance Registration/Housing Form}

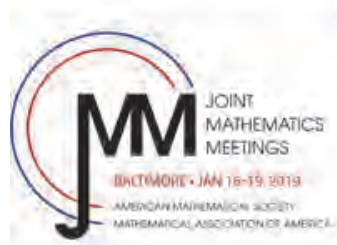

Name

(please print your name as you would like it to appear on your badge)

Mailing Address

Telephone Fax:

In case you have an emergency at the meeting: Day \#.___ Evening \#

Email Address

Additional email address for receipt

Acknowledgment of this registration and any hotel reservations will be sent to the email address(es) given here Check this box to receive a copy in U.S. Mail: $\square$

Affiliation for badge (company/university) Nonmathematician guest badge name: (Note lee ot USS 22)

PLEASE NOTE THAT BADGES WILL NOT BE MAILED IN ADVANCE FOR THIS MEETING. YOU MAY OPT TO HAVE YOUR PROGRAM MAILED ON DEC. 12 (SEE BELOW)

\section{Registration Fees}

Membership please $\checkmark$ all that apply. First row is eligible to register as a member For undergraduate students, membership in PME and KME also applies. Undergraduate Students Only: $\square$ PME $\square \mathrm{KME}$ Other Societies: $\square$ AWM $\square$ NAM $\square$ YMN $\square$ AMATYC

\begin{tabular}{|c|c|c|c|c|c|c|}
\hline \multicolumn{2}{|r|}{ Joint Meetings } & \multicolumn{2}{|c|}{ Dec 27} & \multicolumn{2}{|c|}{ at mtg } & \multirow[t]{2}{*}{ Subtotal } \\
\hline$\square$ & Member AMS, MAA, ASL, CMS, or SIAM & USS & 345 & US\$ 45 & 455 & \\
\hline$\square$ & Nonmember & USS & 548 & US\$ 6 & 599 & \\
\hline$\square$ & Graduate Student Member (AMS, MAA & & & & & \\
\hline & ASL, CMS, or SIAM) & US\$ & 78 & US\$ & 90 & \\
\hline$\square$ & Graduate Student (Nonmember) & uss & 124 & US\$ 13 & 137 & \\
\hline 口 & Undergraduate Student (Member AMS. & & & & & \\
\hline & ASL, CMS, MAA, PME, KME, or SIAM) & US\$ & 78 & US\$ & 90 & \\
\hline$\square$ & Undergraduate Student (Nonmember) & USS & 124 & US\$ 13 & 137 & \\
\hline$\vec{\square}$ & High School Student & USS & 7 & US\$ & 15 & \\
\hline$\square$ & Unemployed & USS & 78 & US\$ & 90 & \\
\hline$\square$ & Temporarily Employed & USS & 281 & US\$ 3 & 322 & \\
\hline $\bar{\square}$ & Developing Countries Special Rate & USS & 78 & USS & 90 & \\
\hline$\square$ & Emeritus Member of AMS or MAA & USS & 78 & US\$ & 90 & \\
\hline$\square$ & High School Teacher & US\$ & 78 & US\$ & 90 & \\
\hline$\vec{\square}$ & Librarian & US\$ & 78 & US\$ & 90 & \\
\hline$\square$ & Press & USS & 0 & US\$ & 0 & \\
\hline$\square$ & Exhibitor (Commercial) & USS & 0 & US\$ & 0 & \\
\hline$\square$ & Artist Exhibitor (work in JMM Art Exhibit) & USS & 0 & USS & 0 & \\
\hline 口 & Nonmathematician Guest & & & & & \\
\hline & of registered mathematician & USS & 22 & USS & 22 & \\
\hline
\end{tabular}

AMS Short Course: Sum of Squares (1/16-1/19)

Member of AMS USS 124 US\$ 158

Nonmember US\$ 190 US\$225

$\square$ Student, Unemployed, Emeritus US\$ 72 US\$ 93

MAA Minicourses (see listing in text)

I would like to attend: $\square$ One Minicourse $\square$ Two Minicourses

Please enroll me in MAA Minicourse(s) \#

Price: US\$ 100 for each minicourse.

(For more than 2 minicourses, call or email the MMSB.) and \#

ㅁ Graduate Program Table (includes table, posterboard \& electricity)

Dept. or Program to be represented (write below or email)

Receptions \& Banquets

\begin{tabular}{l}
58 \\
93 \\
\hline
\end{tabular}

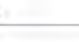
$\square$ AMS \& MAA $\square$ AMS but not MAA $\square$ MAA but notAMS $\square$ ASL $\square$ CMS $\square$ SIAM

Graduate School Fair Table

\section{Payment}

Registration \& Event Total (total from column on left)

Hotel Deposit (only if paying by check)

II you send a hotel deposit check, the

deadline for this form is December 1 .

Total Amount To Be Paid

$\$$

$\mathbf{s}$

\section{Method of Payment}

$\square$ Check. Make checks payable to the AMS. For all check payments, please keep a copy of this form for your records

ㅁ Credit Card. All major credit cards accepted. For your security, we do not accept credit card numbers by email, fax, or postal mail. If the MMSB receives your registration form by any of these methods, it will contact you at the phone number provided on this form.

Signature:

口 Purchase Order \# (please enclose copy)

\section{Other Information}

Mathematical Reviews primary field of interest \#

$\square \quad$ I am willing to serve as a judge for the MAA Undergraduate Student Poster Session.

ㅁ If you are an undergraduate, are you interested in participating in the Radical Dash, a multi-day scavenger hunt sponsored by the MAA?

$\square$ For planning purposes for the MAA Two-year College Reception, please check if you are a faculty member at a two-year college.

$\square$ Please $\checkmark$ this box if you have a disability requiring special services.

To respect your privacy and to better serve you, please indicate your preferences for the following:

ㅁ Please include my name and affiliation on the JMM Participant List.

$\square$ Please include my name and postal address on promotional mailing lists

Registration for the Joint Meetings is not required for the short course but it is required for the minicourses and the Employment Center. To register for the Employment Center, go to www.ams.org/profession/employment-services. For questions, email emp-info@ams.org

\section{Registration Deadlines}

To be eligible for the complimentary hotel room lottery: In time to receive programs in the mail:

Oct. 30,2018

Hotel reservations with check deposit:

Nov. 20, 2018

Hotel reservations, changes/cancellation

Dec. 1, 2018

through the JMM website:

Advance registration for the Joint Meetings, short course, minicourses, and dinner tickets:

Dec. 13, 2018 Total for NAM Banquet

口. AMS Social (1/19) Regular Price \#_US\$75 Student Price \#_US\$ 35 Total for AMS Social \$

Printed Meeting Program (PLEASE CHOOSE)

ㅁ Meeting Program (pick up at mtg only) US\$ 5

口 Meeting Program mailed (U.S. residents only) USS 10

Registration must be received by Nov. 20 to be eligible for shipping

口 I do not want a printed program. Total for Meeting Program/Shipping

Total for Registrations and Events
Cancel in time to receive $50 \%$ refund on advance registration, banquets, minicourses, and short course "no refunds issued after this date.
Dec. 27, 2018

Jan. 8, 2019*
Mailing Address/Contact:

Mathematics Meetings Service Bureau (MMSB)

P. O. Box 6887

Providence, RI 02940-6887 Fax: 401-455-4004; Email: mmsb@ams.org

Telephone: $401-455-4144$ or $1-800-321-4267 \times 4144$ or $\times 4137$ 

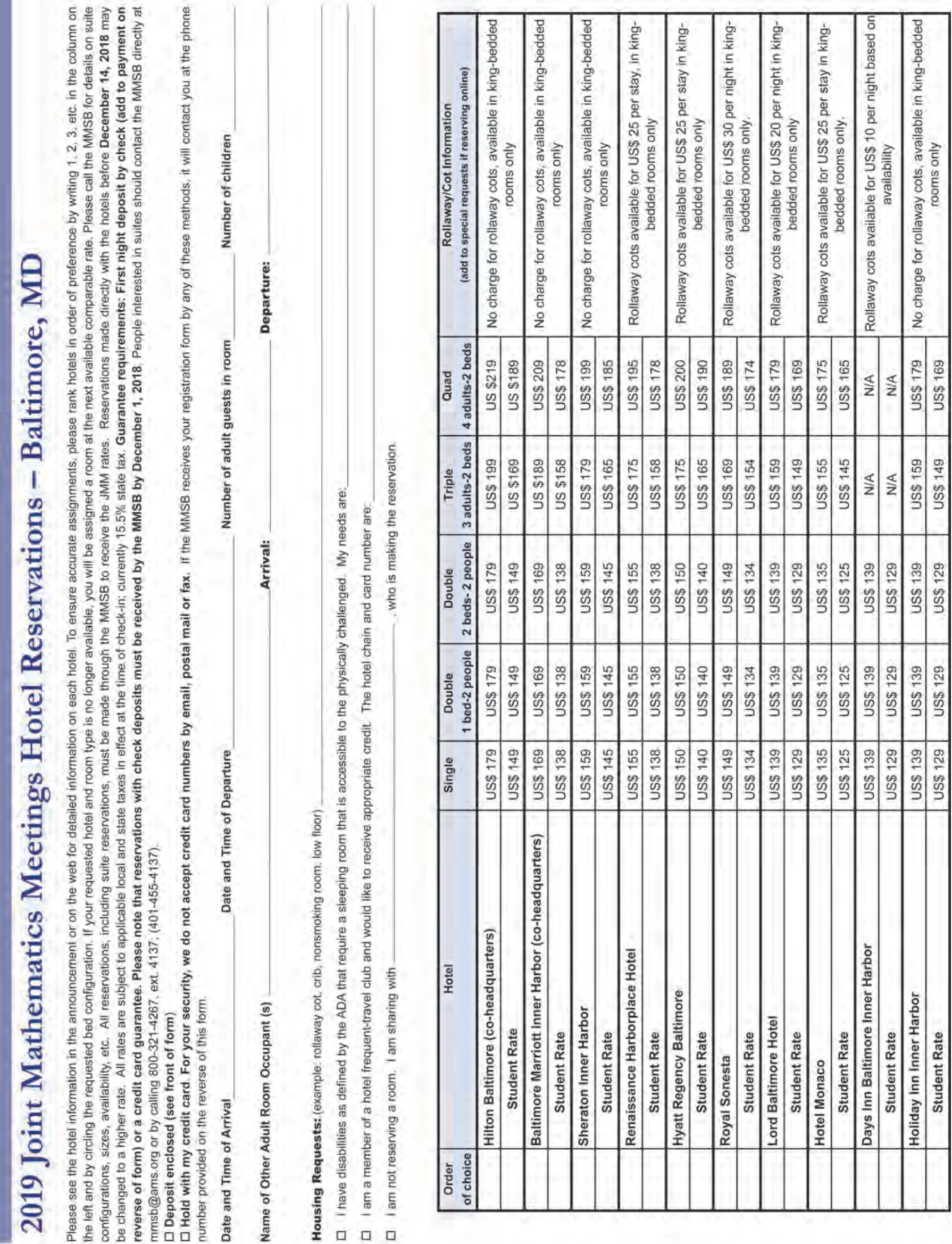

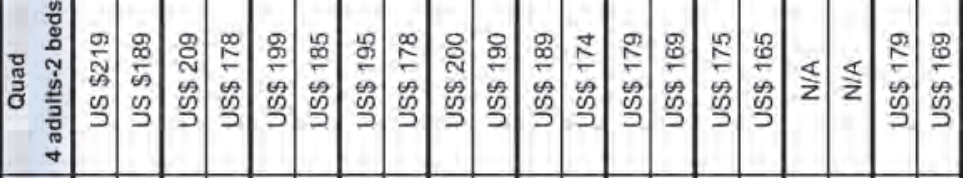

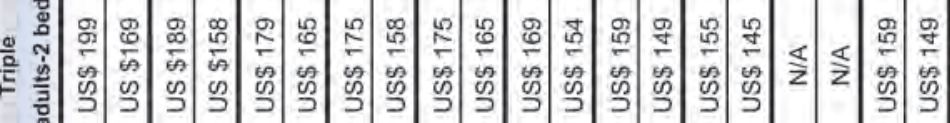

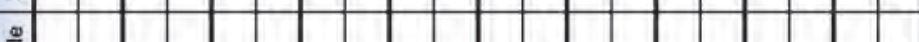

윰

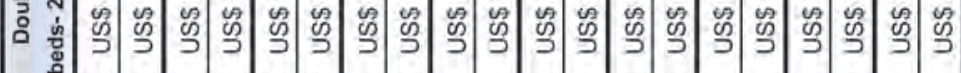

त

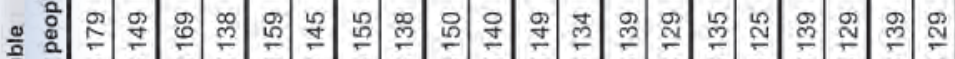

高官 की

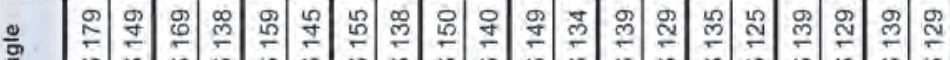

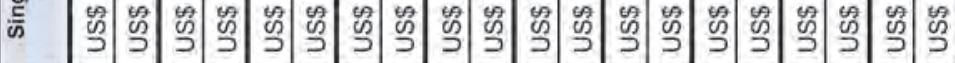

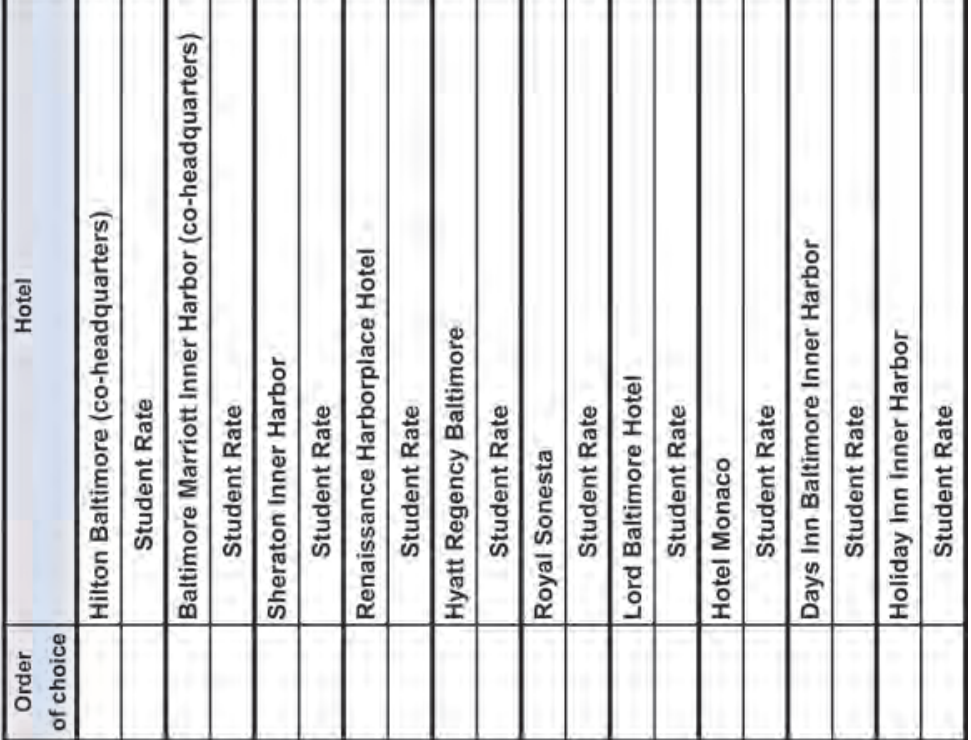




\section{IN THE NEXT ISSUE OF NOTICES}
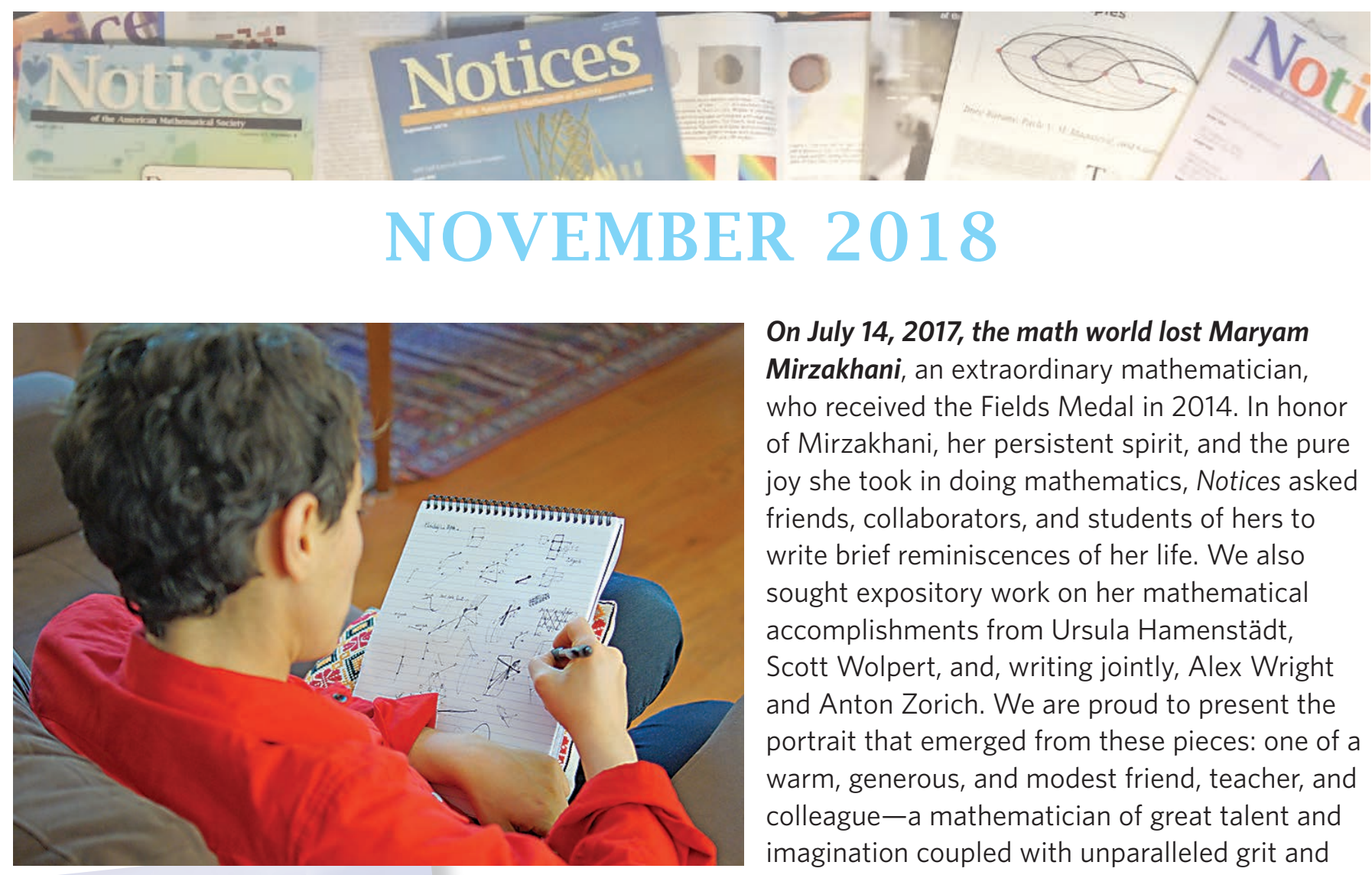

On July 14, 2017, the math world lost Maryam

Mirzakhani, an extraordinary mathematician, who received the Fields Medal in 2014. In honor of Mirzakhani, her persistent spirit, and the pure joy she took in doing mathematics, Notices asked friends, collaborators, and students of hers to write brief reminiscences of her life. We also sought expository work on her mathematical accomplishments from Ursula Hamenstädt, Scott Wolpert, and, writing jointly, Alex Wright and Anton Zorich. We are proud to present the portrait that emerged from these pieces: one of a warm, generous, and modest friend, teacher, and colleague-a mathematician of great talent and imagination coupled with unparalleled grit and persistence.

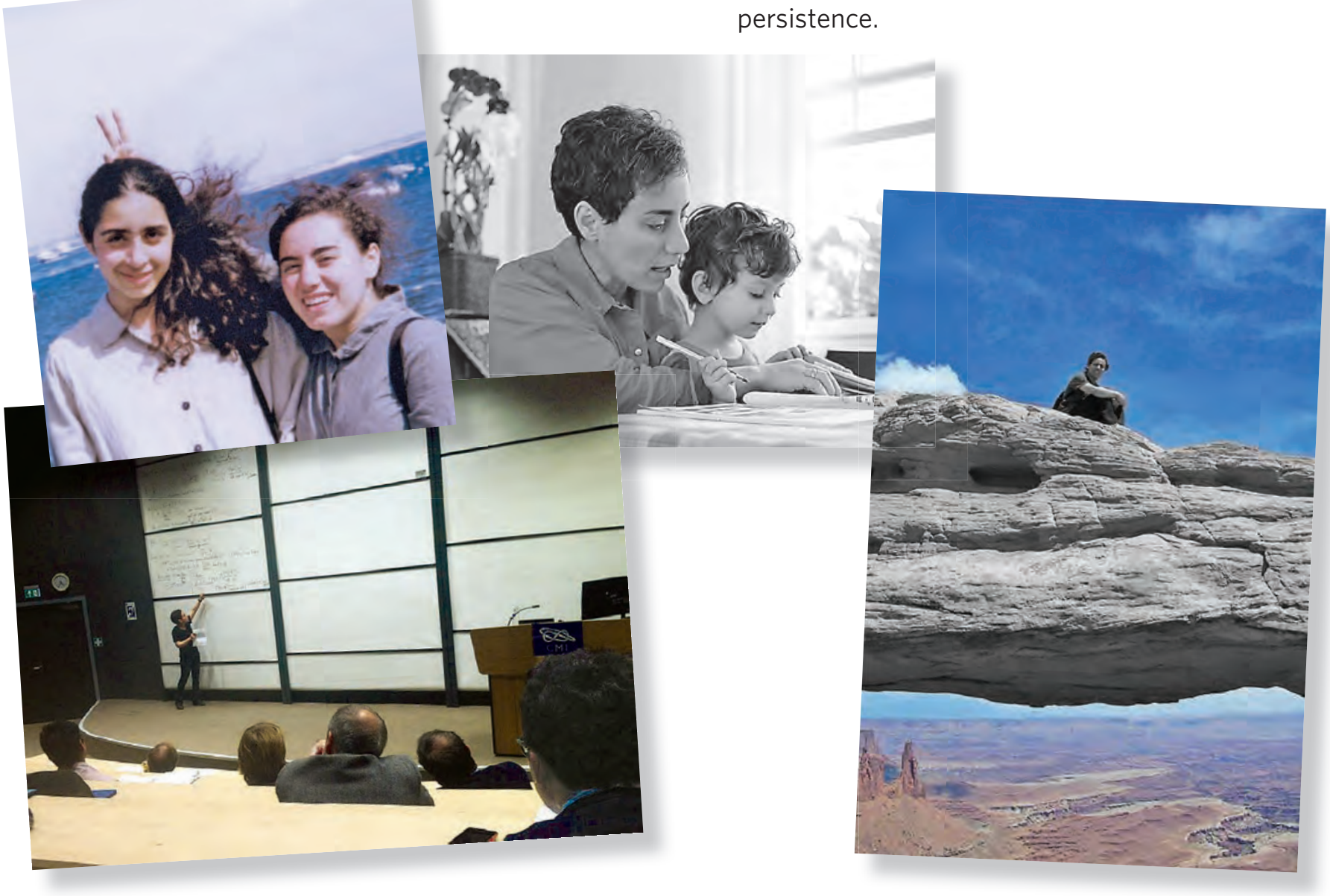




\section{American Mathematical Society}

Distribution Center

\section{Monticello Place,}

Pawtucket, RI 02861 USA

\section{NEW RELEASES from the AMS}

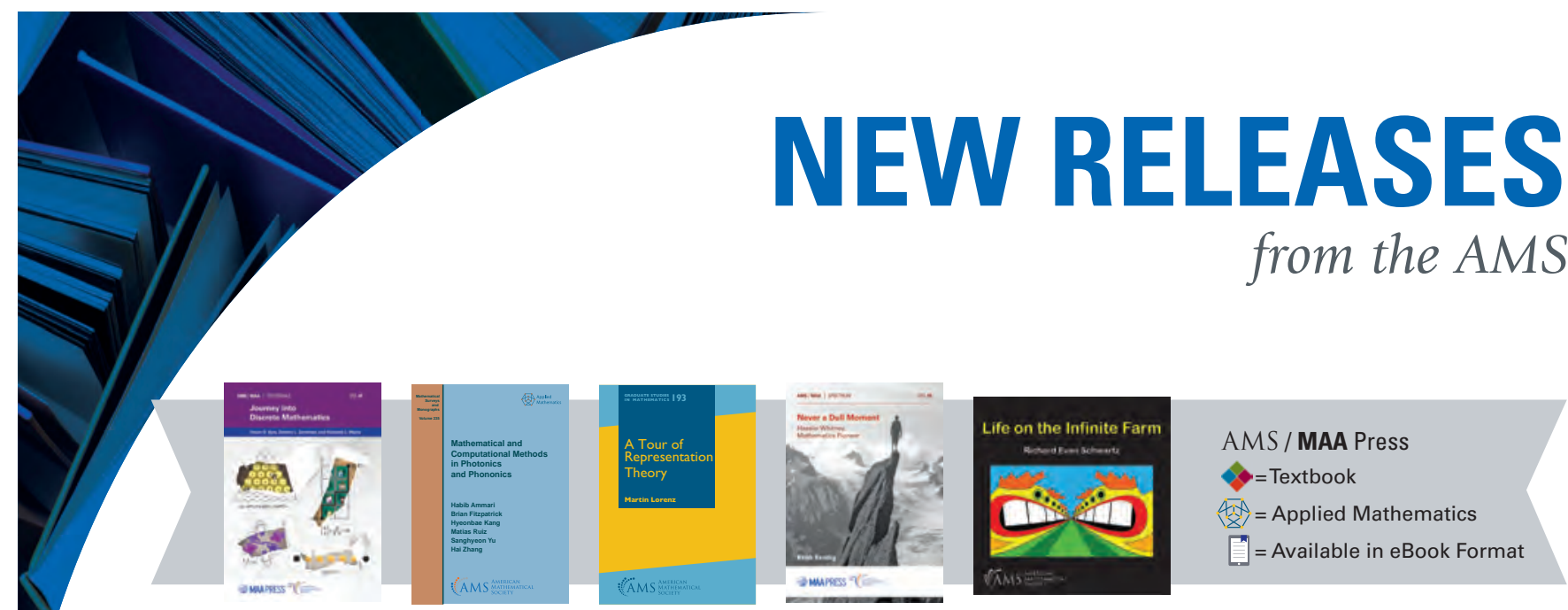

\section{Journey into Discrete Mathematics \\ AMS / MAA Press}

Owen D. Byer, Eastern Mennonite University, Harrisonburg, VA, Deirdre L. Smeltzer, Eastern Mennonite University, Harrisonburg, VA, and Kenneth L. Wantz, Regent University, Virginia Beach, VA

Journey into Discrete Mathematics promotes development of a mathematical mindset and prepares students for further study and is designed for use in a first course in mathematical abstraction for early-career undergraduate mathematics majors.

MAA Textbooks, Volume 41; 2018; approximately 389 pages; Hardcover; ISBN: 978-1-4704-4696-3; List US\$75; Individual member US\$56.25; MAA members US\$56.25; Order code TEXT/4

\section{Mathematical and Computational Methods in Photonics and Phononics}

\section{圆綮}

Habib Ammari, ETH, Zürich, Switzerland, Brian Fitzpatrick, ETH, Zürich, Switzerland, Hyeonbae Kang, Inha University, Incheon, Korea, Matias Ruiz, École Normale Supérieure, Paris, France, Sanghyeon Yu, ETH, Zürich, Switzerland, and Hai Zhang, HKUST, Clear Water Bay, Hong Kong

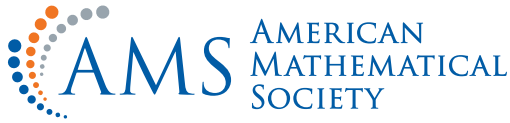

This book reviews new and fundamental mathematical tools, computational approaches, and inversion and optimal design methods to address challenging problems in photonics and phononics.

Mathematical Surveys and Monographs, Volume 235; 2018; 509 pages; Hardcover; ISBN: 978-1-4704-4800-4; List US\$135; AMS members US\$108; MAA members US\$121.50; Order code SURV/235

\section{A Tour of Representation Theory -}

Martin Lorenz, Temple University

Philadelphia, PA

Adopting a panoramic viewpoint, this book offers an introduction to four different flavors of representation theory: representations of algebras, groups, Lie algebras, and Hopf algebras.

Graduate Studies in Mathematics, Volume 193; 2018; 654 pages; Hardcover; ISBN: 978-1-4704-3680-3; List US\$94: AMS members US\$75.20; MAA members US\$84.60; Order code GSM/193

\section{Never a Dull Moment}

Hassler Whitney, Mathematics Pioneer AMS / MAA Press

Keith Kendig, Cleveland State University, $\mathrm{OH}$ This biography is a revealing portrait of a fascinating personality and a titan of twentieth-century mathematics, Hassler Whitney.
Spectrum, Volume 93; 2018; approximately 381 pages; Softcover; ISBN: 978-1-4704-4828-8; List US\$60; Individual member US\$45; MAA members US\$45; Order code SPEC/93

\section{Life on the Infinite Farm}

Richard Evan Schwartz, Brown University, Providence, RI

In Life on the Infinite Farm, mathematician and award-winning children's book author Richard Schwartz teaches about infinity and curved space through stories of whimsical farm animals.

2018; 176 pages; Softcover; ISBN: 978-1-4704-4736-6; ist US\$25: AMS members US\$20: MAA members US\$22.50; Order code MBK/115

\section{Discover more titles at}

\section{bookstore.ams.org}

